\title{
Locating median lines and hyperplanes with a restriction on the slope
}

\author{
Dissertation \\ zur Erlangung des mathematisch-naturwissenschaftlichen \\ Doktorgrades \\ "Doctor rerum naturalium" \\ der Georg-August-Universität Göttingen \\ vorgelegt von
}

Thorsten Krempasky

aus Kassel

Göttingen 2012 
Referentinnen der Dissertation:

Referentin: Prof. Dr. Anita Schöbel

Korreferentin: Asst. Prof. Ulrike Schneider

Tag der mündlichen Prüfung: 


\begin{abstract}
Hyperplane location problems as well as line location problems as a special case have been of great interest in mathematics for a long time. A lot of people have been and still are interested in the question of how to locate a hyperplane for a variety of reasons.

In this thesis, we consider the location of median lines when there is a restriction on the slope of the line to locate as well as the more general location of median hyperplanes when there is a restriction on the slope vector of the hyperplane to locate. We are given a set of existing facilities in $\mathbb{R}^{2}$ or $\mathbb{R}^{n}$ and we are searching for a new facility that is a line or a hyperplane, respectively, minimizing the sum of distances between the existing facilities and the new facility while satisfying a restriction on its slope.

We investigate this problem in the plane $\mathbb{R}^{2}$ using different distance measures, namely the vertical distance, the horizontal distance, the rectangular distance and finally distances derived from arbitrary norms. We present what kind of different geometrical features these problems have if we let the restriction vary and which features all of them share. Afterwards we generalize our results for the location of a median line using vertical distance to the location of a median hyperplane using vertical distance. We will prove that there exists an optimal trajectory that is continuous for this problem and we will present a method to construct such an optimal trajectory exploring the geometrical properties of the problem. Finally we give an idea of how to generalize the results concerning hyperplane location with vertical distance to hyperplane location problems using other distances. On our way we will point out that there is a strong connection between the location of a hyperplane with a restriction on the slope and the RLAD regression problem from the field of robust statistics.
\end{abstract}

\title{
Keywords:
}

median hyperplane location, optimal trajectory, robust regression 



\section{Acknowledgement}

There are numerous people that passed my way during the writing of this theses influencing me and my work in one way or another.

I am very grateful to my supervisor Prof. Dr. Anita Schöbel not only for her advice and for the discussions that helped me to push my work forward, but especially for making it possible to be a $\mathrm{PhD}$ student and still having enough time for my family. Furthermore, I want to thank Asst. Prof. Ulrike Schneider for taking the Koreferat and for bringing me in contact with the field of regularized regression analysis in the first place.

The writing of this theses was financially supported by the DFG Research Training Group 1023 "Identification in Mathematical Models". I am thankful for this support as well as for the flexibility this group offers to parents.

Many thanks go to my past and present colleagues of the working group "Discrete Optimization" for the nice mixture of discussion, coffee breaks and nights out that we shared. Special thanks go to Robert Schieweck for proofreading this thesis.

I cannot express how grateful I am to my friends and my family. For just being there and sharing lots of perfect moments I thank the whole Kassel Crew.

I would not be who I am today without my Mum, my Dad and my brother being on my side no matter what happens.

For putting mathematics out of my mind and for showing me what being curious really means I thank my daughter Daria. I thank Denise for believing in me and making me laugh at the end of day no matter how terrible the day has been. 



\section{Contents}

\begin{tabular}{lll}
\hline 0 & Introduction and basic concepts & 7
\end{tabular}

0.1 Introduction . . . . . . . . . . . . . . . . . . . . . . . 7

0.2 Basic concepts of line location and hyperplane location with a restriction on the slope . . . . . . . . . . . . . . 10

I Locating lines with a restriction on the slope using vertical dis\begin{tabular}{l|l} 
tance & 15
\end{tabular}

I.1 Line location with vertical distance . . . . . . . . . . . . . . . 15

I.2 Locating lines with vertical distance and a restriction on the slope . 22

II An excursion to statistics: RLAD regression and its relation to locating lines and hyperplanes

III Locating lines with a restriction on the slope using horizontal distance

III.1 Locating lines with horizontal distance and a restriction on the slope 50

III.2 The relationship between line location using vertical distance and line location using horizontal distance . . . . . . . . . . . . 70

\begin{tabular}{lll}
\hline IV Locating lines with a restriction on the slope using $l_{1}$-distance & 77
\end{tabular}

IV.1 Line location with rectangular distance . . . . . . . . . . . . . 77

IV.2 Line location with rectangular distance and a restriction on the slope 82

\begin{tabular}{|l}
$\mathrm{V}$ \\
$\begin{array}{l}\text { Locating hyperplanes with a restriction on the slope using vertical } \\
\text { distance }\end{array}$
\end{tabular}

V.1 The problem of locating hyperplanes using vertical distance. . . . . 106 


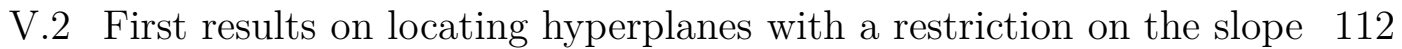

V.3 The existence of a continuous optimal trajectory . . . . . . . . . . . 119

V.4 How to construct a continuous optimal trajectory . . . . . . . . . . 147

VI Extensions

175

VI.1 Locating lines in the normed plane with a restriction on the slope . 175

VI.2 Locating hyperplanes with a restriction on the slope considering

horizontal and rectangular distance - an outlook . . . . . . . . . 184 


\section{Chapter 0}

\section{Introduction and basic concepts}

\section{$0.1 \quad$ Introduction}

Facility location has become a vast field in applied mathematics. There are a lot of problems and applications that can be tackled with locational analysis, for an overview see e.g. [DH02]. In this work we want to consider line location problems in the plane with restrictions on the slope of the line as well as hyperplane location problems with a restriction on the slope. Hyperplane location problems are a generalization of line location problems since in $\mathbb{R}^{2}$ the hyperplanes are exactly the lines. Line location as well as hyperplane location in general is in a way an extension of classical facility location.

In classical facility theory we have a set of existing facilities in the plane with nonnegative weights indicating the importance of the different facilities. We search for a location of one or more new facilities in such a way that the new facilities are as close as possible to the existing ones. Common objective functions are for example the median function or the center function. The median function is the (weighted) sum of distances between the existing facilities and the new facilities, while the center function gives the maximum (weighted) distance between the existing facilities and the new facilities. The aim is to minimize these functions. Of course there are many more objective functions possible, for example ones with negative weights or functions derived by special distance measures between the existing facilities and the new ones. Moreover, a lot of extensions of these problems 
are possible, for example by restrictions on the location of the new facilities. An overview about the topic of classical facility location in the plane is for example given in LMW88, Ham95, Pla95].

In the case of hyperplane location, we not only want to locate a single point in $\mathbb{R}^{n}$ but a hyperplane. Our aim is still the same: We want to minimize the distance between a given set of existing facilities in $\mathbb{R}^{n}$ and the new hyperplane. In the special case of $n=2$ this is the classical line location. Hyperplane location belongs to the area of locating dimensional facilities such as paths, lines, line segments, trees, circles, hyperplanes or hyperspheres. Problems of this kind have been extensively studied in networks (for an overview see [HSL93] or [MB96]), but there are also studies about the location of dimensional structures in the plane or in $\mathbb{R}^{n}$, for example [Sch99, DBMS04, BJS09, BCH09, Kör11]. Locating a hyperplane in $\mathbb{R}^{n}$ with a restriction on the slope obviously is a special case of hyperplane location in $\mathbb{R}^{n}$.

One nice thing about hyperplane location and line location in particular is that it plays an important role in a variety of different mathematical fields. We want to present a number of examples in order to give an idea in which mathematical fields such problems can arise.

One problem from the field of location theory that leads to locating a line with a restriction on the slope is the problem of locating a line segment. In [Sch99], the line segment location problem with vertical distance in the plane is described. Given a real number $l_{0}>0$ and a set $\mathcal{E} x=\left\{E x_{1}, E x_{2}, \ldots, E x_{m}\right\}$ of points in the plane representing the existing facilities, we are looking for a line segment $S$ with Euclidean length $l_{0}$ such that the sum of vertical distances (or the maximum vertical distance) between the existing facilities and the line segment is minimized. It is shown in [Sch99] that in order to solve the line segment location problem it is sufficient to solve a line location problem with vertical distance and a certain restriction on the slope of the line. We will discuss this type of problem in Chapter I.

Another field in which hyperplane location problems are of interest is com- 
putational geometry. The linear $l_{1}$ approximation problem for example seeks a vector $x \in \mathbb{R}^{n}$ to minimize the deviation between $A x$ with $A \in \mathbb{R}^{m \times n}$ and $b \in \mathbb{R}^{m}$ in the sense of the $l_{1}$-norm, i.e. the objective function is the sum of the absolute values of the deviations. This is indeed a hyperplane location problem. If $n=2$, we have again a line location problem. This kind of problem is for example studied in [BR73] or [KM93].

Line location problems as well as hyperplane location problems in general are well-known in the field of robust statistics. They play an important role in the field of regression analysis. Finding a regression line corresponds to a line location problem using some special function to measure the distance between a set of given data points and the regression line. Since statisticians usually deal with a large amount of data, they are especially interested in finding regression hyperplanes in $\mathbb{R}^{n}$ which again corresponds to a hyperplane location problem with the appropriate function to measure the distance between data points in $\mathbb{R}^{n}$ and the regression hyperplane. Problems of this kind are for example investigated in [Ric64, Sha71, PFTV86, Edg88, GP02]. A more recent topic in robust statistics is regularized regression analysis. The objective functions in this field are of the form "loss + penalty", where the "loss" part is again some special function to measure the distance between the given set of data points and the regression hyperplane, while the "penalty" part is usually the $l_{1}$-norm of the slope of the regression hyperplane.

The problem of finding a regularized regression hyperplane corresponds to the problem of locating a hyperplane using some special distance with a restriction on the slope of the hyperplane. This is why we will point out the connection between our problem and a special problem in the field of regularized regression - namely the so-called RLAD regression - in detail in Chapter 【I. We will discuss this connection for the special case of a regression line as well as for the general case of a regression hyperplane. Surveys about this type of problems can be found in [Tib96, EHJT04, WGZ06, RZ07].

As one can see, hyperplane location problems as well as the special case of line location problems are of interest in various fields of mathematics. In this work, we 


\subsection{BASIC CONCEPTS OF LINE LOCATION AND HYPERPLANE LOCATION WITH A RESTRICTION ON THE SLOPE} 10

will mainly focus on line location problems with a restriction on the slope. There seems to be known very little about these problems in the literature, this is why we want to discuss them in this work. In Chapter I, Chapter III, Chapter IV] and in parts of Chapter VI we will discuss line locating problems with a restriction on the slope considering different distances. In Chapter $\mathrm{V}$ and parts of Chapter VI we discuss the general case of locating a hyperplane in $\mathbb{R}^{n}$ with a restriction on the slope considering different distances.

\subsection{Basic concepts of line location and hyper- plane location with a restriction on the slope}

Since the main part of this work addresses the location of a line with a restriction on the slope, we want to start with the description of the basic concept of such a problem. In order to do so, let $\mathcal{E}=\left\{\left(x_{1}, y_{1}\right), \ldots,\left(x_{n}, y_{n}\right)\right\} \subseteq \mathbb{R}^{2}$ be a set of existing facilities in the plane with weights $w_{i} \in \mathbb{R}_{+}$and let $d$ be a distance function on the plane, i.e. $d\left(\left(x_{i}, y_{i}\right),\left(x_{j}, y_{j}\right)\right)$ defines a distance between facility $\left(x_{i}, y_{i}\right)$ and facility $\left(x_{j}, y_{j}\right)$. The weights might indicate the importance of the different facilities. There are many possibilities to choose a distance function, the Euclidean distance or the rectangular distance are for example common distance functions. We now search for a location of a new facility $(x, y) \in \mathbb{R}^{2}$ that minimizes the weighted sum of distances defined by $d$ between the existing facilities and the new facility $(x, y)$, which means that we have to solve an optimization problem of the following form:

$$
\min \sum_{i=1}^{n} w_{i} d\left((x, y),\left(x_{i}, y_{i}\right)\right) \text {. }
$$

In our special case, the new facility that has to be located is a line $l_{a, b}$ with slope $a \in \mathbb{R} \cup\{\infty\}$ and intercept $b \in \mathbb{R}$ and we will let $w_{i}=1$ for every existing facility $\left(x_{i}, y_{i}\right) \in \mathcal{E}$. Here, $a \in \mathbb{R} \cup\{\infty\}$ means that the new line can be a non-vertical line with slope $a \in \mathbb{R}$ and intercept $b \in \mathbb{R}$, that is 


$$
l_{a, b}=\left\{(x, y) \in \mathbb{R}^{2}: y=a x+b\right\},
$$

or a vertical line with infinite slope passing the point $(b, 0)$, that is

$$
l_{\infty, b}=\left\{(x, y) \in \mathbb{R}^{2}: x=b\right\}
$$

We will see in Chapter I that we only have to investigate non-vertical lines $l_{a, b}$ with $a, b \in \mathbb{R}$ when we talk about the vertical distance.

We can now formulate the optimization problem of locating a line:

$$
\min \sum_{i=1}^{n} d\left(\left(x_{i}, y_{i}\right), l_{a, b}\right)
$$

This function is called unweighted median function, this is why we will call an optimal line of this problem a median line as well. The distance $d\left(\left(x_{i}, y_{i}\right), l_{a, b}\right)$ between an existing facility $\left(x_{i}, y_{i}\right) \in \mathcal{E}$ and a line $l_{a, b}$ is given by the distance between $\left(x_{i}, y_{i}\right)$ and its nearest point on the line, that is to say

$$
d\left(\left(x_{i}, y_{i}\right), l_{a, b}\right)=\min _{(x, y) \in l} d\left(\left(x_{i}, y_{i}\right),(x, y)\right)
$$

Up to now, $\left(P_{d}\right)$ is a classical line location problem. We will now introduce a restriction on the slope of the line:

$$
\begin{aligned}
& \min \sum_{i=1}^{n} d\left(\left(x_{i}, y_{i}\right), l_{a, b}\right) \\
& \text { s.t. }|a| \leq s
\end{aligned}
$$

where $s \geq 0$.

This is the class of problems we have in mind when we talk about locating a line with a restriction on the slope. We will discuss how to solve $\left(P_{d}(s)\right)$ for any $s \geq 0$ using different distance measures $d$. Moreover we will see what different features $\left(P_{d}(s)\right)$ has for different distance measures. In Chapter $\llbracket$ we start with the problem 


\subsection{BASIC CONCEPTS OF LINE LOCATION AND HYPERPLANE LOCATION WITH A RESTRICTION ON THE

of locating a line with a restriction on the slope using the vertical distance. We show that there exists what is called an optimal trajectory that is continuous in $s$. Based on the results in this chapter we will investigate the problem $\left(P_{d}(s)\right)$ with $d$ being the horizontal distance in Chapter III, Bringing the results of Chapter I and Chapter III together, in Chapter IV we deal with the problem $\left(P_{d}(s)\right)$ when $d$ is the rectangular distance. Finally, we will generalize our results to the problem of line location with a distance function derived from an arbitrary norm in Chapter VI.

In Chapter $\mathrm{V}$ we will consider the problem of locating a hyperplane with a restriction on the slope using the vertical distance. Nevertheless we shortly want to describe the basic concept of locating a non-vertical hyperplane with a restriction on the slope in general since we want to discuss the possibility of extending the results derived for the vertical distance to problems with horizontal distance and rectangular distance in Chapter VI. We will introduce the vertical distance between points in the $\mathbb{R}^{n}$ as well as the vertical distance between a point and a hyperplane in Section V.1.

In this work we only want to investigate the location of non-vertical hyperplanes. We will see in Chapter $\mathrm{V}$ and Chapter $\mathrm{VI}$ why this is sufficient for the problems that we consider.

Let $a=\left(a_{1}, \ldots, a_{k}\right) \in \mathbb{R}^{k}$ and $b \in \mathbb{R}$. Any non-vertical hyperplane $H$ can be expressed as $H_{a, b}$ with

$$
H_{a_{1}, \ldots, a_{k}, b}=\left\{\left(x_{1}, \ldots, x_{k}, y\right) \in \mathbb{R}^{k+1}: y=a_{1} x_{1}+a_{2} x_{2}+\ldots+a_{k} x_{k}+b\right\} .
$$

As we already mentioned, the problem of locating a hyperplane with a restriction on the slope is of course a generalization of the line location problem with a restriction on the slope $\left(P_{d}(s)\right)$. Therefore we want to use a notation very similar to the one we use for $\left(P_{d}(s)\right)$. Let

$$
\mathcal{E}=\left\{\left(x_{11}, x_{12}, \ldots, x_{1 k}, y_{1}\right), \ldots,\left(x_{n 1}, x_{n 2}, \ldots, x_{n k}, y_{k}\right)\right\} \subseteq \mathbb{R}^{k+1}
$$

be the set of existing facilities in the $\mathbb{R}^{k+1}$. Again we let $d$ be a distance function defined on $\mathbb{R}^{k+1}$ that defines a distance between two points in the $\mathbb{R}^{k+1}$. We search 
for the location of a new facility that is a non-vertical hyperplane $H_{a_{1}, \ldots, a_{k}, b}$ such that the sum of distances defined by $d$ between the existing facilities in $\mathcal{E}$ and $H_{a_{1}, \ldots, a_{k}, b}$ is minimized. In other words we want to solve the following optimization problem:

$$
\begin{aligned}
& \min \sum_{i=1}^{n} d\left(\left(x_{i 1}, \ldots, x_{i k}, y_{i}\right), H_{a_{1}, \ldots, a_{k}, b}\right) \\
& \text { s.t. }\|a\|_{1}=\left|a_{1}\right|+\ldots+\left|a_{k}\right| \leq s
\end{aligned}
$$

where $s \geq 0$. As in the case of line location, the distance $d\left(\left(x_{i 1}, \ldots, x_{i k}, y_{i}\right), H\right)$ between an existing facility $\left(x_{i 1}, \ldots, x_{i k}, y_{i}\right) \in \mathcal{E}$ and a hyperplane $H_{a_{1}, \ldots, a_{k}, b}$ is given by the distance between $\left(x_{i 1}, \ldots, x_{i k}, y_{i}\right)$ and its nearest point on the hyperplane:

$$
\begin{aligned}
& d\left(\left(x_{i 1}, \ldots, x_{i k}, y_{i}\right), H_{a_{1}, \ldots, a_{k}, b}\right) \\
= & \min _{\left(x_{1}, \ldots, x_{k}, y\right) \in H_{a_{1}, \ldots, a_{k}, b}} d\left(\left(x_{i 1}, \ldots, x_{i k}, y_{i}\right),\left(x_{1}, \ldots, x_{k}, y\right)\right) .
\end{aligned}
$$

What we are going to show in Chapter $\bar{V}$ is that there exists an optimal trajectory for the problem with vertical distance $\left(P_{v e r}(s)\right)$ that is continuous in $s$. This is the generalisation of the statement about the existence of a continuous optimal trajectory for the line location problem with vertical distance we made in Chapter [1] to the case of hyperplane location.

In Chapter VI we will discuss if the results derived in Chapter V can be carried over to the corresponding hyperplane location problems with horizontal distance and rectangular distance. 



\section{Chapter I}

\section{Locating lines with a restriction on the slope using vertical distance}

In this chapter we want to consider the problem $\left(P_{d}(s)\right)$ if the distance $d$ is the vertical distance. We will provide the definition of the vertical distance between two points as well as the vertical distance between a point and a line and start with a summary of important results for the line location problem with vertical distance. Afterwards we give a more formal description of our problem with a restriction on the slope and develop a solution path continuous in $s$ providing an optimal solution for any $s$, a so called continuous optimal trajectory.

\section{I.1 Line location with vertical distance}

In this section we briefly want to summarize some important results concerning line location with vertical distance. Our main focus will lie on the work of [Sch99].

First of all, to understand line location with vertical distance one has to know what we have in mind when talking about vertical distance. For this reason we give a definition of the vertical distance between two points in the plane.

Definition I.1.1. Let $\left(x_{1}, y_{1}\right),\left(x_{2}, y_{2}\right) \in \mathbb{R}^{2}$. The vertical distance between $\left(x_{1}, y_{1}\right)$ 
and $\left(x_{2}, y_{2}\right)$ is defined as

$$
d_{v e r}(x, y)=\left\{\begin{array}{l}
\left|y_{2}-y_{1}\right| \text { if } x_{1}=x_{2} \\
\infty \text { else. }
\end{array}\right.
$$

This distance does not seem to be very useful at a first glance, since the vertical distance between most of the points in $\mathbb{R}^{2}$ will be equal to $\infty$. On the other hand, if we look at the distance between a point and a non-vertical line, we will generally get a vertical distance which is finite. Following the idea of the distance between a single point and a set of points, the vertical distance between a point $x=\left(x_{1}, y_{1}\right) \in \mathbb{R}^{2}$ and a non-vertical line

$$
l=l_{a, b}=\left\{\left(z_{1}, z_{2}\right) \in \mathbb{R}^{2}: z_{2}=a z_{1}+b\right\}
$$

is given by the length of the vertical segment between $x$ and $l$ :

$$
\begin{aligned}
d_{v e r}(x, l) & =\min _{\left(z_{1}, z_{2}\right) \in l} d_{v e r}\left(\left(x_{1}, y_{1}\right),\left(z_{1}, z_{2}\right)\right) \\
& =d_{v e r}\left(\left(x_{1}, y_{1}\right),\left(z_{1}, z_{2}\right)\right) \text { with }\left(z_{1}, z_{2}\right)=\left(x_{1}, a x_{1}+b\right) \\
& =\left|y_{1}-\left(a x_{1}+b\right)\right|
\end{aligned}
$$

In the special case that $l$ is a vertical line and $x \notin l$ the vertical distance between $x$ and $l$ is $d_{\text {ver }}(x, l)=\infty$, whereas $d_{\text {ver }}(x, l)=0$ for all $\left(z_{1}, z_{2}\right) \in l$.

Assume that a set of existing facilities $\mathcal{E}=\left\{\left(x_{1}, y_{1}\right), \ldots,\left(x_{n}, y_{n}\right)\right\}$ is given. We want to locate a non-vertical line $l_{a, b}$ in such a way that the sum of vertical distances between the existing facilities and the line is minimized. We call this the line location problem with vertical distance and our objective function is of the form 


$$
\begin{aligned}
\min \|y-(a x+b e)\|_{1} & =\min \sum_{i=1}^{n}\left|y_{i}-\left(a x_{i}+b\right)\right| \\
& =\min \sum_{i=1}^{n} d_{v e r}\left(\left(x_{i}, y_{i}\right), l_{a, b}\right) \\
& =f_{v e r}(a, b)
\end{aligned}
$$

with $e=(1, \ldots, 1)^{t} \in \mathbb{R}^{n}$.

Note that a vertical line can never be optimal for $\left(P_{v e r}\right)$ unless all existing facilities are lying on a vertical line, in other words all existing facilities share the same first coordinate $x_{i}=x$ for all $i \in\{1, \ldots, n\}$. In this special case, the vertical line passing through the point $(x, 0)$ is optimal for $\left(P_{v e r}\right)$. Since this case is trivial we will neglect it in the following, which is why we only have to investigate non-vertical lines $l_{a, b}$ with $a, b \in \mathbb{R}$ for the rest of this chapter.

One way to cope with $\left(P_{v e r}\right)$ is to use methods of classical facility location. Another one is to use geometric properties of the line location problem. In [Sch99], both approaches are used to discuss such problems. We want to focus mainly on the use of geometric properties.

In order to take advantage of the geometric properties of $\left(P_{v e r}\right)$, we will now introduce a dual interpretation of line location problems with vertical distance like it is done in [Sch99].

The following transformation $T$ which maps points to non-vertical lines and vice versa will be crucial for our dual interpretation.

Definition I.1.2. We define the following transformation $T$ :

For any point $(x, y) \in \mathbb{R}^{2}$ let $T(x, y)$ define a non-vertical line

$$
T(x, y):=l_{-x, y}=\{(a, b): b=-x a+y\}
$$

and for any non-vertical line $l_{a, b}$ let $T\left(l_{a, b}\right)$ define a point

$$
T\left(l_{a, b}\right):=(a, b)
$$


The space of all transformed points and lines will be called the dual space.

The essential feature of the transformation $T$ defined as above is that vertical distances between straight lines and points are invariant under $T$, as the following lemma will show.

Lemma I.1.1. ([Sch99]) Let $(x, y) \in \mathbb{R}^{2}$ be a point and l a line. Then

$$
d_{v e r}((x, y), l)=d_{v e r}(T(l), T(x, y))
$$

In particular it holds that $(x, y) \in l \Leftrightarrow T(l) \in T(x, y)$.

Proof. See [Sch99].

Using this lemma, the following theorem can be proven.

Theorem I.1.1. ([Sch99]) The location of a line minimizing the sum of vertical distances to a given set of points $\left\{\left(x_{1}, y_{1}\right), \ldots,\left(x_{n}, y_{n}\right)\right\}$ is equivalent to the location of a point minimizing the sum of vertical distances to a given set of lines $\left\{T\left(x_{1}, y_{1}\right), \ldots, T\left(x_{n}, y_{n}\right)\right\}$.

Proof. Nothing left to prove after Lemma I.1.1.

In the dual space we are therefore searching for a point $(a, b)$ that minimizes the sum of vertical distances to a given set of straight lines. For a point $(a, b)$ in the dual space our objective function $f_{\text {ver }}$ can be rewritten as

$$
\begin{aligned}
f_{v e r}(a, b) & =\sum_{i=1}^{n} d_{v e r}\left(\left(x_{i}, y_{i}\right), l_{a, b}\right) \\
& =\sum_{i=1}^{n} d_{v e r}\left((a, b), l_{-x_{i}, y_{i}}\right)
\end{aligned}
$$

This tells us that a point $(a, b)$ minimizes the "dual" objective function if and only if $l_{a, b}$ is an optimal line for the line location problem with vertical distance in our initial space. 


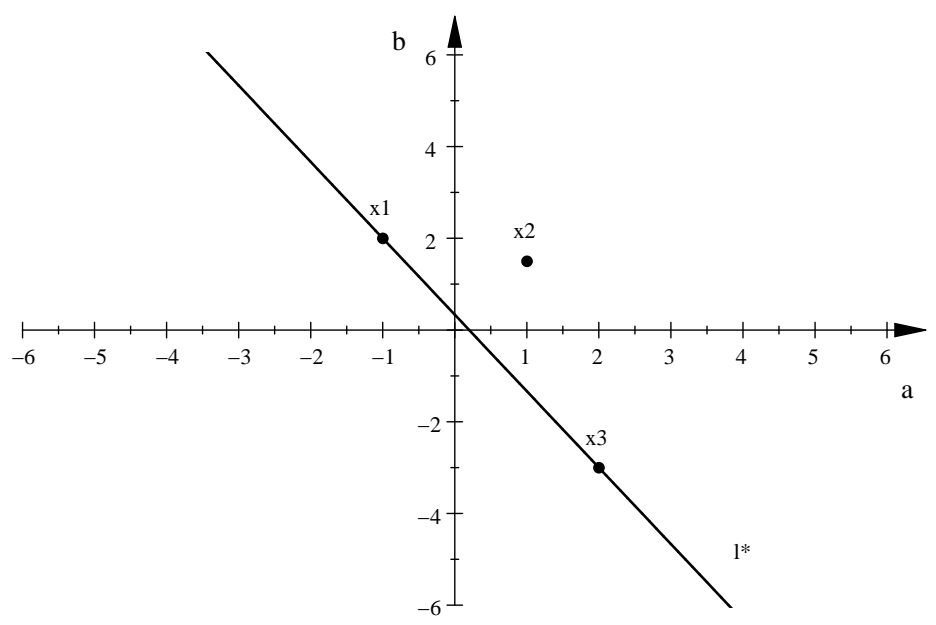

Figure I.1.1. An example with three existing facilities and an optimal solution $l^{*}$ for the unrestricted problem $\left(P_{v e r}\right)$.

Using the dual interpretation of the line location problem with vertical distance, we can state a candidate set for an optimal solution to the problem. Let

$$
\mathcal{L}=\left\{l_{-x_{i}, y_{i}}=T\left(x_{i}, y_{i}\right): i=1, \ldots, n\right\}
$$

be the set of straight lines given by the set of facilities $\left\{\left(x_{1}, y_{1}\right), \ldots,\left(x_{n}, y_{n}\right)\right\}$ and the transformation $T$. This set of lines partitions the dual $\mathbb{R}^{2}$ into cells $C \in \mathcal{C}$. In each cell, the sign of $\left(y_{i}-a x_{i}-b\right)$ does not change for all $i=1, \ldots n$, meaning that the objective function $f_{v e r}=\sum_{i=1}^{n}\left|y_{i}-\left(a x_{i}+b\right)\right|$ is linear on each cell. Since $f_{v e r}$ is also a convex function, our problem $\left(P_{v e r}\right)$ is a piecewise linear convex problem.

Since $f$ is linear on each cell, there exists an optimal solution $\left(a^{*}, b^{*}\right)$ of $\left(P_{v e r}\right)$ which is a vertex of a cell (see for example Theorem 1.3, [Sch99]). This is due to the wellknown fundamental theorem of linear programming (see e.g. [NW88]). Because all vertices of a cell are an intersection of at least two lines $l_{-x_{k}, y_{k}}, l_{-x_{l}, y_{l}} \in \mathcal{L}$, the optimal line $l_{a^{*}, b^{*}}$ must pass through at least two of the given facilities in the primal space, namely $\left(x_{k}, y_{k}\right)$ and $\left(x_{l}, y_{l}\right)$ due to Lemma [.1.1. This means that there always exists an optimal line for the line location problem with vertical distance which passes through at least two of the existing facilities.

Example I.1.1. Consider the given set of three existing facilities $\mathcal{E}=\left\{\left(x_{1}, y_{1}\right)\right.$, 


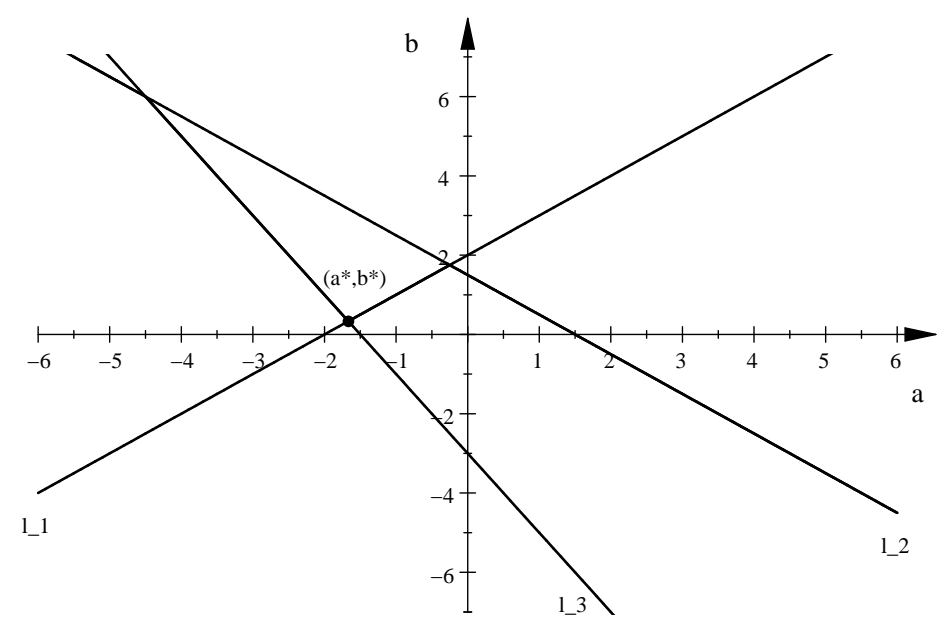

Figure I.1.2. Transformed existing facilities and optimal solution $\left(a^{*}, b^{*}\right)$ for $\left(P_{v e r}\right)$ in the dual space.

$\left.\left(x_{2}, y_{2}\right),\left(x_{3}, y_{3}\right)\right\}$ with

$$
\begin{aligned}
& \left(x_{1}, y_{1}\right)=(-1,2)=: x_{1}, \\
& \left(x_{2}, y_{2}\right)=\left(1, \frac{3}{2}\right):=x_{2} \text { and } \\
& \left(x_{3}, y_{3}\right)=(2,-3)=: x_{3} .
\end{aligned}
$$

Figure I.1.1 shows the set of existing facilities as well as an optimal solution $l^{*}$ for the unrestricted vertical problem $\left(P_{\text {ver }}\right)$. The dual space including the transformed existing facilities $l_{i}=T\left(x_{i}\right), i=1,2,3$ and the transformed optimal solution $\left(a^{*}, b^{*}\right)=T\left(l^{*}\right)$ is shown in Figure I.1.2.

Example I.1.2. Consider the given set of four existing facilities $\mathcal{E}=\left\{\left(x_{1}, y_{1}\right), \ldots\right.$, $\left.\left(x_{4}, y_{4}\right)\right\}$ with

$$
\begin{aligned}
& \left(x_{1}, y_{1}\right)=(-1,0)=: x_{1}, \\
& \left(x_{2}, y_{2}\right)=(1,2)=: x_{2}, \\
& \left(x_{3}, y_{3}\right)=(2,-1)=: x_{3} \text { and } \\
& \left(x_{4}, y_{4}\right)=(3,1)=: x_{4} .
\end{aligned}
$$


Figure 1.1 .3 shows the set of existing facilities as well as an optimal solution $l^{*}$ for the unrestricted vertical problem $\left(P_{\text {ver }}\right)$. The dual space including the transformed existing facilities $l_{i}=T\left(x_{i}\right), i=1, \ldots, 4$ and the transformed optimal solution $\left(a^{*}, b^{*}\right)=T\left(l^{*}\right)$ is shown in Figure I.1.4.

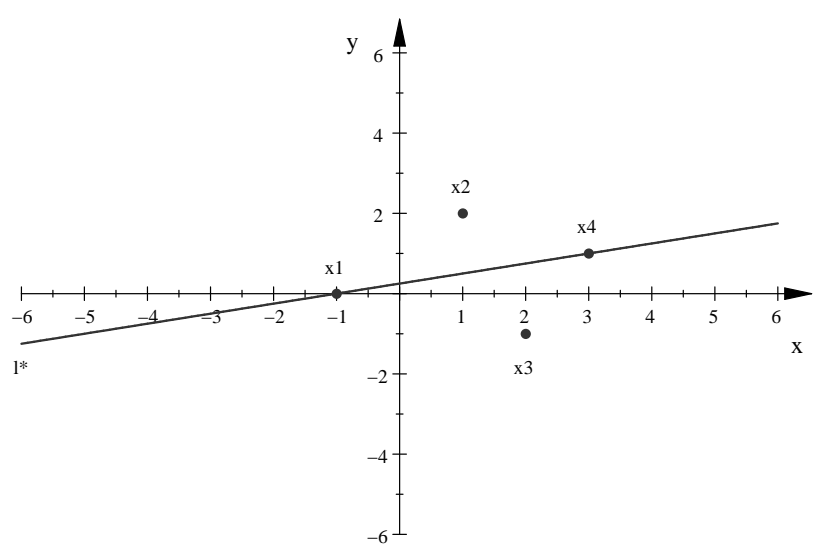

Figure I.1.3. An example with four existing facilities and an optimal solution $l^{*}$ for the unrestricted problem $\left(P_{v e r}\right)$.

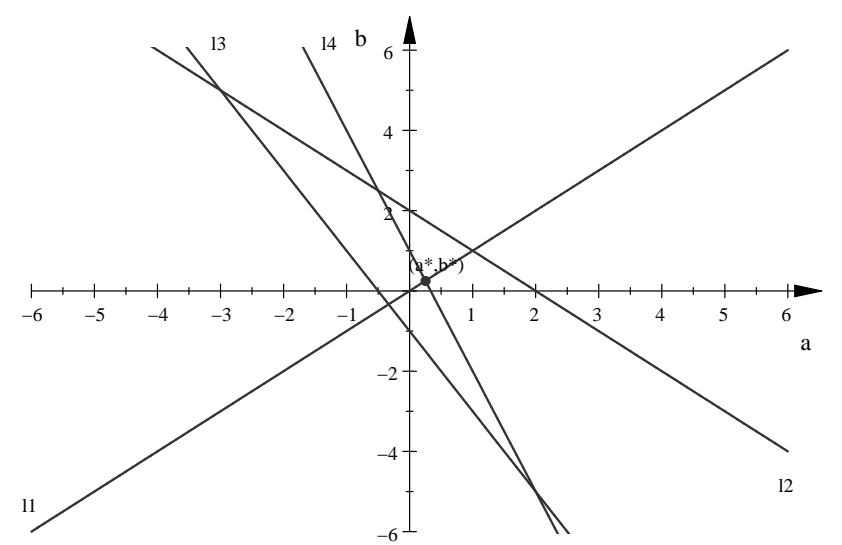

Figure I.1.4. Transformed existing facilities and optimal solution $\left(a^{*}, b^{*}\right)$ for $\left(P_{v e r}\right)$ in the dual space. 


\section{I.2 Locating lines with vertical distance and a restriction on the slope}

In the previous section, we summarized how to find a candidate set for $\left(P_{v e r}\right)$ by using the geometric properties of the problem. However, in this text we are interested in the problem of locating a line when we have a restriction on the slope of our line. In this section we want to use the results of Section I.1 in order to find a candidate set for our problem as well. In fact, we will present more than just a candidate set. We will demonstrate how to find a solution to our problem independent of the restriction we put on the slope.

Before we start we want to recall the formulation of our problem for a given $s \geq 0$ :

$$
\begin{aligned}
& \min f_{v e r}(a, b)=\min \sum_{i=1}^{n}\left|y_{i}-\left(x_{i} a+b\right)\right| \quad \quad\left(P_{v e r}(s)\right) \\
& \text { s.t. }|a| \leq s
\end{aligned}
$$

This problem corresponds to a line location problem with vertical distance with a constraint. Therefore we are going to use the dual space introduced in the previous section in order to solve $\left(P_{v e r}(s)\right)$ for any $s \geq 0$. Again we have a set of existing facilities $\mathcal{E}=\left\{\left(x_{1}, y_{1}\right), \ldots,\left(x_{n}, y_{n}\right)\right\}$ and we want to locate a line $l_{a, b}$ which minimizes the sum of vertical distances to the facilities. But this time the slope $a$ of the line must satisfy $-s \leq a \leq s$ for a positive real number $s$.

In the dual space this corresponds to the following problem: Given a set of lines $\mathcal{L}=\left\{l_{-x_{1}, y_{1}}, \ldots, l_{-x_{n}, y_{n}}\right\}$ we want to locate a point $(a, b)$ in such a way that $a$ satisfies $|a| \leq s$.

This additional constraint corresponds to a slight change in the cell structure of the dual space: In addition to the given lines in $\mathcal{L}$ we have to consider the two vertical lines passing the $a$-axis at $s$ and $-s$, respectively. In the case of a vertical line we talk of an infinite slope, therefore we denote these additional lines by $l_{\infty, s}$ and $l_{\infty,-s}$. Regarding the new cell structure, some of our former cells are split into two new cells. A solution feasible to $P_{v e r}(s)$ has to be a point in a cell which lies between $l_{\infty, s}$ and $l_{\infty,-s}$. In other words we get a subpartition of the partition of 


\section{LOCATING LINES WITH A RESTRICTION ON THE SLOPE USING VERTICAL DISTANCE}

the dual $\mathbb{R}^{2}$ induced by the lines in $\mathcal{L}$.

Therefore the sign of $y_{i}-\left(x_{i} a+b\right)$ still does not change in each cell for all $i$ which means that our problem is still piecewise linear and convex. As a consequence, there exists again an optimal solution $\left(a^{*}, b^{*}\right)$ which is a vertex of a cell. However, we have to be careful since such a vertex of a cell does not necessarily need to be an intersection of at least two lines corresponding to given facilities. It can rather be an intersection of a single line $l_{-x_{i}, y_{i}}$ corresponding to a given facility and one of the lines $l_{\infty, s}$ or $l_{\infty,-s}$ corresponding to the restriction of the slope.

This means that there need not exist an optimal line in the initial space passing through at least two of the existing facilities, but there always exists an optimal line passing through at least one of the existing facilities. We summarize this result in a theorem.

Theorem I.2.1. There exists a line optimizing $\left(P_{\text {ver }}(s)\right)$ that passes through at least one of the given facilities.

Proof. Following our explanations above there is nothing left to prove.

This observation yields a candidate set for our problem $\left(P_{v e r}(s)\right)$ : Any vertex of a cell satisfying $|a| \leq s$ is a candidate for an optimal solution. The vertices satisfying $|a|>s$ need not to be considered since they are infeasible for $\left(P_{v e r}(s)\right)$. In other words, any line either passing through at least two existing facilities or passing through at least one existing facility and having slope $s$ or $-s$ is a candidate for an optimal line.

Example I.2.1. Consider the same set of existing facilities as in Example I.1.1. Figure I.2.1 shows the dual space of the restricted problem $\left(P_{\text {ver }}(s)\right)$ with $s=$ 1 including the transformed existing facilities $l_{i}=T\left(x_{i}\right), i=1,2,3$, the two additional vertical lines corresponding to the restriction on the slope and an optimal solution $\left(a^{*}(1), b^{*}(1)\right)$ to $\left(P_{v e r}(1)\right)$.

Example I.2.2. Now consider the same set of existing facilities as in Example I.1.2. Figure I.2.2 shows the dual space of the restricted problem $\left(P_{v e r}(s)\right)$ with $s=\frac{1}{2}$ including the transformed existing facilities $L_{i}=T\left(x_{i}\right), i=1,2,3,4$, the two additional vertical lines corresponding to the restriction on the slope and an 


\section{I.2. LOCATING LINES WITH VERTICAL DISTANCE AND A}

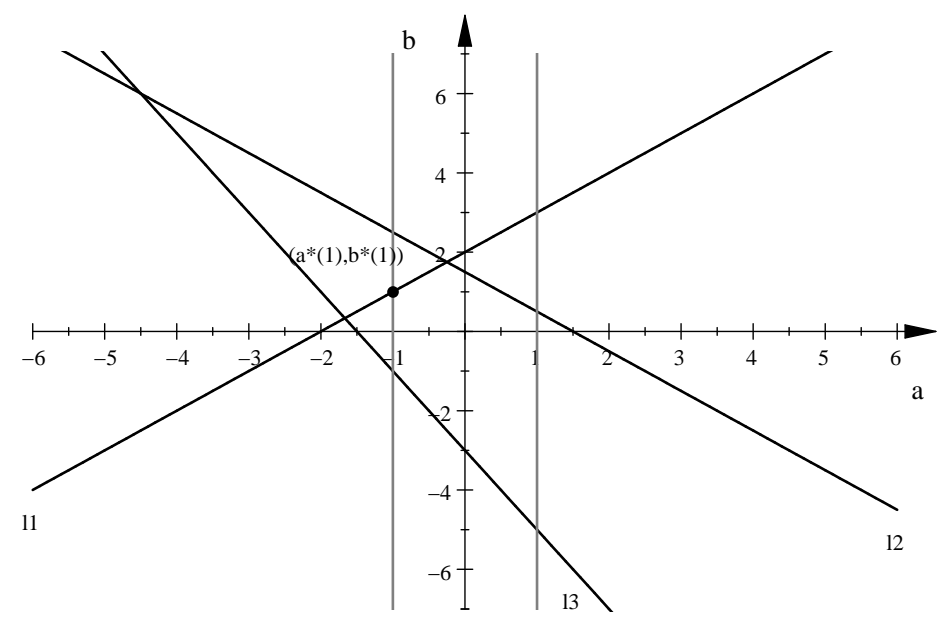

Figure I.2.1. Transformed existing facilities, additional restrictions on the slope and optimal solution $\left(a^{*}(1), b^{*}(1)\right)$ for $\left(P_{v e r}(1)\right)$ in the dual space.

optimal solution $\left(a^{*}, b^{*}\right)$ to $\left(P_{\text {ver }}\left(\frac{1}{2}\right)\right) .\left(a^{*}, b^{*}\right)$ is the same as in Example I.1.2 since the optimal solution to $\left(P_{\text {ver }}\right)$ is also feasible for $\left(P_{\text {ver }}\left(\frac{1}{2}\right)\right)$.

As we already mentioned before, we want to present a method of finding a solution to $\left(P_{v e r}(s)\right)$ for any $s$. Again we denote by $\mathcal{E}=\left\{\left(x_{1}, y_{1}\right), \ldots,\left(x_{n}, y_{n}\right)\right\}$ the set of given facilities in the plane. Let $l_{a, b}$ be a straight line with slope $a$ and intercept $b$. We distinguish between the two sets

$$
\begin{aligned}
& R\left(l_{a, b}\right)=\left\{\left(x_{i}, y_{i}\right) \in \mathcal{E}: y_{i}-\left(a x_{i}+b\right)>0\right\} \text { and } \\
& L\left(l_{a, b}\right)=\left\{\left(x_{i}, y_{i}\right) \in \mathcal{E}: y_{i}-\left(a x_{i}+b\right)<0\right\},
\end{aligned}
$$

where the set $R\left(l_{a, b}\right)$ corresponds to the set of facilities in $\mathcal{E}$ lying above the line $l_{a, b}$ while $L\left(l_{a, b}\right)$ corresponds to the set of facilities in $\mathcal{E}$ lying below this line.

In order to establish another useful feature of an optimal solution to $\left(P_{\text {ver }}(s)\right)$ we provide the definition of a pseudo-halving line which has been introduced in [Sch99] as a generalisation of the definition of a halving line. In [KM93], halving lines are introduced for Euclidean line location problems. To be more precise, in [Sch99] and [KM93] the terms of pseudo-halving hyperplanes and halving hyperplanes, respectively, are introduced for hyperplane location problems. We will use this fact in Chapter V. 


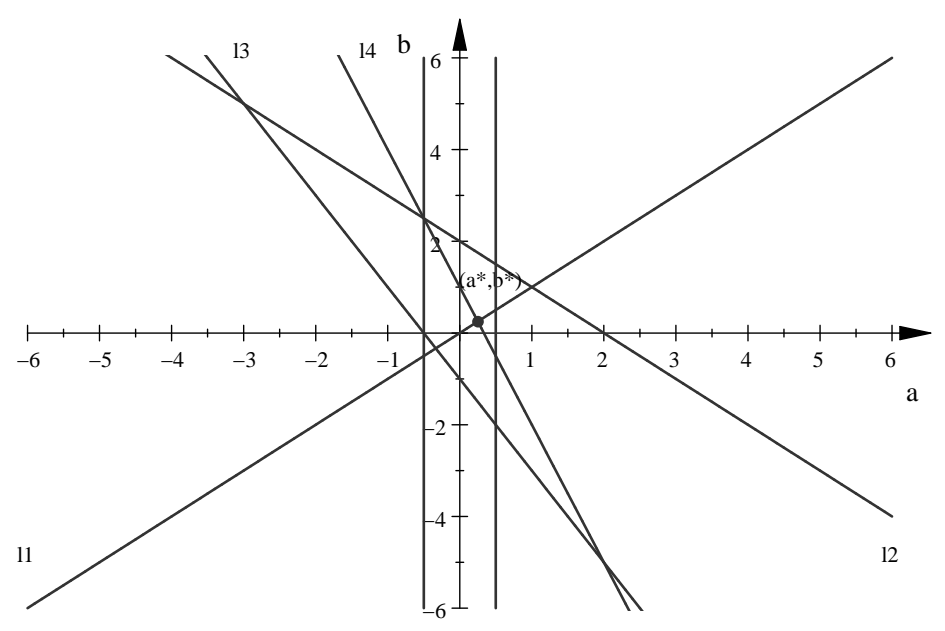

Figure I.2.2. Transformed existing facilities, additional restrictions on the slope and optimal solution $\left(a^{*}, b^{*}\right)$ for $\left(P_{v e r}\left(\frac{1}{2}\right)\right)$ in the dual space.

Let $\mathcal{E}=\left\{\left(x_{1}, y_{1}\right), \ldots,\left(x_{n}, y_{n}\right)\right\}$ be a set of given facilities in $\mathbb{R}^{2}$ with weights $w_{i}$, $W=\sum_{i=1}^{n} w_{i}$ as well as $\mathcal{B}_{l}^{+}$and $\mathcal{B}_{l}^{-}$the two open half-spaces in $\mathbb{R}^{2}$ separated by $l$.

Definition I.2.1. ([KM93]) A line $l$ which is the affine hull of two of the existing facilities and which satisfies both inequalities

$$
\sum_{\left(x_{i}, y_{i}\right) \in \mathcal{B}_{l}^{-}} w_{i}<\frac{1}{2} W
$$

and

$$
\sum_{\left(x_{i}, y_{i}\right) \in \mathcal{B}_{l}^{+}} w_{i}<\frac{1}{2} W
$$

is called halving line.

Note that the set $\left\{\left(x_{i}, y_{i}\right) \in \mathcal{B}_{l}^{-}\right\}$corresponds to the set $L(l)$ in our notation and the set $\left\{\left(x_{i}, y_{i}\right) \in \mathcal{B}_{l}^{+}\right\}$corresponds to the set $R(l)$ in our notation, respectively. KM93] showed that for the Euclidean case with $l_{2}$-norm all median lines are halving ones. Something similar is used in [MN80] for locating lines in the plane: [MN80] uses the equivalent inequation

$$
\left|\sum_{\left(x_{i}, y_{i}\right) \in \mathcal{B}_{l}^{-}} w_{i}-\sum_{\left(x_{i}, y_{i}\right) \in \mathcal{B}_{l}^{+}} w_{i}\right|<\sum_{\left(x_{i}, y_{i}\right) \in l} w_{i}
$$




\section{I.2. LOCATING LINES WITH VERTICAL DISTANCE AND A}

and shows that it holds for all median lines in the Euclidean case. Since this needs not to be true for distances derived from other norms, [Sch99] introduced the definition of a pseudo-halving line.

Definition I.2.2. ([Sch99]) A line $l$ is called pseudo-halving if

$$
\begin{gathered}
\sum_{\left(x_{i}, y_{i}\right) \in \mathcal{B}_{l}^{-}} w_{i} \leq \frac{W}{2} \text { and } \\
\sum_{\left(x_{i}, y_{i}\right) \in \mathcal{B}_{l}^{+}} w_{i} \leq \frac{W}{2} .
\end{gathered}
$$

Sticking again to our notation and in addition regarding the case with weights $w_{i}=1$ for all $i$ a line $l_{a, b}$ is pseudo-halving if

$$
\begin{aligned}
\left|L\left(l_{a, b}\right)\right| & \leq \frac{n}{2} \text { and } \\
\left|R\left(l_{a, b}\right)\right| & \leq \frac{n}{2} .
\end{aligned}
$$

[Sch99] has shown that all lines optimal for the unrestricted problem $\left(P_{v e r}\right)$ are pseudo-halving. The next theorem will show that this is true as well for any line which is optimal to $\left(P_{v e r}(s)\right)$. To prove this theorem, we first need the definition of a median to a weighted set of numbers.

Definition I.2.3. (see for example [Hay81], [Kre91], [Sch99]) Let $c_{1}, c_{2}, \ldots, c_{M}$ be a set of real numbers and let $w_{1}, w_{2}, \ldots, w_{M}$ be positive weights, $w_{m} \geq 0, m=$ $1, \ldots, M$. Let furthermore be $W=\sum_{m=1}^{M} w_{m}$ the sum of all weights. Then

$$
\operatorname{median}\left\{\left(c_{m}, w_{m}\right): m=1 \ldots M\right\}=\left\{x: \sum_{m: c_{m}<x} w_{m} \leq \frac{W}{2} \text { and } \sum_{m: c_{m}>x} w_{m} \leq \frac{W}{2}\right\}
$$

is the set of all medians to the weighted set of numbers $c_{m}$.

Now we can prove the following proposition which we again need to prove the next theorem. This proposition is a slightly different version of a statement shown for example in [Sch99], [Hav81] or [Ham95] since in our case we have a restriction on 


\section{LOCATING LINES WITH A RESTRICTION ON THE SLOPE USING VERTICAL DISTANCE}

a. Anyway, since the slope is kept fixed, this does not affect the proof. From now on we denote by

$$
\begin{aligned}
M_{v e r}^{\text {opt }}(s):= & \left\{(a, b):|a| \leq s \text { and } f_{\text {ver }}(a, b) \leq f_{\text {ver }}\left(a^{\prime}, b^{\prime}\right) \text { for all }\left(a^{\prime}, b^{\prime}\right)\right. \\
& \text { satisfying } \left.\left|a^{\prime}\right| \leq s\right\}
\end{aligned}
$$

the set of optimal solutions to $\left(P_{v e r}(s)\right)$.

Proposition I.2.1. Let $\mathcal{E}=\left\{\left(x_{i}, y_{i}\right), i=1 \ldots n\right\}$ be a set of existing facilities, $\left(a^{*}, b^{*}\right) \in M_{\text {ver }}^{\text {opt }}(s)$ and

$$
\begin{aligned}
\bar{f}: \mathbb{R} & \rightarrow \mathbb{R} \\
b & \mapsto \sum_{i=1}^{n}\left|a^{*} x_{i}-y_{i}+b\right| .
\end{aligned}
$$

$A$ value $b^{*}$ minimizes $\bar{f}$ if and only if $b^{*} \in \operatorname{median}\left\{\left(y_{i}-a^{*} x_{i}, 1\right): i=1 \ldots n\right\}$.

Proof. " $\Rightarrow$ " The function $\bar{f}$ is piecewise linear and convex.

Suppose $b^{*}$ optimizes $\bar{f}$ but $b^{*} \notin$ median $\left\{\left(y_{i}-a^{*} x_{i}, 1\right)\right\}$. Without loss of generality we can suppose that

$$
\sum_{i: y_{i}-a^{*} x_{i}<b^{*}} 1>\frac{n}{2} .
$$

Now there exists an $\epsilon>0$ so that

$$
\begin{aligned}
& \left\{i: y_{i}-a^{*} x_{i}<b^{*}\right\}=\left\{i: y_{i}-a^{*} x_{i}<b^{*}-\epsilon\right\} \text { and } \\
& \left\{i: y_{i}-a^{*} x_{i} \geq b^{*}\right\}=\left\{i: y_{i}-a^{*} x_{i} \geq b^{*}-\epsilon\right\}
\end{aligned}
$$

But then it holds that

$$
\begin{aligned}
\bar{f}\left(b^{*}-\epsilon\right)= & \sum_{i: y_{i}-a^{*} x_{i}<b^{*}}\left(b^{*}-\epsilon-\left(y_{i}-a^{*} x_{i}\right)\right) \\
& +\sum_{i: y_{i}-a^{*} x_{i} \geq b^{*}}\left(\left(y_{i}-a^{*} x_{i}\right)-b^{*}+\epsilon\right)
\end{aligned}
$$




$$
\begin{aligned}
= & \sum_{i: y_{i}-a^{*} x_{i}<b^{*}}\left(b^{*}-\left(y_{i}-a^{*} x_{i}\right)\right)+\sum_{i: y_{i}-a^{*} x_{i} \geq b^{*}}\left(\left(y_{i}-a^{*} x_{i}\right)-b^{*}\right) \\
& +\epsilon\left(\sum_{i: y_{i}-a^{*} x_{i} \geq b^{*}} 1-\sum_{i: y_{i}-a^{*} x_{i}<b^{*}} 1\right) \\
< & \bar{f}\left(b^{*}\right)+\epsilon\left(\frac{n}{2}-\frac{n}{2}\right) \\
= & \bar{f}\left(b^{*}\right)
\end{aligned}
$$

which contradicts the assumption of optimality of $b^{*}$.

" $\Leftarrow$ " Let $b^{*} \in \operatorname{median}\left\{\left(y_{i}-a^{*} x_{i}, 1\right)\right\}$. For $\epsilon>0$ small enough it can be shown that

$$
\bar{f}\left(b^{*}-\epsilon\right) \geq \bar{f}\left(b^{*}\right) \text { and } \bar{f}\left(b^{*}+\epsilon\right) \geq \bar{f}\left(b^{*}\right)
$$

similarly to the proof of the other direction. By convexity of $\bar{f}$ the optimality of $b^{*}$ follows.

Having everything we need at hand, we are able to prove the announced theorem.

Theorem I.2.2. A line $l^{*}$ optimal for $\left(P_{\text {ver }}(s)\right)$ is pseudo-halving.

Proof. Let $l^{*}=l_{a^{*}, b^{*}}=\left\{(x, y) \in \mathbb{R}^{2}: y=a^{*} x+b^{*}\right\}$ be an optimal solution to $\left(P_{\text {ver }}(s)\right)$, that is in particular $\left|a^{*}\right| \leq s$.

Keep $a^{*}$ fixed and look at the following function defined in $b$ :

$$
\bar{f}(b)=\sum_{i=1}^{n}\left|a^{*} x_{i}-y_{i}+b\right|
$$

Since $\left(a^{*}, b^{*}\right)$ is optimal to $\left(P_{\text {ver }}(s)\right)$ use

$$
f_{\text {ver }}\left(a^{*}, b\right)=\bar{f}(b) \forall b \in \mathbb{R}
$$

and conclude that $b^{*}$ minimizes $\bar{f}$. Therefore $b^{*}$ is a median of the set $\left\{\left(y_{i}-a^{*} x_{i}, 1\right)\right.$ : $i=1 \ldots n\}$ according to Proposition I.2.1,

By Definition I.2.3 it now holds that

$$
\sum_{i: y_{i}>a^{*} x_{i}+b} 1=\left|R\left(l_{a^{*}, b^{*}}\right)\right| \leq \frac{n}{2}
$$




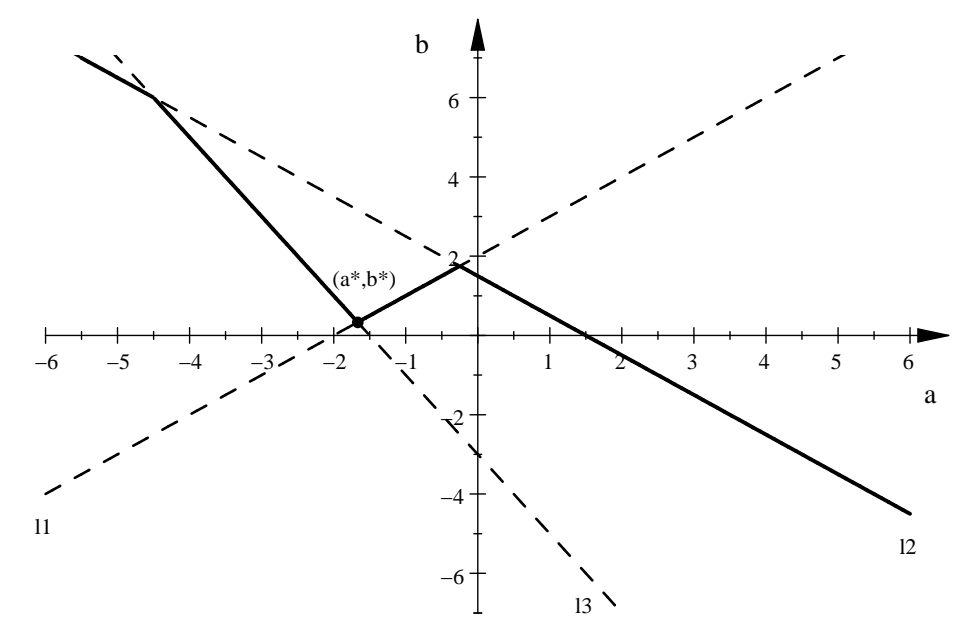

Figure I.2.3. The solid line segments give the path of pseudo-halving lines for Example I.1.1. One can see that the optimal solution $\left(a^{*}, b^{*}\right)$ to $\left(P_{v e r}\right)$ lies on the path.

$$
\text { and } \sum_{i: y_{i}<a^{*} x_{i}+b^{*}} 1=\left|L\left(l_{a^{*}, b^{*}}\right)\right| \leq \frac{n}{2} \text {. }
$$

This shows that $l^{*}$ is pseudo-halving.

We have shown that an optimal line for $\left(P_{\text {ver }}(s)\right)$ has to be pseudo-halving. This result allows a nice visualization of a new candidate set which is a subset of the set of all vertices in the dual space. The set of all pseudo-halving lines in the primal space gives a path in the dual space. We will refer to this path as the path of pseudo-halving lines. If $n$ is an odd number, this path is a sequence of segments of lines in $\mathcal{L}$ (cf. Figure [.2.3). If $n$ is even, there are segments of lines in $\mathcal{L}$ that bound the path of median lines from above and others that bound the path from below (cf. Figure I.2.4). The new candidate set is the set of all cell vertices that lie on the path of pseudo-halving lines.

In order to find an optimal solution for $\left(P_{v e r}(0)\right)$ we have to find out where the path of pseudo-halving lines intersects the $b$-axis of the dual space. Again we have to distinguish two cases:

Assume without loss of generality that the existing facilities $\left(x_{i}, y_{i}\right) \in \mathcal{E}$ are sorted in such a way that

$$
y_{(1)} \leq \ldots \leq y_{(n)} \text {. }
$$




\section{I.2. LOCATING LINES WITH VERTICAL DISTANCE AND A}

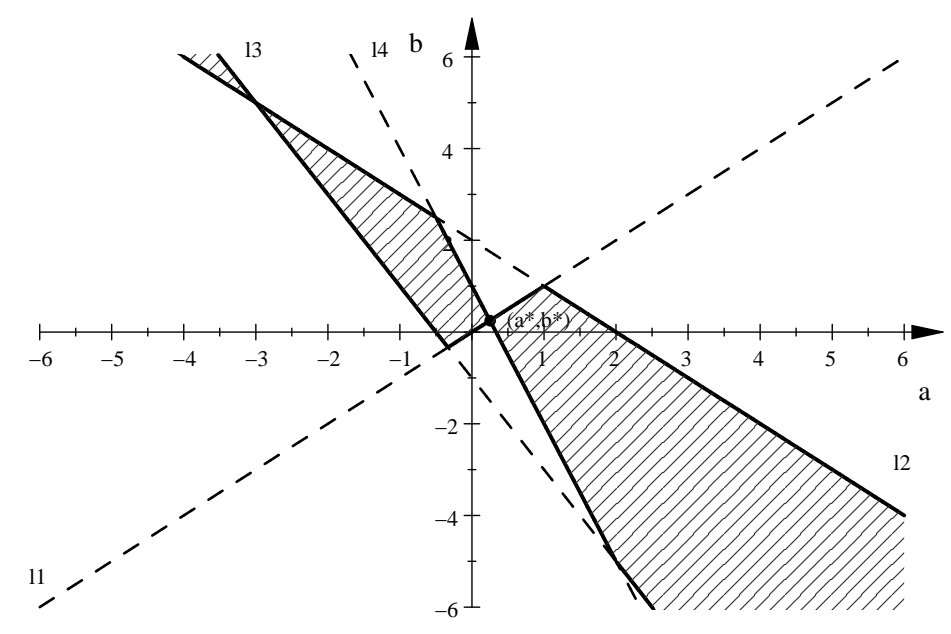

Figure I.2.4. The solid line segments plus the marked areas they are enclosing give the path of pseudo-halving lines for Example I.1.2. Again the optimal solution $\left(a^{*}, b^{*}\right)$ to $\left(P_{v e r}\right)$ lies on the path.

If the number of existing facilities $n$ is odd, an optimal solution to $\left(P_{\text {ver }}(0)\right)$ is the line $l_{0, y_{k^{*}}}$ with $y_{k^{*}}=y_{\left(\left\lceil\frac{n}{2}\right\rceil\right)}$. The value $y_{k^{*}}$ is exactly the median of the sorted sample $y_{(1)}, \ldots, y_{(n)}$.

On the other hand, if the number of existing facilities $n$ is even, the intersection $M$ of the path of pseudo-halving lines and the $b$-axis of the dual space is generally a set of several points. We can write it as

$$
M=\left\{(0, y) \in \mathbb{R}^{2}: y_{\left(\frac{n}{2}\right)} \leq y \leq y_{\left(\frac{n}{2}+1\right)}\right\}
$$

Any line $l_{0, y}$ with $(0, y) \in M$ is an optimal solution to $\left(P_{\text {ver }}(0)\right)$. This is due to the fact that $f_{v e r}\left(0, y_{\left(\frac{n}{2}\right)}\right)=f_{v e r}\left(0, y_{\left(\frac{n}{2}+1\right)}\right)$ and our problem is a convex optimization problem. The values of $y_{\left(\frac{n}{2}\right)}$ and $y_{\left(\frac{n}{2}+1\right)}$ correspond to what sometimes is called the lower median or the upper median of the sorted sample $y_{(1)}, \ldots, y_{(n)}$, respectively.

This means that we know how to locate a horizontal line in order to minimize the sum of vertical distances to the existing facilities. These conclusions are in analogy to the conclusions concerning 1-facility median problems in [Ham95]. We summarize them in a lemma.

Lemma I.2.1. If the number of existing facilities $n$ is odd, the line $l_{0, y_{\left(\left\lceil\frac{n}{2}\right\rceil\right)}}$ in the 


\section{LOCATING LINES WITH A RESTRICTION ON THE SLOPE USING VERTICAL DISTANCE}

primal space corresponding to the point $\left(0, y_{\left(\left\lceil\frac{n}{2}\right\rceil\right)}\right)$ in the dual space is optimal for $\left(P_{\text {ver }}(0)\right)$.

If the number of existing facilities $n$ is even, any line $l_{0, y}$ in the initial space corresponding to a point $(0, y)$ satisfying $y_{\left(\frac{n}{2}\right)} \leq y \leq y_{\left(\frac{n}{2}+1\right)}$ is optimal for $\left(P_{\text {ver }}(0)\right)$.

Proof. Nothing left to prove.

Now that we know a solution to our problem if $s=0$, we want to explore the behaviour of the optimal solutions if we increase $s$ little by little, meaning that we allow our optimal line to become steeper little by little. Our aim is to solve the problem for any $s \geq 0$, which in other words means that we want to know how the set of optimal solutions

$$
\begin{aligned}
M_{v e r}^{o p t}(s)= & \left\{\left(a^{*}(s), b^{*}(s)\right):\left|a^{*}(s)\right| \leq s \text { and } f_{\text {ver }}\left(a^{*}(s), b^{*}(s)\right) \leq f_{\text {ver }}(a, b)\right. \\
& \forall(a, b):|a| \leq s\}
\end{aligned}
$$

for our problem $\left(P_{\text {ver }}(s)\right)$ looks like for every positive $s$. Putting things together, we are interested in the set

$$
M^{*}:=\left\{M_{v e r}^{o p t}(s): s \geq 0\right\}
$$

Such a set is called an optimal location trajectory (see for example [BC91]). We will show that $M^{*}$ corresponds to a solution path in the dual space.

Assume that there exists a horizontal line $l_{0, b}$ that is optimal for the unrestricted line location problem $\left(P_{v e r}\right)$. Then a solution optimal for $\left(P_{v e r}(0)\right)$ stays optimal for $\left(P_{v e r}(s)\right)$ while we are increasing $s$. Obviously there can be more optimal solutions to $\left(P_{\text {ver }}(s)\right)$ than the ones we found for $\left(P_{v e r}(0)\right)$, but still we are done with this case since we at least know some optimal solution. In this case, our solution path in the dual space is a single point on the $b$-axis or a segment of the $b$-axis, depending on whether $n$ is odd or even. Therefore we will assume that there exists no horizontal line which is optimal for $\left(P_{v e r}\right)$ for the rest of this section, this means we assume $|a|>0$ for all lines $l_{a, b}$ optimal for $\left(P_{v e r}\right)$.

The assumption that there exists no optimal line which is horizontal in addition to the convexity of the objective function yields a nice statement about the optimal 


\section{I.2. LOCATING LINES WITH VERTICAL DISTANCE AND A

solutions of our unrestricted problem.

Theorem I.2.3. Let $M_{v e r}^{\text {opt }}:=\left\{\left(a^{*}, b^{*}\right): f_{\text {ver }}\left(a^{*}, b^{*}\right) \leq f_{\text {ver }}(a, b) \forall(a, b) \in \mathbb{R}^{2}\right\}$ and let $(0, b) \notin M_{\text {ver }}^{\text {opt }} \forall b \in \mathbb{R}$. Then either $a^{*}>0$ holds for all $\left(a^{*}, b^{*}\right) \in M_{\text {ver }}^{\text {opt }}$ or $a^{*}<0$ holds for all $\left(a^{*}, b^{*}\right) \in M_{\text {ver }}^{\text {opt }}$.

Proof. By assumption it is true that $(0, b) \notin M_{v e r}^{\text {opt }} \forall b \in \mathbb{R}$.

Let $B_{1}^{*}=\left(a_{1}^{*}, b_{1}^{*}\right), B_{2}^{*}=\left(a_{2}^{*}, b_{2}^{*}\right) \in M_{v e r}^{\text {opt }}$ with $a_{1}^{*}>0$ and $a_{2}^{*}<0$. The set $M_{v e r}^{\text {opt }}$ is convex since $\left(P_{\text {ver }}\right)$ is a convex problem. But then

$$
\lambda B_{1}^{*}+(1-\lambda) B_{2}^{*} \in M_{v e r}^{\text {opt }} \forall \lambda \in(0,1) .
$$

Choose $\lambda:=-\frac{a_{2}^{*}}{a_{1}^{*}-a_{2}^{*}}$. It can easily be shown that $\lambda \in(0,1)$ and therefore $\left(0, b^{\prime}\right) \in$ $M_{v e r}^{\text {opt }}$ with $b^{\prime}=\lambda b_{1}^{*}+(1-\lambda) b_{2}^{*}$, which is a contradiction to the assumption.

This means that if no optimal line is horizontal either all lines which are optimal for $\left(P_{v e r}\right)$ have positive slope or all lines which are optimal for $\left(P_{v e r}\right)$ have negative slope. In other words, there cannot exist $(a, b),\left(a^{\prime}, b^{\prime}\right) \in M_{v e r}^{o p t}$ with $a>0$ and $a^{\prime}<0$.

Suppose we know whether all optimal lines for the unrestricted line location problem have positive or negative slope. Can we use this information to make a statement about the sign of the slope of an optimal line for $\left(P_{v e r}(s)\right)$ ? The following theorem gives an answer to this question.

Theorem I.2.4. Let $M_{v e r}^{\text {opt }}(s)$ be the set of optimal solutions to $\left(P_{\text {ver }}(s)\right)$. If $a^{*}>0$ for all $\left(a^{*}, b^{*}\right) \in M_{\text {ver }}^{\text {opt }}$ then

$$
\left(a^{*}(s), b^{*}(s)\right) \in M_{\text {ver }}^{\text {opt }}(s) \Rightarrow a^{*}(s) \geq 0
$$

for all $s \geq 0$ Analogously it holds for all $s \geq 0$ that if $a^{*}<0$ for all $\left(a^{*}, b^{*}\right) \in M_{v e r}^{\text {opt }}$ then

$$
\left(a^{*}(s), b^{*}(s)\right) \in M_{v e r}^{\text {opt }}(s) \Rightarrow a^{*}(s) \leq 0
$$

Proof. Assume $a^{*}>0$ for all $\left(a^{*}, b^{*}\right) \in M_{v e r}^{\text {opt }}$. Let $\left(\hat{a}^{*}, \hat{b}^{*}\right) \in M_{v e r}^{\text {opt }}$.

If $s=0$ it holds that $a=0$ for all $(a, b)$ feasible to $\left(P_{v e r}(0)\right)$, therefore $a=0$ for all $(a, b) \in M_{v e r}^{\text {opt }}(0)$ in particular. 


\section{LOCATING LINES WITH A RESTRICTION ON THE SLOPE USING VERTICAL DISTANCE}

Assume that there exists a solution $\left(a^{*}(s), b^{*}(s)\right)$ to $\left(P_{s}\right)$ satisfying $a^{*}(s)<0$ for an arbitrary $s>0 . f_{v e r}$ is convex and $\left(\hat{a}^{*}, \hat{b}^{*}\right)$ is optimal to the unrestricted problem, that is to say for all $\lambda \in(0,1)$ it holds that

$$
\begin{aligned}
& f_{v e r}\left(\lambda a^{*}(s)+(1-\lambda) \hat{a}^{*}, \lambda b^{*}(s)+(1-\lambda) \hat{b}^{*}\right) \\
\leq & \lambda f_{v e r}\left(a^{*}(s), b^{*}(s)\right)+(1-\lambda) f_{v e r}\left(\hat{a}^{*}, \hat{b}^{*}\right) \\
\leq & \lambda f_{v e r}\left(a^{*}(s), b^{*}(s)\right)+(1-\lambda) f_{v e r}\left(a^{*}(s), b^{*}(s)\right) \\
= & f_{v e r}\left(a^{*}(s), b^{*}(s)\right)
\end{aligned}
$$

Choose $\lambda=-\frac{\hat{a}^{*}}{a^{*}(s)-\hat{a}^{*}}$. Then $\lambda \in(0,1)$ and the following equation holds:

$$
\begin{aligned}
f_{\text {ver }}\left(\lambda a^{*}(s)+(1-\lambda) \hat{a}^{*}, \lambda b^{*}(s)+(1-\lambda) \hat{b}^{*}\right) & =f_{\text {ver }}\left(0, \lambda b^{*}(s)+(1-\lambda) \hat{b}^{*}\right) \\
& \leq f_{\text {ver }}\left(a^{*}(s), b^{*}(s)\right)
\end{aligned}
$$

Assume

$$
f_{v e r}\left(0, \lambda b^{*}(s)+(1-\lambda) \hat{b}^{*}\right)=f_{v e r}\left(a^{*}(s), b^{*}(s)\right) .
$$

Then all inequalities above have to satisfy equality. Therefore it follows directly that

$$
f_{v e r}\left(\hat{a}^{*}, \hat{b}^{*}\right)=f_{v e r}\left(a^{*}(s), b^{*}(s)\right) .
$$

This is a contradiction to the assumption that $a>0 \forall(a, b) \in M_{v e r}^{\text {opt }}$, therefore

$$
f_{\text {ver }}\left(0, \lambda b^{*}(s)+(1-\lambda) \hat{b}^{*}\right)<f_{\text {ver }}\left(a^{*}(s), b^{*}(s)\right)
$$

must hold, which is a contradiction to the optimality of $\left(a^{*}(s), b^{*}(s)\right)$ since the solution $\left(0, \lambda b^{*}(s)+(1-\lambda) \hat{b}^{*}\right)$ is feasible for $\left(P_{v e r}(s)\right)$. The proposition follows.

In case $a^{*}<0$ for all $\left(a^{*}, b^{*}\right) \in M_{v e r}^{\text {opt }}$ the proposition can be proven analogously.

If an optimal solution for $\left(P_{v e r}\right)$ is known, the previous theorem makes sure that we only have to search one half-space for an optimal solution to $\left(P_{v e r}(s)\right)$ for all $s>0$, namely the half-space in which all optimal solutions of $\left(P_{v e r}\right)$ are included according to Theorem I.2.3. Therefore we can modify our candidate set again: The new candidate set is the set of all cell vertices that lie on that part of the path 


\section{I.2. LOCATING LINES WITH VERTICAL DISTANCE AND A RESTRICTION ON THE SLOPE}

of pseudo-halving lines which lies in the half-space containing all optimal solutions to the unrestricted line location problem.

Up to now we have reduced the candidate set for our restricted problem step by step. But what we really want to know is how to find an optimal solution for our problem $\left(P_{\text {ver }}(s)\right)$ for an arbitrary $s$. The following theorem states how this can be done.

Theorem I.2.5. If $a>0$ for all $(a, b) \in M_{v e r}^{\text {opt }}$, for any problem $\left(P_{\text {ver }}(s)\right)$ with $s \leq a^{*}:=\min \left\{a:(a, b) \in M_{v e r}^{\text {opt }}\right\}$ it holds that all optimal solutions $\left(a^{*}(s), b^{*}(s)\right)$ satisfy $a^{*}(s)=s$.

If $a<0$ for all $(a, b) \in M_{v e r}^{\text {opt }}$, for any problem $\left(P_{\text {ver }}(s)\right)$ with $s \leq a^{*}:=\min \{|a|$ : $\left.(a, b) \in M_{\text {ver }}^{\text {opt }}\right\}$ it holds that all optimal solutions $\left(a^{*}(s), b^{*}(s)\right)$ satisfy $a^{*}(s)=-s$.

Proof. First assume $a>0$ for all $(a, b) \in M_{\text {ver }}^{\text {opt }}$.

Let $0<s \leq a^{*}$ arbitrarily. We already know that all optimal lines of $\left(P_{v e r}(s)\right)$ have to have positive slope as well due to Theorem I.2.4. Assume there exists an $(\hat{a}, \hat{b}) \in M_{\text {ver }}^{\text {opt }}(s)$ with $\hat{a}<s$. If $s=a^{*}:=\min \left\{a:(a, b) \in M_{\text {ver }}^{\text {opt }}\right\}$ then $(\hat{a}, \hat{b})$ is an optimal solution to $\left(P_{v e r}\right)$ which is a contradiction to the choice of $a^{*}$.

Let therefore $0<s<a^{*}$. Since $\hat{a}<s$ and $a^{*}>s$ there exists a $\lambda \in(0,1)$ in such a way that

$$
\lambda \hat{a}+(1-\lambda) a^{*}=s
$$

But we know that $f_{v e r}$ is a convex function and therefore

$$
\begin{aligned}
f_{v e r}\left(\lambda \hat{a}+(1-\lambda) a^{*}, \lambda \hat{b}+(1-\lambda) b^{*}\right) & \leq \lambda f_{v e r}(\hat{a}, \hat{b})+(1-\lambda) f_{v e r}\left(a^{*}, b^{*}\right) \\
& <\lambda f_{v e r}(\hat{a}, \hat{b})+(1-\lambda) f_{v e r}(\hat{a}, \hat{b}) \\
& =f_{\text {ver }}(\hat{a}, \hat{b})
\end{aligned}
$$

which is a contradiction to the optimality of $(\hat{a}, \hat{b})$ since $\left(\lambda \hat{a}+(1-\lambda) a^{*}, \lambda \hat{b}+(1-\right.$ $\left.\lambda) b^{*}\right)$ is of course feasible for $\left(P_{v e r}(s)\right)$. Since $s$ has been chosen arbitrarily the proposition follows.

If $a<0$ for all $(a, b) \in M_{v e r}^{o p t}$ it is analogously shown that all $\left(a^{*}(s), b^{*}(s)\right) \in M_{v e r}^{\text {opt }}(s)$ satisfy $a^{*}(s)=-s$. 


\section{LOCATING LINES WITH A RESTRICTION ON THE SLOPE USING VERTICAL DISTANCE}

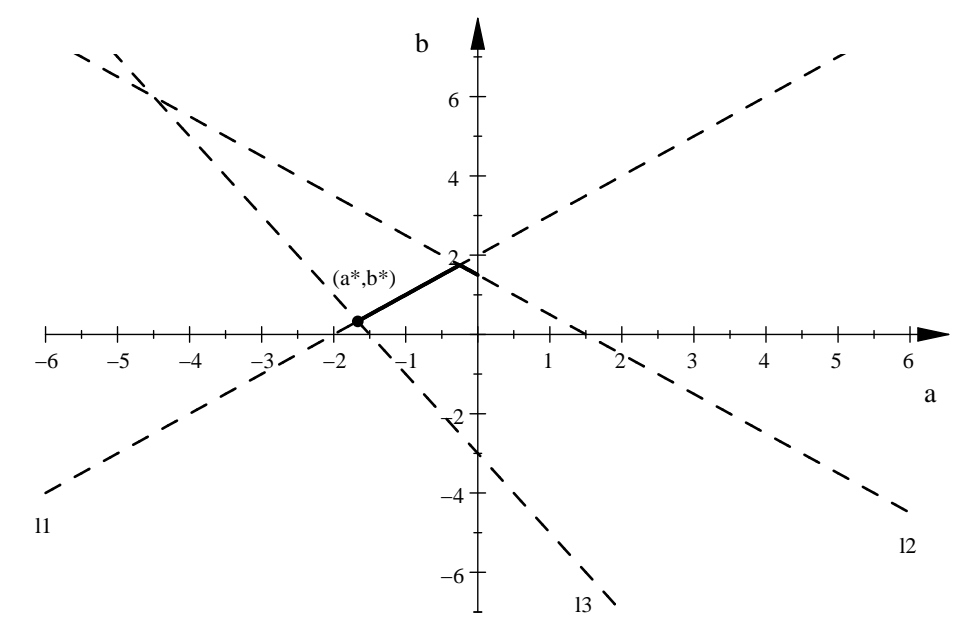

Figure I.2.5. The solid line segments give the solution path for Example I.1.1, For any $s>0$ we get an optimal solution to $\left(P_{\text {ver }}(s)\right)$ lying on this path. The path ends in the optimal solution $\left(a^{*}, b^{*}\right)$.

Putting our results of this section together, especially Lemma I.2.1 as well as Theorem 1.2 .4 and Theorem [.2.5, this results in a solution path for $\left(P_{v e r}(s)\right)$ in the dual space. If we want to solve $\left(P_{\text {ver }}(s)\right)$ for any $s \geq 0$, we only have to follow this path. It starts at the $b$-axis in the dual space with the intersection of the axis and the path of pseudo-halving lines (Lemma I.2.1). From there we move along the path of pseudo-halving lines towards $\left(a^{*}, b^{*}\right)$ as defined in Theorem I.2.5. The direction in which we move is well-defined due to Theorem I.2.4 and Theorem I.2.3. We follow this path until we reach an optimal solution for the unrestricted problem $\left(P_{v e r}\right)$, namely $\left(a^{*}, b^{*}\right)$. This is where our solution path ends since $\left(a^{*}, b^{*}\right)$ stays optimal for any $\left(P_{v e r}(s)\right)$ with $s>a^{*}$. Like this, the solution path enables us to give an optimal solution to $\left(P_{\text {ver }}(s)\right)$ for every $s$ (cf. Figures I.2.5 and I.2.6). This path is the visualization of the optimal location trajectory $M^{*}$ mentioned earlier in this section. Note that we can choose $(a(s), b(s)) \in M_{v e r}^{\text {opt }}, s \geq 0$, such that the corresponding optimal trajectory is continuous in $s$ and gives a unique solution for every $s$. Depending on whether the number of existing facilities is odd or even, we can choose the unique path of pseudo-halving lines or the unique path of lower median lines as such a continuous optimal trajectory, respectively.

While this is easy to see in the case of line location, we will see in Chapter $\mathrm{V}$ that it is not trivial to prove the existence of such a continuous optimal trajectory in 


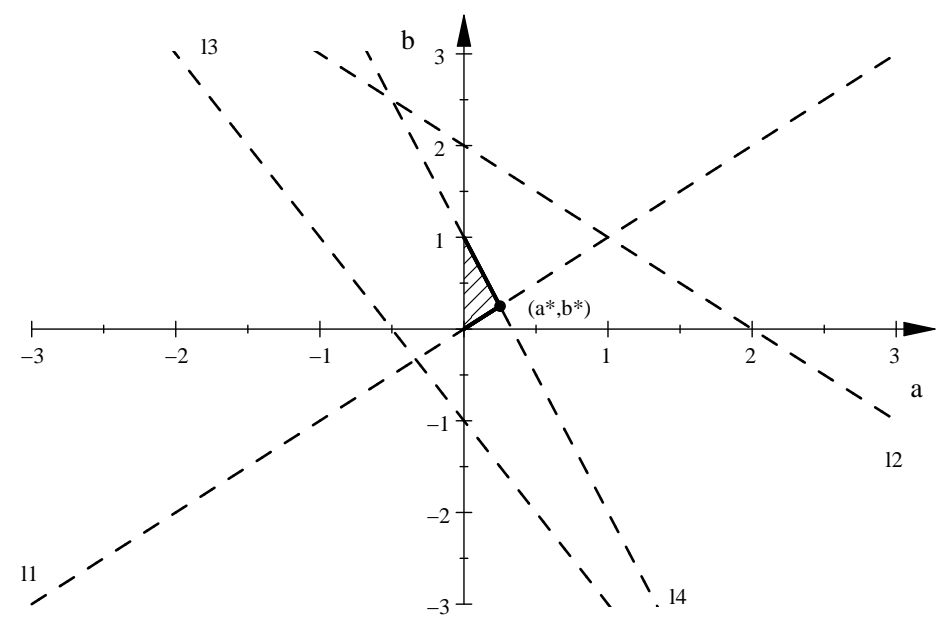

Figure I.2.6. The solid line segments plus the marked area they are enclosing give the solution path for Example I.1.2. For any $s>0$ we get either one optimal solution or a set of optimal solutions for $\left(P_{v e r}(s)\right)$ lying on this path. The path ends in the optimal solution $\left(a^{*}, b^{*}\right)$.

the more general case of hyperplane location with vertical distance. We will give a detailed proof for the existence of a continuous optimal trajectory in the general case in Section V.3.

Up to now, we have to know an optimal solution to $\left(P_{v e r}\right)$ in order to know in which direction the solution path runs. It would be preferable to have a method of finding out about the direction of the solution path without knowing an optimal solution. Therefore we end this section with the following theorem.

Theorem I.2.6. Let $\epsilon>0$ and let $\left(x_{k^{*}}, y_{k^{*}}\right)$ be an existing facility which is contained in an optimal line for $\left(P_{\text {ver }}(0)\right)$. Such a facility exists due to Lemma I.2.1 and it holds that

$$
k^{*}=\left\{\begin{array}{l}
\left(\left\lceil\frac{n}{2}\right\rceil\right) \text { if } n \text { is odd } \\
\left(\frac{n}{2}\right) \text { else. }
\end{array}\right.
$$

a) Let the number of existing facilities $n$ be odd.

$$
\text { If }
$$

$$
\sum_{i \in R\left(l_{0, y_{k^{*}}}\right)} x_{i}-\sum_{i \in L\left(l_{0, y_{k^{*}}}\right)} x_{i}<0
$$




\section{LOCATING LINES WITH A RESTRICTION ON THE SLOPE USING VERTICAL DISTANCE}

then

$$
f\left(-\epsilon, x_{k^{*}} \epsilon+y_{k^{*}}\right)<f\left(\epsilon,-x_{k^{*}} \epsilon+y_{k^{*}}\right)
$$

for some $\epsilon>0$. If otherwise

$$
\sum_{i \in R\left(l_{0, y_{k^{*}}}\right)} x_{i}-\sum_{i \in L\left(l_{0, y_{k^{*}}}\right)} x_{i}>0
$$

then

$$
f\left(\epsilon,-x_{k^{*}} \epsilon+y_{k^{*}}\right)<f\left(-\epsilon, x_{k^{*}} \epsilon+y_{k^{*}}\right)
$$

for some $\epsilon>0$ accordingly.

b) Let the number of existing facilities $n$ now be even.

If

$$
\sum_{i \in R\left(l_{0, y_{k^{*}}}\right)} x_{i}-\sum_{i \in L\left(l_{0, y_{k^{*}}}\right)} x_{i}<\left|x_{k^{*}}\right|
$$

then

$$
f\left(-\epsilon, x_{k^{*}} \epsilon+y_{k^{*}}\right)<f\left(\epsilon,-x_{k^{*}} \epsilon+y_{k^{*}}\right)
$$

for some $\epsilon>0$. If otherwise

$$
\sum_{i \in R\left(l_{0, y_{k^{*}}}\right)} x_{i}-\sum_{i \in L\left(l_{0, y_{k^{*}}}\right)} x_{i}>\left|x_{k^{*}}\right|
$$

then

$$
f\left(\epsilon,-x_{k^{*}} \epsilon+y_{k^{*}}\right)<f\left(-\epsilon, x_{k^{*}} \epsilon+y_{k^{*}}\right)
$$

for some $\epsilon>0$ accordingly.

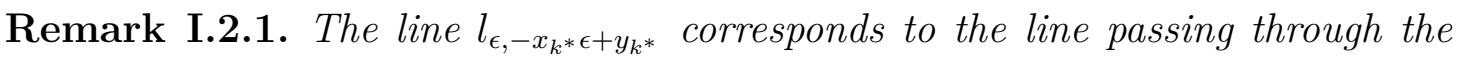
point $\left(x_{k^{*}}, y_{k^{*}}\right)$ with slope $\epsilon$. It therefore corresponds to the line one gets by twisting the line optimal to $\left(P_{\text {ver }}(0)\right)$ in the point $\left(x_{k^{*}}, y_{k^{*}}\right)$ a little bit.

Analogously $l_{-\epsilon, x_{k^{*}}+y_{k^{*}}}$ corresponds to the line one gets by twisting the line optimal to $\left(P_{\text {ver }}(0)\right)$ in the point $\left(x_{k^{*}}, y_{k^{*}}\right)$ a little bit in the opposite direction.

Starting with $a=0$ and restricting the slope by $|\epsilon|$, Theorem I.2.5 guarantees that there exists an optimal line with slope $\epsilon$ or $-\epsilon$, respectively. In fact there exists 


\section{I.2. LOCATING LINES WITH VERTICAL DISTANCE AND A RESTRICTION ON THE SLOPE}

an optimal solution to $\left(P_{\text {ver }}(\epsilon)\right)$ lying on the median path, that is to say it exists an optimal line with slope $\epsilon$ or $-\epsilon$, respectively, still passing through the point $\left(x_{k^{*}}, y_{k^{*}}\right)$ since we can chose $\epsilon$ sufficiently small so that there is no intersection $(a, b)$ between two of the lines in the dual space induced by the existing facilities with $0<|a| \leq \epsilon$. Therefore either $l_{-\epsilon, x_{k^{*}} \epsilon+y_{k^{*}}}$ or $l_{\epsilon,-x_{k^{*}} \epsilon+y_{k^{*}}}$ has to be an optimal line. Since there is no intersection $(a, b)$ between two of the lines in the dual space satisfying $|a| \leq \epsilon$, it holds that

$$
R\left(l_{\epsilon,-x_{k^{*}} \epsilon+y_{k^{*}}}\right)=R\left(l_{0, y_{k^{*}}}\right), \quad L\left(l_{\epsilon,-x_{k^{*}} \epsilon+y_{k^{*}}}\right)=L\left(l_{0, y_{k^{*}}}\right)
$$

and

$$
R\left(l_{-\epsilon, x_{k^{*}} \epsilon+y_{k^{*}}}\right)=R\left(l_{0, y_{k^{*}}}\right), \quad L\left(l_{-\epsilon, x_{k^{*}} \epsilon+y_{k^{*}}}\right)=L\left(l_{0, y_{k^{*}}}\right) .
$$

This theorem provides information about which one of these two lines is optimal. At the same time it provides an even stronger result: Since all optimal solutions to the problems $\left(P_{\text {ver }}(s)\right)$ with $s \geq 0$ have the same sign according to Theorem I.2.4, this theorem provides information about the direction of the solution path with increasing $s$.

Proof. Let $n$ be odd. In order to simplify the notation we write $R$ and $L$ instead of $R\left(l_{0, y_{k^{*}}}\right)$ and $L\left(l_{0, y_{k^{*}}}\right)$, respectively.

$$
\begin{array}{ccc} 
& \sum_{i \in R} x_{i}-\sum_{i \in L} x_{i}<0 . \\
& \sum_{i \in R} x_{i}-\sum_{i \in L} x_{i}<(|R|-|L|) x_{k^{*}} \\
\Rightarrow & \sum_{i \in R}\left(x_{i}-x_{k^{*}}\right)-\sum_{i \in L}\left(x_{i}-x_{k^{*}}\right) & <0 \\
\Rightarrow & 2 \cdot\left(\sum_{i \in R}\left(x_{i}-x_{k^{*}}\right)-\sum_{i \in L}\left(x_{i}-x_{k^{*}}\right)\right) & <0 \\
\Rightarrow & \sum_{i \in R}\left(x_{i}-x_{k^{*}}\right)-\sum_{i \in L}\left(x_{i}-x_{k^{*}}\right) & <\sum_{i \in L}\left(x_{i}-x_{k^{*}}\right)-\sum_{i \in R}\left(x_{i}-x_{k^{*}}\right)
\end{array}
$$




$$
\begin{aligned}
& \Rightarrow \quad \epsilon \cdot\left(\sum_{i \in R}\left(x_{i}-x_{k^{*}}\right)-\sum_{i \in L}\left(x_{i}-x_{k^{*}}\right)\right)-|R| y_{k^{*}}+|L| y_{k^{*}}+\sum_{i \in R} y_{i}-\sum_{i \in L} y_{i} \\
& <\epsilon \cdot\left(\sum_{i \in L}\left(x_{i}-x_{k^{*}}\right)-\sum_{i \in R}\left(x_{i}-x_{k^{*}}\right)\right)-|R| y_{k^{*}}+|L| y_{k^{*}}+\sum_{i \in R} y_{i}-\sum_{i \in L} y_{i} \\
& \Rightarrow \quad \sum_{i \in R}\left(y_{i}+\epsilon x_{i}-\epsilon x_{k^{*}}-y_{k^{*}}\right)+\sum_{i \in L}\left(-y_{i}-\epsilon x_{i}+\epsilon x_{k^{*}}+y_{k^{*}}\right) \\
& <\sum_{i \in R}\left(y_{i}-\epsilon x_{i}+\epsilon x_{k^{*}}-y_{k^{*}}\right)+\sum_{i \in L}\left(-y_{i}+\epsilon x_{i}-\epsilon x_{k^{*}}+y_{k^{*}}\right) \\
& \stackrel{(* *)}{\Rightarrow} \\
& \sum_{i \in R} d_{v e r}\left(\left(x_{i}, y_{i}\right), l_{-\epsilon, x_{k^{*}} \epsilon+y_{k^{*}}}\right)+\sum_{i \in L} d_{v e r}\left(\left(x_{i}, y_{i}\right), l_{-\epsilon, x_{k^{*}} \epsilon+y_{k^{*}}}\right) \\
& <\sum_{i \in R} d_{v e r}\left(\left(x_{i}, y_{i}\right), l_{\epsilon,-x_{k^{*}} \epsilon+y_{k^{*}}}\right)+\sum_{i \in L} d_{v e r}\left(\left(x_{i}, y_{i}\right), l_{\epsilon,-x_{k^{*}} \epsilon+y_{k^{*}}}\right) \\
& \Rightarrow \quad f\left(l_{-\epsilon, x_{k^{*}} \epsilon+y_{k^{*}}}\right)<f\left(l_{\epsilon,-x_{k^{*}} \epsilon+y_{k^{*}}}\right)
\end{aligned}
$$

Here $(*)$ holds because of the fact that $|R|=|L|$ and (**) holds because of the fact that

$$
R\left(l_{\epsilon,-x_{k^{*}} \epsilon+y_{k^{*}}}\right)=R, L\left(l_{\epsilon,-x_{k^{*}} \epsilon+y_{k^{*}}}\right)=L
$$

as well as

$$
R\left(l_{-\epsilon, x_{k^{*}} \epsilon+y_{k^{*}}}\right)=R, L\left(l_{-\epsilon, x_{k^{*}} \epsilon+y_{k^{*}}}\right)=L,
$$

as we already mentioned above.

The case

$$
\sum_{i \in R} x_{i}-\sum_{i \in L} x_{i}>0
$$

can be proven analogously.

Let $n$ be even now and let

$$
\begin{aligned}
& \sum_{i \in R} x_{i}-\sum_{i \in L} x_{i}<x_{k^{*}} . \\
\Rightarrow & \sum_{i \in R} x_{i}-\sum_{i \in L} x_{i}<(|R|-|L|) x_{k^{*}}
\end{aligned}
$$

because of the fact that $|R|=|L|+1$. The proposition $b$ ) can now be proven in 
an analogous manner to a).

Likewise the case

$$
\sum_{i \in R} x_{i}-\sum_{i \in L} x_{i}>x_{k^{*}}
$$

can be proven analogously.

This theorem not only allows us to check in which direction the continuous solution path of $\left(P_{v e r}(s)\right)$ will proceed, it implicitly provides a method of checking what sign an optimal solution for $\left(P_{v e r}\right)$ does have as well as of finding such an optimal solution by following the solution path until we get stuck in some point which then corresponds to an optimal line.

Example I.2.3. Consider one more time the existing facilities in Example I.1.2. We have $l_{0, y_{k^{*}}}=l_{0,0}$ and therefore $R\left(l_{0, y_{k^{*}}}\right)=\left\{x_{2}, x_{4}\right\}$ while $L\left(l_{0, y_{k^{*}}}\right)=\left\{x_{3}\right\}$. This gives us

$$
\sum_{i \in R\left(l_{\left.0, y_{k^{*}}\right)}\right.} x_{i 1}-\sum_{i \in L\left(l_{0, y_{k^{*}}}\right)} x_{i 1}=1+3-2=2>1=\left|x_{k^{*}}\right|
$$

which means that our solution path runs in the positive direction. This result is in agreement with Figure I.2.6. 


\section{Chapter II}

\section{An excursion to statistics: RLAD regression and its relation to locating lines and hyperplanes}

We already mentioned in Section 0.1 that there exists an interest in line location in different fields of mathematics. In this chapter we want to present an application in statistics for which we can use our results about line location with vertical distance.

Linear regression is a field of statistics in which results about line location are very useful. As a matter of fact, the problem of finding a regression line is nothing else than the problem of locating a line using a certain function to measure the distance between the given points and the line. A lot of publications have considered problems of this type, for example [Ric64, Sha71, PFTV86, SW87, Edg88, GP02]. We want to present a regression method, namely the Regularized Least Absolute Deviations (RLAD) regression introduced by [WGZ06], and point out that the optimal trajectory of the problem of locating a line with vertical distance with a restriction on the slope we developed in the previous section provides in fact a path of optimal estimators for RLAD regression.

Linear regression is an important field in data analysis. We face the following problem: A variable $Y$ is supposed to be described through a vector $x \in \mathbb{R}^{k}$ of $k$ independent predictor variables. Then $Y$ is called the response variable. Linear 


\section{AN EXCURSION TO STATISTICS: RLAD REGRESSION AND 42 ITS RELATION TO LOCATING LINES AND HYPERPLANES}

regression assumes the following linear relationship between $Y$ and $x$ :

$$
Y=\beta_{k} x_{k}+\ldots+\beta_{1} x_{1}+\beta_{0}+\epsilon,
$$

where $\epsilon \in \mathbb{R}$ is a random non-observable error and $\beta_{0}, \ldots, \beta_{k} \in \mathbb{R}$ are unknown parameters. The aim is to estimate the unknown parameters. Suppose we obtain $n$ samples to do so. Let $y=\left(y_{1}, \ldots, y_{n}\right)^{t} \in \mathbb{R}^{n}$ be the vector of the $n$ observations for the response variable $Y$ and $x_{1}=\left(x_{11}, \ldots, x_{n 1}\right)^{t}, \ldots, x_{k}=\left(x_{1 k}, \ldots, x_{n k}\right)^{t} \in \mathbb{R}^{n}$ the $n$ different observations for the $k$ different predictor variables. We can summarize the observations for the predictor variables in an $n \times k$-matrix $X \in \mathbb{R}^{n \times k}$ where each row corresponds to a sample and each column corresponds to a predictor, that is to say $X=\left[x_{1} \ldots x_{k}\right]$. Now linear regression assumes

$$
y=e \beta_{0}+X \beta+\epsilon,
$$

where $e=(1, \ldots, 1)^{t} \in \mathbb{R}^{n}, \beta=\left(\beta_{1}, \ldots, \beta_{k}\right)^{t}$ and $\epsilon \in \mathbb{R}^{n}$ being a stochastical error. From now on, we denote by $[X e] \in \mathbb{R}^{n \times(k+1)}$ the $n \times(k+1)$-matrix consisting of the matrix $X$ and the vector $e$ added in the last column. If $\epsilon$ has normal distribution with constant variance and is independent of $x$, the well-known Ordinary Least Squares (OLS) estimator provides an optimal estimator of $\beta$ and $\beta_{0}$ by minimizing the sum of the $l_{2}$-norm of the residuals:

$$
\left(\begin{array}{c}
\hat{\beta} \\
\hat{\beta}_{0}
\end{array}\right)=\arg \min \left(\left\|y-[X e]\left(\begin{array}{c}
\beta \\
\beta_{0}
\end{array}\right)\right\|_{2}\right)^{2},
$$

There are two main difficulties linked to the OLS.

One problem is that the OLS is very sensitive to outliers which can lead to very bad results if $\epsilon$ is not normally distributed, e.g. if it has heavy-tailed distribution. This is why robust methods minimize other functions of residuals than the $l_{2}$ norm. One example for a robust method is the Least Absolute Deviations (LAD) regression, which minimizes the $l_{1}$-norm of the residuals: 


\section{AN EXCURSION TO STATISTICS: RLAD REGRESSION AND ITS RELATION TO LOCATING LINES AND HYPERPLANES 43}

$$
\left(\begin{array}{c}
\hat{\beta} \\
\hat{\beta}_{0}
\end{array}\right)=\arg \min \left\|y-[X e]\left(\begin{array}{c}
\beta \\
\beta_{0}
\end{array}\right)\right\|_{1} .
$$

A number of works have confirmed that LAD estimation is more robust to outliers than the OLS estimator, e.g. [Sha71, HJKP90, MM01, GP02, GP04].

Another problem is that linear regression often leads to coefficients which have low bias but large variance (see for example [Tib96]). Therefore it is tried to give up a little bias to reduce the variance of the estimator by shrinkage methods in order to improve the overall performance of the estimator. One of these shrinkage methods is the so-called Least Absolute Shrinkage and Selection Operator (LASSO) introduced by [Tib96]. It minimizes a function that consists of the $l_{2}$-norm of the residuals and a penalty term:

$$
\left(\begin{array}{c}
\hat{\beta} \\
\hat{\beta}_{0}
\end{array}\right)=\arg \min \left(\left\|y-[X e]\left(\begin{array}{c}
\beta \\
\beta_{0}
\end{array}\right)\right\|_{2}\right)^{2}+\lambda\|\beta\|_{1} .
$$

Even if it is controversial whether the overall performance of the estimator can be improved in general or not, this regularization method has a nice property: Some of the coefficients can be shrunk exactly to 0 when $\lambda$ is chosen properly due to the $l_{1}$-penalty on the coefficient vector. This is what is called an automatic feature selection (see for example [Tib96]).

As an alternative, [WGZ06] introduced the so-called Regularized Least Absolute Deviations (RLAD) regression in a try to combine the pleasant features of the LAD estimator and the LASSO. RLAD regression solves the following optimization problem:

$$
\min \| \underbrace{y-[X e]\left(\begin{array}{c}
\beta \\
\beta_{0}
\end{array}\right) \|_{1}}_{\text {LAD-part }}+\underbrace{\lambda\|\beta\|_{1}}_{\text {LASSO-part }}, \quad\left(P_{R L A D}(\lambda)\right)
$$

where $\lambda \geq 0$ is a tuning parameter.

Due to the LAD part, the RLAD estimator is less sensitive to outliers. The LASSO part effects that it can perform automatic feature selection. Again a little bias might be traded against a lower variance in the hope of improving the overall performance. 


\section{AN EXCURSION TO STATISTICS: RLAD REGRESSION AND 44 ITS RELATION TO LOCATING LINES AND HYPERPLANES}

A problem of the LASSO and therefore as well of the RLAD is that it is not clear how to choose $\lambda$ in order to get a good estimator. The quality of the estimator can vary significantly with the choice of a different $\lambda$. Therefore statisticians are especially interested in analysing how solutions to $\left(P_{R L A D}(\lambda)\right)$ behave for different values of $\lambda$.

We now want to look at RLAD regression as a bicriterial optimization problem. Using well-known results from multicriterial optimization, we will be able to show that we can use the results of the previous section in order to give optimal RLAD regression lines for any $\lambda$. For an introduction into the field of multicriterial optimization see for example [Ehr05]. We consider the following bicriterial problem:

$$
\min _{\left(\beta, \beta_{0}\right) \in \mathbb{R}^{k+1}} f\left(\beta, \beta_{0}\right),
$$

where $f=\left(f_{1}, f_{2}\right)$ with

$$
f_{1}\left(\beta, \beta_{0}\right)=\left\|y-[X e]\left(\begin{array}{c}
\beta \\
\beta_{0}
\end{array}\right)\right\|_{1}
$$

and

$$
f_{2}\left(\beta, \beta_{0}\right)=\|\beta\|_{1}
$$

This means that $f_{1}$ represents the LAD-part of the problem while $f_{2}$ represents its LASSO-part. These two objective functions are conflicting with each other: Obviously, for $f_{2}$ it would be best to chose $\beta$ equal to zero, but that could lead to a very bad function value of $f_{1}$. Therefore it is not obvious how to define an "optimal" solution for the bicriterial problem. In multicriterial optimization, the concept of so called Pareto optimal solutions is often used.

Definition II.0.4. (Ehr05]) A solution $b^{*}=\left(\beta^{*}, \beta_{0}{ }^{*}\right)^{t} \in \mathbb{R}^{k+1}$ is called Pareto optimal to $\left(P_{b i}\right)$ if there is no $b=\left(\beta, \beta_{0}\right)^{t} \in \mathbb{R}^{k+1}$ such that $f_{i}(b) \leq f_{i}\left(b^{*}\right), i=1,2$, and $f_{i}(b)<f_{i}\left(b^{*}\right)$ for an $i \in\{1,2\}$.

Furthermore, it is often distinguished between weakly Pareto optimal points and strictly Pareto optimal points. Therefore we introduce these notions as well.

Definition II.0.5. ([Ehr05]) $b^{*}=\left(\beta^{*}, \beta_{0}{ }^{*}\right) \in \mathbb{R}^{k+1}$ is called weakly Pareto opti- 


\section{AN EXCURSION TO STATISTICS: RLAD REGRESSION AND ITS RELATION TO LOCATING LINES AND HYPERPLANES 45}

mal if there is no $b=\left(\beta, \beta_{0}\right) \in \mathbb{R}^{k+1}$ such that $f(b)<f\left(b^{*}\right)$, i.e. $f_{i}(b)<f_{i}\left(b^{*}\right)$ for $i=1,2$.

$b^{*}=\left(\beta^{*}, \beta_{0}{ }^{*}\right) \in \mathbb{R}^{k+1}$ is called strictly Pareto optimal if there is no $b=\left(\beta, \beta_{0}\right) \in$ $\mathbb{R}^{k+1}, b \neq b^{*}$ such that $f(b) \leq f\left(b^{*}\right)$.

In multicriterial optimization, there exist several concepts of how to find Pareto optimal solutions. One of these concepts is the so called weighted sum scalarization. If we look at our problem $\left(P_{b i}\right)$, weighted sum scalarization works like this: Instead of looking at our bicriterial problem $\left(P_{b i}\right)$ we now put a nonnegative weight $\lambda_{i} \in$ $\mathbb{R}_{+}, i=1,2$, on each of our functions $f_{1}$ and $f_{2}$ and minimize the weighted sum of these two functions. Let $\Lambda=\left(\lambda_{1}, \lambda_{2}\right) \in \mathbb{R}_{+}^{2}$ be the vector of weights, then the weighted sum scalarization of our problem $\left(P_{b i}\right)$ looks like this:

$$
\min _{\left(\beta, \beta_{0}\right)^{t} \in \mathbb{R}^{k+1}} \lambda_{1} \cdot f_{1}\left(\beta, \beta_{0}\right)+\lambda_{2} \cdot f_{2}\left(\beta, \beta_{0}\right)
$$

Note that if we put $\lambda_{1}=1$ and $\lambda_{2}=\lambda$ with $\lambda \geq 0$ we get exactly the problem $\left(P_{R L A D}(\lambda)\right)$. Now we can use the following theorem to identify the optimal solutions to the RLAD problem as weakly Pareto optimal solutions for $\left(P_{b i}\right)$.

Theorem II.0.7. ([Ehr05]) Let $\left(P_{b i}\right)$ be a bicriterial problem and $(P(\Lambda))$ the corresponding sum scalarization problem. If the objective space of $(P(\Lambda))$ is convex and $\lambda \in \mathbb{R}_{+}^{2} \backslash\{0\}$, then the optimal solutions for $(P(\Lambda))$ are weakly Pareto optimal solutions for $\left(P_{b i}\right)$.

The assumptions of this theorem are satisfied by our problem $\left(P_{R L A D}(\lambda)\right)$ since the objective space is obviously convex and $\Lambda=(1, \lambda)$ with $\lambda \geq 0$ is an element of $\mathbb{R}_{+}^{2} \backslash\{0\}$. As we already mentioned this problem is a weighted sum scalarization of our problem $\left(P_{b i}\right)$, therefore the optimal solutions to $\left(P_{R L A D}(\lambda)\right)$ are weakly Pareto optimal for $\left(P_{b i}\right)$.

Another concept for finding Pareto optimal solutions is the $\epsilon$-constraint method. It was introduced by [HLW71]. Applying this method to our problem $\left(P_{b i}\right)$, we only minimize one of the two functions $f_{1}$ and $f_{2}$ whereas a constraint is put on the other one. In our case, we are interested in minimizing $f_{1}$ while putting a 


\section{AN EXCURSION TO STATISTICS: RLAD REGRESSION AND 46 ITS RELATION TO LOCATING LINES AND HYPERPLANES}

constraint on $f_{2}$. The resulting problem looks like this:

$$
\begin{aligned}
& \min f_{1}\left(\beta, \beta_{0}\right) \\
& \text { s.t. } f_{2}\left(\beta, \beta_{0}\right) \leq \epsilon,
\end{aligned}
$$

where $\epsilon \in \mathbb{R}$. The next theorem shows that we can find optimal solutions to $\left(P_{R L A D}(\lambda)\right)$ for arbitrary $\lambda \geq 0$ by solving $(P(\epsilon))$ for every $\epsilon \geq 0$.

Theorem II.0.8. (see e.g. [Ehr05])

a) Suppose $\left(\hat{\beta}, \hat{\beta}_{0}\right)$ is a solution to $\min _{\left(\beta, \beta_{0}\right) \in \mathbb{R}^{k+1}} \lambda_{1} \cdot f_{1}\left(\beta, \beta_{0}\right)+\lambda_{2} \cdot f_{2}\left(\beta, \beta_{0}\right)$. If $\lambda_{1}>0$ there exists a $\hat{\epsilon}$ so that $\left(\hat{\beta}, \hat{\beta}_{0}\right)$ solves the problem $(P(\epsilon))$.

b) If $\left(\hat{\beta}, \hat{\beta}_{0}\right)$ solves the problem $(P(\hat{\epsilon}))$ there exists a $\Lambda \in \mathbb{R}_{+}^{2} \backslash\{0\}$ so that $\left(\hat{\beta}, \hat{\beta}_{0}\right)$ solves the problem $(P(\Lambda))$.

Since in our problem $\left(P_{R L A D}(\lambda)\right)$ we have $\lambda_{1}=1$ fixed, this theorem ensures that we are able to find optimal solutions to $\left(P_{R L A D}(\lambda)\right)$ with $\lambda \geq 0$ by solving $(P(\epsilon))$ with a certain $\epsilon$.

If we now name the constraint $s$ instead of $\epsilon$, we know that the following problem is an equivalent formulation of $\left(P_{R L A D}(\lambda)\right)$, therefore we will denote it by $\left(P_{R L A D}(s)\right)$ :

$$
\begin{aligned}
& \min \left\|y-[X e]\left(\begin{array}{c}
\beta \\
\beta_{0}
\end{array}\right)\right\|_{1} \quad\left(P_{R L A D}(s)\right) \\
& \text { s.t. }\|\beta\|_{1} \leq s
\end{aligned}
$$

with $s \geq 0$. Every optimal solution to $\left(P_{R L A D}(s)\right)$ corresponds to an optimal solution to $\left(P_{R L A D}(\lambda)\right)$ with a certain $\lambda$.

Let us now consider $\left(P_{R L A D}(s)\right)$ for $k=1$, e.g. there exists only one predictor variable $x$ and therefore only one coefficient $\beta \in \mathbb{R}$. We assume again that we obtain $n$ samples. Let $y=\left(y_{1}, \ldots, y_{n}\right)$ be the vector of observations for the 


\section{AN EXCURSION TO STATISTICS: RLAD REGRESSION AND ITS RELATION TO LOCATING LINES AND HYPERPLANES 47}

response variable and $x=\left(x_{1}, \ldots, x_{n}\right)$ the vector of observations for the predictor variable. We get the optimization problem

$$
\begin{array}{ll}
\min & \left\|y-x \beta-e \beta_{0}\right\|_{1} \quad\left(P_{R L A D}(s)\right) \\
\text { s.t. }|\beta| \leq s &
\end{array}
$$

or equivalently

$$
\begin{array}{ll}
\min & \sum_{i=1}^{n}\left|y_{i}-\beta x_{i}-\beta_{0}\right| \quad\left(P_{R L A D}(s)\right) \\
\text { s.t. }|\beta| \leq s &
\end{array}
$$

with $s \geq 0$.

This is the problem of locating a RLAD regression line $l_{\beta, \beta_{0}}$ in the plane with a restriction on the slope, namely $|\beta| \leq s$, in such a way that the sum of vertical distances to a given set of data points $\left\{\left(x_{1}, y_{1}\right), \ldots,\left(x_{n}, y_{n}\right)\right\}$ is minimized. This corresponds exactly to our problem of locating a line with vertical distance and a restriction on the slope. If we let $\beta=a$ and $b=\beta_{0}$ as well as $\mathcal{E}=\left\{\left(x_{1}, y_{1}\right), \ldots,\left(x_{n}, y_{n}\right)\right\}$ then $\left(P_{R L A D}(s)\right)$ and $\left(P_{v e r}(s)\right)$ are identical problems. In fact, $\left(P_{v e r}\right)$ and $\left(P_{L A D}\right)$ are identical as well. Therefore the solution path we provided for $\left(P_{\text {ver }}(s)\right)$ in Chapter $\llbracket$ is also a solution path for RLAD regression with $k=1$.

Note that for the RLAD regression, Theorem I.2.4 in Section I.2 is particularly interesting. As we mentioned earlier in this chapter, statisticians are interested in solving $\left(P_{R L A D}(s)\right)$ for any $s \geq 0$ since it is not clear how to choose $s$ in order to get a good estimator in the first place. Having Theorem I.2.4 at hand, it is still not clear how to choose $s$ but at least we can be sure that our RLAD regression line has the same sign in slope as a LAD regression line no matter how we choose the $s$.

Up to now we only stressed the equivalence between the problem of finding a RLAD regression line and the problem of locating a line with a restriction on the slope. Comparing $\left(P_{R L A D}(s)\right)$ for arbitrary $k$ to the formulation of the hyperplane 


\section{AN EXCURSION TO STATISTICS: RLAD REGRESSION AND 48 ITS RELATION TO LOCATING LINES AND HYPERPLANES}

location problem with a restriction on the slope $\left(P_{v e r}(s)\right)$ in Chapter $\mathbf{Q}$, it is easy to see that the above equivalence holds for any $k$. In other words, the problem of locating a RLAD regression hyperplane is equivalent to the problem of locating a hyperplane with a restriction on the slope using the vertical distance. This is not a surprise since the location of a hyperplane with a restriction on the slope is a generalization of the problem of locating a line with a restriction on the slope. Nevertheless, it is a nice fact that the solution path we construct in Section V.4 is a solution path for RLAD regression in the $\mathbb{R}^{k+1}$ as well. 


\section{Chapter III}

\section{Locating lines with a restriction on the slope using horizontal distance}

In Chapter I we dealt with line location using vertical distance when there is a restriction on the slope of the line. As we already mentioned we want to look at line location problems using different kinds of distances. In this chapter, we consider line location problems with horizontal distance $d_{h o r}$ and a restriction on the slope.

Our aim is to provide a solution path like we did for $\left(P_{v e r}(s)\right)$. Therefore we first introduce the horizontal distance and the problem that we will consider. We will see that there is a strong connection between the horizontal distance and the vertical distance which we can put to account in order to apply the results we have presented in Chapter II. This is why we will analyse the relationship between line location with vertical distance and line location with horizontal distance when there is a restriction on the slope in both cases. 


\section{III.1 Locating lines with horizontal distance and a restriction on the slope}

We start with the definition of the horizontal distance between two points as well as between a point and a line.

Definition III.1.1. (See for example [Sch99])

Let $x, y \in \mathbb{R}^{2}$. Then we define the horizontal distance between $x$ and $y$ as

$$
d_{\text {hor }}(x, y)=\left\{\begin{array}{l}
\left|y_{1}-x_{1}\right| \text { if } x_{2}=y_{2} \\
\infty \text { otherwise }
\end{array}\right.
$$

The horizontal distance between a point $x \in \mathbb{R}^{2}$ and a line $l$ we define as

$$
d_{\text {hor }}(x, l)=\min _{y \in l} d_{h o r}(x, y)
$$

Note that for a horizontal line $l=l_{0, b}=\{(x, y): y=b\}$, the horizontal distance between a point $x$ and the line $l$ is either $d_{\text {hor }}(x, l)=0$ if the line passes through $x$ or $d_{\text {hor }}(x, l)=\infty$ else.

Let again $\mathcal{E}=\left\{\left(x_{1}, y_{1}\right), \ldots\left(x_{n}, y_{n}\right)\right\}$ be the set of existing facilities. The problem of locating a line using horizontal distance can be formulated as

$$
\min \sum_{i=1}^{n} d_{h o r}\left(\left(x_{i}, y_{i}\right), l\right)
$$

A horizontal line cannot be optimal for this problem unless all existing facilities are lying on a horizontal line, in other words all existing facilities share the same second coordinate $y_{i}=y$ for all $i \in\{1, \ldots, n\}$. In this special case, the horizontal line $l_{0, y}$ is optimal. Since this case is trivial we will neglect it in the following. Furthermore, we assume that no vertical line is optimal for this problem. Our next step will be to reformulate our problem and it will become clear that this assumption corresponds to the one we already made in Chapter I, namely that there exists no horizontal line which is optimal to the unrestricted vertical problem in the rotated plane. We will see later on that this assumption is not a restriction 


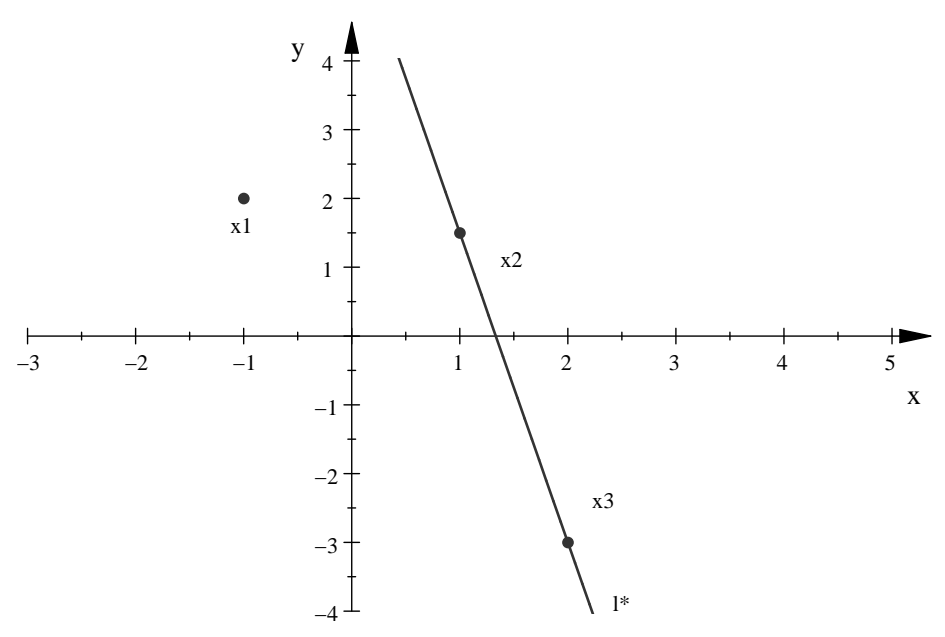

Figure III.1.1. An example with three existing facilities and an optimal solution $l^{*}$ for the unrestricted problem $\left(P_{h o r}\right)$.

since we would be able to find an optimal line that is vertical if there existed one.

As we already mentioned, there is a strong connection between the horizontal and the vertical distance.

Let $\tilde{x}=(x, y) \in \mathbb{R}^{2}$ and let $l_{a, b}$ be a non-vertical-line with slope $a$ and intercept $b$. Assume that we rotate the whole coordinate system counterclockwise by $90^{\circ}$ about the origin. This rotation transforms $(x, y)$ to $(-y, x)$ and $l_{a, b}$ to $l_{-\frac{1}{a},-\frac{b}{a}}$. It is easy to see that the horizontal distance between a point $(x, y)$ and a line $l_{a, b}$ is equivalent to the vertical distance between the corresponding point and the corresponding line in the rotated system. Therefore the following equations hold for the horizontal distance (see also e.g. [Sch99]):

$$
\begin{aligned}
d_{\text {hor }}\left(\tilde{x}, l_{a, b}\right) & =d_{\text {ver }}\left(\left(-x_{2}, x_{1}\right), l_{-\frac{1}{a},-\frac{b}{a}}\right) \\
& =\left|x_{1}-\frac{x_{2}}{a}+\frac{b}{a}\right| \\
& =\left|\frac{1}{a}\left(x_{2}-a x_{1}-b\right)\right| \\
& =\frac{1}{|a|} d_{\text {ver }}\left(\tilde{x}, l_{a, b}\right) .
\end{aligned}
$$


The unrestricted problem of locating a line with horizontal distance can now be reformulated like this:

$$
\begin{aligned}
\min f_{\text {hor }}(a, b) & =\min \sum_{i=1}^{n} d_{\text {hor }}\left(\left(x_{i}, y_{i}\right), l_{a, b}\right) \\
& =\min \frac{1}{|a|} \sum_{i=1}^{n} d_{v e r}\left(\left(x_{i}, y_{i}\right), l_{a, b}\right) \\
& =\min \sum_{i=1}^{n} d_{v e r}\left(\left(-y_{i}, x_{i}\right), l_{-\frac{1}{a},-\frac{b}{a}}\right) \\
& =\min \sum_{i=1}^{n} d_{v e r}\left(\left(-y_{i}, x_{i}\right), l_{\alpha, \beta}\right)
\end{aligned}
$$

with $\alpha=-\frac{1}{a}$ and $\beta=-\frac{b}{a}$.

We will denote the vertical problem in the rotated system corresponding to $\left(P_{\text {hor }}\right)$ by

$$
\min f_{v e r}(\alpha, \beta)=\min \sum_{i=1}^{n} d_{v e r}\left(\left(-y_{i}, x_{i}\right), l_{\alpha, \beta}\right)
$$

and its set of optimal solutions by

$$
\tilde{M}_{v e r}^{\text {opt }}=\left\{\left(\alpha^{*}, \beta^{*}\right): f_{v e r}\left(\alpha^{*}, \beta^{*}\right) \leq f_{v e r}(\alpha, \beta) \forall(\alpha, \beta) \in \mathbb{R}^{2}\right\}
$$

Remember that we made the assumption that no vertical line is optimal to $\left(P_{\text {hor }}\right)$. With the corresponding vertical problem in the rotated system at hand, it is easy to see why this assumption corresponds to the one that there exists no horizontal line which is optimal to the unrestricted vertical problem we made in Chapter [? Assuming that no vertical line is optimal for $\left(P_{h o r}\right)$ is equivalent to assuming that there is no horizontal line that is optimal for $\left(\tilde{P}_{v e r}\right)$ in the rotated system.

We can now treat the unrestricted problem of locating a line with horizontal distance as the unrestricted problem of locating a line with vertical distance in the plane rotated counterclockwise by $90^{\circ}$. We can use the dual space of the rotated plane to find an optimal solution for $\left(\tilde{P}_{v e r}\right)$ and retransform the point first to the 
rotated plane and then back to the unrotated plane where we get an optimal line for $\left(P_{\text {hor }}\right)$. Theorem 1.2 .3 provides us what we need to easily prove the following theorem.

Theorem III.1.1. Let $M_{\text {hor }}^{\text {opt }}:=\left\{\left(a^{*}, b^{*}\right): f_{\text {hor }}\left(a^{*}, b^{*}\right) \leq f_{\text {hor }}(a, b) \forall(a, b) \in \mathbb{R}^{2}\right\}$ and let no vertical line be optimal for $\left(P_{\text {hor }}\right)$. Then either $a^{*}>0$ holds for all $\left(a^{*}, b^{*}\right) \in M_{\text {hor }}^{\text {opt }}$ or $a^{*}<0$ holds for all $\left(a^{*}, b^{*}\right) \in M_{\text {hor }}^{\text {opt }}$.

Proof. We already know that $\left(P_{h o r}\right)$ and $\left(\tilde{P}_{v e r}\right)$ are equivalent with $\alpha=-\frac{1}{a}$ and $\beta=-\frac{b}{a}$. Since there is no optimal line for $\left(P_{h o r}\right)$ that is vertical, there exists no optimal solution to $\left(\tilde{P}_{v e r}\right)$ with $\alpha=0$.

Assume there exist $\left(a^{*}, b^{*}\right),(\hat{a}, \hat{b}) \in M_{h o r}^{\text {opt }}$ satisfying $a^{*}>0$ and $\hat{a}<0$, respectively. This corresponds to $\left(\alpha^{*}, \beta^{*}\right),(\hat{\alpha}, \hat{\beta}) \in \tilde{M}_{v e r}^{\text {opt }}$ satisfying $\alpha^{*}<0$ and $\hat{\alpha}>0$. Since $\left(\tilde{P}_{v e r}\right)$ is a convex problem, it holds that

$$
\lambda(\hat{\alpha}, \hat{\beta})+(1-\lambda)\left(\alpha^{*}, \beta^{*}\right) \in \tilde{M}_{v e r}^{\text {opt }} \forall \lambda \in(0,1) .
$$

Choose $\lambda:=-\frac{\alpha^{*}}{\hat{\alpha}-\alpha^{*}}$. It can easily be shown that $\lambda \in(0,1)$ and therefore $\left(0, \beta^{\prime}\right) \in$ $\tilde{M}_{\text {ver }}^{\text {opt }}$ with $\beta^{\prime}=\lambda \hat{\beta}+(1-\lambda) \beta^{*}$. But this means that there exists a vertical line that is optimal for the problem $\left(P_{h o r}\right)$, which is a contradiction to our assumption.

Up to this point, we know how to handle the horizontal problem. But again, we are interested in the problem of locating a line with horizontal distance when there is a restriction on the slope. This problem can now be formulated as

$$
\begin{aligned}
& \min f_{\text {hor }}(a, b)=\min \sum_{i=1}^{n} d_{h o r}\left(\left(x_{i}, y_{i}\right), l_{a, b}\right) \quad\left(P_{h o r}(s)\right) \\
& \text { s.t. }|a| \leq s
\end{aligned}
$$

where $s \geq 0$.

If we want to reformulate this problem again as a problem with vertical distance, we can look at the rotated coordinate system as we did before. What we get is 
the following problem:

$$
\begin{aligned}
& \min f_{\text {hor }}(a, b)=\min \sum_{i=1}^{n} d_{v e r}\left(\left(-y_{i}, x_{i}\right), l_{-\frac{1}{a},-\frac{b}{a}}\right) \\
& \text { s.t. }|a| \leq s
\end{aligned}
$$

with $s \geq 0$ or equivalently

$$
\begin{array}{ll}
\min f_{\text {ver }}(\alpha, \beta)=\min \sum_{i=1}^{n} d_{\text {ver }}\left(\left(-y_{i}, x_{i}\right), l_{\alpha, \beta}\right) & \\
\text { s.t. }|\alpha| & \geq \tilde{s}
\end{array}
$$

with $\alpha=-\frac{1}{a}, \beta=-\frac{b}{a}$ and $\tilde{s}=\frac{1}{s}$.

Unfortunately, what we get is not longer a convex problem. While the objective function of $\left(\tilde{P}_{v e r}(\tilde{s})\right)$ is again convex, the constraint is not convex any more. Therefore we are not able to apply our results of Section $\llbracket .2$ to solve this problem in contrast to the unrestricted case.

However, we are still able to prove that the solution path of a horizontal problem shares most of the properties that characterize the solution path of a vertical problem. Before we do that, we want to point out that the assumption that there exists no vertical line optimal to $\left(P_{h o r}\right)$ is indeed no restriction.

If we want to solve $\left(P_{h o r}(s)\right)$ for any $s \geq 0$ we start with $s=0$ and let $s$ grow. Therefore it makes sense to start with $\tilde{s}=\infty$ if we want to solve $\left(\tilde{P}_{v e r}(\tilde{s})\right)$ for any $\tilde{s} \geq 0$ since $\tilde{s}=\infty$ corresponds to $s=0$. With growing $s$ we let the corresponding $\tilde{s}$ shrink and solve $\left(\tilde{P}_{v e r}(\tilde{s})\right)$ in order to get solutions for $\left(P_{h o r}(s)\right)$. We do this until we get an optimal solution for the unrestricted problem $\left(P_{h o r}\right)$. But this problem corresponds to $\left(\tilde{P}_{v e r}(0)\right)$ and therefore we would indeed be able to find an optimal solution $\left(\alpha^{*}, \beta^{*}\right)$ with $\alpha^{*}=0$ if there existed one. This type of solution would correspond to a vertical line optimal for $\left(P_{h o r}\right)$ which means that we would be able to find a vertical line optimal to $\left(P_{\text {hor }}\right)$ if there existed one. 


\section{LOCATING LINES WITH A RESTRICTION ON THE SLOPE USING HORIZONTAL DISTANCE

Now we want to show that most of the properties characterizing the solution path of a problem with vertical distance still hold for the solution path of a problem with horizontal distance. We start with the following property that finds its counterpart in the vertical case in Theorem I.2.1.

Theorem III.1.2. There exists a line optimizing $\left(P_{\text {hor }}(s)\right)$ that passes through at least one of the given facilities.

Proof. $\left(P_{\text {hor }}(s)\right)$ is equivalent to $\left(\tilde{P}_{v e r}(\tilde{s})\right)$. If we consider the dual space of the rotated coordinate system, we still have a cell structure given through the lines corresponding to the existing facilities and the two vertical lines corresponding to the given restriction. Even though the constraint is not convex any more, the objective function $f_{v e r}$ is still convex and piecewise linear on each cell. Therefore there exists an optimal solution $\left(\alpha^{*}, \beta^{*}\right)$ that is a vertex of a cell. Such a vertex can be the intersection of two lines corresponding to existing facilities or of a line corresponding to an existing facility and a line corresponding to a restriction. In either case such a vertex corresponds to a line passing through at least one of the existing facilities in the rotated coordinate system. Since $\left(\tilde{P}_{v e r}(\tilde{s})\right)$ and $\left(P_{h o r}(s)\right)$ are equivalent, this gives us a line that is optimal for $\left(P_{h o r}(s)\right)$ in the unrotated coordinate system which still passes through one of the existing facilities.

Next we want to show that all lines optimal for $\left(P_{\text {hor }}(s)\right)$ with $s>0$ are pseudohalving which means that Theorem I.2.2 still holds in the horizontal case excluding $s=0$. We have to exclude the case $s=0$ because all horizontal lines are optimal with an objective function value equal to $\infty$ if not all of the existing facilities are located on a horizontal line. If all the existing facilities are located on a horizontal line, this line is of course optimal and it is obviously pseudo-halving as well. In either case, for $s=0$ one can find optimal solutions that are pseudo-halving but it is not true in general that all optimal lines are pseudo-halving.

In order to prove that Theorem $\underline{I .2 .2}$ holds for $s>0$ we will first prove the following lemma.

Lemma III.1.1. Let $\mathcal{E}=\left\{\left(x_{1}, y_{1}\right), \ldots,\left(x_{n}, y_{n}\right)\right\}$ be the set of existing facilities and let $l_{a, b}$ be a line. Let furthermore $\tilde{\mathcal{E}}=\left\{\left(-y_{1}, x_{1}\right), \ldots,\left(-y_{n}, x_{n}\right)\right\}$ be the set of rotated existing facilities and let $l_{\alpha, \beta}$ be the rotated version of $l_{a, b}$. 
If $l_{a, b}$ is pseudo-halving in the unrotated coordinate system, then $l_{\alpha, \beta}$ is pseudohalving in the rotated coordinate system and vice versa.

Proof. Assume that

$$
y_{i}>a x_{i}+b
$$

for an $\left(x_{i}, y_{i}\right) \in \mathcal{E}$. This means that the point $\left(x_{i}, y_{i}\right)$ lies above the line $l_{a, b}$ in the unrotated plane. This is equivalent to

$$
x_{i}<\alpha\left(-y_{i}\right)+\beta
$$

meaning that the point $\left(-y_{i}, x_{i}\right)$ lies below the corresponding line $l_{\alpha, \beta}$ in the rotated plane. In the same way it can be shown that a point lying below a line in the unrotated plane corresponds to a point lying above the corresponding line in the rotated plane. It therefore holds that

$$
\left|R\left(l_{a, b}\right)\right|=\left|L\left(l_{\alpha, \beta}\right)\right|
$$

and

$$
\left|L\left(l_{a, b}\right)\right|=\left|R\left(l_{\alpha, \beta}\right)\right|
$$

By definition of a pseudo-halving line the lemma follows.

Using the previous lemma we can prove the following theorem.

Theorem III.1.3. A line $l^{*}$ optimal for $\left(P_{\text {hor }}(s)\right)$ with $s>0$ is pseudo-halving.

Proof. Note that in the proof of Theorem $\llbracket .2 .2$ we did not use the fact that $|a| \leq s$. Therefore we can show that any optimal solution to $\left(\tilde{P}_{v e r}(\tilde{s})\right)$ is pseudo-halving using exactly the same proof with the slight difference that we assume $|\alpha| \geq$ $\tilde{s}$. Lemma III.1.1 assures that a pseudo-halving line in the unrotated plane is pseudo-halving if the corresponding line in the rotated plane is pseudo-halving. Since $\left(P_{\text {hor }}(s)\right)$ and $\left(\tilde{P}_{v e r}(\tilde{s})\right)$ are equivalent, any optimal solution to $\left(P_{\text {hor }}(s)\right)$ corresponds to an optimal solution to $\left(\tilde{P}_{v e r}(\tilde{s})\right)$. Since any optimal solution to $\left(\tilde{P}_{v e r}(\tilde{s})\right)$ with $\tilde{s}<\infty$ is pseudo-halving, so is any optimal solution to $\left(P_{\text {hor }}(s)\right)$ with $s>0$. 
Now we know that any optimal solution to our horizontal problem still has the pseudo-halving property if $s>0$ and that we can choose optimal solutions having the pseudo-halving property if $s=0$. In fact, up to now we have not found significant differences between the solution path of the vertical problem and the solution path of the horizontal problem. Unfortunately, this will change if we try to prove a direct analogon of Theorem I.2.4. In order to prove this theorem we used the fact that the vertical problem has a convex constraint. As we already mentioned earlier, the horizontal problem is equivalent to a vertical problem with non-convex constraint. In the horizontal case, it is not longer true that all optimal solutions to $\left(P_{\text {hor }}(s)\right)$ have the same sign in slope as the optimal solutions to $\left(P_{\text {hor }}\right)$ for any $s>0$.

Example III.1.1. Let $\mathcal{E}=\{(0,0),(-3,5),(1,6)\}$ be the set of given facilities. An optimal solution for the unrestricted problem $\left(P_{h o r}\right)$ is $(6,0)$ which means in other words that the line $l_{6,0}$ with slope 6 and intercept 0 is optimal for $\left(P_{\text {hor }}\right)$. Theorem III.1.1 provides that all lines that are optimal for $\left(P_{\text {hor }}\right)$ have positive slope. If we look at the problem $\left(P_{\text {hor }}(1)\right)$ now, it turns out that an optimal solution is $(-1,2)$ or the line $l_{-1,2}$ with slope -1 and intercept 2 , respectively. Therefore an analogon of Theorem I.2.4 cannot hold. In fact it has not even to be true that the slope of all optimal lines of a restricted problem $\left(P_{\text {hor }}(s)\right)$ with a fixed s share the same sign. If we look at $\left(P_{\text {hor }}\left(\frac{1}{2}\right)\right)$ we find out that $\left(-\frac{1}{2}, \frac{7}{2}\right)$ and $\left(\frac{1}{2}, \frac{11}{2}\right)$ are both optimal solutions while they definitely do not share the same sign in slope.

Like the previous example already suggests if you look at it closely, even if we are not able to prove a direct counterpart of Theorem I.2.5, we fortunately are able to prove a theorem that is closely related to Theorem [1.2.5. In order to do so, we first prove the following lemma.

Lemma III.1.2. For any problem $\left(\tilde{P}_{v e r}(\tilde{s})\right)$ with $\tilde{s} \geq \alpha^{*}:=\max \{|\alpha|:(\alpha, \beta) \in$ $\left.\tilde{M}_{\text {ver }}^{\text {opt }}\right\}$ it holds that all optimal solutions $\left(\alpha^{*}(\tilde{s}), \beta^{*}(\tilde{s})\right)$ satisfy $\left|\alpha^{*}(\tilde{s})\right|=\tilde{s}$.

Proof. Without loss of generality let $\alpha \geq 0$ for all $(\alpha, \beta) \in \tilde{M}_{v e r}^{\text {opt }}$ and let $\tilde{s} \geq \alpha^{*}:=$ $\max \left\{|\alpha|:(\alpha, \beta) \in \tilde{M}_{v e r}^{\text {opt }}\right\}$.

Assume there exists a $(\hat{\alpha}, \hat{\beta}) \in \tilde{M}_{\text {ver }}^{\text {opt }}(\tilde{s})$ with $|\hat{\alpha}| \neq \tilde{s}$ meaning that either $\hat{\alpha}>\tilde{s}$ or $\hat{\alpha}<-s$. If $\tilde{s}=\alpha^{*}$ either of these cases is a contradiction to the choice of $\alpha^{*}$. 
Let now be $\tilde{s}>\alpha^{*}$. If $\hat{\alpha}>\tilde{s}$ there exists a $\lambda \in(0,1)$ in such a way that

$$
\lambda \alpha^{*}+(1-\lambda) \hat{\alpha}=\tilde{s}
$$

since we assumed that $\alpha^{*}<\tilde{s}$. We know that $f_{v e r}$ is convex, therefore the following inequalities hold:

$$
\begin{aligned}
f_{v e r}\left(\lambda \alpha^{*}+(1-\lambda) \hat{\alpha}, \lambda \beta^{*}+(1-\lambda) \hat{\beta}\right) & \leq \lambda f_{v e r}\left(\alpha^{*}, \beta^{*}\right)+(1-\lambda) f_{v e r}(\hat{\alpha}, \hat{\beta}) \\
& <\lambda f_{v e r}(\hat{\alpha}, \hat{\beta})+(1-\lambda) f_{v e r}(\hat{\alpha}, \hat{\beta}) \\
& =f_{v e r}(\hat{\alpha}, \hat{\beta}) .
\end{aligned}
$$

This is a contradiction to the optimality of $(\hat{\alpha}, \hat{\beta})$ since $\left(\lambda \alpha^{*}+(1-\lambda) \hat{\alpha}, \lambda \beta^{*}+(1-\right.$ $\lambda) \hat{\beta})$ is feasible for $\left(\tilde{P}_{v e r}(\tilde{s})\right)$.

If $\hat{a}<-\tilde{s}$ there exists a $\lambda \in(0,1)$ in such a way that

$$
\lambda \hat{\alpha}+(1-\lambda) \alpha^{*}=-\tilde{s}
$$

since we assumed that $\alpha^{*}>-\tilde{s}$. Again we have

$$
\begin{aligned}
f_{v e r}\left(\lambda \hat{\alpha}+(1-\lambda) \alpha^{*}, \lambda \hat{\beta}+(1-\lambda) \beta^{*}\right) & \leq \lambda f_{v e r}(\hat{\alpha}, \hat{\beta})+(1-\lambda) f_{v e r}\left(\alpha^{*}, \beta^{*}\right) \\
& <\lambda f_{v e r}(\hat{\alpha}, \hat{\beta})+(1-\lambda) f_{v e r}(\hat{\alpha}, \hat{\beta}) \\
& =f_{v e r}(\hat{\alpha}, \hat{\beta})
\end{aligned}
$$

due to the convexity of $f_{v e r}$. Once more this contradicts the optimality of $(\hat{\alpha}, \hat{\beta})$ since $\left(\lambda \hat{\alpha}+(1-\lambda) \alpha^{*}, \lambda \hat{\beta}+(1-\lambda) \beta^{*}\right)$ is feasible for $\left(\tilde{P}_{v e r}(\tilde{s})\right)$.

This lemma enables us to prove the following theorem.

Theorem III.1.4. For any problem $\left(P_{\text {hor }}(s)\right)$ with $s \leq a^{*}:=\min \{|a|:(a, b) \in$ $M_{\text {hor }}^{\text {opt }}$ it holds that all optimal solutions $\left(a^{*}(s), b^{*}(s)\right)$ satisfy $\left|a^{*}(s)\right|=s$.

Proof. Again we can use the fact that $\left(P_{h o r}(s)\right)$ corresponds to $\left(\tilde{P}_{v e r}(\tilde{s})\right)$. 


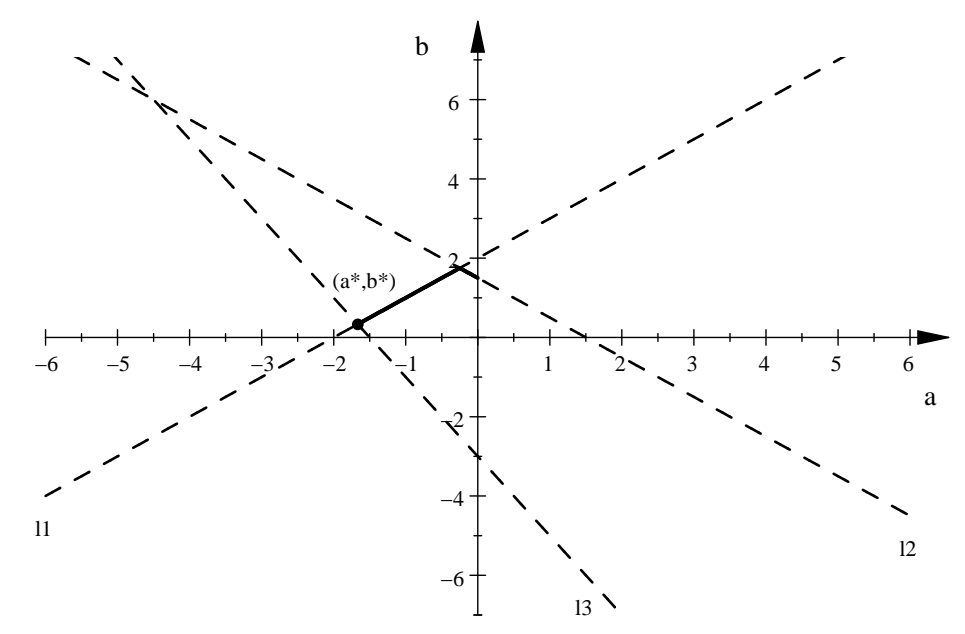

Figure III.1.2. Let $\mathcal{E}=\left\{(-1,2),\left(1, \frac{3}{2}\right),(2,-3)\right\}$. The solid line segments give the solution path for the horizontal case. The path runs along the path of pseudo-halving lines in the negative direction until it ends in the optimal solution $\left(a^{*}, b^{*}\right)$.

Lemma ஹI.1.2 provides that for any $\tilde{s} \geq \alpha^{*}:=\max \left\{|\alpha|:(\alpha, \beta) \in \tilde{M}_{\text {ver }}^{\text {opt }}\right\}$ all optimal solutions $\left(\alpha^{*}(\tilde{s}), \beta^{*}(\tilde{s})\right)$ to $\left(\tilde{P}_{v e r}(\tilde{s})\right)$ satisfy $\left|\alpha^{*}(\tilde{s})\right|=\tilde{s}$.

Since $\tilde{s}=\frac{1}{s}$ and $\alpha=-\frac{1}{a}, \tilde{s} \geq \alpha^{*}$ corresponds to $s \leq a^{*}:=\min \left\{|a|:(a, b) \in M_{\text {hor }}^{\text {opt }}\right\}$. Furthermore we know that any optimal solution $\left(\alpha^{*}(\tilde{s}), \beta^{*}(\tilde{s})\right)$ to $\left(\tilde{P}_{\text {ver }}(\tilde{s})\right)$ corresponds to an optimal solution $\left(a^{*}(s), b^{*}(s)\right)$ to $\left(P_{\text {hor }}(s)\right)$. The fact that all optimal solutions $\left(\alpha^{*}(\tilde{s}), \beta^{*}(\tilde{s})\right)$ to $\left(\tilde{P}_{\text {ver }}(\tilde{s})\right)$ satisfy $\left|\alpha^{*}(\tilde{s})\right|=\tilde{s}$ therefore corresponds to the statement that all optimal solutions to $\left(P_{\text {hor }}(s)\right)$ satisfy $\left|a^{*}(s)\right|=s$. This is exactly what we wanted to show.

The previous theorem assures that all optimal solutions to any restricted horizontal problem that excludes all optimal solutions to the unrestricted problem as well as all optimal solutions to the restricted horizontal problem $\left(P\left(a^{*}\right)\right)$ that allows an optimal solution to the unrestricted problem for the first time have to lie on the boundary of the set of feasible solutions. In contrast to the vertical case we do not know the sign of the slope of an optimal solution for a restricted horizontal problem in the first place as we already noticed in Example III.1.1. This means that the solution path can "jump" from one side of the $b$-axis to the other one and back again at some points that we still have to determine. Figure III.1.2 and Figure III.1.3 show two different types of solution paths for the horizontal problem. In 


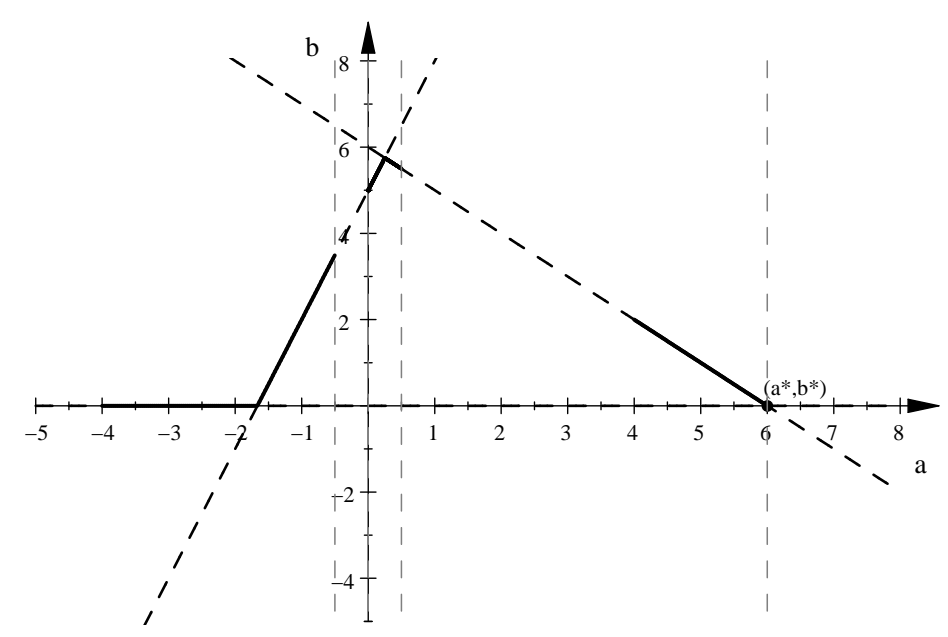

Figure III.1.3. Let $\mathcal{E}=\{(0,0),(-3,5),(1,6)\}$. The solid line segments give the solution path for the horizontal case. There are two values of $s$ where the sign of the slope of optimal solutions of $\left(P_{h o r}(s)\right)$ changes, namely $s=\frac{1}{2}$ and $s=4$.

Figure III.1.2 there are no jumps, the solution path starts at $a=0$ and runs into the direction of the optimal solution $\left(a^{*}, b^{*}\right)=\left(-\frac{5}{3}, \frac{1}{5}\right)$ of the unrestricted problem following the path of pseudo-halving lines. As a matter of fact the solution path of the horizontal problem is identical to the one of the vertical problem (compare Figure I.2.5).

In Figure III.1.3 there are two points at which the solution path jumps from one side of the $b$-axis to the other one. It starts at $a=0$ and runs into the direction of the optimal solution $\left(a^{*}, b^{*}\right)=(6,0)$ until $s=\frac{1}{2}$. Then the path continues from $s=-\frac{1}{2}$ in the negative direction until $s=4$. Now the path jumps again to the other side of the $b$-axis running there until $\left(a^{*}, b^{*}\right)$ is reached. Note that all the time the path follows the path of pseudo-halving lines. We summarize this observations in the following lemma.

Lemma III.1.3. There need not exist a continuous optimal trajectory for the problem $\left(P_{\text {hor }}(s)\right)$.

Proof. Since III.1.3 provides an example for a problem with no continuous optimal trajectory there is nothing left to prove.

Up to now, all we know is that the optimal solutions to $\left(P_{\text {hor }}(s)\right)$ might change their sign in slope at some point, but we do not know where this point actually 
is. Therefore we will show how to find out whether or not the sign changes and if so where it changes in the following. Again we will use $\left(\tilde{P}_{v e r}\right)$ and its dual space. Afterwards we will give examples in order to clarify our approach.

Look at $\left(\tilde{P}_{v e r}\right)$. Without loss of generality assume that all optimal solutions of $\left(\tilde{P}_{v e r}\right)$ have positive slope.

Let $(\hat{\alpha}, \hat{\beta}) \in \tilde{M}_{v e r}^{\text {opt }}$ in such a way that $\hat{\alpha}:=\max \left\{\alpha:(\alpha, \beta) \in \tilde{M}_{\text {ver }}^{\text {opt }}\right\}$. Since $(\hat{\alpha}, \hat{\beta}) \in \tilde{M}_{v e r}^{\text {opt }}$ it holds that $f_{\text {ver }}(\hat{\alpha}, \hat{\beta}) \leq f_{\text {ver }}(-\hat{\alpha}, \bar{\beta})$ for all $\bar{\beta}$ such that $(-\hat{\alpha}, \bar{\beta})$ corresponds to a pseudo-halving line. If the number of existing facilities is even, let $(-\hat{\alpha}, \bar{\beta})$ be the unique point lying on the lower median path at $-\hat{\alpha}$. We know that a solution path of the rotated problem $\left(\tilde{P}_{v e r}\right)$ has to start in $(\hat{\alpha}, \hat{\beta})$.

Let

$$
S:=\left\{(\alpha, \beta):|\alpha| \geq \hat{\alpha},(\alpha, \beta) \text { is the intersection of } l_{-y_{i}, x_{i}} \text { and } l_{-y_{j}, x_{j}}\right\}
$$

with $\left(x_{i}, y_{i}\right) \neq\left(x_{j}, y_{j}\right) \in \mathcal{E}$. Since $\mathcal{E}$ is of finite cardinality $S$ has to be of finite cardinality as well. Assume $S=\left\{\left(\alpha_{1}, \beta_{1}\right), \ldots\left(\alpha_{k}, \beta_{k}\right): k \in \mathbb{N}\right\}$ and assume additionally that $S$ is sorted in such a way that

$$
\left|\alpha_{1}\right|<\left|\alpha_{2}\right|<\ldots<\left|\alpha_{k}\right|
$$

We now want to look at the intervals

$$
\left[\hat{\alpha},\left|\alpha_{1}\right|\right),\left[\left|\alpha_{1}\right|,\left|\alpha_{2}\right|\right), \ldots,\left[\left|\alpha_{k-1}\right|,\left|\alpha_{k}\right|\right),\left[\alpha_{k}, \infty\right)
$$

as well as

$$
\left(-\infty,-\left|\alpha_{k}\right|\right],\left(-\left|\alpha_{k}\right|,-\left|\alpha_{k-1}\right|\right], \ldots,\left(-\left|\alpha_{2}\right|,-\left|\alpha_{1}\right|\right],\left(-\left|\alpha_{1}\right|,-|\hat{\alpha}|\right] .
$$

On each of these intervals no other two lines corresponding to existing facilities intersect. Therefore all points $(\alpha, \beta)$ with $\alpha$ in the same interval lying on the median path or the lower median path if the number of existing facilities is even belong to the same cell. The median path on such an interval can therefore be described by a line $l_{-y, x}$ corresponding to one of the existing facilities. Since we want to know whether the optimal solutions of $\left(\tilde{P}_{v e r}(\tilde{s})\right)$ change signs in slope we 
have to compare the objective function values of $(\tilde{s}, \beta)$ and $\left(-\tilde{s}, \beta^{\prime}\right)$ where $(\tilde{s}, \beta)$ and $\left(-\tilde{s}, \beta^{\prime}\right)$ are points lying on the (lower) median path. If we want to know how the value of the objective function changes with growing $\tilde{s}$, all we have to know is how $\beta$ and $\beta^{\prime}$ change, respectively, since we know that $|\alpha|=\tilde{s}$ for all optimal solutions. Since we can describe the (lower) median path by a line on each interval, we can easily compute how $\beta$ and $\beta^{\prime}$ change with growing $\tilde{s}$ and therefore how the optimal value of the objective function changes on an interval as described below: Remember that we already know that $f_{v e r}(\hat{\alpha}, \hat{\beta}) \leq f_{\text {ver }}(-\hat{\alpha}, \bar{\beta})$. If we now know how the value of the objective function changes on $\left[\hat{\alpha},\left|\alpha_{1}\right|\right)$ and $\left(-\left|\alpha_{1}\right|,-|\hat{\alpha}|\right]$, respectively, we can tell whether the solution path will jump to the other side of the $b$-axis or not: If the value increases faster on the interval $\left(-\left|\alpha_{1}\right|,-|\hat{\alpha}|\right]$ than on the interval $\left[\hat{\alpha},\left|\alpha_{1}\right|\right)$ there cannot be a solution to $\left(\tilde{P}_{\text {ver }}(\tilde{s})\right)$ with $\tilde{s} \in\left(-\left|\alpha_{1}\right|,-|\hat{\alpha}|\right]$. In other words, the solution path will not jump from one side of the $b$-axis to the other one on this interval. If the value increases faster on the interval $\left[\hat{\alpha},\left|\alpha_{1}\right|\right)$ than on the interval $\left(-\left|\alpha_{1}\right|,-|\hat{\alpha}|\right]$ there exist two possibilities: Either the length of the intervals is big enough such that there exists an $\tilde{s} \in\left[\hat{\alpha},\left|\alpha_{1}\right|\right)$ with $f_{v e r}(\tilde{s}, \bar{\beta})=f_{v e r}\left(-\tilde{s}, \bar{\beta}^{\prime}\right)$. This would mean that the solution path jumps to the other side of the $b$-axis. Or the length of the intervals is not big enough such that there exists a $\tilde{s} \in\left[\hat{\alpha},\left|\alpha_{1}\right|\right)$ with $f_{\text {ver }}(\tilde{s}, \bar{\beta})=f_{\text {ver }}\left(-\tilde{s}, \bar{\beta}^{\prime}\right)$. Then again the path would not jump.

Now that we know whether

$$
f_{v e r}\left(\alpha_{1}, \bar{\beta}\right) \leq f_{v e r}\left(-\alpha_{1}, \bar{\beta}^{\prime}\right)
$$

or

$$
f_{v e r}\left(\alpha_{1}, \bar{\beta}\right) \geq f_{v e r}\left(-\alpha_{1}, \bar{\beta}^{\prime}\right)
$$

we can do the same for the intervals $\left[\left|\alpha_{1}\right|,\left|\alpha_{2}\right|\right)$ and $\left(-\left|\alpha_{2}\right|,-\left|\alpha_{1}\right|\right]$ and so on. By doing so, we get all points at which the solution path for the rotated problem jumps from one side of the $\beta$-axis to the other one. Retransforming this to the solution path of the horizontal problem this gives us all points at which the path jumps from one side of the $b$-axis to the other one.

Let us now look at the following examples.

Example III.1.2. Let $\mathcal{E}=\left\{(-1,2),\left(1, \frac{3}{2}\right),(2,-3)\right\}$. Note that these are the same 
existing facilities as in Figure III.1.2, therefore we already know that the solution path of the horizontal problem has no jumps in it. We now want to verify this using the approach as described above.

In the rotated coordinate system we have $\tilde{\mathcal{E}}=\left\{(-2,-1),\left(-\frac{3}{2}, 1\right),(3,2)\right\}$. The optimal solution to the unrestricted problem is given by $\left(\frac{3}{5}, \frac{1}{5}\right)$. The set of intersections $S$ as defined above is

$$
S=\left\{\left(\frac{3}{5}, \frac{1}{5}\right),(4,7)\right\}
$$

Therefore we will look at the intervals

$$
\left[\frac{3}{5}, 4\right),[4, \infty),(-\infty,-4] \text { and }\left(-4,-\frac{3}{5}\right]
$$

We know that $f_{\text {ver }}\left(\frac{3}{5}, \frac{1}{5}\right) \leq f_{\text {ver }}\left(-\frac{3}{5}, \frac{1}{10}\right)$, which means that the solution path starts in the point $\left(\frac{3}{5}, \frac{1}{5}\right)$. Since all intersections have a positive $\alpha$-value the median path corresponding to lines with a negative slope $\alpha$ can be represented by a single line on the whole interval $\left(-\infty,-\frac{3}{5}\right]$, namely $l_{\frac{3}{2}, 1}$. If $\tilde{s}$ grows by $\Delta \tilde{s}$ then $\bar{\beta}$ will therefore grow by $\Delta \tilde{s} \cdot\left(-\frac{3}{2}\right)$. The new point on the median path can therefore be calculated by

$$
\left(\alpha_{\text {new }}, \bar{\beta}_{\text {new }}\right)=\left(-\left(\frac{3}{5}+\Delta \tilde{s}\right), \frac{1}{10}-\Delta \tilde{s} \cdot \frac{3}{2}\right) .
$$

This enables us to calculate how the function value will change on the interval $\left(-\infty,-\frac{3}{5}\right]$ with growing $\tilde{s}$ :

$$
\begin{aligned}
f_{\text {ver }}\left(\alpha_{\text {new }}, \bar{\beta}_{\text {new }}\right)= & f_{\text {ver }}\left(-\left(\frac{3}{5}+\Delta \tilde{s}\right), \frac{1}{10}-\Delta \tilde{s} \cdot \frac{3}{2}\right) \\
= & f_{\text {ver }}\left(-\frac{3}{5}, \frac{1}{10}\right)-\Delta \tilde{s} \cdot\left(\sum_{i \in L} x_{i}-\sum_{i \in R} x_{i}\right) \\
& +\Delta \tilde{s} \cdot\left(-\frac{3}{2}\right) \cdot(|L|-|R|) .
\end{aligned}
$$

In other words, if $\tilde{s}$ grows by $\Delta \tilde{s}$ the function value will grow by 


$$
\begin{aligned}
\frac{\Delta f_{\text {ver }}}{\Delta \tilde{s}} & =\sum_{i \in L} x_{i}-\sum_{i \in R} x_{i} \\
& =-(-2-3)=5
\end{aligned}
$$

since $|L|=|R|=1$.

Now that we know how the value of the objective function behaves on the interval $\left(-\infty,-\frac{3}{5}\right]$ we have to focus on the positive intervals. We will start with the interval $\left[\frac{3}{5}, 4\right)$. On this interval, the median path can be represented by the line $l_{2,-1}$. If $\tilde{s}$ grows by $\Delta \tilde{s}$ then $\bar{\beta}$ will therefore grow by $\Delta \tilde{s} \cdot 2$. The new point on the median path can therefore be calculated by

$$
\left(\alpha_{\text {new }}, \bar{\beta}_{\text {new }}\right)=\left(\frac{3}{5}+\Delta \tilde{s}, \frac{1}{5}+\Delta \tilde{s} \cdot 2\right) .
$$

Again we can compute how the function value will change on this interval with growing $\tilde{s}$ :

$$
\begin{aligned}
f_{\text {ver }}\left(\alpha_{\text {new }}, \bar{\beta}_{\text {new }}\right) & =f_{\text {ver }}\left(\frac{3}{5}+\Delta \tilde{s}, \frac{1}{5}+\Delta \tilde{s} \cdot 2\right) \\
& =f_{\text {ver }}\left(\frac{3}{5}, \frac{1}{5}\right)+\Delta \tilde{s} \cdot\left(\sum_{i \in L} x_{i}-\sum_{i \in R} x_{i}\right)+\Delta \tilde{s} \cdot 2 \cdot(|L|-|R|) .
\end{aligned}
$$

If $\tilde{s}$ grows by $\Delta \tilde{s}$ the function value will grow by

$$
\begin{aligned}
\frac{\Delta f_{v e r}}{\Delta \tilde{s}} & =\sum_{i \in L} x_{i}-\sum_{i \in R} x_{i} \\
& =3-\left(-\frac{3}{2}\right)=\frac{9}{2}
\end{aligned}
$$




\section{LOCATING LINES WITH A RESTRICTION ON THE SLOPE USING HORIZONTAL DISTANCE

But this means that the value of the objective function on the interval $\left(-4,-\frac{3}{5}\right]$ increases faster than on the interval $\left[\frac{3}{5}, 4\right)$, therefore the solution path will not jump to the other side of the b-axis on this interval.

All that is left to do is to look at the interval $[4, \infty)$ and do the same analysis as above. On this interval, the median path can be represented by the line $l_{\frac{3}{2}, 1}$ again. It is easy to see that we start with the point $(4,7)$. The considerations we already did before tell us that

$$
f_{\text {ver }}(4,7) \leq f_{\text {ver }}(-4,-5)
$$

This time $\bar{\beta}$ will grow by $\Delta \tilde{s} \cdot \frac{3}{2}$ if $\tilde{s}$ grows by $\Delta \tilde{s}$. The new point on the median path can now be calculated by

$$
\left(\alpha_{\text {new }}, \bar{\beta}_{\text {new }}\right)=\left(4+\Delta \tilde{s}, 7+\Delta \tilde{s} \cdot \frac{3}{2}\right) .
$$

One more time we can compute how the function value will change on the interval with growing $\tilde{s}$ :

$$
\begin{aligned}
f_{\text {ver }}\left(\alpha_{\text {new }}, \bar{\beta}_{\text {new }}\right) & =f_{\text {ver }}\left(4+\Delta \tilde{s}, 7+\Delta \tilde{s} \cdot \frac{3}{2}\right) \\
& =f_{\text {ver }}(4,7)+\Delta \tilde{s} \cdot\left(\sum_{i \in L} x_{i}-\sum_{i \in R} x_{i}\right)+\Delta \tilde{s} \cdot \frac{3}{2} \cdot(|L|-|R|) .
\end{aligned}
$$

and therefore

$$
\begin{aligned}
\frac{\Delta f_{\text {ver }}}{\Delta \tilde{s}} & =\sum_{i \in L} x_{i}-\sum_{i \in R} x_{i} \\
& =3-(-2)=5 .
\end{aligned}
$$

But this means that the value of the objective function on the interval $(-\infty,-4]$ increases with the same rate as on the interval $[4, \infty)$. Since $f_{\text {ver }}(4,7) \leq f_{\text {ver }}(-4,-5)$ the solution path will again not jump to the other side of the b-axis on this interval. 
Putting these considerations together and retransforming it to the dual space of the unrotated problem we get exactly what we already knew from Figure III.1.2: The solution path of this horizontal problem has no jumps in it. It starts at $s=0$ running into the negative direction until it reaches the optimal solution $\left(-\frac{5}{3}, \frac{1}{3}\right)$.

Example III.1.3. Let now $\mathcal{E}=\{(0,0),(-3,5),(1,6)\}$. Note that these are the same existing facilities as in Figure III.1.3, therefore we already know that the optimal solutions to $\left(P_{\text {hor }}(s)\right)$ will change signs in slope at $s=\frac{1}{2}$ and again at $s=4$. Again we want to verify this using the approach as described above.

In the rotated coordinate system we have $\tilde{\mathcal{E}}=\{(0,0),(-5,-3),(-6,1)\}$. The optimal solution to the unrestricted problem $\left(\tilde{P}_{v e r}\right)$ is given by $\left(-\frac{1}{6}, 0\right)$. The set of intersections $S$ as defined above is

$$
S=\left\{\left(-\frac{1}{6}, 0\right),\left(\frac{3}{5}, 0\right),(-4,-23)\right\}
$$

Therefore we will look at the following intervals:

$$
\left[\frac{1}{6}, \frac{3}{5}\right),\left[\frac{3}{5}, 4\right),[4, \infty)
$$

and

$$
(-\infty,-4],\left(-4,-\frac{3}{5}\right],\left(-\frac{3}{5},-\frac{1}{6}\right]
$$

We know that

$$
f_{\text {ver }}\left(-\frac{1}{6}, 0\right)=\frac{23}{6} \leq \frac{25}{6}=f_{\text {ver }}\left(\frac{1}{6}, 0\right)
$$

which means that the solution path starts in the point $\left(-\frac{1}{6}, 0\right)$. First we look at the intervals $\left[\frac{1}{6}, \frac{3}{5}\right)$ and $\left(-\frac{3}{5},-\frac{1}{6}\right]$. The median path on $\left[\frac{1}{6}, \frac{3}{5}\right)$ can be represented by $l_{0,0}$. Therefore $\bar{\beta}$ stays the same on this interval no matter how $\tilde{s}$ grows. This means that only $\alpha$ changes as $\tilde{s}$ grows, the new point on the median path is

$$
\left(\alpha_{\text {new }}, \bar{\beta}_{\text {new }}\right)=\left(\frac{1}{6}+\Delta \tilde{s}, 0\right) .
$$


As in Example III.1.2, this enables us to calculate how the function value will change on the interval $\left(\frac{1}{6}, \frac{3}{5}\right]$ with growing $\tilde{s}$ :

$$
\begin{aligned}
f_{\text {ver }}\left(\alpha_{\text {new }}, \bar{\beta}_{\text {new }}\right) & =f_{\text {ver }}\left(\frac{1}{6}+\Delta \tilde{s}, 0\right) \\
& =f_{\text {ver }}\left(\frac{1}{6}, 0\right)+\Delta \tilde{s} \cdot\left(\sum_{i \in L} x_{i}-\sum_{i \in R} x_{i}\right) .
\end{aligned}
$$

In other words, if $\tilde{s}$ grows by $\Delta \tilde{s}$ the function value will grow by

$$
\begin{aligned}
\frac{\Delta f_{v e r}}{\Delta \tilde{s}} & =\sum_{i \in L} x_{i}-\sum_{i \in R} x_{i} \\
& =-5-(-6)=1
\end{aligned}
$$

On the interval $\left(-\frac{3}{5},-\frac{1}{6}\right]$, the median path can be represented by $l_{6,1}$. If $\tilde{s}$ grows by $\Delta \tilde{s}$ then $\bar{\beta}$ will therefore grow by $\Delta \tilde{s} \cdot(-6)$.

The new point on the median path can be calculated by

$$
\left(\alpha_{\text {new }}, \bar{\beta}_{\text {new }}\right)=\left(-\left(\frac{1}{6}+\Delta \tilde{s}\right), 0-\Delta \tilde{s} \cdot 6\right)
$$

We compute how the function value will change on this interval with growing $\tilde{s}$ :

$$
\begin{aligned}
f_{\text {ver }}\left(\alpha_{\text {new }}, \bar{\beta}_{\text {new }}\right)= & f_{\text {ver }}\left(-\left(\frac{1}{6}+\Delta \tilde{s}\right), 0-\Delta \tilde{s} \cdot 6\right) \\
= & f_{\text {ver }}\left(-\frac{1}{6}, 0\right)-\Delta \tilde{s} \cdot\left(\sum_{i \in L} x_{i}-\sum_{i \in R} x_{i}\right) \\
& +\Delta \tilde{s} \cdot(-6) \cdot(|L|-|R|),
\end{aligned}
$$

meaning that if $\tilde{s}$ grows by $\Delta \tilde{s}$ the function value will grow by

$$
\begin{aligned}
\frac{\Delta f_{v e r}}{\Delta \tilde{s}} & =\sum_{i \in L} x_{i}-\sum_{i \in R} x_{i} \\
& =-(-5-0)=5 .
\end{aligned}
$$


This shows that the value of the objective function increases much faster on the interval $\left(-\frac{3}{5},-\frac{1}{6}\right]$ than on the interval $\left[\frac{1}{6}, \frac{3}{5}\right)$. We want to find out if there exists an $\tilde{s} \in\left[\frac{1}{6}, \frac{3}{5}\right)$ in such a way that $\left(\tilde{P}_{\text {ver }}(\tilde{s})\right)$ has an optimal solution with positive $\alpha$. To put it in other words, we want to find out if there exists a $\Delta \tilde{s}$ so that $\tilde{s}+\Delta \tilde{s} \in\left[\frac{1}{6}, \frac{3}{5}\right)$ and

$$
\begin{array}{rlrl}
f_{\text {ver }}\left(-\left(\frac{1}{6}+\Delta \tilde{s}\right),-\Delta \tilde{s} \cdot 6\right) & =f_{\text {ver }}\left(\frac{1}{6}+\Delta \tilde{s}, 0\right) \\
& \Leftrightarrow \quad \frac{23}{6}+\Delta \tilde{s} \cdot 5 & =\frac{25}{6}+\Delta \tilde{s} \cdot 1 \\
\Leftrightarrow & \Delta \tilde{s} & =\frac{1}{12} .
\end{array}
$$

Since $\frac{1}{6}+\frac{1}{12}=\frac{1}{4} \in\left[\frac{1}{6}, \frac{3}{5}\right)$ and because of the rates with which the values of the objective function increase on the intervals $\left[\frac{1}{6}, \frac{3}{5}\right)$ and $\left(-\frac{3}{5},-\frac{1}{6}\right]$, respectively, we know that the solution path has to jump from the point $\left(-\frac{1}{4},-\frac{1}{2}\right)$ to the point $\left(\frac{1}{4}, 0\right)$ in order to run in positive direction from there.

Now we know that

$$
f_{\text {ver }}\left(\frac{3}{5}, 0\right)=\frac{23}{5} \leq 6=f_{v e r}\left(-\frac{3}{5},-\frac{13}{5}\right)
$$

which means that the solution path starts for $\tilde{s} \in\left[\frac{3}{5}, 4\right)$ in the point $\left(-\frac{1}{6}, 0\right)$. Next we have to look at the intervals $\left[\frac{3}{5}, 4\right)$ and $\left(-4,-\frac{3}{5}\right]$. The median path on $\left[\frac{3}{5}, 4\right)$ can be represented by $l_{5,-3}$. If $\tilde{s}$ grows by $\Delta \tilde{s}$ then $\bar{\beta}$ will therefore grow by $\Delta \tilde{s} \cdot 5$. The new point on the median path can therefore be calculated by

$$
\left(\alpha_{\text {new }}, \bar{\beta}_{\text {new }}\right)=\left(\frac{3}{5}+\Delta \tilde{s}, 0+\Delta \tilde{s} \cdot 5\right) .
$$

Now we calculate how the function value will change on the interval $\left[\frac{3}{5}, 4\right)$ with growing $\tilde{s}$ : 


$$
\begin{aligned}
f_{\text {ver }}\left(\alpha_{\text {new }}, \bar{\beta}_{\text {new }}\right)= & f_{\text {ver }}\left(\frac{3}{5}+\Delta \tilde{s}, 0+\Delta \tilde{s} \cdot 5\right) \\
= & f_{\text {ver }}\left(\frac{3}{5}, 0\right)+\Delta \tilde{s} \cdot\left(\sum_{i \in L} x_{i}-\sum_{i \in R} x_{i}\right) \\
& +\Delta \tilde{s} \cdot 5 \cdot(|L|-|R|),
\end{aligned}
$$

which means that the function value will grow by

$$
\begin{aligned}
\frac{\Delta f_{v e r}}{\Delta \tilde{s}} & =\sum_{i \in L} x_{i}-\sum_{i \in R} x_{i} \\
& =0-(-6)=6 .
\end{aligned}
$$

if $\tilde{s}$ grows by $\Delta \tilde{s}$. On the interval $\left(-4,-\frac{3}{5}\right]$, the median path can still be represented by $l_{6,1}$. Therefore if $\tilde{s}$ grows by $\Delta \tilde{s}$ the function value will still grow by $\frac{\Delta f_{v e r}}{\Delta \tilde{s}}=5$ as we already computed above.

This shows that the value of the objective function now increases faster on the interval $\left[\frac{3}{5}, 4\right)$ than on the interval $\left(-4,-\frac{3}{5}\right]$. We want to find out if there exists a $\tilde{s} \in\left[\frac{3}{5}, 4\right)$ in such a way that $\left(\tilde{P}_{\text {ver }}(\tilde{s})\right)$ has again an optimal solution with negative $\alpha$ meaning that we would have to jump back to the other side of the $\beta$-axis. Therefore we check whether there exists a $\Delta \tilde{s}$ so that $\tilde{s}+\Delta \tilde{s} \in\left[\frac{3}{5}, 4\right)$ and

$$
\begin{array}{rlrl} 
& & f_{\text {ver }}\left(-\left(\frac{3}{5}+\Delta \tilde{s}\right),-\frac{13}{5}-\Delta \tilde{s} \cdot 6\right) & =f_{\text {ver }}\left(\frac{3}{5}+\Delta \tilde{s}, 0+\Delta \tilde{s} \cdot 5\right) \\
\Leftrightarrow & 6+\Delta \tilde{s} \cdot 5 & =\frac{23}{5}+\Delta \tilde{s} \cdot 6 \\
\Leftrightarrow & \Delta \tilde{s}=\frac{7}{5} .
\end{array}
$$

Since $\frac{3}{5}+\frac{7}{5}=2 \in\left[\frac{3}{5}, 4\right)$ and because of the rates with which the values of the objective function increase on the intervals $\left[\frac{3}{5}, 4\right)$ and $\left(-4,-\frac{3}{5}\right]$, respectively, we know that the solution path has to jump from the point $(2,7)$ to the point $(-2,-11)$ in order to run in negative direction from there. 
III.2. THE RELATIONSHIP BETWEEN LINE LOCATION USING

VERTICAL DISTANCE AND LINE LOCATION USING

This again tells us that

$$
f_{v e r}(-4,-23)=23 \leq 25=f_{v e r}(4,17)
$$

which means that the solution path starts for $\tilde{s} \in[4, \infty)$ in the point $(-4,-23)$. What is left to do is to look at the intervals $[4, \infty)$ and $(-\infty,-4]$. The median path on $[4, \infty)$ can still be represented by $l_{5,-3}$. Therefore if $\tilde{s}$ grows by $\Delta \tilde{s}$ the function value will still grow by $\frac{\Delta f_{v e r}}{\Delta \tilde{s}}=6$ as we already computed above. On the interval $(-\infty,-4]$, the median path can be represented by $l_{5,-3}$ as well. Therefore if $\tilde{s}$ grows by $\Delta \tilde{s}$ the function value will as well grow by $\frac{\Delta f_{v e r}}{\Delta \tilde{s}}=6$ like on the interval $[4, \infty)$. This shows that the value of the objective function on the interval $(-\infty,-4]$ increases with the same rate as on the interval $[4, \infty)$. Since $f_{\text {ver }}(-4,-23) \leq$ $f_{\text {ver }}(4,17)$ there are no more "jumps" in the solution path.

Retransforming these considerations to the solution path in the dual space of the unrotated problem $\left(P_{\text {hor }}\right)$, we know that the solution path starts at $s=0$ running in the positive direction until $s=\frac{1}{2}$. There it jumps from the point $\left(\frac{1}{2}, \frac{11}{2}\right)$ to the point $\left(-\frac{1}{2}, \frac{7}{2}\right)$ in order to continue in the negative direction. It keeps running in negative direction until $s=4$, then again it jumps from the point $(-4,0)$ to the point $(4,2)$. From there it keeps running in positive direction until it reaches the optimal solution $(6,0)$.

If we take a look at Figure III.1.3 we will notice that these results again correspond exactly to the solution path shown there.

\section{III.2 The relationship between line location us- ing vertical distance and line location using horizontal distance}

As we have seen, there is a strong connection between the vertical and the horizontal distance. Let again $\mathcal{E}=\left\{\left(x_{1}, y_{1}\right), \ldots,\left(x_{n}, y_{n}\right)\right\}$ be the set of existing facilities. Assume we want to locate a line with vertical distance and another one with horizontal distance. In other words, we are searching for optimal solutions to $\left(P_{v e r}(s)\right)$ and $\left(P_{\text {hor }}(s)\right)$, respectively. Is there any connection between the solutions of these 


\section{LOCATING LINES WITH A RESTRICTION ON THE SLOPE USING HORIZONTAL DISTANCE

two problems?

If we want to find an answer to this question one way to do so is to compare the optimal trajectories of these problems. Fortunately, both trajectories run in the same dual space, therefore it is convenient to compare them. Both solution paths start with a horizontal line and then the absolute value of the slope increases until we reach an optimal solution for $\left(P_{v e r}\right)$ or $\left(P_{h o r}\right)$, respectively.

In this section we want to conclude some statements about the relationship of line location problems with vertical and horizontal distances and a restriction on the slope. For the rest of this section, we assume without loss of generality that there exists no horizontal line which is optimal to $\left(P_{v e r}\right)$ as well as that there exists no vertical line which is optimal to $\left(P_{h o r}\right)$.

The first idea one might have is the following: Theorem I.2.3 states that all lines optimal for $\left(P_{v e r}\right)$ share the same sign in slope meaning that either all lines optimal to $\left(P_{v e r}\right)$ have positive slope or they all have negative slope. Theorem III.1.1 states the same for all lines optimal for $\left(P_{h o r}\right)$. It would be a nice property if all lines optimal for $\left(P_{v e r}\right)$ and all lines optimal for $\left(P_{h o r}\right)$ shared the same sign in slope. For the optimal trajectories that would mean that an optimal trajectory of the vertical problem and one of the horizontal problem would end in the same half-space of the dual space no matter how the trajectories behave if $s \in(0,|\hat{a}|)$ with $(\hat{a}, \hat{b}) \in M_{h o r}^{\text {opt }}$. In other words, even if the trajectory of the horizontal problem might jump from one side of the $b$-axis to the other one in the end it would end up on the same side as the trajectory of the vertical problem.

Unfortunately, this is not true in general. The optimal lines of $\left(P_{v e r}\right)$ and the ones of $\left(P_{h o r}\right)$ do not have to share the same sign in slope as Figure III.2.1 shows. The solid line is optimal for $\left(P_{v e r}\right)$ with existing facilities $\mathcal{E}=\{(-0.25,-3),(1,0)$, $(-0.5,1),(0.5,4)\}$. The dashed line is optimal for $\left(P_{\text {hor }}\right)$ with the same existing facilities. Obviously they do not share the same sign in slope.

But even if we cannot say whether the slopes of the optimal solutions of $\left(P_{v e r}\right)$ and $\left(P_{h o r}\right)$ share the same sign or not, we can make a statement about their absolute values. A similar statement can be found in [MN80].

Lemma III.2.1. If $(\hat{a}, \hat{b}) \in M_{\text {hor }}^{\text {opt }}$ and $\left(a^{*}, b^{*}\right) \in M_{\text {ver }}^{\text {opt }}$ then $|\hat{a}| \geq\left|a^{*}\right|$. 
III.2. THE RELATIONSHIP BETWEEN LINE LOCATION USING

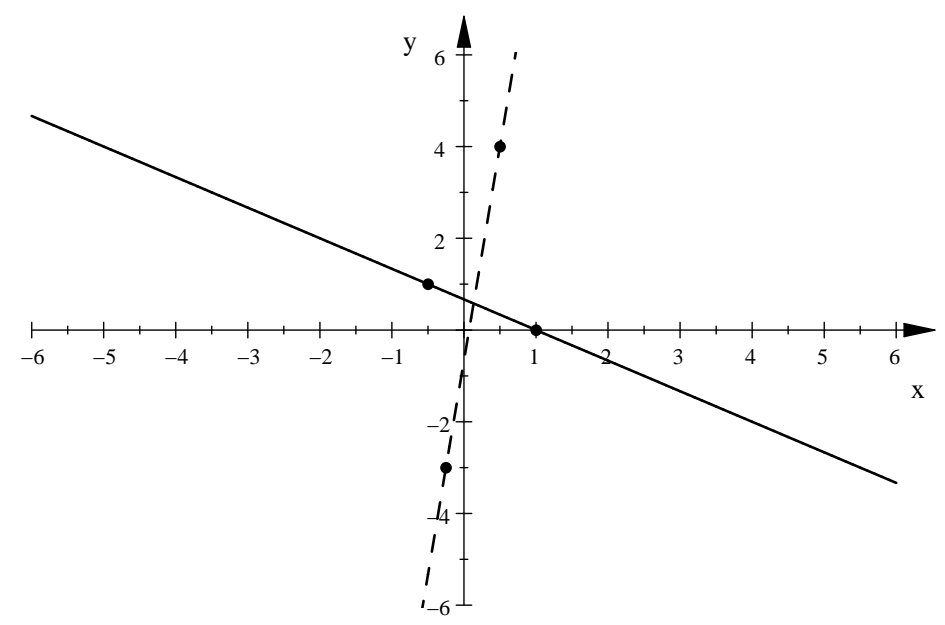

Figure III.2.1. Let $\mathcal{E}=\{(-0.25,-3),(1,0),(-0.5,1),(0.5,4)\}$. The solid line is optimal for $\left(P_{v e r}\right)$ while the dashed one is optimal for $\left(P_{h o r}\right)$.

Proof. Assume $\left|a^{*}\right|>|\hat{a}|$. Then

$$
\begin{aligned}
f_{\text {hor }}(\hat{a}, \hat{b}) & =\frac{1}{|\hat{a}|} \sum_{i=1}^{n} d_{\text {ver }}\left(\left(x_{i}, y_{i}\right), l_{\hat{a}, \hat{b}}\right) \\
& \geq \frac{1}{|\hat{a}|} \sum_{i=1}^{n} d_{v e r}\left(\left(x_{i}, y_{i}\right), l_{a^{*}, b^{*}}\right) \\
& >\frac{1}{\left|a^{*}\right|} \sum_{i=1}^{n} d_{v e r}\left(\left(x_{i}, y_{i}\right), l_{a^{*}, b^{*}}\right) \\
& =f_{\text {hor }}\left(a^{*}, b^{*}\right)
\end{aligned}
$$

which is a contradiction to $(\hat{a}, \hat{b}) \in M_{h o r}^{o p t}$.

We will use this lemma for some of the proofs in the next section. Now we want to prove a theorem that will help us to understand the horizontal trajectory better. This theorem establishes a nice connection between the two optimal trajectories.

Theorem III.2.1. Let $\mathcal{E}=\left\{\left(x_{1}, y_{1}\right), \ldots,\left(x_{n}, y_{n}\right)\right\}$ be the set of existing facilities and let $\left(a^{*}, b^{*}\right) \in M_{\text {ver }}^{\text {opt }}$ in such a way that $\left|a^{*}\right|=\min \left\{|a|:(a, b) \in M_{\text {ver }}^{\text {opt }}\right\}$. If $s \in\left(0,\left|a^{*}\right|\right]$ the optimal solutions to $\left(P_{\text {ver }}(s)\right)$ and $\left(P_{\text {hor }}(s)\right)$ share the same sign in slope. 
Proof. Without loss of generality we assume that all optimal solutions of $\left(P_{\text {ver }}\right)$ have positive slope. Theorem 1.2 .4 states that then all optimal solutions of $\left(P_{v e r}(s)\right)$ have positive slope for all $s \geq 0$ and therefore for all $s \in\left(0, a^{*}\right]$ in particular.

Let $s^{\prime} \in\left(0, a^{*}\right]$ and assume that there exists an optimal solution to $\left(P_{\text {hor }}\left(s^{\prime}\right)\right)$ having negative slope, that means it exists a $\left(a^{\prime}, b^{\prime}\right) \in M_{h o r}^{\text {opt }}\left(s^{\prime}\right)$ with $a^{\prime}<0$. Since $\left(a^{\prime}, b^{\prime}\right)$ is optimal it holds that

$$
f_{\text {hor }}\left(a^{\prime}, b^{\prime}\right) \leq f_{\text {hor }}(a, b) \forall(a, b):|a| \leq s^{\prime}
$$

Let $(\tilde{a}, \tilde{b})$ be an optimal solution to $\left(P_{v e r}\left(s^{\prime}\right)\right)$. By our assumption and by definition of $\left(P_{v e r}\left(s^{\prime}\right)\right)$ it holds that $\tilde{a} \geq 0$ and $\tilde{a} \leq s^{\prime}$. Therefore it must hold that

$$
f_{\text {hor }}\left(a^{\prime}, b^{\prime}\right) \leq f_{\text {hor }}(\tilde{a}, \tilde{b}) \text {. }
$$

Theorem I.2.5 and Theorem $\amalg$ III.1.4 provide that $\tilde{a}$ and $a^{\prime}$ satisfy $\tilde{a}=s^{\prime}$ and $\left|a^{\prime}\right|=s^{\prime}$, respectively, because $s^{\prime} \leq a^{*} \leq \min \left\{|a|:(a, b) \in M_{h o r}^{\text {opt }}\right\}$ due to Lemma III.2.1. But we already know from Section III.1 that then it holds that

$$
\begin{array}{rlrl}
f_{\text {hor }}\left(a^{\prime}, b^{\prime}\right) & \leq f_{\text {hor }}(\tilde{a}, \tilde{b}) \\
\Rightarrow & & \frac{1}{\left|a^{\prime}\right|} f_{\text {ver }}\left(a^{\prime}, b^{\prime}\right) & \leq \frac{1}{|\tilde{a}|} f_{\text {ver }}(\tilde{a}, \tilde{b}) \\
\Rightarrow & \frac{1}{s^{\prime}} f_{\text {ver }}\left(a^{\prime}, b^{\prime}\right) & \leq \frac{1}{s^{\prime}} f_{\text {ver }}(\tilde{a}, \tilde{b}) \\
\Rightarrow & f_{\text {ver }}\left(a^{\prime}, b^{\prime}\right) & \leq f_{\text {ver }}(\tilde{a}, \tilde{b}) .
\end{array}
$$

Therefore $\left(a^{\prime}, b^{\prime}\right)$ is optimal for $\left(P_{\text {ver }}\left(s^{\prime}\right)\right)$ as well which is a contradiction to Theorem I.2.5, Our theorem follows.

In fact, the previous theorem tells us more about optimal trajectories. Not only that all optimal solutions to $\left(P_{\text {ver }}(s)\right)$ and $\left(P_{\text {hor }}(s)\right)$ share the same sign in slope as long as $s \in\left(0, a^{*}\right]$, in fact we can find optimal trajectories that are identical.

Theorem III.2.2. We can find optimal trajectories of $\left(P_{\text {ver }}(s)\right)$ and $\left(P_{\text {hor }}(s)\right)$ such that they are identical on the interval $\left[0, a^{*}\right]$.

Proof. Let us choose the optimal solutions of $\left(P_{\text {hor }}(0)\right)$ that are pseudo-halving as a 


\section{III.2. THE RELATIONSHIP BETWEEN LINE LOCATION USING VERTICAL DISTANCE AND LINE LOCATION USING 74 HORIZONTAL DISTANCE}

start of the trajectory of $\left(P_{h o r}(s)\right)$ what we can do as we mentioned in Section $\amalg$ II.1. Then all optimal solutions to $\left(P_{\text {ver }}(s)\right)$ as well as to $\left(P_{\text {hor }}(s)\right)$ have to be pseudohalving (see Theorem I.2.2 and Theorem III.1.3) or at least are pseudo-halving by choice in the case of $s=0$. Moreover, all optimal solutions to $\left(P_{\text {ver }}(s)\right)$ and $\left(P_{\text {hor }}(s)\right)$ have to lie on the boundary of the set of feasible solutions (see Theorem I.2.5 and Theorem (II.1.4). Together with Theorem III.2.1 that means that the we can find optimal trajectories of the vertical and the horizontal problem that are in fact identical on the interval $\left[0, a^{*}\right]$.

This is what you can observe in Figure II.2.2 and Figure III.2.3. There you can see optimal trajectories of the vertical and the horizontal problem with given facilities $\mathcal{E}=\{(-0.25,-3),(1,0),(-0.5,1),(0.5,4)\}$. We already looked at optimal lines for the corresponding unrestricted problems in Figure III.2.1 therefore we already know that the optimal solutions do not share the same sign in slope. The optimal solution for $\left(P_{v e r}\right)$ is $\left(-\frac{2}{3}, \frac{2}{3}\right)$. You can observe that the trajectories are indeed identical for $s \in\left[0, \frac{2}{3}\right]$. The trajectory of the horizontal problem does not jump to the other side of the $b$-axis until $s>\frac{2}{3}$. Since optimal trajectories of the vertical and the horizontal problem are identical for $s \in\left[0, a^{*}\right]$ we can of course apply Theorem I.2.6 in order to find out in which direction the horizontal path will start to run. Unfortunately this does not help us too much because we still have to examine the trajectory for jumps. 


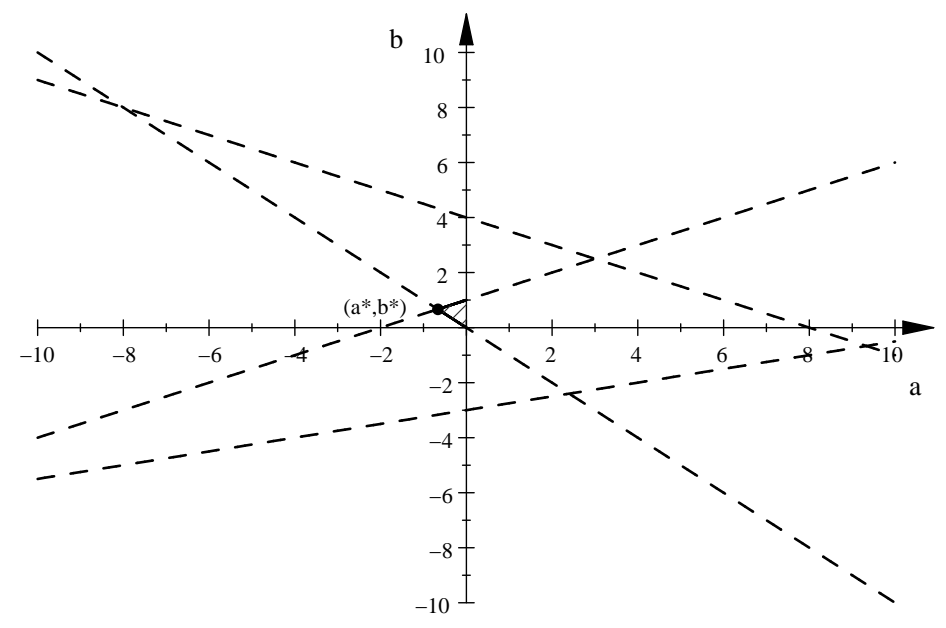

Figure III.2.2. The shaded area is the solution path of $\left(P_{v e r}\right)$ with given facilities $\mathcal{E}=\{(-0.25,-3),(1,0),(-0.5,1),(0.5,4)\}$ in the dual space. It runs in the negative direction until it reaches the optimal solution $\left(a^{*}, b^{*}\right)=\left(-\frac{2}{3}, \frac{2}{3}\right)$

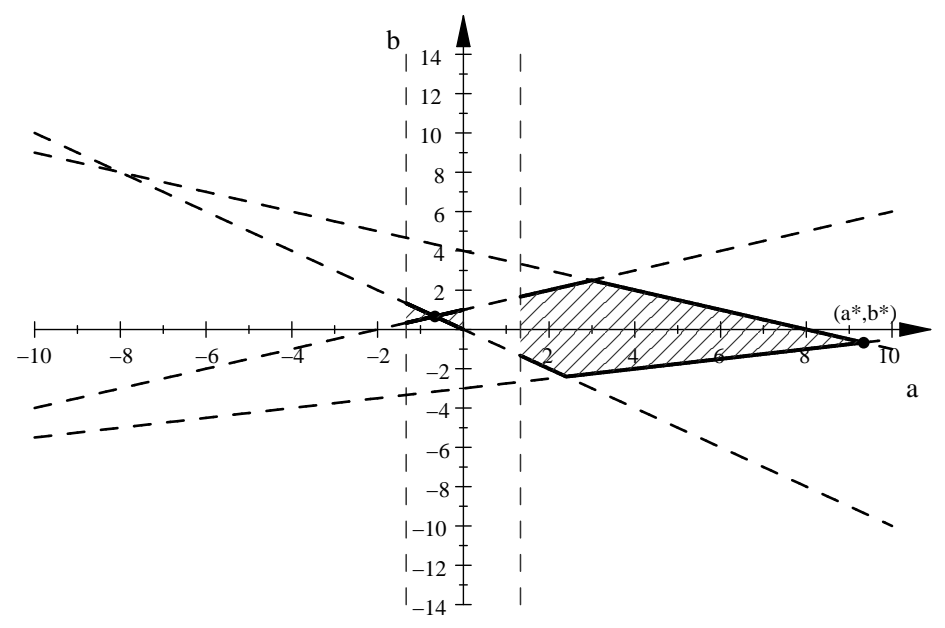

Figure III.2.3. The shaded area is the solution path of $\left(P_{h o r}\right)$ with given facilities $\mathcal{E}=\{(-0.25,-3),(1,0),(-0.5,1),(0.5,4)\}$ in the dual space. It is identical to the solution path of $\left(P_{v e r}\right)$ for $s \in\left[0, \frac{2}{3}\right]$. From the optimal solution $\left(-\frac{2}{3}, \frac{2}{3}\right)$ for $\left(P_{v e r}\right)$ it continues to run in the negative direction until $s=-\frac{4}{3}$. There it jumps to the positive half-space and starts running in the positive direction until it reaches the optimal solution $\left(a^{*}, b^{*}\right)=\left(\frac{28}{3},-\frac{2}{3}\right)$ for $\left(P_{h o r}\right)$. 



\section{Chapter IV}

\section{Locating lines with a restriction on the slope using $l_{1}$-distance}

In this chapter we will consider the problem of locating a line with a restriction on the slope using yet another distance, namely the rectangular distance $l_{1}$. We will introduce this distance and the formulation of our problem in the following. After doing so, we will point out how this distance is connected to the vertical as well as to the horizontal distance and how we can make statements about the solution path for this new problem using the results we already have for the vertical and the horizontal problem. In order to do so, we will assume that there is no horizontal line optimal to $\left(P_{v e r}\right)$ and no vertical line optimal to $\left(P_{h o r}\right)$ as we did in Chapter [I and Chapter III, respectively, throughout the whole chapter. Even if it seems straight-forward at a first glance, there has some work to be done before we can benefit from our previous results. Theorem I.2.5 as well as Theorem III.1.4 will again play an important role in this chapter.

\section{IV.1 Line location with rectangular distance}

We first give a formula for the rectangular distance between a point $(x, y) \in \mathbb{R}^{2}$ and a non-vertical line $l_{a, b}$. This formula is well-known and can be found for example in [MN83], [MT83] or [Sch99]. 
Lemma IV.1.1. Let $l_{a, b}$ be a non-vertical line and $(x, y) \in \mathbb{R}^{2}$. Then the rectangular distance between this point and the line is given by

$$
\begin{aligned}
l_{1}\left((x, y), l_{a, b}\right) & =\min \left\{|x a-y+b|, \frac{1}{|a|}|x a-y+b|\right\} \\
& =\min \left\{d_{\text {ver }}\left((x, y), l_{a, b}\right), \frac{1}{|a|} d_{v e r}\left((x, y), l_{a, b}\right)\right\} \\
& =\min \left\{d_{\text {ver }}\left((x, y), l_{a, b}\right), d_{\text {hor }}\left((x, y), l_{a, b}\right)\right\} .
\end{aligned}
$$

The following inequalities hold (see e.g. [Sch99]):

$$
l_{1}\left(\left(x_{i}, y_{i}\right), l_{a, b}\right)=\left\{\begin{array}{l}
d_{v e r}\left(\left(x_{i}, y_{i}\right), l_{a, b}\right) \text { if }|a|<1 \\
d_{\text {hor }}\left(\left(x_{i}, y_{i}\right), l_{a, b}\right) \text { if }|a|>1 \\
d_{\text {ver }}\left(\left(x_{i}, y_{i}\right), l_{a, b}\right)=d_{h o r}\left(\left(x_{i}, y_{i}\right), l_{a, b}\right) \text { if }|a|=1 .
\end{array}\right.
$$

The unrestricted problem of locating a non-vertical line with rectangular distance has the form

$$
\min \sum_{i=1}^{n} l_{1}\left(\left(x_{i}, y_{i}\right), l_{a, b}\right)
$$

To solve this problem, it is enough to determine a line optimal for $\left(P_{v e r}\right)$ and one that is optimal to $\left(P_{\text {hor }}\right)$ and to choose the line with smaller objective function value. Since we already know that for both problems $\left(P_{v e r}\right)$ and $\left(P_{h o r}\right)$ there exists an optimal line that passes through at least two existing facilities and that all optimal lines are pseudo-halving, this also holds for an optimal solution to $\left(P_{l_{1}}\right)$.

Lemma IV.1.2. ([Sha78], [MT83], [MN83], [Sch99])

For the rectangular distance $l_{1}$ the following criteria hold.

a) There exists a line optimal for $\left(P_{l_{1}}\right)$ passing through two of the existing facilities.

b) All lines optimal for $\left(P_{l_{1}}\right)$ are pseudo-halving.

If we deal with the rectangular problem we have to consider three different cases. Let $\left(a^{*}, b^{*}\right) \in M_{\text {ver }}^{\text {opt }}$ with $a^{*}=\min \left\{|a|:(a, b) \in M_{\text {ver }}^{\text {opt }}\right\}$ and $(\hat{a}, \hat{b}) \in M_{\text {hor }}^{\text {opt }}$ with 
$\hat{a}=\min \left\{|a|:(a, b) \in M_{h o r}^{\text {opt }}\right\}$, respectively. The three different cases that can occur are the following:

- $\left|a^{*}\right| \leq 1,|\hat{a}| \leq 1$,

- $\left|a^{*}\right|>1,|\hat{a}|>1$,

- $\left|a^{*}\right| \leq 1,|\hat{a}|>1$.

The case $\left|a^{*}\right|>1$ while $|\hat{a}| \leq 1$ cannot occur due to Lemma III.2.1 which states that $\left|a^{*}\right| \leq|\hat{a}|$. We want to record some observations for the different cases.

Lemma IV.1.3. a) If $\left|a^{*}\right| \leq 1$ and $|\hat{a}| \leq 1$ then $f_{\text {ver }}\left(a^{*}, b^{*}\right) \leq f_{\text {hor }}(\hat{a}, \hat{b})$.

b) If $\left|a^{*}\right|>1$ and $|\hat{a}|>1$ then $f_{\text {ver }}\left(a^{*}, b^{*}\right)>f_{\text {hor }}(\hat{a}, \hat{b})$.

Proof. a) Since $\left(a^{*}, b^{*}\right) \in M_{v e r}^{o p t}$ and $|\hat{a}| \leq 1$ the first part of the lemma follows:

$$
\begin{aligned}
f_{\text {ver }}\left(a^{*}, b^{*}\right) & \leq f_{\text {ver }}(\hat{a}, \hat{b}) \\
& \leq \frac{1}{|\hat{a}|} f_{\text {ver }}(\hat{a}, \hat{b}) \\
& =f_{\text {hor }}(\hat{a}, \hat{b}) .
\end{aligned}
$$

b) Since $(\hat{a}, \hat{b}) \in M_{h o r}^{o p t}$ and $\left|a^{*}\right|>1$ the second part of the lemma follows:

$$
\begin{aligned}
f_{\text {ver }}\left(a^{*}, b^{*}\right) & >\frac{1}{\left|a^{*}\right|} f_{\text {ver }}\left(a^{*}, b^{*}\right) \\
& =f_{\text {hor }}\left(a^{*}, b^{*}\right) \\
& \geq f_{\text {hor }}(\hat{a}, \hat{b})
\end{aligned}
$$

For the case $\left|a^{*}\right| \leq 1$ while $|\hat{a}|>1$ we cannot make any proposition: It might happen that $f_{v e r}\left(a^{*}, b^{*}\right) \leq f_{\text {hor }}(\hat{a}, \hat{b})$ but it might as well happen that $f_{\text {ver }}\left(a^{*}, b^{*}\right) \geq$ $f_{\text {hor }}(\hat{a}, \hat{b})$. 
Of course there is a strong connection between the sign in slope of optimal solutions to $\left(P_{l_{1}}\right)$ and the sign in slope of optimal solutions to $\left(P_{v e r}\right)$ or $\left(P_{h o r}\right)$ due to the formula of the rectangular distance. This connection depends strongly on the three cases that we mentioned above. We conclude the following lemma.

Lemma IV.1.4. Let $\mathcal{E}=\left\{\left(x_{1}, y_{1}\right), \ldots,\left(x_{n}, y_{n}\right)\right\}$ be the set of existing facilities. Furthermore, let $M_{l_{1}}^{\text {opt }}, M_{\text {ver }}^{\text {opt }}$ and $M_{\text {hor }}^{\text {opt }}$ be the sets of optimal solutions to $\left(P_{l_{1}}\right)$, $\left(P_{v e r}\right)$ and $\left(P_{\text {hor }}\right)$, respectively. Depending on two of the different cases mentioned above the following equivalences hold.

If $\left|a^{*}\right| \leq 1$ and $|\hat{a}| \leq 1$ then $a>0$ for all $(a, b) \in M_{l_{1}}^{\text {opt }} \Leftrightarrow a>0$ for all $(a, b) \in M_{\text {ver }}^{\text {opt }}$. Analogously $a<0$ for all $(a, b) \in M_{l_{1}}^{\text {opt }} \Leftrightarrow a<0$ for all $(a, b) \in M_{\text {ver }}^{\text {opt }}$.

If $\left|a^{*}\right|>1$ and $|\hat{a}|>1$ then $a>0$ for all $(a, b) \in M_{l_{1}}^{\text {opt }} \Leftrightarrow a>0$ for all $(a, b) \in M_{\text {hor }}^{\text {opt }}$. Analogously $a<0$ for all $(a, b) \in M_{l_{1}}^{\text {opt }} \Leftrightarrow a<0$ for all $(a, b) \in M_{\text {hor }}^{\text {opt }}$.

Proof. We only prove the statements for $a>0$.

We start with the case $\left|a^{*}\right| \leq 1$ and $|\hat{a}| \leq 1$.

" $\Rightarrow$ " Assume $a>0$ for all $(a, b,) \in M_{l_{1}}^{\text {opt }}$. Since

$$
l_{1}\left(\left(x_{i}, y_{i}\right), l_{a, b}\right)=\min \left\{d_{v e r}\left(\left(x_{i}, y_{i}\right), l_{a, b}\right), d_{h o r}\left(\left(x_{i}, y_{i}\right), l_{a, b}\right)\right\}
$$

for any $(a, b) \in M_{l_{1}}^{\text {opt }}$ it is either $(a, b) \in M_{v e r}^{\text {opt }}$ or $(a, b) \in M_{h o r}^{\text {opt }}$. We know that $\left|a^{*}\right| \leq 1$ and $|\hat{a}| \leq 1$. Therefore we know that $f_{l_{1}}(a, b)=f_{\text {ver }}(a, b)$ in either case. But then $(a, b) \in M_{v e r}^{o p t}$ has to hold. Due to our assumption that there is no horizontal line optimal for $\left(P_{v e r}\right)$ this means that $a>0$ for all $(a, b) \in M_{\text {ver }}^{\text {opt }}$ due to Theorem I.2.3.

$" \Leftarrow "$ Assume $a>0$ for all $(a, b) \in M_{v e r}^{o p t}$. Lemma IV.1.3 tells us that $f_{v e r}\left(a^{*}, b^{*}\right) \leq$ $f_{\text {hor }}(\hat{a}, \hat{b})$. Therefore and because of

$$
l_{1}\left(\left(x_{i}, y_{i}\right), l_{a, b}\right)=\min \left\{d_{v e r}\left(\left(x_{i}, y_{i}\right), l_{a, b}\right), d_{h o r}\left(\left(x_{i}, y_{i}\right), l_{a, b}\right)\right.
$$

if $\left(a^{\prime}, b^{\prime}\right) \in M_{l_{1}}^{\text {opt }}$ then $\left(a^{\prime}, b^{\prime}\right) \in M_{v e r}^{\text {opt }}$ must hold. By assumption it follows that $a^{\prime}>0$.

Now we look at the case $\left|a^{*}\right|>1$ and $|\hat{a}|>1$. 
" $\Rightarrow$ " Assume $a>0$ for all $(a, b,) \in M_{l_{1}}^{\text {opt }}$. Since

$$
l_{1}\left(\left(x_{i}, y_{i}\right), l_{a, b}\right)=\min \left\{d_{v e r}\left(\left(x_{i}, y_{i}\right), l_{a, b}\right), d_{h o r}\left(\left(x_{i}, y_{i}\right), l_{a, b}\right)\right\}
$$

for any $(a, b) \in M_{l_{1}}^{\text {opt }}$ it is either $(a, b) \in M_{\text {ver }}^{\text {opt }}$ or $(a, b) \in M_{h o r}^{\text {opt }}$. We know that $\left|a^{*}\right|>1$ and $|\hat{a}|>1$. Therefore we know that $f_{l_{1}}(a, b)=f_{\text {hor }}(a, b)$ in either case. But then $(a, b) \in M_{h o r}^{\text {opt }}$ has to hold. Due to our assumption that there is no vertical line optimal for $\left(P_{\text {hor }}\right)$ this means that $a>0$ for all $(a, b) \in M_{h o r}^{\text {opt }}$ due to Theorem II.1.1.

$" \Leftarrow "$ Assume $a>0$ for all $(a, b) \in M_{\text {hor }}^{\text {opt }}$ Lemma IV.1.3 tells us that $f_{\text {ver }}\left(a^{*}, b^{*}\right) \geq$ $f_{\text {hor }}(\hat{a}, \hat{b})$. Therefore and because of

$$
l_{1}\left(\left(x_{i}, y_{i}\right), l_{a, b}\right)=\min \left\{d_{v e r}\left(\left(x_{i}, y_{i}\right), l_{a, b}\right), d_{h o r}\left(\left(x_{i}, y_{i}\right), l_{a, b}\right)\right.
$$

if $\left(a^{\prime}, b^{\prime}\right) \in M_{l_{1}}^{\text {opt }}$ then $\left(a^{\prime}, b^{\prime}\right) \in M_{h o r}^{\text {opt }}$ must hold. By assumption it follows that $a^{\prime}>0$.

Again we cannot make a similar proposition in the third case. What we of course know is that either $(a, b) \in M_{\text {ver }}^{\text {opt }}$ for all $(a, b) \in M_{l_{1}}^{\text {opt }}$ or $(a, b) \in M_{h o r}^{\text {opt }}$ for all $(a, b) \in$ $M_{l_{1}}^{\text {opt }}$ depending on whether $f_{\text {ver }}\left(a^{*}, b^{*}\right) \leq f_{\text {hor }}(\hat{a}, \hat{b})$ or $f_{\text {ver }}\left(a^{*}, b^{*}\right) \geq f_{\text {hor }}(\hat{a}, \hat{b})$. Therefore even in the third case we get again that all optimal solutions to $\left(P_{l_{1}}\right)$ share the same sign in slope. We just cannot say which sign it will be. We want to finish this section with the following proposition.

Proposition IV.1.1. The following holds:

If $a^{*} \neq 0$ for all $\left(a^{*}, b^{*}\right) \in M_{l_{1}}^{\text {opt }}$ then either $a^{*}>0$ for all $\left(a^{*}, b^{*}\right) \in M_{l_{1}}^{\text {opt }}$ or $a^{*}<0$ for all $\left(a^{*}, b^{*}\right) \in M_{l_{1}}^{\text {opt }}$.

Proof. This is a direct consequence of Theorem I.2.3 and Lemma IV.1.4 as well as of our remark following Lemma IV.1.4. 


\section{IV.2 Line location with rectangular distance and a restriction on the slope}

In order to consider the problem of locating a line with rectangular distance and a restriction on the slope, we first give its formulation. Let $\mathcal{E}=\left\{\left(x_{1}, y_{1}\right), \ldots\right.$, $\left.\left(x_{n}, y_{n}\right)\right\}$ be the set of existing facilities. We can formulate the problem as follows:

$$
\begin{aligned}
& \min f_{l_{1}}(a, b)=\min \sum_{i=1}^{n} l_{1}\left(\left(x_{i}, y_{i}\right), l_{a, b}\right) \\
& \text { s.t. }|a| \leq s
\end{aligned}
$$

for $s \geq 0$. In the following we want to find out how a solution path for this rectangular problem looks like. In other words we are again interested in an optimal trajectory of the problem.

From now on until the end of this section let $\left(a^{*}, b^{*}\right) \in M_{v e r}^{\text {opt }}$ with $a^{*}=\min \{|a|$ : $\left.(a, b) \in M_{v e r}^{o p t}\right\}$ and $(\hat{a}, \hat{b}) \in M_{h o r}^{\text {opt }}$ with $\hat{a}=\min \left\{|a|:(a, b) \in M_{h o r}^{\text {opt }}\right\}$, respectively. In order to find out how the solution path for the rectangular problem looks like we have to distinguish the three different cases that we already mentioned in Section IV.1:

- $\left|a^{*}\right| \leq 1,|\hat{a}| \leq 1$

- $\left|a^{*}\right|>1,|\hat{a}|>1$,

- $\left|a^{*}\right| \leq 1,|\hat{a}|>1$.

Case 1: $\left|a^{*}\right| \leq 1,|\hat{a}| \leq 1$

Theorem IV.2.1. If $\left|a^{*}\right| \leq 1$ and $|\hat{a}| \leq 1$ the solution path of the rectangular problem equals exactly the solution path of the vertical problem.

Proof. We already know from Lemma IV.1.3 that in this case the optimal solutions of the unrestricted rectangular problem are identical to the optimal solutions of the unrestricted vertical problem. This means that our trajectory for the rectangular 
problem will stop in $\left(a^{*}, b^{*}\right)$. Now we have to look at the restricted problem. We know from Theorem III.2.2 that we can find trajectories for the vertical and the horizontal problem that are identical on $\left[0, a^{*}\right]$. Since the trajectory stops in $\left(a^{*}, b^{*}\right)$ this yields that the solution path of the rectangular problem and the one of the vertical problem are in fact identical. To be more precise, both optimal trajectories, the one of the vertical problem and the one of the horizontal problem, are identical to the one of the rectangular problem.

Example IV.2.1. Let $\mathbb{E}=\left\{(-1,-1),(1,1),\left(-\frac{1}{2}, \frac{1}{4}\right)\right\}$ be the set of existing facilities. It is easy to see that $\left(a^{*}, b^{*}\right)=(\hat{a}, \hat{b})=(1,0)$ and therefore $\left|a^{*}\right| \leq 1$ as well as $|\hat{a}| \leq 1$. Theorem III.2.1 yields that the solution paths of $\left(P_{v e r}\right)$ and $\left(P_{\text {hor }}\right)$ are identical on the interval $\left[0, a^{*}\right]$. Therefore the solution path of $\left(P_{l_{1}}\right)$ equals indeed the solution path of the vertical problem. In fact, in this case the rectangular solution path equals the horizontal solution path as well. All of the three different problems share the same solution path.

Case 2: $\left|a^{*}\right|>1,|\hat{a}|>1$

Theorem IV.2.2. If $\left|a^{*}\right|>1$ and $|\hat{a}|>1$ the solution path of the rectangular problem equals exactly the solution path of the horizontal problem.

Proof. We already know from Lemma IV.1.3 that in this case the optimal solutions of the unrestricted rectangular problem are identical to the optimal solutions of the unrestricted horizontal problem. Now we have to look at the restricted problem. From Theorem 11 .2.2 we know that the solution path of the vertical problem and the solution path of the horizontal problem are identical as long as $s \leq\left|a^{*}\right|$. Therefore the solution path of the rectangular problem is of course identical to both solution paths by definition of the rectangular distance.

Lemma III.2.1 yields $|\hat{a}| \geq\left|a^{*}\right|$. If $|\hat{a}|=\left|a^{*}\right|$ we are done. Let us now assume $|\hat{a}|>\left|a^{*}\right|$ and let $\left|a^{*}\right|<s \leq|\hat{a}|$. Look at $\left(P_{l_{1}}(s)\right)$ and let $(a, b) \in M_{h o r}^{\text {opt }}(s)$. Since $(a, b) \in M_{h o r}^{\text {opt }}(s)$ and $\left|a^{*}\right|>1$ the following inequalities hold: 


$$
\begin{aligned}
f_{\text {ver }}\left(a^{*}, b^{*}\right) & >\frac{1}{\left|a^{*}\right|} f_{\text {ver }}\left(a^{*}, b^{*}\right) \\
& =f_{\text {hor }}\left(a^{*}, b^{*}\right) \\
& \geq f_{\text {hor }}(a, b)
\end{aligned}
$$

Therefore for any $s$ satisfying $a^{*}<s \leq \hat{a}$ any $(a, b) \in M_{h o r}^{o p t}(s)$ is optimal for $\left(P_{l_{1}}(s)\right)$ as well. Actually this is due to the fact that the horizontal distance and the rectangular distance are identical if $a>1$.

Together this yields that the solution path of the rectangular problem and the one of the horizontal problem are in fact identical.

Example IV.2.2. Consider the given set of existing facilities $\mathcal{E}=\{(0,0),(3,5)$, $(1,6)\}$. An optimal solution for $\left(P_{\text {ver }}\right)$ is $\left(a^{*}, b^{*}\right)=\left(\frac{5}{3}, 0\right)$ while $(\hat{a}, \hat{b})=(6,0)$ is an optimal solution for $\left(P_{\text {hor }}\right)$ and $\left(P_{l_{1}}\right)$. Figure IV.2.1 shows the solution path of the rectangular problem. It is identical to the solution path of the vertical problem until $s=\frac{5}{3}$. Then it follows the solution path of the horizontal problem along the $a$-axis until it reaches the optimal solution $(6,0)$. Since the solution path of the horizontal problem is also identical to the one of the vertical problem for $s \in\left[0, a^{*}\right)$, the solution path of the rectangular problem is indeed identical to the one of the horizontal problem.

Case 3: $\left|a^{*}\right| \leq 1,|\hat{a}|>1$

This case is a bit more problematic than the two cases before. We already saw in Section IV.1 that it is not as easy to make statements about the solution path of this problem as in the two other cases.

As in the previous case we can first benefit from the fact that the solution paths of the vertical problem and the horizontal problem are identical for $s \leq\left|a^{*}\right|$. Therefore we know how the solution path of the rectangular problem looks like for these $s$. Furthermore we already know that the rectangular distance corresponds 


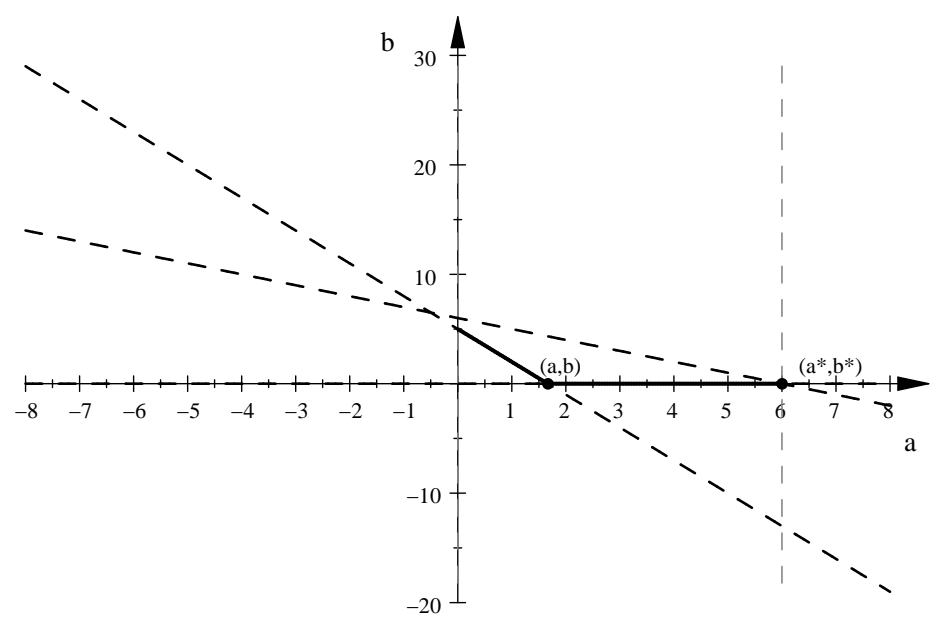

Figure IV.2.1. The solid line shows the solution path of the rectangular problem in Example IV.2.2. The point $(a, b)$ is an optimal solution to $\left(P_{v e r}\right)$ while the point $\left(a^{*}, b^{*}\right)$ is an optimal solution for $\left(P_{h o r}\right)$ as well as for $\left(P_{l_{1}}\right)$.

to the vertical distance for $a \leq 1$. But this tells us that $\left(a^{*}, b^{*}\right)$ is in fact optimal for all $\left(P_{l_{1}}(s)\right)$ with $\left|a^{*}\right| \leq s \leq 1$.

Lemma IV.2.1. The rectangular solution path equals the solution path of the vertical problem on the interval $\left[0, a^{*}\right]$ and is constantly equal to the optimal solution $\left(a^{*}, b^{*}\right)$ of $\left(P_{v e r}\right)$ on the interval $\left[a^{*}, 1\right]$.

Proof. There is nothing left to prove after our considerations above.

But what happens if $s>1$ ? We will split this case into two subcases.

a) $f_{\text {ver }}\left(a^{*}, b^{*}\right) \leq f_{\text {hor }}(\hat{a}, \hat{b})$

If $f_{v e r}\left(a^{*}, b^{*}\right) \leq f_{\text {hor }}(\hat{a}, \hat{b})$ then obviously $\left(a^{*}, b^{*}\right) \in M_{l_{1}}^{\text {opt }}$. Therefore $\left(a^{*}, b^{*}\right)$ stays optimal for $\left(P_{l_{1}}(s)\right)$ even if $s>1$. As in the first case the solution path of the rectangular problem equals the solution path of the vertical problem.

b) $f_{\text {hor }}(\hat{a}, \hat{b})<f_{v e r}\left(a^{*}, b^{*}\right)$

This second subcase is the less trivial case. We know that we still have to follow the solution path of the vertical problem until we reach $a^{*}$. Since $\left(a^{*}, b^{*}\right) \in M_{v e r}^{\text {opt }}$ and by definition of the $l_{1}$-distance it is obvious that $\left(a^{*}, b^{*}\right)$ is also optimal to any problem $\left(P_{l_{1}}(s)\right)$ with $s \in\left(\left|a^{*}\right|, 1\right]$ (see Lemma IV.2.1). This means that we get 


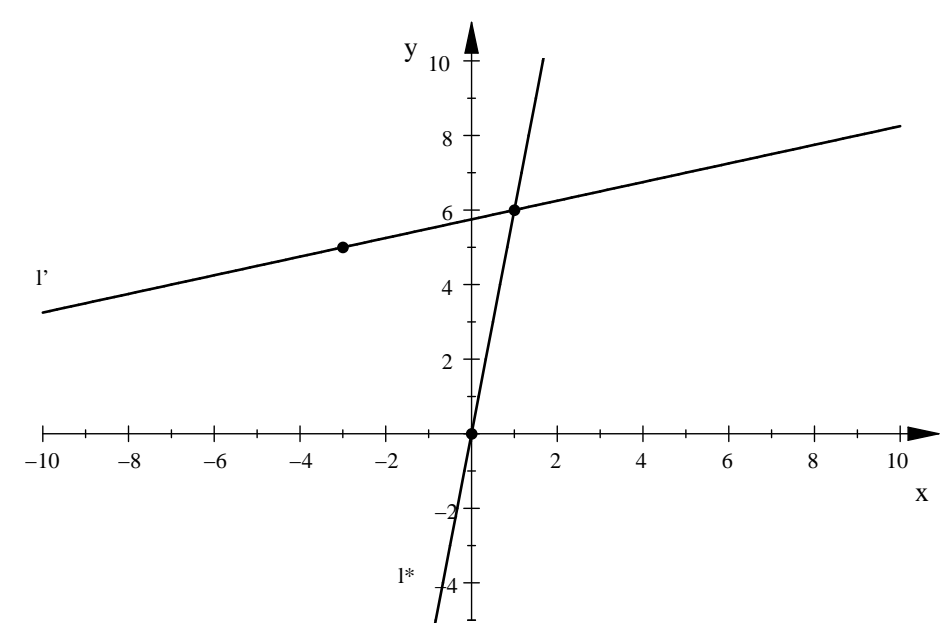

Figure IV.2.2. The existing facilities of Example IV.2.3 in the initial space. The line $l^{*}$ is an optimal line for the unrestricted problems $\left(P_{\text {hor }}\right)$ and $\left(P_{l_{1}}\right)$. The line $l^{\prime}$ is optimal for the unrestricted vertical problem $\left(P_{v e r}\right)$.

somehow stuck in the optimal solution to the vertical problem. On the other hand we know that $(\hat{a}, \hat{b})$ is optimal to $\left(P_{l_{1}}\right)$, therefore we should jump to the solution path of the horizontal problem at some point.

Example IV.2.3. Consider the set of existing facilities $\mathcal{E}=\left\{x_{1}, x_{2}, x_{3}\right\}$ with

$$
\begin{aligned}
& x_{1}=(0,0), \\
& x_{2}=(-3,5) \text { and } \\
& x_{3}=(1,6) .
\end{aligned}
$$

This is the same set of existing facilities as in Example III.1.1. The points $\left(\frac{1}{4}, \frac{23}{4}\right)$ and $(6,0)$ are optimal solutions of $\left(P_{v e r}\right)$ and $\left(P_{h o r}\right)$, respectively. Since

$$
f_{\text {hor }}(6,0)=\frac{23}{6}<\frac{23}{4}=f_{\text {ver }}\left(\frac{1}{4}, \frac{23}{4}\right)
$$

the point $(6,0)$ is an optimal solution for $\left(P_{l_{1}}\right)$ as well.

We know how the solution path of the rectangular problem looks like on the interval $[0,1]$, namely like the solution path of the vertical problem on $\left[0, \frac{1}{4}\right]$, since $\left(\frac{1}{4}, \frac{23}{4}\right)$ stays optimal for any $\left(P_{l_{1}}(s)\right)$ with $s \in\left(\frac{1}{4}, 1\right]$. What we do not know yet is how the 


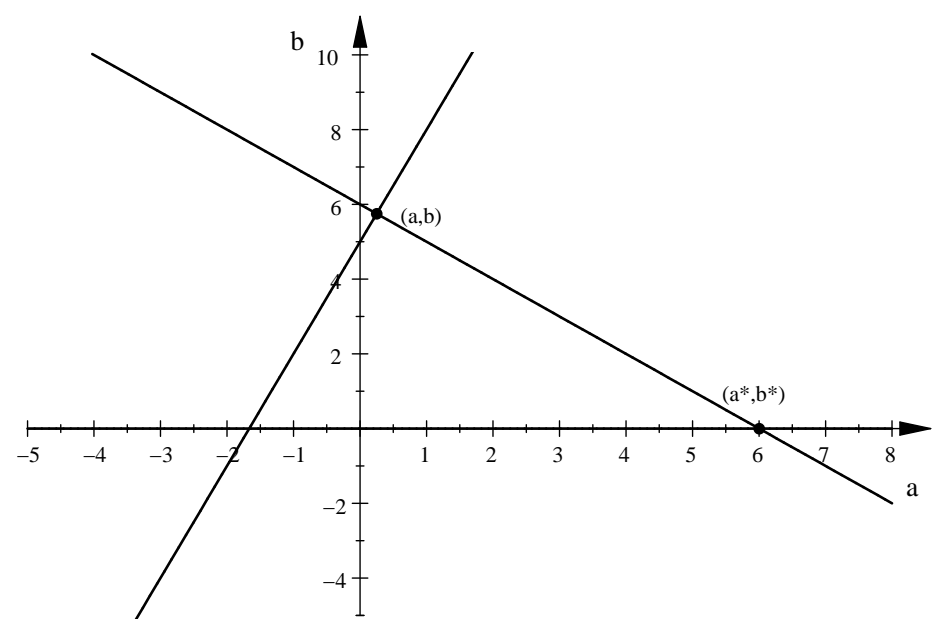

Figure IV.2.3. The dual space of Example IV.2.3 including the transformed existing facilities, an optimal solution $(a, b)$ for $\left(P_{v e r}\right)$ and an optimal solution $\left(a^{*}, b^{*}\right)$ for $\left(P_{h o r}\right)$ and $\left(P_{l_{1}}\right)$.

solution path looks like for $s \in(1,6])$, we only know that it has to end in the point $(6,0)$.

Figure IV.2.2 shows the set of existing facilities as well as optimal solutions $l^{*}$ and $l^{\prime}$ of the unrestricted rectangular problem $\left(P_{l_{1}}\right)$ and the unrestricted vertical problem $\left(P_{v e r}\right)$, respectively. The dual space including the transformed existing facilities plus the transformed optimal lines $T\left(l^{*}\right)=(\hat{a}, \hat{b})$ and $T\left(l^{\prime}\right)=\left(a^{*}, b^{*}\right)=$ is shown in Figure IV.2.3.

In order to examine the behaviour of the solution path of the rectangular problem for $s \in(1,|\hat{a}|)$ we define the following functions:

$$
\begin{aligned}
v: \mathbb{R} & \rightarrow \mathbb{R} \\
s & \mapsto \min _{b \in \mathbb{R}} f_{\text {ver }}(s, b)
\end{aligned}
$$

and

$$
\begin{aligned}
h: \mathbb{R} & \rightarrow \mathbb{R} \\
s & \mapsto \min _{b \in \mathbb{R}} f_{\text {hor }}(s, b) .
\end{aligned}
$$

These definitions make sure that $v\left(a^{*}\right)=f_{v e r}\left(a^{*}, b^{*}\right)$ and $h(\hat{a})=f_{\text {hor }}(\hat{a}, \hat{b})$. 
We define another function med $: \mathbb{R} \rightarrow \mathbb{R}$ which maps a real number $s$ to an element of the set median $\left\{\left(-x_{i} s+y_{i}, 1\right): i=1 \ldots n\right\}$ in the following way:

$$
\operatorname{med}(s)=b^{*}(s):=\min \left\{b: b \in\left(\operatorname{median}\left\{\left(-x_{i} s+y_{i}, 1\right): i=1 \ldots n\right\}\right)\right\}
$$

This means that the tuple $(s, \operatorname{med}(s))$ corresponds to the unique point lying on the median path in the dual space at $a=s$ if the number of existing facilities $n$ is odd or to the lowest point lying on the median path in the dual space at $a=s$ sometimes called the lower median if the number of existing facilities $n$ is even. In particular there always exists a line $l_{-x_{i}, y_{i}}$ passing through $(s, \operatorname{med}(s))$.

Using Theorem $[.2 .2$ and the function med defined in the above way the function $v$ can be written as

$$
\begin{aligned}
v(s) & =\min _{b} f_{v e r}(s, b) \\
& =f_{v e r}\left(s, b^{*}(s)\right) \\
& =\sum_{i=1}^{n}\left|y_{i}-x_{i} s-b^{*}(s)\right|
\end{aligned}
$$

and $h$ as

$$
\begin{aligned}
h(s) & =\min _{b} f_{h o r}(s, b) \\
& =f_{h o r}\left(s, b^{*}(s)\right) \\
& =\frac{1}{|s|} \sum_{i=1}^{n}\left|y_{i}-x_{i} s-b^{*}(s)\right| .
\end{aligned}
$$

Therefore it is of interest to know some features of $b^{*}(s)$ or in other words of the function med in order to examine the behaviour of $v$ and $h$. The following lemma provides some interesting features.

Lemma IV.2.2. The function med is piecewise linear and continuous on $\mathbb{R}$.

Proof. First we want to show that med is piecewise linear.

Let $\left(a_{1}, b_{1}\right), \ldots,\left(a_{k}, b_{k}\right)$ be the intersections of the lines $l_{-x_{i}, y_{i}}$ in the dual space and assume $a_{1}<a_{2}<\ldots<a_{k}$. Then med is linear on any interval $\left(a_{j}, a_{j+1}\right)$ for 
all $j \in\{0, \ldots, k+1\}$ if we set $a_{0}:=-\infty$ and $a_{k+1}:=\infty$ since for any $\lambda \in(0,1)$ and $s_{1}, s_{2} \in\left(a_{j}, a_{j+1}\right)$

$$
\begin{aligned}
\operatorname{med}\left(\lambda s_{1}+(1-\lambda) s_{2}\right)= & b^{*}\left(\lambda s_{1}+(1-\lambda) s_{2}\right) \\
= & \min \left\{b: b \in\left(\operatorname { m e d i a n } \left\{\left(-x_{i}\left(\lambda s_{1}+(1-\lambda) s_{2}\right)+y_{i}, 1\right):\right.\right.\right. \\
& i=1, \ldots n\})\} \\
\stackrel{(\star)}{=} & \lambda \min \left\{b: b \in\left(\operatorname{median}\left\{\left(-x_{i} s_{1}+y_{i}, 1\right): i=1 \ldots n\right\}\right)\right\} \\
& +(1-\lambda) \min \left\{b: b \in\left(\operatorname { m e d i a n } \left\{\left(-x_{i} s_{2}+y_{i}, 1\right):\right.\right.\right. \\
& i=1 \ldots n\})\} \\
= & \lambda b^{*}\left(s_{1}\right)+(1-\lambda) b^{*}\left(s_{2}\right) \\
= & \lambda \operatorname{med}\left(s_{1}\right)+(1-\lambda) \operatorname{med}\left(s_{2}\right) .
\end{aligned}
$$

The equation $(\star)$ holds since no two lines $l_{-x_{i}, y_{i}}$ and $l_{-x_{l}, y_{l}}$ intersect on the interval $\left(a_{j}, a_{j+1}\right)$.

This shows that med is a piecewise linear function and all that is left to prove is the continuity of med. Since med is piecewise linear on the intervals $\left(a_{j}, a_{j+1}\right)$ it is obvious that med is continuous on these intervals. We only have to check continuity in the $a_{j}, j=1 \ldots k$.

By definition of the $a_{j}$ and $b^{*}(s)$ it holds that there exists a line $l_{-x_{l}, y_{l}}$ such that for all $s \in\left(a_{j-1}, a_{j}\right)$ this line passes $\left(s, b^{*}(s)\right)$. In the same way there exists a line $l_{-x_{m}, y_{m}}$ such that for all $s \in\left(a_{j}, a_{j+1}\right)$ this line passes $\left(s, b^{*}(s)\right)$. Again by definition of $b^{*}$ it must hold that $l_{-x_{l}, y_{l}}$ and $l_{-x_{m}, y_{m}}$ intersect at $a_{j}$ which means exactly that

$$
\lim _{s \rightarrow a_{j}+} b^{*}(s)=\lim _{s \rightarrow a_{j}-} b^{*}(s) .
$$

Therefore med is continuous on $\mathbb{R}$.

Having that med is continuous we easily get the following proposition. 
Proposition IV.2.1. The function

$$
\begin{aligned}
v: \mathbb{R} & \rightarrow \mathbb{R} \\
s & \mapsto \sum_{i=1}^{n}\left|y_{i}-x_{i} s-b^{*}(s)\right|
\end{aligned}
$$

is continuous on $\mathbb{R}$.

Proof. Since $v$ is a composition of finitely many functions which are continuous on $\mathbb{R}$ it is continuous on $\mathbb{R}$ itself.

This proposition instantly leads to another proposition concerning the continuity of $h$.

Proposition IV.2.2. The function

$$
\begin{aligned}
h: \mathbb{R} & \rightarrow \mathbb{R} \\
s & \mapsto \frac{1}{|s|} \sum_{i=1}^{n}\left|y_{i}-x_{i} s-b^{*}(s)\right|
\end{aligned}
$$

is continuous on $\mathbb{R} \backslash\{0\}$.

Proof. $h$ is defined on $\mathbb{R} \backslash\{0\}$ and as a composition of finitely many functions which are continuous on $\mathbb{R} \backslash\{0\}$ it is continuous on that domain.

Now we can use the statement about continuity of $h$ to show that there has to exist an $a \in(1, \hat{a})$ or an $a \in(\hat{a},-1)$ in such a way that $h(a)=f_{v e r}\left(a^{*}, b^{*}\right)$.

Lemma IV.2.3. If $\left|a^{*}\right| \leq 1$ while $|\hat{a}|>1$ and $f(\hat{a}, \hat{b})<f\left(a^{*}, b^{*}\right)$ there exists an $a^{\prime} \in[1, \hat{a}]$ or an $a^{\prime} \in[\hat{a},-1]$, respectively, satisfying $h\left(a^{\prime}\right)=f_{v e r}\left(a^{*}, b^{*}\right)$.

Proof. We already know that $h(1) \geq f_{v e r}\left(a^{*}, b^{*}\right)$ and $h(-1) \geq f_{v e r}\left(a^{*}, b^{*}\right)$, respectively, and that $h(\hat{a})=f_{\text {hor }}(\hat{a}, \hat{b})<f_{\text {ver }}\left(a^{*}, b^{*}\right)$. If $h(1)=f_{\text {ver }}\left(a^{*}, b^{*}\right)$ or $h(-1)=f_{v e r}\left(a^{*}, b^{*}\right)$, respectively, then we are done. 
Let us assume $h(1)>f_{v e r}\left(a^{*}, b^{*}\right)$ and $h(-1)>f_{v e r}\left(a^{*}, b^{*}\right)$, respectively. $h$ is continuous on $\mathbb{R} \backslash\{0\}$ by Proposition IV.2.2 and therefore in particular continuous on $[1, \hat{a}]$ or $[\hat{a},-1]$, respectively. Now the intermediate value theorem makes sure that there exists an $a^{\prime} \in(1, \hat{a})$ or an $a^{\prime} \in(\hat{a},-1)$ depending on the sign of $\hat{a}$ satisfying the stated property. This completes the proof.

We will see later on that this $a^{\prime}$ is exactly the point where we move out of the minimum of the vertical problem and jump to the solution path of $\left(P_{h o r}\right)$. In order to do so we need some statements about monotonicity properties of $v$ and $h$.

Lemma IV.2.4. Assume $a>0$ for all $(a, b) \in M_{\text {ver }}^{\text {opt }}$. Then $v$ is monotonically decreasing on $\left(-\infty, a^{*}\right]$ and monotonically increasing on $\left[a^{*}, \infty\right)$.

Proof. Let $R\left(a^{\prime}\right):=\left\{a: a^{\prime} \leq a \leq \infty\right\}$ for any $a^{\prime}<0$. Since $a>0 \forall(a, b) \in M_{v e r}^{\text {opt }}$ it is $M_{v e r}^{\text {opt }} \subseteq \operatorname{int}\left(R\left(a^{\prime}\right)\right)$. Therefore the problem

$$
\begin{aligned}
& \min f_{v e r}(a, b) \\
& \text { s.t. } a \notin \operatorname{int}\left(R\left(a^{\prime}\right)\right)
\end{aligned}
$$

has an optimal solution $\left(a^{\prime}, b\left(a^{\prime}\right)\right)$ with suitable $b\left(a^{\prime}\right)$ for any $a^{\prime}<0$ according to the so called boundary theorem (Randsatz), see [Ham95]. Since any optimal solution lies on the median path (Theorem $[\mathbf{I . 2 . 2}$ ), there exists an optimal solution $\left(a^{\prime}, b^{*}\left(a^{\prime}\right)\right)$. Let $v\left(a^{\prime}\right)=f_{v e r}\left(a^{\prime}, b^{*}\left(a^{\prime}\right)\right):=z^{*}$. For $a \notin \operatorname{int}\left(R\left(a^{\prime}\right)\right)$ it must hold

$$
v(a)=f_{v e r}\left(a, b^{*}(a)\right) \geq z^{*}
$$

Therefore it holds for all $a<a^{\prime}$ that $v(a) \geq v\left(a^{\prime}\right)$ which means that $v$ is monotonically decreasing on $(-\infty, 0)$.

From Section $\left[.2\right.$ it is already known that $v$ is monotonically decreasing on $\left[0, a^{*}\right]$. To complete the proof we have to show that $v$ is monotonically increasing on $\left(a^{*}, \infty\right)$.

Let $\left(a_{\text {max }}^{*}, b_{\text {max }}^{*}\right) \in M_{v e r}^{\text {opt }}$ such that $a_{\text {max }}^{*}=\max \left\{a:(a, b) \in M_{v e r}^{\text {opt }}\right\}$.

If $a^{\prime} \in\left(a^{*}, a_{\text {max }}^{*}\right]$ then $v\left(a^{\prime}\right)=v\left(a^{*}\right)=v\left(a_{\text {max }}^{*}\right)$ since $f_{v e r}$ is convex in $a$ and $\left(a^{*}, b^{*}\right),\left(a_{\text {max }}^{*} b_{\text {max }}^{*}\right) \in M_{\text {ver }}^{\text {opt }}$. 


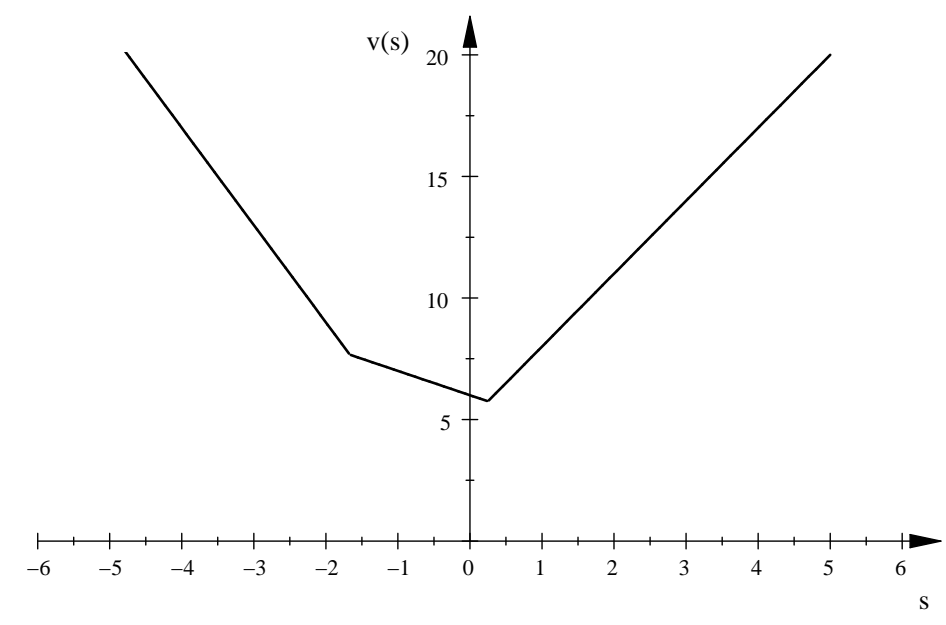

Figure IV.2.4. The function $v$ for Example IV.2.3. One can easily see that $v$ is continuous and quasiconvex on $\mathbb{R}$. It reaches its minimum in $\left(a^{*}, v\left(a^{*}\right)\right)=\left(\frac{1}{4}, \frac{23}{4}\right)$ where $v\left(a^{*}\right)=f_{\text {ver }}\left(a^{*}, b^{*}\right)$. On the interval $\left(-\infty, \frac{1}{4}\right) v$ is monotonically decreasing while it is monotonically increasing on $\left(\frac{1}{4}, \infty\right)$.

If $a^{\prime}>a_{\max }^{*}$ then let $R\left(a^{\prime}\right):=\left\{a:-\infty \leq a \leq a^{\prime}\right\}$. Again we use the boundary theorem to complete the proof. It is true that $M_{v e r}^{\text {opt }} \subseteq \operatorname{int}\left(R\left(a^{\prime}\right)\right)$. Therefore the problem

$$
\begin{aligned}
& \min f_{v e r}(a, b) \\
& \text { s.t. } a \notin \operatorname{int}\left(R\left(a^{\prime}\right)\right)
\end{aligned}
$$

has an optimal solution $\left(a^{\prime}, b^{*}\left(a^{\prime}\right)\right)$. But since $a \notin \operatorname{int}\left(R\left(a^{\prime}\right)\right)$ for all $a \geq a^{\prime}$ it holds that

$$
v(a)=f_{v e r}\left(a, b^{*}(a)\right) \geq f_{v e r}\left(a^{\prime}, b^{*}\left(a^{\prime}\right)\right)=v\left(a^{\prime}\right) .
$$

Therefore $v$ is monotonically increasing on $\left(a^{*}, \infty\right)$ which completes the proof.

Remark IV.2.1. In the case $a<0 \forall(a, b) \in M_{\text {ver }}^{\text {opt }}$ we can prove analogously that $v$ is monotonically decreasing on $\left(-\infty, a_{\max }^{*}\right)$ and monotonically increasing on $\left(a_{\text {max }}^{*}, \infty\right)$ using a slightly different definition of $a_{\max }^{*}: a_{\max }^{*}=\max \{|a|:(a, b) \in$ $\left.M_{\text {ver }}^{o p t}\right\}$.

The previous lemma has the nice side effect that we easily get the following proposition. 
Proposition IV.2.3. $v$ is quasiconvex on $\mathbb{R}$.

Proof. Any function decreasing up to a point and increasing from that point on is quasiconvex (see e.g. [Jun08]).

We still need some information about monotonicity of $h$. The following lemma provides the information needed.

Lemma IV.2.5. If $a>0 \forall(a, b) \in M_{\text {hor }}^{\text {opt }}$ then $h$ is monotonically decreasing on $(0, \hat{a}]$ and monotonically increasing on $[\hat{a}, \infty)$ as well as monotonically increasing on $(-\infty, 0)$.

If $a<0 \forall(a, b) \in M_{\text {hor }}^{\text {opt }}$ then $h$ is monotonically decreasing on $(-\infty, \hat{a}]$ and monotonically increasing on $[\hat{a}, 0)$ as well as monotonically decreasing on $(0, \infty)$.

Proof. Assume $a>0$ for all $(a, b) \in M_{h o r}^{o p t}$. This means that $\hat{a}>0$ in particular.

We want to use the fact that we know about the monotonicity properties of $v$ due to Lemma IV.2.4 in order to show the monotonicity properties of $h$. We know that

$$
\begin{aligned}
h(a) & =\min _{b \in \mathbb{R}} f_{\text {hor }}(a, b) \\
& =\min _{\beta \in \mathbb{R}} f_{\text {ver }}\left(-\frac{1}{a}, \beta\right) \\
& =\min _{\beta \in \mathbb{R}} f_{\text {ver }}(\alpha, \beta)
\end{aligned}
$$

with $\alpha=-\frac{1}{a}$ because the horizontal distance in our initial space is equal to the vertical distance in the rotated space. Let

$$
\begin{aligned}
\tilde{v}: \mathbb{R} & \rightarrow \mathbb{R} \\
\tilde{s} & \mapsto \min _{\beta \in \mathbb{R}} f_{\text {ver }}(\tilde{s}, \beta)
\end{aligned}
$$

with $\tilde{s}=\frac{1}{s}$ be the function in the rotated space that corresponds to $v$ in the initial space. Then it has to hold that

$$
\begin{aligned}
h(a) & =\min _{\beta \in \mathbb{R}} f_{v e r}(\alpha, \beta) \\
& =\tilde{v}(\alpha)
\end{aligned}
$$


due to Theorem III.1.4.

We know that $\hat{a} \in M_{\text {hor }}^{\text {opt }}$ and $\hat{a}>0$ by assumption. This tells us that $\hat{\alpha}=$ $-\frac{1}{\hat{a}}<0$ is an optimal solution to $\left(\tilde{P}_{v e r}\right)$ and by definition of $\hat{a}$ it holds that $\hat{\alpha}=\max \left\{|\alpha|: \quad(\alpha, \beta) \in \tilde{M}_{v e r}^{\text {opt }}\right\}$. Lemma IV.2.4 or rather Remark IV.2.1 tell us that $\tilde{v}$ is monotonically increasing on $[\hat{\alpha}, \infty)$ and monotonically decreasing on $(-\infty, \hat{\alpha}]$.

First we want to show that $h$ is monotonically decreasing on $(0, \hat{a}]$.

Let $\left(a_{1}, b^{*}\left(a_{1}\right)\right) \in M_{h o r}^{\text {opt }}\left(a_{1}\right)$ and $\left(a_{2}, b^{*}\left(a_{2}\right)\right) \in M_{h o r}^{\text {opt }}\left(a_{2}\right)$ such that

$$
0<a_{1}<a_{2} \leq \hat{a}
$$

This means that

$$
h\left(a_{1}\right)=\tilde{v}\left(\alpha_{1}\right) \text { and } h\left(a_{2}\right)=\tilde{v}\left(\alpha_{2}\right)
$$

with $\alpha_{1}=-\frac{1}{a_{1}}$ and $\alpha_{2}=-\frac{1}{a_{2}}$, respectively. Note that in the rotated space it holds that

$$
\alpha_{1}<\alpha_{2} \leq \hat{\alpha}<0
$$

Since $\tilde{v}$ is monotonically decreasing on $(-\infty, \hat{\alpha}]$ it must hold that

$$
\tilde{v}\left(\alpha_{1}\right) \geq \tilde{v}\left(\alpha_{2}\right)
$$

which directly gives us

$$
h\left(a_{1}\right) \geq h\left(a_{2}\right)
$$

and therefore $h$ is monotonically decreasing on $(0, \hat{a}]$.

Next we want to show that $h$ is monotonically increasing on $[\hat{a}, \infty)$. Let therefore $\left(a_{1}, b^{*}\left(a_{1}\right)\right),\left(a_{2}, b^{*}\left(a_{2}\right)\right) \in \mathbb{R}^{2}$ such that

$$
0<\hat{a} \leq a_{1}<a_{2}
$$

It is

$$
h\left(a_{1}\right)=\tilde{v}\left(\alpha_{1}\right) \text { as well as } h\left(a_{2}\right)=\tilde{v}\left(\alpha_{2}\right) .
$$


In the rotated space it holds that

$$
\hat{\alpha}<\alpha_{1}<\alpha_{2}<0
$$

Since $\tilde{v}$ is monotonically increasing on $[\hat{\alpha}, \infty)$ it holds that

$$
\tilde{v}\left(\alpha_{1}\right) \leq \tilde{v}\left(\alpha_{2}\right)
$$

and therefore

$$
h\left(a_{1}\right) \leq h\left(a_{2}\right)
$$

as well. It follows that $h$ is monotonically increasing on $[\hat{a}, \infty)$.

Finally we want to show that $h$ is monotonically increasing on $(-\infty, 0)$. Let now $\left(a_{1}, b^{*}\left(a_{1}\right)\right),\left(a_{2}, b^{*}\left(a_{2}\right)\right) \in \mathbb{R}^{2}$ such that

$$
a_{1}<a_{2}<0
$$

Again it is

$$
h\left(a_{1}\right)=\tilde{v}\left(\alpha_{1}\right) \text { as well as } h\left(a_{2}\right)=\tilde{v}\left(\alpha_{2}\right)
$$

This gives us

$$
0<\alpha_{1}<\alpha_{2}
$$

in the rotated space. We know that $\tilde{v}$ is monotonically increasing on $(0, \infty)$ and therefore

$$
\tilde{v}\left(\alpha_{1}\right) \leq \tilde{v}\left(\alpha_{2}\right)
$$

In the end we get that

$$
h\left(a_{1}\right) \leq h\left(a_{2}\right),
$$

in other words we get that $h$ is monotonically increasing on $(-\infty, 0)$.

This is all we had to show. If $a<0 \forall(a, b) \in M_{h o r}^{\text {opt }}$ our statements can be proven analogously.

Corollary IV.2.1. Let $\left|a^{*}\right| \leq 1$ while $|\hat{a}|>1$ and $f(\hat{a}, \hat{b})<f\left(a^{*}, b^{*}\right)$ and let $a^{\prime}$ be the real number satisfying $h\left(a^{\prime}\right)=f_{\text {ver }}\left(a^{*}, b^{*}\right)$ which exists due to LemmaIV.2.3. Then the solution path of the rectangular problem is equal to the solution path of 


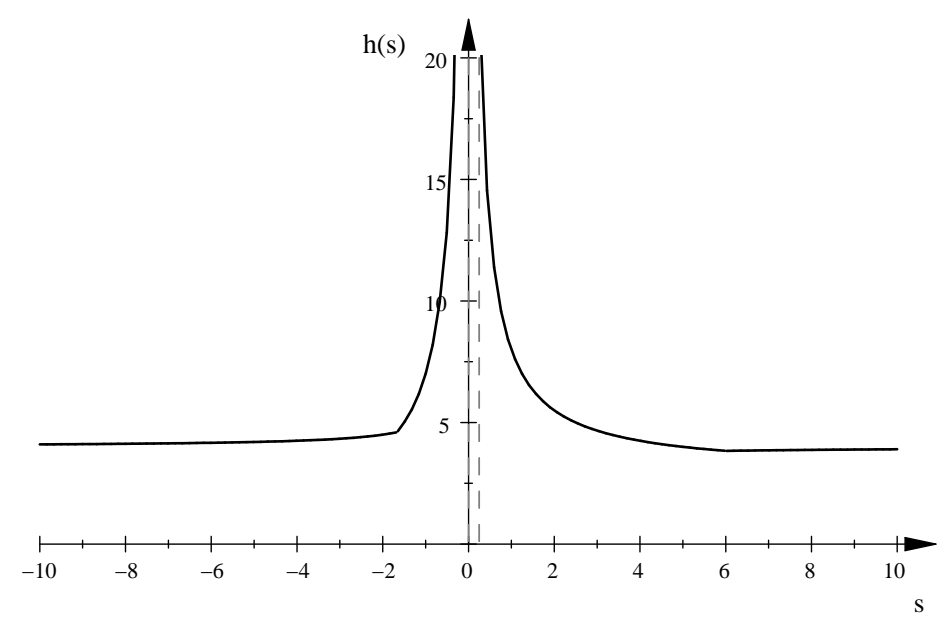

Figure IV.2.5. The function $h$ for Example IV.2.3, $h$ is continuous on $\mathbb{R} \backslash\{0\}$ and reaches its minimum in $\left(\hat{a}, g(\hat{a})=\left(6, \frac{23}{6}\right)\right.$ where $h(\hat{a})=f_{\text {hor }}(\hat{a}, \hat{b})$. On the intervals $(-\infty, 0)$ and $(6, \infty) h$ is monotonically increasing while it is monotonically decreasing on $(0,6)$.

the vertical problem on the interval $\left[0,\left|a^{\prime}\right|\right]$ and to the one of the horizontal problem on $\left[\left|a^{\prime}\right|,|\hat{a}|\right]$.

Proof. Right at the beginning of this case it is stated that the solution paths of $\left(P_{l_{1}}\right)$ and $\left(P_{v e r}\right)$ are the same on $\left[0, a^{*}\right]$ or $\left[a^{*}, 0\right]$, respectively, depending on whether $a>0$ for all $(a, b) \in M_{v e r}^{\text {opt }}$ or $a<0$ for all $(a, b) \in M_{v e r}^{\text {opt }}$, and that $\left(a^{*}, b^{*}\right)$ stays optimal for $\left(P_{l_{1}}(s)\right)$ as long as $s \leq 1$. Moreover, since $h$ is monotonically decreasing on $(0,|\hat{a}|]$ and therefore on $[1,|\hat{a}|]$ in particular and monotonically increasing on $[-|\hat{a}|, 0)$ and therefore on $[-|\hat{a}|,-1]$ in particular (Lemma IV.2.5),$\left(a^{*}, b^{*}\right)$ even stays optimal for $\left(P_{l_{1}}(s)\right)$ until $s$ reaches $\left|a^{\prime}\right|$, where $a^{\prime}$ is the real number described in Lemma IV.2.3 satisfying $h\left(a^{\prime}\right)=f_{\text {ver }}\left(a^{*}, b^{*}\right)$. Again by monotonicity of $h$ we get that $h(s) \leq f_{\text {ver }}\left(a^{*}, b^{*}\right)$ for any $s$ with $s \in\left(\left|a^{\prime}\right|,|\hat{a}|\right]$. This means exactly that we switch from the solution path of the vertical problem to the one of the horizontal problem as soon as we reach $\left|a^{\prime}\right|$.

At this point we are able to put all the achievements up to now together to get a solution path for $\left(P_{l_{1}}\right)$ : We follow the solution path of the vertical problem until we reach $s=\left|a^{*}\right|$ (Lemma IV.2.1). $\left(a^{*}, b^{*}\right)$ stays optimal for $P_{l_{1}}(s)$ until $s$ reaches $\left|a^{\prime}\right|$ (Corollary IV.2.1). At $s=\left|a^{\prime}\right|$ we get $\left(a^{\prime}, b^{*}\left(a^{\prime}\right)\right)$ as an optimal 
solution to $\left(P_{l_{1}}\left(\left|a^{\prime}\right|\right)\right)$ satisfying $f_{l_{1}}\left(a^{*}, b^{*}\right)=f_{l_{1}}\left(a^{\prime}, b^{*}\left(a^{\prime}\right)\right)$. From now on we follow the solution path of the horizontal problem improving the value of the objective function until we reach the optimal solution $(\hat{a}, \hat{b})$ (again Corollary IV.2.1).

Remark IV.2.2. It is important to note that most of the results concerning properties of the functions $v, h$ and med are general results even if stated during the examination of the special Case 3. Only LemmaIV.2.3 and Corollary IV.2.1 make use of the additional assumptions while all other results concerning $v, h$ and med are of general nature.

Up to now we only stated the existence of such an $a^{\prime}$. There is no doubt that it would be even better to know how to find it. Therefore we will finish this section demonstrating how to construct $a^{\prime}$ as a convex combination of two known points. We search for an $a^{\prime}$ that satisfies $f_{\text {hor }}\left(a^{\prime}, b^{*}\left(a^{\prime}\right)\right)=f_{\text {ver }}\left(a^{*}, b^{*}\right)$ while at the same time $\left(a^{\prime}, b^{*}\left(a^{\prime}\right)\right)$ is an optimal solution for the problem

$$
\begin{aligned}
& \min f_{\text {hor }}(a, b) \\
& \text { s.t. }|a| \leq\left|a^{\prime}\right| .
\end{aligned}
$$

What we already know is that $\left(a^{\prime}, b^{*}\left(a^{\prime}\right)\right)$ has to correspond to a pseudo-halving line and therefore has to lie on the median path in the dual space (see Lemma III.1.1). Furthermore $\left(a^{*}, b^{*}\right)$ and $(\hat{a}, \hat{b})$ are in each case intersections of at least two lines corresponding to existing facilities in the dual space (see Section I.1) and the following inequalities hold:

$$
\begin{gathered}
f_{\text {ver }}\left(a^{*}, b^{*}\right)<f_{\text {hor }}\left(a^{*}, b^{*}\right), \\
f_{\text {hor }}(\hat{a}, \hat{b})<f_{\text {ver }}\left(a^{*}, b^{*}\right) .
\end{gathered}
$$

Let $A:=\left\{(a, b):\left|a^{*}\right| \leq|a| \leq|\hat{a}|,(a, b)\right.$ being an intersection of at least two lines in the dual space lying on the (lower) median path\}. Using our definition of 
$b^{*}(a)$ we can rewrite $A$ as

$$
A:=\left\{\left(a, b^{*}(a)\right):\left|a^{*}\right| \leq|a| \leq|\hat{a}|,\left(a, b^{*}(a)\right)\right. \text { being an intersection of at least }
$$
two lines in the dual space\}.

Then $A \neq \emptyset$ because $\left(a^{*}, b^{*}\right),(\hat{a}, \hat{b}) \in A$. Since $A$ has finitely many elements we write $A$ as $A=\left\{\left(a_{i}, b^{*}\left(a_{i}\right)\right): i=1, \ldots, k, k \in \mathbb{N}\right\}$ with $a_{1}=a^{*}$ and $a_{k}=\hat{a}$.

Assume without loss of generality that the elements of $A$ are sorted in such a way that

$$
\left|a^{*}\right|=\left|a_{1}\right|<\left|a_{2}\right|<\ldots<\left|a_{k}\right|=|\hat{a}| .
$$

Now we check for every $\left(a_{i}, b^{*}\left(a_{i}\right)\right) \in A$ whether $f_{\text {hor }}\left(a_{i}, b^{*}\left(a_{i}\right)\right)>f_{\text {ver }}\left(a^{*}, b^{*}\right)$ or $f_{\text {hor }}\left(a_{i}, b^{*}\left(a_{i}\right)\right)<f_{\text {ver }}\left(a^{*}, b^{*}\right)$. If there is a point $\left(a_{i}, b^{*}\left(a_{i}\right)\right)$ in $A$ satisfying $f_{\text {hor }}\left(a_{i}, b_{i}\right)=f_{\text {ver }}\left(a^{*}, b^{*}\right)$ we are done.

We choose $\left(a_{j}, b^{*}\left(a_{j}\right)\right)$ in such a way that

$$
\left|a_{j}\right|=\min \left\{\left|a_{i}\right|:\left(a_{i}, b^{*}\left(a_{i}\right)\right) \in A, f_{\text {hor }}\left(a_{i}, b_{i}\right)<f_{\text {ver }}\left(a^{*}, b^{*}\right)\right\}
$$

Such an $\left(a_{j}, b^{*}\left(a_{j}\right)\right)$ always exists since $(\hat{a}, \hat{b}) \in A$ and $f_{\text {hor }}(\hat{a}, \hat{b})<f_{\text {ver }}\left(a^{*}, b^{*}\right)$.

By the way we have chosen $\left(a_{j}, b^{*}\left(a_{j}\right)\right)$ we know that

$$
\begin{aligned}
& f_{\text {hor }}\left(a_{j}, b^{*}\left(a_{j}\right)\right)<f_{\text {ver }}\left(a^{*}, b^{*}\right) \text { and } \\
& f_{\text {hor }}\left(a_{i}, b^{*}\left(a_{i}\right)\right)>f_{\text {ver }}\left(a^{*}, b^{*}\right) \forall i<j .
\end{aligned}
$$

Now we choose the "neighbour" $\left(a_{N}, b^{*}\left(a_{N}\right)\right)$ of $\left(a_{j}, b^{*}\left(a_{j}\right)\right)$ that satisfies

$$
f_{\text {hor }}\left(a_{N}, b^{*}\left(a_{N}\right)\right)>f_{v e r}\left(a^{*}, b^{*}\right)
$$

by

$$
a_{N}=\max \left\{a_{i}:\left|a_{i}\right|<\left|a_{j}\right| \text { and } \operatorname{sgn}\left(a_{N}\right)=\operatorname{sgn}\left(a_{j}\right)\right\}
$$

if such an $a_{N}$ exists or as $a_{N}=a^{*}$ otherwise. The point $\left(a_{N}, b^{*}\left(a_{N}\right)\right)$ satisfies $f_{\text {hor }}\left(a_{N}, b^{*}\left(a_{N}\right)\right)>f_{v e r}\left(a^{*}, b^{*}\right)$ since by choice of $a_{N}$ it must hold that $N<j$. There exists no intersection $(a, b)$ of two or more lines lying on the (lower) median 
path satisfying $\left|a_{N}\right|<|a|<\left|a_{j}\right|$ and $\operatorname{sgn}(a)=\operatorname{sgn}\left(a_{j}\right)$, therefore there exists a line $l_{-x_{l}, y_{l}} \in\left\{l_{-x_{i}, y_{i}}: i=1, \ldots, n\right\}$ passing through $\left(a_{N}, b^{*}\left(a_{N}\right)\right)$ as well as $\left(a_{j}, b_{j}\right)$ in such a way that any point $(a, b)$ satisfying $\left|a_{N}\right|<|a|<\left|a_{j}\right|$ and $\operatorname{sgn}(a)=\operatorname{sgn}\left(a_{j}\right)$ which lies on the (lower) median path can be written as $\left(a,-x_{l} a+y_{l}\right)$.

This means exactly that there exists a line $l_{-x_{l}, y_{l}}$ representing the section of the (lower) median path between $a_{N}$ and $a_{j}$.

Furthermore any $a$ satisfying $\left|a_{N}\right|<|a|<\left|a_{j}\right|$ and $\operatorname{sgn}(a)=\operatorname{sgn}\left(a_{j}\right)$ can be written as a convex combination of $a_{N}$ and $a_{j}$, that is to say as $\lambda a_{N}+(1-\lambda) a_{j}$ with a $\lambda \in(0,1)$.

Putting this together we can write any point $(a, b)$ satisfying $\left|a_{N}\right|<|a|<\left|a_{j}\right|$ and $\operatorname{sgn}(a)=\operatorname{sgn}\left(a_{j}\right)$ which lies on the (lower) median path as

$$
\left(\lambda a_{N}+(1-\lambda) a_{j},-\left(\lambda a_{N}+(1-\lambda) a_{j}\right) x_{l}+y_{l}\right) \text { with } \lambda \in(0,1) .
$$

We are now searching for a $\bar{\lambda} \in(0,1)$ which yields

$$
f_{\text {hor }}\left(\bar{\lambda} a_{N}+(1-\bar{\lambda}) a_{j},-\left(\bar{\lambda} a_{N}+(1-\bar{\lambda}) a_{j}\right) x_{l}+y_{l}\right)=f_{\text {ver }}\left(a^{*}, b^{*}\right) .
$$

Such a $\bar{\lambda}$ has to exist since $f_{\text {hor }}\left(a_{j}, b^{*}\left(a_{j}\right)\right)<f_{\text {ver }}\left(a^{*}, b^{*}\right)$ and $f_{\text {hor }}\left(a_{N}, b^{*}\left(a_{N}\right)\right)>$ $f_{\text {ver }}\left(a^{*}, b^{*}\right)$ while $f_{\text {hor }}$ is continuous on $\left(a_{N}, a_{j}\right)$ if $\operatorname{sgn}\left(a_{N}\right)=\operatorname{sgn}\left(a_{j}\right)=1$ or on $\left(a_{j}, a_{N}\right)$ if $\operatorname{sgn}\left(a_{N}\right)=\operatorname{sgn}\left(a_{j}\right)=-1$, respectively.

We use the sets

$$
L:=\left\{i:-x_{i}\left(\lambda a_{N}+(1-\lambda) a_{j}\right)+y_{i}<-x_{l}\left(\lambda a_{N}+(1-\lambda) a_{j}\right)+y_{l} \forall \lambda \in(0,1)\right\}
$$

and

$$
R:=\left\{i:-x_{i}\left(\lambda a_{N}+(1-\lambda) a_{j}\right)+y_{i}>-x_{l}\left(\lambda a_{N}+(1-\lambda) a_{j}\right)+y_{l} \forall \lambda \in(0,1)\right\}
$$


to derive an alternative formulation of $f_{v e r}\left(\lambda a_{N}+(1-\lambda) a_{j},-\left(\lambda a_{N}+(1-\lambda) a_{j}\right) x_{l}+\right.$ $\left.y_{l}\right)$ :

$$
\begin{gathered}
f_{v e r}\left(\lambda a_{N}+(1-\lambda) a_{j},-\left(\lambda a_{N}+(1-\lambda) a_{j}\right) x_{l}+y_{l}\right) \\
=\sum_{i \in R} \lambda\left(a_{j}-a_{N}\right) \cdot x_{i}-a_{j} x_{i}+y_{i} \\
-|R| \cdot\left(\lambda \cdot\left(a_{j}-a_{N}\right) \cdot x_{l}-a_{j} x_{l}+y_{l}\right) \\
+|L| \cdot\left(\lambda \cdot\left(a_{j}-a_{N}\right) \cdot x_{l}-a_{j} x_{l}+y_{l}\right) \\
-\sum_{i \in L} \lambda\left(a_{j}-a_{N}\right) \cdot x_{i}-a_{j} x_{i}+y_{i}
\end{gathered}
$$

All we have to do now to get a formula for $\bar{\lambda}$ is to substitute the formula for $f_{v e r}$ above into $(\star)$ and solve the equation for $\bar{\lambda}$.

For the sake of a simplified notation we put $z^{*}:=f_{\text {ver }}\left(a^{*}, b^{*}\right)$. We can get rid of the absolute value in $(\star)$ by putting

$$
\begin{aligned}
\left|\bar{\lambda} a_{N}+(1-\bar{\lambda}) a_{j}\right| & =\operatorname{sgn}\left(a_{j}\right) \bar{\lambda} a_{N}+\operatorname{sgn}\left(a_{j}\right)(1-\bar{\lambda}) a_{j} \\
& =\operatorname{sgn}\left(a_{j}\right)\left(\bar{\lambda} a_{N}+(1-\bar{\lambda}) a_{j}\right)
\end{aligned}
$$

If $\operatorname{sgn}\left(a_{N}\right)=\operatorname{sgn}\left(a_{j}\right)$ this is easy to see. But this is also also true if $\operatorname{sgn}\left(a_{N}\right) \neq$ $\operatorname{sgn}\left(a_{j}\right)$.

Assume for instance $\operatorname{sgn}\left(a_{j}\right)=-1$ and $\operatorname{sgn}\left(a_{N}\right)=1$. By choice of $a_{N}$ we know that in this case $a_{N}=a^{*}$. Then $\left|\bar{\lambda} a_{N}+(1-\bar{\lambda}) a_{j}\right|>1$ since otherwise

$$
f_{v e r}\left(\bar{\lambda} a_{N}+(1-\bar{\lambda}) a_{j},-\left(\bar{\lambda} a_{N}+(1-\bar{\lambda}) a_{j}\right) x_{l}+y_{l}\right)<f_{v e r}\left(a^{*}, b^{*}\right)
$$

due to 


$$
\begin{aligned}
& f_{\text {hor }}\left(\bar{\lambda} a_{N}+(1-\bar{\lambda}) a_{j},-\left(\bar{\lambda} a_{N}+(1-\bar{\lambda}) a_{j}\right) x_{l}+y_{l}\right) \\
& =\frac{1}{\left|\bar{\lambda} a_{N}+(1-\bar{\lambda}) a_{j}\right|} f_{v e r}\left(\bar{\lambda} a_{N}+(1-\bar{\lambda}) a_{j},-\left(\bar{\lambda} a_{N}+(1-\bar{\lambda}) a_{j}\right) x_{l}+y_{l}\right) \\
& =f_{v e r}\left(a^{*}, b^{*}\right)
\end{aligned}
$$

and $\frac{1}{\left|\lambda a_{N}+(1-\lambda) a_{j}\right|}>1$. The last equation is due to $(\star)$. This is a contradiction to optimality of $\left(a^{*}, b^{*}\right)$. Since we assumed that $\left|a^{*}\right| \leq 1$ and we know that $a_{N}=a^{*}$ it has to hold that $\operatorname{sgn}\left(\bar{\lambda} a_{N}+(1-\bar{\lambda}) a_{j}\right)=-1$ and $\left|\bar{\lambda} a_{N}+(1-\bar{\lambda}) a_{j}\right|>1$.

Therefore it is actually true that

$$
\begin{aligned}
\left|\bar{\lambda} a_{N}+(1-\bar{\lambda}) a_{j}\right| & =\operatorname{sgn}\left(\bar{\lambda} a_{N}+(1-\bar{\lambda}) \cdot\left(\bar{\lambda} a_{N}+(1-\bar{\lambda}) a_{j}\right)\right. \\
& =(-1) \cdot\left(\bar{\lambda} a_{N}+(1-\bar{\lambda}) a_{j}\right) \\
& =-\bar{\lambda} a_{N}-(1-\bar{\lambda}) a_{j} \\
& =\operatorname{sgn}\left(a_{j}\right) \bar{\lambda} a_{N}+\operatorname{sgn}\left(a_{j}\right)(1-\bar{\lambda}) a_{j} .
\end{aligned}
$$

In the case $\operatorname{sgn}\left(a_{j}\right)=-1$ and $\operatorname{sgn}\left(a_{N}\right)=1$ the argumentation is analogous.

In the end we obtain the following formula to calculate $\bar{\lambda}$ :

$$
\bar{\lambda}=\frac{z_{1}}{z_{2}}
$$

with

$z_{1}:=z^{*} \operatorname{sgn}\left(a_{j}\right) a_{j}+\sum_{i \in L}\left(-a_{j} x_{i}+y_{i}\right)-\sum_{i \in R}\left(-a_{j} x_{i}+y_{i}\right)-(|L|-|R|) \cdot\left(-a_{j} x_{l}+y_{l}\right)$

and

$$
\begin{aligned}
z_{2}:= & -z^{*} \cdot\left(\operatorname{sgn}\left(a_{j}\right) a_{N}-\operatorname{sgn}\left(a_{j}\right) a_{j}\right)+\sum_{i \in R}\left(\left(a_{j}-a_{N}\right) \cdot x_{i}\right)-\sum_{i \in L}\left(\left(a_{j}-a_{N}\right) \cdot x_{i}\right) \\
& +(|L|-|R|) \cdot\left(\left(-a_{N}+a_{j}\right) \cdot x_{l}\right) .
\end{aligned}
$$




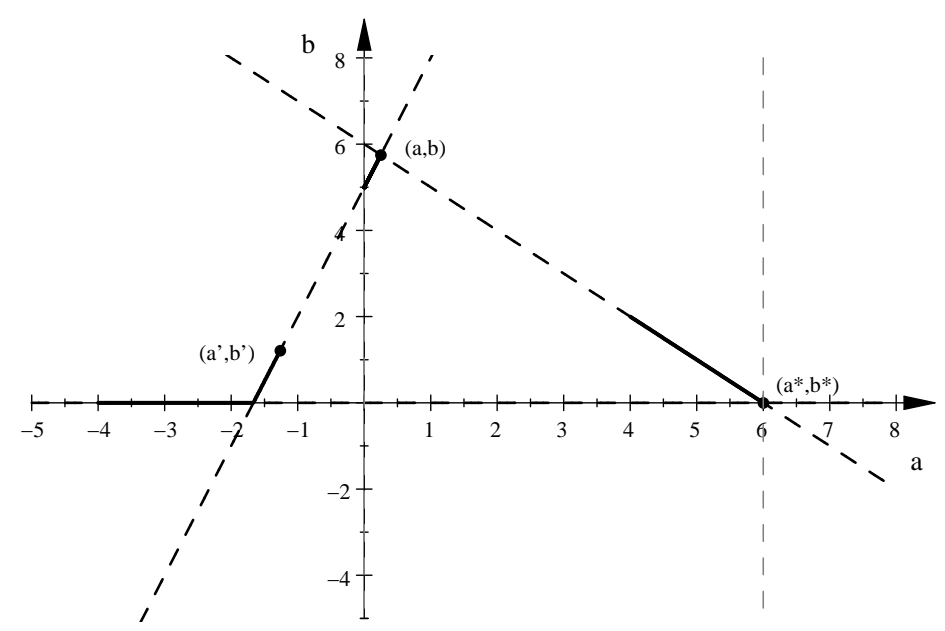

Figure IV.2.6. Solution path in the dual space for the rectangular problem in Example IV.2.3. The point $(a, b)$ is an optimal solution to $\left(P_{v e r}\right)$, the point $\left(a^{\prime}, b^{\prime}\right)$ is a point as described in Lemma IV.2.3 and $\left(a^{*}, b^{*}\right)$ is an optimal solution to $\left(P_{h o r}\right)$ as well as to $\left(P_{l_{1}}\right)$.

Example IV.2.4. We want to calculate $a^{\prime}$ for our Example IV.2.3. A look at FigureIV.2.3 might be helpful.

First we have to determine the set $A$. In our case, $A=\left\{\left(\frac{1}{4}, \frac{23}{4}\right),\left(-\frac{5}{3}, 0\right),(6,0)\right\}$. Note that $\left(\frac{1}{4}, \frac{23}{4}\right)=\left(a^{*}, b^{*}\right)$ and $(6,0)=(\hat{a}, \hat{b})$. Obviously

$$
f_{\text {hor }}\left(a^{*}, b^{*}\right)>f_{\text {ver }}\left(a^{*}, b^{*}\right)
$$

and

$$
f_{\text {hor }}(\hat{a}, \hat{b})<f_{v e r}\left(a^{*}, b^{*}\right) .
$$

Furthermore it is

$$
f_{\text {hor }}\left(-\frac{5}{3}, 0\right)=\frac{23}{5}<\frac{23}{4}=f_{v e r}\left(a^{*}, b^{*}\right) .
$$

Therefore we choose $\left(-\frac{5}{3}, 0\right)$ as $\left(a_{j}, b^{*}\left(a_{j}\right)\right)$. Since there is no other $a_{i} \in A$ with $\operatorname{sgn}\left(a_{i}\right)=-1$ we choose $\left(\frac{1}{4}, \frac{23}{4}\right)$ as $\left(a_{N}, b^{*}\left(a_{N}\right)\right)$. The line $l_{-x_{l}, y_{l}}$ passing $\left(a_{N}, b^{*}\left(a_{N}\right)\right)$ and $\left(a_{j}, b^{*}\left(a_{j}\right)\right)$ is $l_{3,5}$, therefore $R=\{3\}$ and $L=\{1\}$.

This is all we have to know to calculate $\bar{\lambda}$ using the formula above: 


$$
\begin{aligned}
\bar{\lambda} & =\frac{\frac{23}{4} \cdot \frac{5}{3}+\frac{5}{3} \cdot 0+0-\left(\frac{5}{3} \cdot 1+6\right)-(1-1) \cdot\left(\frac{5}{3} \cdot(-3)+5\right)}{-\frac{23}{4} \cdot\left(-\frac{1}{4}-\frac{5}{3}\right)+\left(-\frac{5}{3}-\frac{1}{4}\right) \cdot 1-\left(\left(-\frac{5}{3}-\frac{1}{4}\right) \cdot 0\right)+(1-1)\left(-\frac{1}{4}-\frac{5}{3}\right) \cdot(-3)} \\
& =\frac{4}{19}
\end{aligned}
$$

Therefore we get

$$
a^{\prime}=\bar{\lambda} a^{*}+(1-\bar{\lambda}) \hat{a}=\frac{4}{19} \cdot\left(\frac{1}{4}\right)+\frac{15}{19} \cdot\left(-\frac{5}{3}\right)=-\frac{24}{19}
$$

and finally $\left(a^{\prime}, b^{*}\left(a^{\prime}\right)\right)=\left(-\frac{24}{19}, \frac{23}{19}\right)$. It is easy to see that

$$
f_{\text {hor }}\left(a^{\prime}, b^{*}\left(a^{\prime}\right)\right)=f_{v e r}\left(a^{*}, b^{*}\right)
$$

Now we can provide the whole solution path of the rectangular problem, it is shown in Figure IV.2.6. For $s \in\left[0, \frac{1}{4}\right)$ it looks like the solution path of the vertical problem. Then it stays in $\left(\frac{1}{4}, \frac{23}{4}\right)$ until $s=a^{\prime}$ where it switches to the solution path of the horizontal problem. The solution path of the rectangular problem and the one of the horizontal problem are identical for $s \in\left(\left|a^{\prime}\right|, \hat{a}\right)$.

Figure IV.2.7 shows the graph of $f$ where $f$ is the following function:

$$
\begin{aligned}
f: \mathbb{R} & \rightarrow \mathbb{R} \\
s & \mapsto \min _{b \in \mathbb{R}} f_{l_{1}}(s, b) .
\end{aligned}
$$

Finally, Figure IV.2.8 shows the objective function values of the optimal solutions of $\left(P_{l_{1}}(s)\right)$ denoted by $z^{*}(s)$. This visualizes once more that the solution path gets stuck in $\left(a^{*}, b^{*}\right)$ until s reaches $a^{\prime}$ and then jumps to the horizontal solution path until it reaches the optimal solution $(\hat{a}, \hat{b})$. From there on the value of the objective function is of course constant for all $\left(P_{l_{1}}(s)\right)$. 


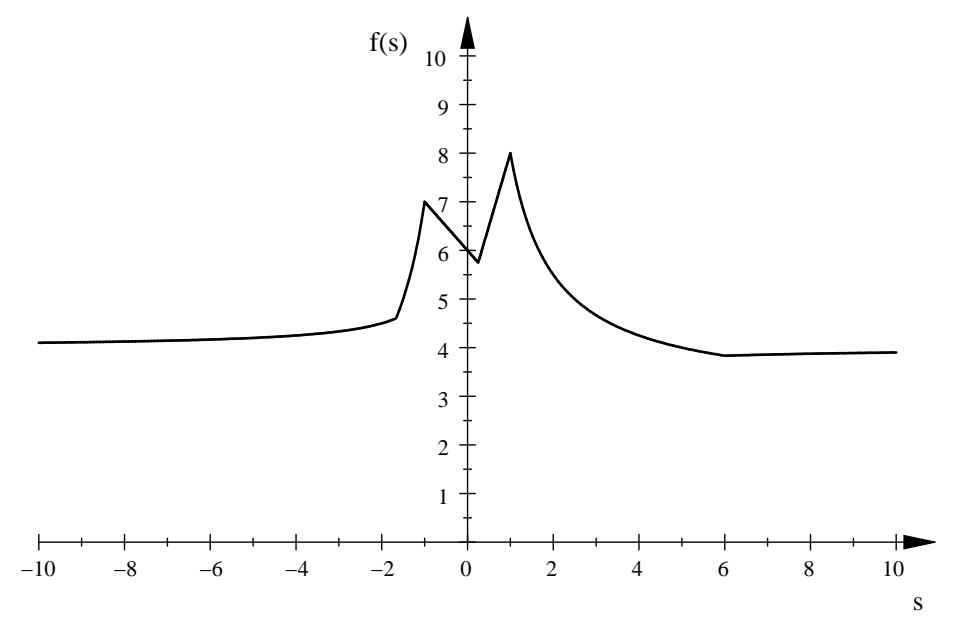

Figure IV.2.7. Graph of the function $f$ as defined in Example IV.2.4.

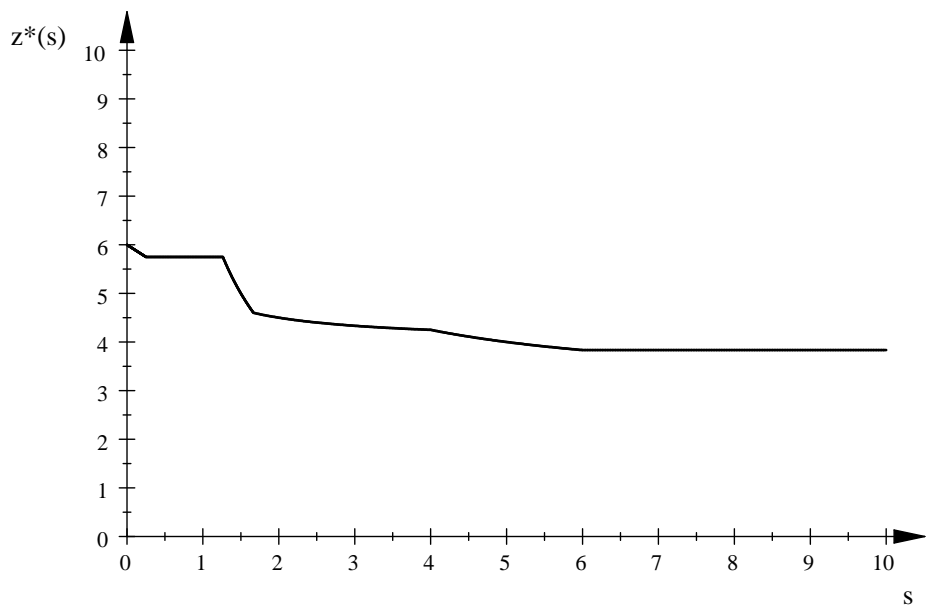

Figure IV.2.8. Function values of the optimal solutions of $\left(P_{l_{1}}(s)\right)$ with existing facilities as in Example IV.2.4 or Example IV.2.3, respectively. 


\section{Chapter V}

\section{Locating hyperplanes with a restriction on the slope using vertical distance}

In this chapter we want to examine the problem of locating a hyperplane with a restriction on the slope using the vertical distance. Obviously, locating lines with a restriction on the slope using the vertical distance is a special case of this problem since the usual lines in $\mathbb{R}^{2}$ are the hyperplanes in $\mathbb{R}^{2}$. Therefore we will see that there are a lot of parallels between this chapter and Chapter I. However, it is more difficult to guarantee the existence of an optimal trajectory which is continuous in the higher dimensional case than it was in the case of locating lines. We will present how the existence of such a trajectory can be shown and how it can be constructed.

In order to get there we will start with introducing the definition of the vertical distance between a point and a hyperplane as well as summarizing known results for the problem of locating a hyperplane using vertical distance without a restriction. 


\section{V.1 The problem of locating hyperplanes using vertical distance}

In this section we want to summarize some important results connected to hyperplane location with vertical distance. As in Section I.1, we mainly focus on results out of [Sch99]. Most of the results explicitly stated in [Sch99] are for the problem of locating a hyperplane using horizontal distance but it can be easily seen by rotation that the most important ones hold for the problem with vertical distance as well.

Remember that we are given a set of existing facilities

$$
\mathcal{E}=\left\{\left(x_{11}, \ldots, x_{1 k}, y_{1}\right), \ldots,\left(x_{n 1}, \ldots, x_{n k}, y_{n}\right)\right\} \subseteq \mathbb{R}^{k+1}
$$

and we want to locate a hyperplane $H$ in such a way that the sum of vertical distances between the hyperplane and the existing facilities is minimized. We already stated in Section 0.2 that a non-vertical hyperplane $H$ can be written as $H_{a, b}$ with $a \in \mathbb{R}^{k}$ and $b \in \mathbb{R}$, namely

$$
H_{a, b}=\left\{\left(x_{1}, \ldots, x_{k}, y\right) \in \mathbb{R}^{k+1}: y=a_{1} x_{1}+\ldots+a_{k} x_{k}+b\right\} .
$$

The vertical distance between a point $x:=\left(x_{1}, \ldots, x_{k}, y\right) \in \mathbb{R}^{k+1}$ and a hyperplane $H$ is given by

$$
d_{v e r}(x, H)=\left\{\begin{array}{l}
\left|y-\left(x_{1} \cdot a_{1}+\ldots+x_{k} \cdot a_{k}+b\right)\right| \text { if } H=H_{a, b} \text { is non-vertical } \\
0 \text { if } H \text { is vertical and } x \in H \\
\infty \text { if } H \text { is vertical and } x \notin H
\end{array}\right.
$$

Therefore the hyperplane location problem with vertical distance is of the form

$$
\min \sum_{i=1}^{n} d_{v e r}\left(\left(x_{i 1}, \ldots, x_{i k}, y_{i}\right), H\right)
$$

From now on for the rest of this chapter we assume that $\mathcal{E}$ contains at least $k+2$ 
affinely independent points. If this is not the case, the problem is trivial since all median hyperplanes $H$ contain the whole set $\mathcal{E}$ of existing facilities.

As we already mentioned in Section 0.2, we only have to investigate non-vertical hyperplanes in this chapter. The reason is that a vertical hyperplane $H$ cannot be optimal to $\left(P_{v e r}\right)$ unless all existing facilities are contained in $H$. Since we assumed that $\mathcal{E}$ contains at least $k+2$ affinely independent points this cannot be the case and we can neglect vertical hyperplanes for the rest of this chapter.

We can now reformulate our problem $\left(P_{v e r}\right)$ as

$$
\begin{aligned}
& \min f_{v e r}\left(a_{1}, \ldots, a_{k}, b\right) \\
= & \min \sum_{i=1}^{n} d_{v e r}\left(\left(x_{i 1}, \ldots, x_{i k}, y_{i}\right), H_{a_{1}, \ldots, a_{k}, b}\right) \\
= & \min \sum_{i=1}^{n}\left|y_{i}-\left(x_{i 1} a_{1}+\ldots+x_{i_{k}} a_{k}+b\right)\right| \\
= & \min \|y-(X a+b e)\|_{1}
\end{aligned}
$$

with $X \in \mathbb{R}^{n \times k}$ being the matrix

$$
X:=\left(\begin{array}{cccc}
x_{11} & x_{12} & \ldots & x_{1 k} \\
x_{21} & x_{22} & \ldots & x_{2 k} \\
\vdots & \vdots & \ddots & \vdots \\
x_{n 1} & x_{n 2} & \ldots & x_{n k}
\end{array}\right)
$$

and $e:=(1, \ldots, 1)^{t} \in \mathbb{R}^{n}$.

As in the case of locating lines, we introduce a dual interpretation of hyperplane location problems with vertical distance in order to profit from the geometric properties of $\left(P_{v e r}\right)$. The following transformation $\tilde{T}$ is a generalisation of the transformation $T$ defined in Definition I.1.2, While $T$ maps a point in $\mathbb{R}^{2}$ to a nonvertical line and vice versa, $\tilde{T}$ maps a point in $\mathbb{R}^{k+1}$ to a non-vertical hyperplane and vice versa. This transformation can also be found in [Sch99].

Definition V.1.1. We define the following transformation $\tilde{T}$ : 


\section{V.1. THE PROBLEM OF LOCATING HYPERPLANES USING}

For any point $\left(x_{1}, \ldots, x_{k}, y\right) \in \mathbb{R}^{k+1}$ let $\tilde{T}\left(x_{1}, \ldots, x_{k}, y\right)$ define a hyperplane

$$
\begin{aligned}
\tilde{T}\left(x_{1}, \ldots, x_{k}, y\right) & :=H_{-x_{1}, \ldots,-x_{k}, y} \\
& =\left\{\left(a_{1}, \ldots a_{k}, b\right): b=-x_{1} a_{1}-\ldots-x_{k} a_{k}+y\right\},
\end{aligned}
$$

and for any non-vertical hyperplane $H_{a_{1}, \ldots, a_{k}, b}$ let $\tilde{T}\left(H_{a_{1}, \ldots, a_{k}, b}\right)$ define a point

$$
\tilde{T}\left(H_{a_{1}, \ldots, a_{k}, b}\right):=\left(a_{1}, \ldots, a_{k}, b\right)
$$

The space of all transformed points and hyperplanes will be referred to as the dual space. As in the two dimensional case we have the nice property that $\tilde{T}$ preserves the vertical distances between points and hyperplanes.

Lemma V.1.1. Let $\left(x_{1}, \ldots, x_{k}, y\right) \in \mathbb{R}^{k+1}$ be a point and $H_{a_{1}, \ldots, a_{k}, b}$ a non-vertical hyperplane. Then

$$
d_{v e r}\left(\left(x_{1}, \ldots, x_{k}, y\right), H_{a_{1}, \ldots, a_{k}, b}\right)=d_{v e r}\left(\tilde{T}\left(H_{a_{1}, \ldots, a_{k}, b}\right), \tilde{T}\left(x_{1}, \ldots, x_{k}, y\right)\right) .
$$

In particular it holds that

$$
\left(x_{1}, \ldots, x_{k}, y\right) \in H_{a_{1}, \ldots, a_{k}, b} \Leftrightarrow \tilde{T}\left(H_{a_{1}, \ldots, a_{k}, b}\right) \in \tilde{T}\left(x_{1}, \ldots, x_{k}, y\right) .
$$

Proof.

$$
\begin{aligned}
d_{v e r}\left(\left(x_{1}, \ldots, x_{k}, y\right), H_{a_{1}, \ldots, a_{k}, b}\right) & =\left|y-\left(x_{1} a_{1}+\ldots+x_{k} a_{k}+b\right)\right| \\
& =\left|-y+x_{1} a_{1}+\ldots+x_{k} a_{k}+b\right| \\
& =d_{v e r}\left(\left(a_{1}, \ldots, a_{k}, b\right), H_{-x_{1}, \ldots,-x_{k}, y}\right) \\
& =d_{v e r}\left(\tilde{T}\left(H_{a_{1}, \ldots, a_{k}, b}\right), \tilde{T}\left(x_{1}, \ldots, x_{k}, y\right)\right) .
\end{aligned}
$$

This lemma directly proves the following theorem.

Theorem V.1.1. The location of a hyperplane $H_{a_{1}, \ldots, a_{k}, b}$ that minimizes the sum 


\section{LOCATING HYPERPLANES WITH A RESTRICTION ON THE SLOPE USING VERTICAL DISTANCE

of vertical distances to a given set of points

$$
\mathcal{E}=\left\{\left(x_{11}, \ldots, x_{1 k}, y_{1}\right), \ldots,\left(x_{n 1}, \ldots, x_{n k}, y_{n}\right)\right\}
$$

is equivalent to the location of a point $\left(a_{1}, \ldots, a_{k}, b\right) \in \mathbb{R}^{k+1}$ minimizing the sum of vertical distances to a given set of hyperplanes

$$
\left\{T\left(x_{11}, \ldots, x_{1 k}, y_{1}\right), \ldots, T\left(x_{n 1}, \ldots, x_{n k}, y_{n}\right)\right\}
$$

Proof. Nothing left to prove after Lemma V.1.1.

Instead of searching for a hyperplane that minimizes the sum of vertical distances to the existing facilities we can now search for a point in the dual space minimizing the sum of vertical distances to the set of dual hyperplanes corresponding to the existing facilities. For a point $\left(a_{1}, \ldots, a_{k}, b\right)$ in the dual space the objective function can be written as

$$
\begin{aligned}
f_{v e r}\left(a_{1}, \ldots, a_{k}, b\right) & =\sum_{i=1}^{n} d_{v e r}\left(\left(a_{1}, \ldots, a_{k}, b\right), H_{-x_{i 1}, \ldots,-x_{i k}, y}\right) \\
& =\sum_{i=1}^{n} d_{v e r}\left(\left(x_{i 1}, \ldots, x_{i k}, y\right), H_{a_{1}, \ldots, a_{k}, b}\right) .
\end{aligned}
$$

As in the line location case this equation shows why our notation $f_{v e r}\left(a_{1}, \ldots, a_{k}, b\right)$ for the objective function of $\left(P_{v e r}\right)$ makes perfect sense. A point $\left(a_{1}, \ldots, a_{k}, b\right)$ minimizes $f_{v e r}\left(a_{1}, \ldots, a_{k}, b\right)$ if and only if $H_{a_{1}, \ldots, a_{k}, b}$ is a hyperplane optimal for $\left(P_{\text {ver }}\right)$.

The set of transformed existing facilities $\mathcal{H}:=\left\{\tilde{T}\left(x_{i 1}, \ldots, x_{i k}, y_{i}\right): i=1 \ldots n\right\}$ partitions the dual $\mathbb{R}^{k+1}$ into cells $C \in \mathcal{C}$. On each such cell $C$, the objective function $f_{v e r}$ is linear due to the same argument as in the special case of line location: The sign of $\left|y_{i}-\left(a_{1} x_{i 1}+\ldots+a_{k} x_{i k}+b\right)\right|$ does not change on $C$. Therefore, like in the special case of line location, the problem of locating hyperplanes using vertical distance $\left(P_{v e r}\right)$ is a piecewise linear convex problem since $f_{v e r}$ is still convex in the higher dimensional case.

It is well known from optimization theory that there exists an optimal solution $\left(a_{1}^{*}, \ldots, a_{k}^{*}, b^{*}\right)$ in the dual space that is a vertex of a cell $C$. Since a vertex of 


\section{V.1. THE PROBLEM OF LOCATING HYPERPLANES USING}

a cell is an intersection of at least $k+1$ hyperplanes corresponding to affinely independent points in the initial space there exists a hyperplane minimizing the sum of vertical distances to the existing facilities passing through $k+1$ affinely independent existing facilities. We summarize this in the following lemma.

Lemma V.1.2. ([Sch99]) There exists a hyperplane minimizing the sum of vertical distances

$$
f_{v e r}\left(a_{1}, \ldots, a_{k}, b\right)=\sum_{i=1}^{n} d_{v e r}\left(\left(x_{i 1}, \ldots, x_{i k}, y_{i}\right), H_{a_{1}, \ldots, a_{k}, b}\right)
$$

and passing through $k+1$ affinely independent existing facilities.

Proof. There is nothing left to prove after the discussion above. For a more detailed proof of the corresponding statement for horizontal distances see [Sch99].

Example V.1.1. Let

$$
\mathcal{E}=\left\{\left(x_{11}, x_{12}, y_{1}\right),\left(x_{21}, x_{22}, y_{2}\right),\left(x_{31}, x_{32}, y_{3}\right),\left(x_{41}, x_{42}, y_{4}\right)\right\} \subseteq \mathbb{R}^{3}
$$

be the set of existing facilities with

$$
\begin{aligned}
& \left(x_{11}, x_{12}, y_{1}\right)=\left(-12, \frac{3}{2}, 1\right),\left(x_{21}, x_{22}, y_{2}\right)=(2,-4,-1) \\
& \left(x_{31}, x_{32}, y_{3}\right)=(-1,-2,-3) \text { and }\left(x_{41}, x_{42}, y_{4}\right)=(8,-3,4) .
\end{aligned}
$$

This is an example with $k=2$ and $n=4$. If we look at the dual space of this problem, we are given the set of hyperplanes $\mathcal{H}=\left\{H_{1}, H_{2}, H_{3}, H_{4}\right\}$ with

$$
H_{1}=H_{12,-\frac{3}{2}, 1}, H_{2}=H_{-2,4,-1}, H_{3}=H_{1,2,-3} \text { and } H_{4}=H_{-8,3,4} .
$$

Lemma V.1.2 tells us that there exists an optimal solution to the unrestricted problem $\left(P_{\text {ver }}\right)$ passing through $k+1$ affinely independent existing facilities. Therefore it is sufficient to check the objective function values of the points

$$
\begin{aligned}
& \left(a_{1}, a_{2}, b\right)_{123}=\left(-\frac{30}{23},-\frac{68}{23},-\frac{235}{23}\right),\left(a_{1}, a_{2}, b\right)_{124}=\left(\frac{51}{94}, \frac{82}{47}, \frac{230}{47}\right), \\
& \left(a_{1}, a_{2}, b\right)_{134}=\left(\frac{57}{41}, \frac{226}{41}, \frac{386}{41}\right) \text { and }\left(a_{1}, a_{2}, b\right)_{234}=\left(\frac{4}{5}, \frac{1}{5},-\frac{9}{5}\right),
\end{aligned}
$$




\section{LOCATING HYPERPLANES WITH A RESTRICTION ON THE SLOPE USING VERTICAL DISTANCE

where $\left(a_{1}, a_{2}, b\right)_{i, j, k}$ corresponds to the intersection of $H_{i}, H_{j}$ and $H_{k} \in \mathcal{H}$. It is easy to check that $\left(a_{1}, a_{2}, b\right)_{124}$ provides the best objective function value, namely $f_{\text {ver }}\left(\left(a_{1}, a_{2}, b\right)_{124}\right)=\frac{363}{94}$, and therefore $\left(a_{1}, a_{2}, b\right)_{124}$ is an optimal solution for $\left(P_{v e r}\right)$.

Finally, we want to provide a lemma which tells us that every hyperplane minimizing the sum of vertical distances to the existing facilities is pseudo-halving. Remember that we already introduced the concept of pseudo-halving lines in Section I.2. This is just a special case of the concept of pseudo-halving hyperplanes which has been introduced in [Sch99] as-well. Considering only the special case without weights or to be more precise the case with $w_{i}=1$ for all $i=1, \ldots, n$ and our notation

$$
\begin{aligned}
R\left(H_{a_{1}, \ldots, a_{k}, b}\right) & =\left\{\left(x_{i 1}, \ldots, x_{i k}, y_{i}\right) \in \mathcal{E}: y_{i}-\left(a_{1} x_{i 1}+\ldots+a_{k} x_{i k}+b\right)>0\right\} \\
L\left(H_{a_{1}, \ldots, a_{k}, b}\right) & =\left\{\left(x_{i 1}, \ldots, x_{i k}, y_{i}\right) \in \mathcal{E}: y_{i}-\left(a_{1} x_{i 1}+\ldots+a_{k} x_{i k}+b\right)<0\right\}
\end{aligned}
$$

for a hyperplane $H_{a_{1}, \ldots, a_{k}, b}$, we get the following definition.

Definition V.1.2. ([Sch99]) A hyperplane $H$ is called pseudo-halving if

$$
\begin{gathered}
\sum_{\left(x_{i 1}, \ldots, x_{i k}, y_{i}\right) \in L(H)} 1=|L(H)| \leq \frac{n}{2} \text { and } \\
\sum_{\left(x_{i 1}, \ldots, x_{i k}, y_{i}\right) \in R(H)} 1=|R(H)| \leq \frac{n}{2} .
\end{gathered}
$$

Now we can formulate the following lemma.

Lemma V.1.3. ([Sch99]) Every hyperplane $H^{*}$ minimizing

$$
f_{v e r}\left(a_{1}, \ldots, a_{k}, b\right)=\sum_{i=1}^{n} d_{v e r}\left(\left(x_{i 1}, \ldots, x_{i k}, y_{i}\right), H\right)
$$

is pseudo-halving.

Proof. We will not give a detailed proof of the lemma here. However, the proof of this lemma is more or less analogous to the proof of Theorem $\mathrm{V} .2 .2$ in Section V.2. 


\section{V.2. FIRST RESULTS ON LOCATING HYPERPLANES WITH A 112 RESTRICTION ON THE SLOPE}

Lemma V.1.3 together with Lemma V.1.2 give a finite candidate set for $\left(P_{v e r}\right)$ : Any hyperplane $H$ that passes $k+1$ affinely independent existing facilities while it is pseudo-halving is a candidate for an optimal solution to $\left(P_{v e r}\right)$.

\section{V.2 First results on locating hyperplanes with a restriction on the slope}

In Section V.1 we summarized the results for the unrestricted problem $\left(P_{\text {ver }}\right)$. As we have seen, a candidate set for the unrestricted problem of locating a hyperplane is known. We want to use the presented results and expand them to the problem we are interested in throughout this chapter, that is the location of a hyperplane that minimizes the sum of vertical distances to a set of existing facilities when there is given a restriction on the slope of the hyperplane to be located. Before we start, remember the formulation of our problem for a given $s \geq 0$ :

$$
\begin{aligned}
& \min f_{v e r}\left(a_{1}, \ldots, a_{k}, b\right)=\min \sum_{i=1}^{n}\left|y_{i}-\left(x_{i 1} a_{1}+\ldots+x_{i k} a_{k}+b\right)\right| \quad\left(P_{v e r}(s)\right) \\
& \text { s.t. }\|a\|_{1}=\left|a_{1}\right|+\ldots+\left|a_{k}\right| \leq s
\end{aligned}
$$

The main goal of this chapter is to show that there exists an optimal trajectory for our problem that is continuous in $s$. In other words, first we want to be able to solve the problem $\left(P_{v e r}(s)\right)$ for any $s \geq 0$ and next we want to show that we can select elements from the solution sets of the different problems $\left(P_{v e r}(s)\right), s \geq 0$, in such a way that the trajectory these elements describe in $\mathbb{R}^{k+1}$ is continuous. Our trajectory will start with a solution for the problem $\left(P_{\text {ver }}(0)\right)$ and finally end up in an optimal solution for the unrestricted problem $\left(P_{v e r}\right)$.

In order to solve $\left(P_{\text {ver }}(s)\right)$ for any $s \geq 0$, we want to use the dual space introduced in Section V.1 again. For simplification of our notation we denote an existing facility by $\left(x_{i}, y_{i}\right)$ instead of $\left(x_{i 1}, \ldots, x_{i k}, y_{i}\right)$. Let now $\mathcal{E}=\left\{\left(x_{i}, y_{i}\right) \in \mathbb{R}^{k+1}: i=1, \ldots, n\right\}$ be the set of existing facilities. In a similar way, we use $H_{a, b}$ short for a hyperplane $H_{a_{1}, \ldots, a_{k}, b}$. Now we want to locate a hyperplane $H_{a, b}$ such that the sum of vertical 


\section{LOCATING HYPERPLANES WITH A RESTRICTION ON THE SLOPE USING VERTICAL DISTANCE

distances to the existing facilities $\left(x_{i}, y_{i}\right) \in \mathcal{E}$ is minimized while $\|a\|_{1} \leq s$ holds.

In the dual space, this corresponds to the following problem: Given a set of hyperplanes $\mathcal{H}=\left\{H_{-x_{1}, y_{1}}, \ldots, H_{-x_{n}, y_{n}}\right\}$ where $-x_{i}$ means $\left(-x_{i 1}, \ldots,-x_{i k}\right)$ locate a point $(a, b) \in \mathbb{R}^{k+1}$ that minimizes the sum of vertical distances to the existing hyperplanes such that $\|a\|_{1} \leq s$ holds.

As in the case of line location, the restriction on the $a$-vector leads to a different cell structure in the dual space compared to the unrestricted case. As a matter of fact, we have to add several hyperplanes to the set of existing hyperplanes $\mathcal{H}$. These are the hyperplanes described by all possible equations

$$
\delta_{1} a_{1}+\ldots+\delta_{k} a_{k}=s
$$

with $\delta_{j}$ being +1 or -1 . In other words, we have to add $2^{k}$ hyperplanes of the form $H=\left\{(a, b): \delta_{1} a_{1}+\ldots+\delta_{k} a_{k}=s\right\}$ to $\mathcal{H}$ which is in line with adding just 2 lines in the case of line location.

They define a kind of a "tube" around the $b$-axis in the dual $\mathbb{R}^{k+1}$ in which an optimal solution to $\left(P_{v e r}(s)\right)$ has to lie. We denote the set of additional hyperplanes by $\mathcal{H}^{\prime}$.

In addition to the hyperplanes coming from the restriction on the $a$-vector we want to consider the $k$ different hyperplanes of the form

$$
H_{j}=\left\{(a, b) \in \mathbb{R}^{k+1}: a_{j}=0\right\}
$$

By $\mathcal{H}^{\prime \prime}$ we denote the set of these hyperplanes. This is new in comparison to the case of line location. We will see later on that this set of hyperplanes is very useful for the geometrical interpretation of the optimal trajectory.

The hyperplanes in $\mathcal{H} \cup \mathcal{H}^{\prime} \cup \mathcal{H}^{\prime \prime}$ again induce a cell structure on the dual space, in other words they partition the dual $\mathbb{R}^{k+1}$ into cells $\tilde{C} \in \tilde{\mathcal{C}}$. Since this new partition is a subdivision of the partition induced by $\mathcal{H}$ alone, the sign of $y_{i}-\left(a_{1} x_{i 1}+\ldots+\right.$ $\left.a_{k} x_{i k}, b\right)$ still does not change on each cell $\tilde{C}$ for all $i$, therefore $\left(P_{v e r}(s)\right)$ is still a piecewise linear convex problem. This results in the fact that again there exists an optimal solution $\left(a^{*}, b^{*}\right) \in \mathbb{R}^{k+1}$ that is a vertex of a cell $\tilde{C}$. Unfortunately this vertex does no longer have to be an intersection of $k+1$ hyperplanes corresponding 


\section{V.2. FIRST RESULTS ON LOCATING HYPERPLANES WITH A 114 RESTRICTION ON THE SLOPE}

to $k+1$ affinely independent existing facilities. It can actually happen that this vertex is an intersection of at least $k$ hyperplanes of the set $\mathcal{H}^{\prime} \cup \mathcal{H}^{\prime \prime}$ and only one single hyperplane $H_{-x_{i}, y_{i}} \in \mathcal{H}$.

Note that it may happen that $k+1$ or more hyperplanes of the set $\mathcal{H}^{\prime} \cup \mathcal{H}^{\prime \prime}$ intersect but this intersection cannot correspond to a vertex of a cell. No matter which hyperplanes of this set intersect, if $(\bar{a}, \bar{b})$ is in the intersection so is $(\bar{a}, b)$ for all $b \in \mathbb{R}$. Therefore an intersection of hyperplanes of the set $\mathcal{H}^{\prime} \cup \mathcal{H}^{\prime \prime}$ is at least one-dimensional. This assures that one of the hyperplanes corresponding to an existing facility is needed to describe a vertex of a cell $\tilde{C} \in \tilde{\mathcal{C}}$.

Therefore all we can say about optimal solutions of $\left(P_{v e r}(s)\right)$ so far is that there always exists an optimal hyperplane in the initial space that passes trough at least one of the existing facilities. This is the statement of our first theorem of this chapter.

Theorem V.2.1. There exists a hyperplane optimizing $\left(P_{\text {ver }}(s)\right)$ that passes through at least one of the given facilities.

Proof. Following the argumentation above yields the proof of this theorem.

Next we can prove that still any optimal hyperplane is pseudo-halving in the restricted case. The corresponding statement for the unrestricted case was given in Lemma V.1.3 of the previous section. There you can also look up the definition of a pseudo-halving hyperplane if needed. The subsequent theorem is analogous to Theorem I.2.2 in Section I.2. As in the case of line location, we need a proposition first that is the generalization of Proposition I.2.1 to the case of hyperplanes. For a definition of a median of a set of numbers, see Definition I.2.3 in Section I.2.

Proposition V.2.1. Let $\mathcal{E}=\left\{\left(x_{i 1}, \ldots, x_{i k}, y_{i}\right) \in \mathbb{R}^{k+1}, i=1 \ldots n\right\}$ be a set of existing facilities and let $\left(a_{1}^{*}, \ldots, a_{k}^{*}, b^{*}\right) \in \mathbb{R}^{k+1}$ be an optimal solution for $\left(P_{\text {ver }}(s)\right)$. Define a real-valued function of a real variable

$$
\bar{f}: \mathbb{R} \rightarrow \mathbb{R}
$$

by

$$
b \mapsto \sum_{i=1}^{n}\left|a_{1}^{*} x_{i 1}+\ldots+a_{k}^{*} x_{i k}-y_{i}+b\right|
$$




\section{LOCATING HYPERPLANES WITH A RESTRICTION ON THE SLOPE USING VERTICAL DISTANCE

The value $b^{*}$ minimizes $\bar{f} \Leftrightarrow b^{*} \in \operatorname{median}\left\{\left(y_{i}-\left(a_{1}^{*} x_{i 1}+\ldots+a_{k}^{*} x_{i k}\right), 1\right): i=1 \ldots n\right\}$.

Proof. " $\Rightarrow$ " The function $\bar{f}$ is piecewise linear and convex.

Suppose $b^{*}$ optimizes $\bar{f}$ but $b^{*} \notin$ median $\left\{\left(y_{i}-\left(a_{1}^{*} x_{i 1}+\ldots+a_{k}^{*} x_{i k}\right), 1\right)\right\}$. Without loss of generality we can suppose that

$$
\sum_{i: y_{i}-\left(a_{1}^{*} x_{i 1}+\ldots+a_{k}^{*} x_{i k}\right)<b^{*}} 1>\frac{n}{2}
$$

Now there exists an $\epsilon>0$ so that

$$
\begin{aligned}
& \left\{i: y_{i}-\left(a_{1}^{*} x_{i 1}+\ldots+a_{k}^{*} x_{i k}\right)<b^{*}\right\}=\left\{i: y_{i}-\left(a_{1}^{*} x_{i 1}+\ldots+a_{k}^{*} x_{i k}\right)<b^{*}-\epsilon\right\} \\
& \left\{i: y_{i}-\left(a_{1}^{*} x_{i 1}+\ldots+a_{k}^{*} x_{i k}\right) \geq b^{*}\right\}=\left\{i: y_{i}-\left(a_{1}^{*} x_{i 1}+\ldots+a_{k}^{*} x_{i k}\right) \geq b^{*}-\epsilon\right\}
\end{aligned}
$$

But then it holds that

$$
\begin{aligned}
& \bar{f}\left(b^{*}-\epsilon\right)= \sum_{i: y_{i}-\left(a_{1}^{*} x_{i 1}+\ldots+a_{k}^{*} x_{i k}\right)<b^{*}}\left(b^{*}-\epsilon-\left(y_{i}-\left(a_{1}^{*} x_{i 1}+\ldots+a_{k}^{*} x_{i k}\right)\right)\right) \\
&+\sum_{i: y_{i}-\left(a_{1}^{*} x_{i 1}+\ldots+a_{k}^{*} x_{i k}\right) \geq b^{*}}\left(\left(y_{i}-\left(a_{1}^{*} x_{i 1}+\ldots+a_{k}^{*} x_{i k}\right)\right)-b^{*}+\epsilon\right) \\
&= \sum_{i: y_{i}-\left(a_{1}^{*} x_{i 1}+\ldots+a_{k}^{*} x_{i k}\right)<b^{*}}\left(b^{*}-\left(y_{i}-\left(a_{1}^{*} x_{i 1}+\ldots+a_{k}^{*} x_{i k}\right)\right)\right) \\
&+\sum_{i: y_{i}-\left(a_{1}^{*} x_{i 1}+\ldots+a_{k}^{*} x_{i k}\right) \geq b^{*}}\left(\left(y_{i}-\left(a_{1}^{*} x_{i 1}+\ldots+a_{k}^{*} x_{i k}\right)\right)-b^{*}\right) \\
&+\epsilon\left(\sum_{i: y_{i}-\left(a_{1}^{*} x_{i 1}+\ldots+a_{k}^{*} x_{i k}\right) \geq b^{*}} 1-\sum_{i: y_{i}-\left(a_{1}^{*} x_{i 1}+\ldots+a_{k}^{*} x_{i k}\right)<b^{*}}\right) \\
&<\bar{f}\left(b^{*}\right)+\epsilon\left(\frac{n}{2}-\frac{n}{2}\right) \\
&=\bar{f}\left(b^{*}\right),
\end{aligned}
$$

which contradicts the assumption of optimality of $b^{*}$.

" $\Leftarrow$ " Let $b^{*} \in$ median $\left\{\left(y_{i}-\left(a_{1}^{*} x_{i 1}+\ldots+a_{k}^{*} x_{i k}\right), 1\right)\right\}$. For $\epsilon>0$ small enough it can be shown similarly to the proof of the other direction that

$$
\bar{f}\left(b^{*}-\epsilon\right) \geq \bar{f}\left(b^{*}\right) \text { and } \bar{f}\left(b^{*}+\epsilon\right) \geq \bar{f}\left(b^{*}\right) .
$$




\section{V.2. FIRST RESULTS ON LOCATING HYPERPLANES WITH A 116 RESTRICTION ON THE SLOPE}

By convexity of $\bar{f}$ the optimality of $b^{*}$ follows.

This is all we need to prove the following theorem.

Theorem V.2.2. Any hyperplane $H^{*}$ which minimizes

$$
\begin{aligned}
& f_{v e r}(a, b)=\sum_{i=1}^{n} d_{v e r}\left(\left(x_{i}, y_{i}\right), H_{a, b}\right) \\
& \text { s.t. }\|a\|_{1} \leq s
\end{aligned}
$$

is pseudo-halving.

Proof. The proof of this theorem works analogous to the proof of Theorem I.2.2 in Section I.2.

Let $H^{*}=H_{a_{1}^{*}, \ldots, a_{k}^{*}, b^{*}}$ be an optimal solution to $\left(P_{v e r}\right)$, therefore in particular it holds that $\left\|a^{*}\right\|_{1} \leq s$.

Keep $a^{*} \in \mathbb{R}^{k}$ fixed and look at the function

$$
\begin{aligned}
\bar{f}(b) & =\sum_{i=1}^{n} d_{v e r}\left(\left(x_{i}, y_{i}\right), H_{a^{*}, b}\right) \\
& =\sum_{i=1}^{n}\left|y_{i}-\left(a_{1}^{*} x_{i 1}+\ldots+a_{k}^{*} x_{i k}+b\right)\right|
\end{aligned}
$$

which is a function of a real variable $b$. We can now look at the minimization problem

$$
\min \bar{f}(b) \text {. }
$$

Since $H^{*}$ is optimal for $\left(P_{v e r}(s)\right)$, one optimal solution to $(P)$ is $b^{*}$. But this means that $b^{*}$ is a median of the set $\left\{\left(y_{i}-\left(a_{1}^{*} x_{i 1}+\ldots+a_{k}^{*} x_{i k} ; 1\right): i=1, \ldots, n\right\}\right.$ due to Proposition V.2.1. In other words, it holds that

$$
\begin{aligned}
\sum_{i: y_{i}>a_{1}^{*} x_{i 1}+\ldots+a_{k}^{*} x_{i k}+b} 1=\left|R\left(H^{*}\right)\right| & \leq \frac{n}{2} \text { as well as } \\
\sum_{i: y_{i}<a_{1}^{*} x_{i 1}+\ldots+a_{k}^{*} x_{i k}+b} 1=\left|L\left(H^{*}\right)\right| & \leq \frac{n}{2}
\end{aligned}
$$

by definition of a median of a set of numbers.

This already tells us that $H^{*}$ is a pseudo-halving hyperplane and we are done. 


\section{LOCATING HYPERPLANES WITH A RESTRICTION ON THE SLOPE USING VERTICAL DISTANCE

The fact that an optimal hyperplane for $\left(P_{v e r}(s)\right), s \geq 0$, has to be pseudo-halving leads to the following corollary.

Corollary V.2.1. The set $\left\{b \in \mathbb{R}:(a, b)\right.$ is optimal to $\left.\left(P_{\text {ver }}\right)\right\}$ is bounded for any $s \geq 0$.

Up to now, we know that any optimal solution to $\left(P_{v e r}(s)\right)$ is a pseudo-halving hyperplane and that there exists an optimal hyperplane that corresponds to a vertex of a cell in the dual space. Before we go on, we want to introduce some notation. From now on for the rest of this chapter, we denote the sets of optimal solutions of $\left(P_{v e r}\right)$ and $\left(P_{v e r}(s)\right)$ by $M_{v e r}^{\text {opt }} \subseteq \mathbb{R}^{k+1}$ and $M_{v e r}^{\text {opt }}(s) \subseteq \mathbb{R}^{k+1}$, respectively, as we did in the case of line location.

Note that once we have reached an optimal solution for the unrestricted problem $\left(P_{v e r}\right)$, this solution will be optimal for all following restricted problems. Define $s^{*}:=\min \left\{\|a\|_{1}:(a, b) \in M_{v e r}^{o p t}\right\}$.

Lemma V.2.1. Let $\left(a^{*}, b^{*}\right) \in M_{v e r}^{\text {opt }}$ with $\left\|a^{*}\right\|_{1}=s^{*}$. Then $\left(a^{*}, b^{*}\right) \in M_{\text {ver }}^{\text {opt }}(s)$ for all $s \geq s^{*}$.

Proof. Since $\left(a^{*}, b^{*}\right)$ is an optimal solution for the unrestricted problem and it is feasible for $\left(P_{v e r}(s)\right)$ for all $s \geq s^{*},\left(a^{*}, b^{*}\right)$ obviously is an element of $M_{v e r}^{\text {opt }}(s)$ for all $s \geq s^{*}$.

Next we want to show that for any optimal hyperplane $H_{a, b}$ of a problem $\left(P_{v e r}(s)\right)$ such that $0 \leq s \leq s^{*}$ it must hold that $\|a\|_{1}=s$. This is due to the convexity of our problem as we will show in the subsequent theorem.

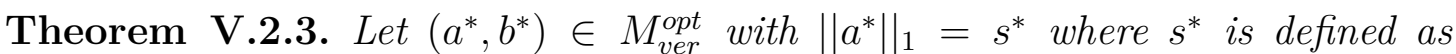
$s^{*}:=\min \left\{\|a\|_{1}:(a, b) \in M_{\text {ver }}^{\text {opt }}\right\}$. If $s \in\left[0, s^{*}\right]$ then for all $(a, b) \in M_{\text {ver }}^{\text {opt }}(s)$ it holds that $\|a\|_{1}=s$.

Proof. If $s^{*}=0$ that would mean that there exists an optimal solution for $\left(P_{v e r}\right)$ satisfying $a=0$. In this case, our statement is true.

Assume that $s^{*}>0$. Let $s \in\left[0, s^{*}\right]$ and assume that there exists a $(a, b) \in M_{\text {ver }}^{\text {opt }}(s)$ such that $\|a\|_{1}<s$. 


\section{V.2. FIRST RESULTS ON LOCATING HYPERPLANES WITH A 118 RESTRICTION ON THE SLOPE}

If $s=s^{*}$, then obviously it holds that $(a, b) \in M_{v e r}^{\text {opt }}$ since $M_{v e r}^{\text {opt }}\left(s^{*}\right) \subseteq M_{v e r}^{\text {opt }}$. But then it holds that $\|a\|_{1}<\left\|a^{*}\right\|_{1}$ and $(a, b) \in M_{v e r}^{\text {opt }}$ which is a contradiction to the definition of $s^{*}$.

Let now finally be $s \in\left[0, s^{*}\right)$. Then $\left\|a^{\prime}\right\|_{1}>s$ for all $\left(a^{\prime}, b^{\prime}\right) \in M_{\text {ver }}^{\text {opt }}$. In particular it holds that $\left\|a^{*}\right\|_{1}>s$ and it is

$$
f_{v e r}(a, b)>f_{v e r}\left(a^{*}, b^{*}\right)
$$

since otherwise $(a, b) \in M_{v e r}^{\text {opt }}$ which leads to the same contradiction as we had in the case above.

Since we assumed $\|a\|_{1}<s$ and $\left\|a^{*}\right\|_{1}>s$, there exists a $\lambda \in(0,1)$ such that

$$
\left\|\lambda a+(1-\lambda) a^{*}\right\|_{1}=s .
$$

Then $\left(\lambda a+(1-\lambda) a^{*}, \lambda b+(1-\lambda) b^{*}\right)$ is feasible for $\left(P_{v e r}(s)\right)$ and

$$
\begin{aligned}
f_{v e r}\left(\lambda a+(1-\lambda) a^{*}, \lambda b+(1-\lambda) b^{*}\right) & \leq \lambda f_{v e r}(a, b)+(1-\lambda) f_{v e r}\left(a^{*}, b^{*}\right) \\
& <\lambda f_{v e r}(a, b)+(1-\lambda) f_{v e r}(a, b) \\
& =f_{v e r}(a, b)
\end{aligned}
$$

where the first inequality holds due to the convexity of $f_{v e r}$ and the second one due to $(\star)$. But this is a contradiction to $(a, b) \in M_{v e r}^{\text {opt }}(s)$ and the theorem follows.

The preceding theorem tells us that an optimal solution to $\left(P_{\text {ver }}(s)\right), 0 \leq s \leq s^{*}$, has to lie on the boundary of the "tube" around the $b$-axis defined by the set of hyperplanes we denoted by $\mathcal{H}^{\prime}$. This is a special case of the boundary theorem we already mentioned on page 91 .

Summarizing our results so far, it is sufficient to consider all hyperplanes $H_{a, b}$ corresponding to a vertex $(a, b)$ of a cell $\tilde{C} \in \tilde{\mathcal{C}}$ in the dual space which is pseudohalving and satisfies $\|a\|_{1}=s$ as candidates for an optimal solution to $\left(P_{v e r}(s)\right)$. 
V.3 The existence of a continuous optimal trajectory

For the rest of this chapter, our goal will be to show that there exists an optimal trajectory for our problem $\left(P_{v e r}(s)\right)$ which is continuous and to give an idea of how to construct it. First we will see that it is possible to find $\left(a^{*}(s), b^{*}(s)\right) \in M_{v e r}^{\text {opt }}(s)$ in such a way that $a_{j}^{*}(s)$ is piecewise linear in $s$ for all $j \in\{1, \ldots, k\}$ as well as $b^{*}(s)$ is piecewise linear in $s$. We can use this fact to show that there exists a continuous optimal trajectory via the well-known Michael selection theorem. This theorem was first introduced in [Mic56]. In order to do so, we will have to learn a bit about so-called set-valued maps. Note that in literature, there are different expressions to denote the same concept of set-valued maps, e.g. multivalued functions or pointto-set mappings. A nice introduction to this topic can be found in [AF90]. As soon as we have shown the existence of such a continuous optimal trajectory, we want to present a method of how to construct one.

From now on we assume that there exists no optimal solution $\left(a^{*}, b^{*}\right) \in M_{v e r}^{\text {opt }}$ of $\left(P_{v e r}\right)$ such that $\left\|a^{*}\right\|_{1}=0$. This is no restriction because if there exists such a solution $\left(a^{*}, b^{*}\right)$ it is of course optimal for $\left(P_{v e r}(s)\right)$ for any $s \geq 0$ (see Lemma $\overline{\nabla .2 .1}$, and therefore an optimal trajectory is given by the single point $\left(a^{*}, b^{*}\right)$.

In order to deal with set-valued maps, we first have to learn how a set-valued map looks like. Therefore we start off with the definition of such a map.

Definition V.3.1. ([AF90]) Let $X$ and $Y$ be topological spaces. A set-valued map F

$$
F: X \rightarrow 2^{Y}
$$

where $2^{Y}$ is the power set of $Y$ is characterized by its graph $G r a p h(F)$, the subset of the product space $X \times Y$ defined by

$$
\operatorname{Graph}(F):=\{(x, y) \in X \times Y: y \in F(x)\}
$$

$F(x)$ often is referred to as the image or the value of $F$ at $x$. A set-valued map is said to be non-trivial if its graph is not empty, i.e., if there exists at least an element $x \in X$ such that $F(x)$ is not empty. 
$F$ is called strict if all images $F(x)$ are not empty.

Note that the function

$$
\begin{aligned}
F^{*}: \mathbb{R}_{0}^{+} & \rightarrow 2^{\mathbb{R}^{k+1}} \\
s & \mapsto M_{\text {ver }}^{\text {opt }}(s)
\end{aligned}
$$

is a strict set-valued map since $\mathbb{R}$ and $\mathbb{R}^{k+1}$ are obviously both topological spaces and $M_{v e r}^{o p t}(s)$ is not empty for all $s \geq 0$ since we know that there always exists an optimal hyperplane (see Theorem V.2.1). We therefore can interpret the set of optimal solutions of $\left(P_{v e r}(s)\right)$ as the image of $F^{*}$ while $F^{*}$ is characterized by its graph $\operatorname{Graph}\left(F^{*}\right)=\left\{(s,(a, b)) \in \mathbb{R} \times \mathbb{R}^{k+1}:(a, b) \in M_{\text {ver }}^{\text {opt }}(s)\right\}$, the set of all tuples of $s$ and an optimal solution for $\left(P_{v e r}(s)\right)$.

What we want to find out is if there is a way to choose one element $(a(s), b(s)) \in$ $M_{\text {ver }}^{\text {opt }}(s)$ out of each set $M_{\text {ver }}^{\text {opt }}(s), s \geq 0$, in such a way that the function $t: \mathbb{R} \rightarrow \mathbb{R}^{k+1}$ defined through

$$
\begin{aligned}
t: \mathbb{R}_{0}^{+} & \rightarrow \mathbb{R}^{k+1} \\
s & \mapsto(a(s), b(s))
\end{aligned}
$$

is continuous in $s$. Note that the function $t$ defined through our choices of $(a(s), b(s)) \in M_{\text {ver }}^{\text {opt }}(s), s \geq 0$, now maps a $s \geq 0$ to one single point $(a(s), b(s))$ in $\mathbb{R}^{k+1}$ which corresponds to a hyperplane $H_{a(s), b(s)}$ optimal for $\left(P_{v e r}(s)\right)$. The fact that we call this function $t$ already gives a hint that this function in fact gives us the continuous optimal trajectory we are looking for if we choose the $(a(s), b(s))$ in the right way. Independent from the choice of $(a(s), b(s))$ it gives us an optimal trajectory in any case. Such a continuous function $t$ corresponds to what is called a selection in [Mic56].

Definition V.3.2. ([Mic56]) Let $X$ and $Y$ be topological spaces and let $F: X \rightarrow$ $2^{Y}$ be a set-valued function. Then a selection of $F$ is a continuous $f: X \rightarrow Y$ such that

$$
f(x) \in F(x)
$$

for every $x \in X$. 


\section{LOCATING HYPERPLANES WITH A RESTRICTION ON THE SLOPE USING VERTICAL DISTANCE

Therefore in terms of set-valued analysis, we are interested in finding a selection $t$ of the set-valued mapping $F^{*}$. To be more precise, we first want to prove that there exists such a selection. As we already mentioned at the beginning of this section, we want to apply the Michael selection theorem in order to prove the existence. We will now state the theorem and afterwards we will discuss what features we have to prove for our set-valued function $F^{*}$ in order to apply it. If you are interested in a proof of the theorem, look up Theorem 3.2" in [Mic56].

Theorem V.3.1 (Michael selection theorem). Let $X$ be a paracompact $T_{1}$-space and let $Y$ be a Banach space. If $F: X \rightarrow 2^{Y}$ is a lower semicontinuous set-valued map with non-empty convex closed values then there exists a selection $f: X \rightarrow Y$ of $F$.

In order to apply the Michael selection theorem we have to check several things. To be able to do so, we need a short excursion to the field of general topology. We will not go into too much detail here, but for completeness of our work we want to show briefly that it is sensitive to apply the Michael selection theorem to our problem. First of all we have to check whether $\mathbb{R}$ is a paracompact $T_{1}$-space. Let us therefore recall what characteristics define a $T_{1}$-space and a paracompact space, respectively. These definitions can be found in numerous books about topology, e.g. [Kel55].

Definition V.3.3. A topological space is a $T_{1}$-space iff each set which consists of a single point is closed.

Fortunately, we have to deal with $(\mathbb{R},|\cdot|)$ which is a space that has a lot of nice features. It is easy to see that $(\mathbb{R},|\cdot|)$ is indeed a $T_{1}$-space once you know the definition of such a topological space.

In order to give the definition of a paracompact space in the terms of [Kel55], we first have to define a regular space.

Definition V.3.4. A topological space is regular iff for each point $x$ and each neighbourhood $U$ of $x$ there is a closed neighbourhood $V$ such that $V \subset U$.

Definition V.3.5. A topological space is paracompact iff it is regular and each open cover has an open locally finite refinement. 


\section{V.3. THE EXISTENCE OF A CONTINUOUS OPTIMAL}

As we already mentioned above, we do not want to go into detail here, but it is stated in [Sto48] that any metric space is paracompact. Therefore $(\mathbb{R},|\cdot|)$ is of course paracompact as a metric space.

Now we know that $\mathbb{R}$ fulfils everything we need it to fulfil in order to be able to apply the Michael selection theorem. Next, it is well-known that $\mathbb{R}^{k+1}$ is a Banach space, therefore we will not get any problem here.

We already mentioned that $F^{*}$ is a strict set-valued map which means that it has non-empty values. What we need to show is that the values of $F^{*}$ are convex and closed. In other words, we have to show that $M_{v e r}^{\text {opt }}(s)$ is convex and closed for any $s \geq 0$.

It is well-known in optimization theory that the set of optimal solutions of a convex problem is a convex set. Since $M_{v e r}^{o p t}(s)$ is the set of optimal solutions of the convex problem $\left(P_{v e r}(s)\right)$ for any $s$, we know that $M_{v e r}^{\text {opt }}(s)$ is a convex set. Remember that

$$
\begin{aligned}
M_{v e r}^{\text {opt }}(s)=\{( & (a, b) \in \mathbb{R}^{k+1}:\|a\|_{1} \leq s \text { and } f_{\text {ver }}(a, b) \leq f_{\text {ver }}\left(a^{\prime}, b^{\prime}\right) \forall\left(a^{\prime}, b^{\prime}\right) \\
& \text { satisfying } \left.\left\|a^{\prime}\right\|_{1} \leq s\right\} .
\end{aligned}
$$

We know that $b$ is bounded for $(a, b) \in M_{\text {ver }}^{\text {opt }}(s)$ due to the pseudo-halving property of an optimal hyperplane (see Corollary $\overline{\mathrm{V} .2 .1}$ ). Moreover, as the set of optimal solutions of $\left(P_{\text {ver }}(s)\right), M_{\text {ver }}^{\text {opt }}(s)$ is either a vertex of a cell $\tilde{C}$, a facet of a cell $\tilde{C}$ or a whole cell $\tilde{C} \in \tilde{\mathcal{C}}$, where $\tilde{\mathcal{C}}$ are the cells defined through the set of hyperplanes corresponding to existing facilities together with the set of hyperplanes corresponding to the restriction on the slope in the dual space and the hyperplanes $H_{j}=\left\{(a, b) \in \mathbb{R}^{k+1}: a_{j}=0\right\}$ with $j=1, \ldots, k$, i.e. $\mathcal{H} \cup \mathcal{H}^{\prime} \cup \mathcal{H}^{\prime \prime}$ if we put it as in Section V.2. Therefore $M_{\text {ver }}^{\text {opt }}(s)$ is indeed a closed set for any $s \geq 0$.

Putting it all together, we have that $\mathbb{R}$ is a paracompact $T_{1}$-space, $\mathbb{R}^{k+1}$ is a Banach space and $F^{*}: \mathbb{R}_{0}^{+} \rightarrow 2^{\mathbb{R}^{k+1}}$ is a set-valued map with non-empty convex closed values. What we do not have up to now is that $F^{*}$ is lower semicontinuous. Unfortunately, this is not trivial to prove directly by just applying the definition of a lower semicontinuous set-valued function which we will get to know soon. This is why we take a detour and show that there exist functions $a, b$ such that 


\section{LOCATING HYPERPLANES WITH A RESTRICTION ON THE SLOPE USING VERTICAL DISTANCE

$(a(s), b(s)) \in M_{v e r}^{\text {opt }}(s)$ for all $s \geq 0$ and furthermore $a_{j}(s)$ is piecewise linear in $s$ for all $j=1 \ldots k$ as well as $b(s)$ is piecewise linear in $s$. That will help us to show that $F^{*}$ is indeed lower semicontinuous.

Let us start with the definitions of lower semicontinuity, upper semicontinuity and continuity of a set-valued function. Once again, these definitions can be found for example in [AF90].

Definition V.3.6. A set-valued map F

$$
F: X \rightarrow 2^{Y}
$$

is said to be lower semicontinuous in $x$ if for any open set $V \in 2^{Y}$ such that $F(x) \cap V \neq \emptyset$ there exists a neighbourhood $U \subseteq X$ of $x$ such that $F\left(x^{\prime}\right) \cap V \neq \emptyset$ for any $x^{\prime} \in U$.

$F$ is said to be upper semicontinuous in $x$ if for any open neighbourhood $V$ of $F(x)$ there exists a neighbourhood $U$ of $x$ such that $F\left(x^{\prime}\right) \subseteq V$ for all $x^{\prime} \in U$.

Finally, $F$ is said to be continuous in $x$ if it is lower semicontinuous and upper semicontinuous in $x$.

A set-valued map is said to be continuous (lower semicontinuous, upper semicontinuous) if it is continuous (lower semicontinuous, upper semicontinuous) in $x$ for all $x \in X$.

As we mentioned before, in order to show that $F^{*}$ is lower semicontinuous we first want to show that there exist $(a(s), b(s)) \in M_{\text {ver }}^{\text {opt }}(s), s \geq 0$, in such a way that $a_{j}(s)$ is piecewise linear in $s$ for all $j=1 \ldots k$ as well as $b(s)$ is piecewise linear in $s$. We need some notation here.

Let us choose an arbitrary $\tilde{s} \leq \min \left\{\|a\|_{1}:(a, b) \in M_{\text {ver }}^{\text {opt }}\right\}$ and keep it fixed. Assume that $(a(\tilde{s}), b(\tilde{s})) \in M_{v e r}^{\text {opt }}(\tilde{s})$ is an optimal solution to $\left(P_{\text {ver }}(\tilde{s})\right)$. Note that the choice of $(a(\tilde{s}), b(\tilde{s}))$ need not to be unique.

We denote by $\mathcal{A}^{+}, \mathcal{A}^{-}$and $\mathcal{A}$ the sets of indices

$$
\begin{aligned}
& \mathcal{A}^{+}:=\mathcal{A}^{+}(a(\tilde{s}), b(\tilde{s}))=\left\{j: a_{j}(\tilde{s})>0\right\} \subseteq\{1, \ldots, k\}, \\
& \mathcal{A}^{-}:=\mathcal{A}^{-}(a(\tilde{s}), b(\tilde{s}))=\left\{j: a_{j}(\tilde{s})<0\right\} \subseteq\{1, \ldots, k\} \text { and }
\end{aligned}
$$




$$
\mathcal{A}:=\mathcal{A}(a(\tilde{s}), b(\tilde{s}))=\mathcal{A}^{+} \cup \mathcal{A}^{-}
$$

Recall that in Section $\mathrm{V} .2$ we denoted by $\mathcal{H}$ the set of hyperplanes in the dual space that correspond to one of the existing facilities and by $\mathcal{H}^{\prime \prime}$ the set of hyperplanes of the form $H_{j}=\left\{(a, b) \in \mathbb{R}^{k+1}: a_{j}=0\right\}$ for $j=1, \ldots, k$. Next we want to define a set $\mathcal{V}$ that contains the indices of all hyperplanes that pass through $(a(\tilde{s}), b(\tilde{s}))$ while either belonging to $\mathcal{H}$ or $\mathcal{H}^{\prime \prime}$. Remember that $\left(x_{i 1}, \ldots, x_{i k}, y_{i}\right)$ with $i=1, \ldots, n$ denote the existing facilities. In order to avoid double entries in the set $\mathcal{V}$, we rename the hyperplanes $H_{j} \in \mathcal{H}^{\prime \prime}$ by

$$
H_{n+j}:=H_{j}
$$

and we get

$$
\begin{aligned}
\mathcal{H}^{\prime \prime} & =\left\{H_{n+j}: j=1, \ldots, k\right\} \\
& =\left\{H_{i}: i=n+1, \ldots, n+k .\right\}
\end{aligned}
$$

Now we can denote by $\mathcal{V}_{\mathcal{H}}, \mathcal{V}_{\mathcal{H}^{\prime \prime}}$ and $\mathcal{V}$ the sets of indices

$$
\begin{aligned}
\mathcal{V}_{\mathcal{H}} & :=\mathcal{V}_{\mathcal{H}}(a(\tilde{s}), b(\tilde{s}))=\left\{i:(a(\tilde{s}), b(\tilde{s})) \in H_{i}\right\} \\
& =\left\{i: y_{i}-\left(\sum_{j \in \mathcal{A}} a_{j}(\tilde{s}) x_{i j}+b(\tilde{s})\right)=0\right\} \subseteq\{1, \ldots, n\}, \\
\mathcal{V}_{\mathcal{H}^{\prime \prime}} & :=\mathcal{V}_{\mathcal{H}^{\prime \prime}}(a(\tilde{s}), b(\tilde{s}))=\left\{i:(a(\tilde{s}), b(\tilde{s})) \in H_{i}\right\} \subseteq\{n+1, \ldots, n+k\} \text { and } \\
\mathcal{V} & :=\mathcal{V}_{\mathcal{H}} \cup \mathcal{V}_{\mathcal{H}^{\prime \prime}} \subseteq\{1, \ldots, n+k\} .
\end{aligned}
$$

We will refer to the set $\mathcal{A}$ as the active set of $(a(\tilde{s}), b(\tilde{s}))$ because it contains all indices of entries in $a(\tilde{s})$ that are "active" in the sense that they are non-zero.

The set $\mathcal{V}$ contains all indices of hyperplanes in the dual space that pass through $(a(\tilde{s}), b(\tilde{s}))$ not corresponding to a restriction on the $a$-vector. Therefore we will refer to $\mathcal{V}$ as the set of passing hyperplanes of $(a(\tilde{s}), b(\tilde{s}))$.

Furthermore we will refer to $\mathcal{V}_{\mathcal{H}}$ as the set of passed facilities of $(a(\tilde{s}), b(\tilde{s}))$ since this set contains all indices of existing facilities that correspond to a hyperplane in the dual space passing through $(a(\tilde{s}), b(\tilde{s}))$. In other words, the hyperplane in the initial space corresponding to the point $(a(\tilde{s}), b(\tilde{s}))$ passes through all existing 


\section{LOCATING HYPERPLANES WITH A RESTRICTION ON THE SLOPE USING VERTICAL DISTANCE

facilities $\left(x_{i}, y_{i}\right)$ with $i \in \mathcal{V}_{\mathcal{H}}$.

To $\mathcal{V}_{\mathcal{H}^{\prime \prime}}$ we will refer as the inactive set of $(a(\tilde{s}), b(\tilde{s}))$. The following remark will clarify why we do so.

Remark V.3.1. There exists a strong connection between $\mathcal{A}$ and $\mathcal{V}_{\mathcal{H}^{\prime \prime}}:$ It holds that $j \in \mathcal{A}$ if and only if $i=n+j \notin \mathcal{V}_{\mathcal{H}^{\prime \prime}}$. This is easy to see by just looking at the definitions of $\mathcal{A}$ and $\mathcal{V}_{\mathcal{H}^{\prime \prime}}$, respectively.

Due to Remark V.3.1 it is easy to see that if an entry $a_{j}(\tilde{s})$ of $a(\tilde{s})$ is "inactive" in the sense that $a_{j}(\tilde{s})=0$ then the corresponding index $n+j$ is an element of $\mathcal{V}_{\mathcal{H}^{\prime \prime}}$. Therefore it makes sense to refer to $\mathcal{V}_{\mathcal{H}^{\prime \prime}}$ as the inactive set of $(a(\tilde{s}), b(\tilde{s}))$.

Remark V.3.2. Note that there always exists an optimal solution $(a(s), b(s))$ of $\left(P_{\text {ver }}(s)\right), s \geq 0$, such that the set of passed facilities $\mathcal{V}_{\mathcal{H}}$ is non-empty. This is due to Theorem V.2.1.

We know from Theorem $\mathrm{V} .2 .3$ that $\|a(\tilde{s})\|_{1}=\tilde{s}$ holds. In addition we can assume that $(a(\tilde{s}), b(\tilde{s}))$ is a vertex of a cell $C$ defined by $\mathcal{H} \cup \mathcal{H}^{\prime}$ due to Theorem $\nabla .2 .1$. Since $\mathcal{H} \cup \mathcal{H}^{\prime} \cup \mathcal{H}^{\prime \prime}$ gives a subpartition of the cell partition induced by $\mathcal{H} \cup \mathcal{H}^{\prime}$, we can actually assume that $(a(\tilde{s}), b(\tilde{s}))$ is a vertex of a cell $\tilde{C} \in \tilde{\mathcal{C}}$ defined by $\mathcal{H} \cup \mathcal{H}^{\prime} \cup \mathcal{H}^{\prime \prime}$, the set of hyperplanes in the dual space. Without loss of generality, we furthermore assume that $\mathcal{V}$ is nonempty (see Remark $\overline{\mathrm{V} .3 .2}$ ) and that $\operatorname{sgn}(b(s))=1$.

Now we define two nonnegative real numbers $\underline{s}$ and $\bar{s}$ by

$$
\begin{aligned}
\underline{s}:= & \inf \left\{s^{\prime}: \text { for all } s \text { satisfying } s^{\prime} \leq s \leq \tilde{s} \text { there exists }(a(s), b(s)) \in M_{\text {ver }}^{\text {opt }}(s)\right. \\
& \text { s.t. } \left.\mathcal{A}^{+}(a(s), b(s))=\mathcal{A}^{+}, \mathcal{A}^{-}(a(s), b(s))=\mathcal{A}^{-} \text {and } \mathcal{V}(a(s), b(s))=\mathcal{V}\right\}
\end{aligned}
$$

and

$$
\begin{aligned}
\bar{s}:= & \sup \left\{s^{\prime}: \text { for all } s \text { satisfying } \tilde{s} \leq s \leq s^{\prime} \text { there exists }(a(s), b(s)) \in M_{\text {ver }}^{\text {opt }}(s)\right. \\
& \text { s.t. } \left.\mathcal{A}^{+}(a(s), b(s))=\mathcal{A}^{+}, \mathcal{A}^{-}(a(s), b(s))=\mathcal{A}^{-} \text {and } \mathcal{V}(a(s), b(s))=\mathcal{V}\right\}
\end{aligned}
$$

respectively.

Remark V.3.3. Remember that we have chosen $(a(\tilde{s}), b(\tilde{s})) \in M_{v e r}^{\text {opt }}(\tilde{s})$ for a fixed $\tilde{s}$. What we actually want to do in order to get a trajectory is to let s vary. 
Note that we defined $\underline{s}$ and $\bar{s}$ in the way that starting in $\tilde{s}$, the next time that there exists no optimal solution $(a(s), b(s))$ to $\left(P_{\text {ver }}(s)\right)$ with $\mathcal{A}^{+}(a(s), b(s))=\mathcal{A}^{+}$, $\mathcal{A}^{-}(a(s), b(s))=\mathcal{A}^{-}$and $\mathcal{V}(a(s), b(s))=\mathcal{V}$ will be in $\underline{s}$ if we decrease $\tilde{s}$ or in $\bar{s}$ if we increase $\tilde{s}$. This means that in $\underline{s}$ as well as in $\bar{s}$ some changes to $\mathcal{A}$ or $\mathcal{V}$ have to occur. This will play an important role later on.

Our aim is to show that there exist $(a(s), b(s)) \in M_{v e r}^{\text {opt }}(s), s \geq 0$, such that $a_{j}(s)$ is piecewise linear in $s$ for all $j=1 \ldots k$ and in addition $b(s)$ is piecewise linear in $s$. For the time being we want to restrict ourselves to the interval $(\underline{s}, \bar{s})$ and we show that there exist $(a(s), b(s)) \in M_{\text {ver }}^{\text {opt }}(s), s \geq 0$, such that $a_{j}(s)$ is linear in $s$ for all $j=1 \ldots k$ and in addition $b(s)$ is linear in $s$ on $(\underline{s}, \bar{s})$. Two cases can occur: Either $(\underline{s}, \bar{s})$ is not a real interval, that is $\underline{s}=\bar{s}=\tilde{s}$. Then we pick $(a(\tilde{s}), b(\tilde{s}))$ as an optimal solution to $\left(P_{\text {ver }}(\tilde{s})\right)$ and we are done.

The more interesting case is the one where $(\underline{s}, \bar{s})$ is a real interval with $\tilde{s} \in(\underline{s}, \bar{s})$.

We want to show that for $s \in(\underline{s}, \bar{s})$, we can choose certain $(a(s), b(s))$ from the set

$$
\left\{(a, b):(a, b) \in M_{v e r}^{\text {opt }}(s), \mathcal{A}^{+}(a, b)=\mathcal{A}^{+}, \mathcal{A}^{-}(a, b)=\mathcal{A}^{-} \text {and } \mathcal{V}(a, b)=\mathcal{V}\right\}
$$

(which is nonempty due to the definition of $\underline{s}$ and $\bar{s}$ ) in such a way that our chosen $(a(s), b(s))$ are linear in $s$. Let us denote these $(a(s), b(s))$ by

$$
\begin{gathered}
M_{\text {lin }}^{\text {opt }}(\underline{s}, \bar{s}):=\left\{(a(s), b(s)): s \in(\underline{s}, \bar{s}),(a(s), b(s)) \in M_{\text {ver }}^{\text {opt }}(s), \mathcal{A}^{+}(a(s), b(s))\right. \\
\left.=\mathcal{A}^{+}, \mathcal{A}^{-}(a(s), b(s))=\mathcal{A}^{-} \text {and } \mathcal{V}(a(s), b(s))=\mathcal{V}\right\}
\end{gathered}
$$

We look at the problem 


$$
\begin{aligned}
\min \sum_{i=1}^{n} d_{v e r}((a, b), & \left.H_{-x_{i}, y_{i}}\right) \\
\text { s.t. } \sum_{j \in \mathcal{A}^{+}} a_{j}-\sum_{j \in \mathcal{A}^{-}} a_{j} & =s \\
\sum_{j \in \mathcal{A}} a_{j} x_{i j}+b & =y_{i} \forall i \in \mathcal{V}_{\mathcal{H}} \\
a_{j} & \geq 0 \forall j \in \mathcal{A}^{+} \\
a_{j} & \leq 0 \forall j \in \mathcal{A}^{-} \\
a_{j} & =0 \forall j \text { with } n+j \in \mathcal{V}_{\mathcal{H}^{\prime \prime}} \\
b & \geq 0 .
\end{aligned}
$$

By the way we defined $(\tilde{P}(s))$ it is obvious that $(a(\tilde{s}), b(\tilde{s}))$ is an optimal solution for $(\tilde{P}(\tilde{s}))$. We want to reformulate $(\tilde{P}(s))$ in order to clarify that it is a linear program. Afterwards we will see that $(a(\tilde{s}), b(\tilde{s}))$ is indeed a basic solution to $(\tilde{P}(\tilde{s}))$ which will help us to show the linearity of the $(a(s), b(s)) \in M_{\text {lin }}^{\text {opt }}(\underline{s}, \bar{s})$. We start with the following lemma.

Lemma V.3.1. It holds that the sets $R(a(s), b(s))$ and $L(a(s), b(s))$ as defined in Section V.1 are identical for all $(a(s), b(s)) \in M_{\text {lin }}^{\text {opt }}(\underline{s}, \bar{s})$, meaning that

$$
R(a(s), b(s))=R(a(\tilde{s}), b(\tilde{s}))
$$

and

$$
L(a(s), b(s))=L(a(\tilde{s}), b(\tilde{s}))
$$

for $\operatorname{all}(a(s), b(s)) \in M_{\text {lin }}^{\text {opt }}(\underline{s}, \bar{s})$.

Proof. It is true by definition of $\underline{s}$ and $\bar{s}$ that all $(a(s), b(s)) \in M_{\text {lin }}^{\text {opt }}(\underline{s}, \bar{s})$ have the same set of passing hyperplanes $\mathcal{V}$. In particular, they have the same set of passed facilities $\mathcal{V}_{\mathcal{H}}$.

Assume that there exists an index $i$ such that $i \in L\left(a\left(s_{1}\right), b\left(s_{1}\right)\right)$ while $i \notin$ $L\left(a\left(s_{2}\right), b\left(s_{2}\right)\right)$ with $\left(a\left(s_{1}\right), b\left(s_{1}\right)\right),\left(a\left(s_{2}\right), b\left(s_{2}\right)\right) \in M_{\text {lin }}^{\text {opt }}(\underline{s}, \bar{s})$ and $s_{1}<s_{2}$. Since the set of passed facilities is $\mathcal{V}_{\mathcal{H}}$ for both $\left(a\left(s_{1}\right), b\left(s_{1}\right)\right)$ and $\left(a\left(s_{2}\right), b\left(s_{2}\right)\right)$, it has 
to hold that $i \in R\left(a\left(s_{2}\right), b\left(s_{2}\right)\right)$. But this can only happen if the hyperplane corresponding to $i$ intersects the hyperplanes corresponding to the indices in $\mathcal{V}_{\mathcal{H}}$ in $\left(a_{\text {intersect }}, b_{\text {intersect }}\right) \in \mathbb{R}^{k+1}$ such that $\left\|a_{\text {intersect }}\right\|_{1}=s^{\prime}$ with $s^{\prime} \in\left(s_{1}, s_{2}\right)$. This would mean that $i \in \mathcal{V}_{\mathcal{H}}\left(a\left(s^{\prime}\right), b\left(s^{\prime}\right)\right)$ for $\left(a\left(s^{\prime}\right), b\left(s^{\prime}\right)\right) \in M_{\text {lin }}^{\text {opt }}(\underline{s}, \bar{s})$ and therefore $\mathcal{V}_{\mathcal{H}}\left(a\left(s^{\prime}\right), b\left(s^{\prime}\right)\right) \neq \mathcal{V}_{\mathcal{H}}$, a contradiction.

We can rewrite the objective function $f_{v e r}$ by

$$
\begin{aligned}
f_{v e r}(a, b)= & \sum_{i=1}^{n} d_{v e r}\left((a, b), H_{-x_{i}, y_{i}}\right) \\
= & \sum_{i \in R(a, b)} y_{i}-\left(a_{1} x_{i 1}+\ldots+a_{k} x_{i k}+b\right) \\
& +\sum_{i \in L(a, b)} a_{1} x_{i 1}+\ldots+a_{k} x_{i k}+b-y_{i} \\
= & \sum_{i \in R(a, b)} y_{i}-\sum_{i \in L(a, b)} y_{i} \\
& +a_{1}\left(\sum_{i \in L(a, b)} x_{i 1}-\sum_{i \in R(a, b)} x_{i 1}\right) \\
& +a_{2}\left(\sum_{i \in L(a, b)} x_{i 2}-\sum_{i \in R(a, b)} x_{i 2}\right) \\
& \left.+\ldots \sum_{i \in L(a, b)} x_{i k}-\sum_{i \in R(a, b)} x_{i k}\right) \\
& +a_{k}\left(\sum_{i \in R(a, b)} b .\right.
\end{aligned}
$$

For the time being, we are only interested in $s \in(\underline{s}, \bar{s})$ which means that the term $\sum_{i \in R(a, b)} y_{i}-\sum_{i \in L(a, b)} y_{i}$ is a constant. To be more precise, the sets $R(a(s), b(s))$ and $L(a(s), b(s))$ are constant for all $(a(s), b(s)) \in M_{\text {lin }}^{\text {opt }}(\underline{s}, \bar{s})$ due to Lemma V.3.1. Therefore the above mentioned sum is not important for minimization and can be left out. In the following, we will denote the constant sets $R(a(s), b(s))$ and $L(a(s), b(s))$ by $R$ and $L$, respectively. 
Let $\mathcal{A}=\left\{j_{1}, \ldots, j_{m}\right\} \subseteq\{1, \ldots, k\}$ and assume without loss of generality $\mathcal{A}^{+}=$ $\left\{j_{1}, j_{2}, \ldots, j_{l}\right\}$ while $\mathcal{A}^{-}=\left\{j_{l+1}, j_{l+2}, \ldots, j_{m}\right\}$ with $l \leq m$. Define

$$
c_{\nu}:=\left\{\begin{array}{l}
\sum_{i \in L} x_{i j}-\sum_{i \in R} x_{i j} \text { for } \nu=1 \ldots m \\
|L|-|R| \text { for } \nu=m+1 .
\end{array}\right.
$$

Then it is $c=\left(c_{1}, \ldots, c_{m+1}\right)^{t} \in \mathbb{R}^{m+1}$. Our variables we denote by $a^{\prime}$ where

$$
a^{\prime}:=\left(a_{j_{1}}, \ldots, a_{j_{m}}, b\right)^{t} \in \mathbb{R}^{m+1}
$$

Now the dimensions of $c$ and $a^{\prime}$ fit together in the sense that we can look at $c^{t} a^{\prime}$. It holds that

$$
f_{v e r}(a, b)=c^{t} a^{\prime}
$$

for all $(a, b)$ with active set $\mathcal{A}$ and set of passing hyperplanes $\mathcal{V}$.

Note that such an active set $\mathcal{A}=\left\{j_{1}, \ldots, j_{m}\right\}$ directly implies an inactive set $\mathcal{V}_{\mathcal{H}^{\prime \prime}}=\{n+j: j \notin \mathcal{A}\}$ due to Remark V.3.1.

If we now assume $\mathcal{V}_{\mathcal{H}}=\left\{i_{1}, \ldots, i_{q}\right\} \subseteq\{1, \ldots, n\}$ and define a matrix $A \in$ $\mathbb{R}^{(q+1) \times(m+1)}$ by

$$
A:=\left(\begin{array}{ccccccc}
1 & \ldots & 1 & -1 & \ldots & -1 & 0 \\
x_{i_{1} j_{1}} & \ldots & x_{i_{1} j_{l}} & x_{i_{1} j_{l+1}} & \ldots & x_{i_{1} j_{m}} & 1 \\
\vdots & \ddots & \vdots & \vdots & \ddots & \vdots & \vdots \\
x_{i_{q} j_{1}} & \ldots & x_{i_{q} j_{l}} & x_{i_{q} j_{l+1}} & \ldots & x_{i_{q} j_{m}} & 1
\end{array}\right)
$$

and a vector $\tilde{y}(s) \in \mathbb{R}^{q+1}$ by

$$
\tilde{y}(s):=\left(s, y_{i_{1}}, \ldots, y_{i_{q}}\right)^{t}
$$

we can rewrite $(\tilde{P}(s))$ for $s \in(\underline{s}, \bar{s})$ as 
$\min c^{t} a^{\prime}$

$$
\text { s.t. } \begin{aligned}
A a^{\prime} & =\tilde{y}(s) \\
& a_{\mu}^{\prime} \geq 0 \text { for } \mu=1 \ldots l \\
a_{\mu}^{\prime} & \leq 0 \text { for } \mu=l+1 \ldots m \\
a_{\mu}^{\prime} & \geq 0 \text { for } \mu=m+1 .
\end{aligned}
$$

We assume without loss of generality that $m \geq q$ and that the rank of $A$ is $q+1$. Otherwise the system of linear equations $A a^{\prime}=\tilde{y}(\tilde{s})$ has exactly one or none solution or there exist linearly dependent rows of $A$ which can be left out. In the first case, $(\tilde{P}(\tilde{s}))$ is not interesting as an optimization problem. The latter case corresponds to a degenerate case where there intersect more than $q+1$ hyperplanes. With the reformulation at hand, it is obvious that $(\tilde{P}(s))$ is a linear program for $s \in(\underline{s}, \bar{s})$. Now we want to prove the following lemma.

Lemma V.3.2. Let $\tilde{s} \geq 0$ such that $(\underline{s}, \bar{s})$ is a real interval with $\tilde{s} \in(\underline{s}, \bar{s})$ and let $(a(\tilde{s}), b(\tilde{s})) \in M_{\text {ver }}^{\text {opt }}(\tilde{s})$ be an optimal solution for $\left(P_{\text {ver }}(\tilde{s})\right)$ with active set $\mathcal{A}=$ $\mathcal{A}^{+} \cup \mathcal{A}^{-}$where $\mathcal{A}^{+}=\left\{j_{1}, \ldots, j_{l}\right\}$ and $\mathcal{A}^{-}=\left\{j_{l+1}, \ldots, j_{m}\right\}$ and set of passed hyperplanes $\mathcal{V}_{\mathcal{H}}=\left\{i_{1}, \ldots, i_{q}\right\}$. Furthermore let $(\tilde{P}(s))$ be the linear program

$$
\begin{aligned}
\min & c^{t} a^{\prime} \\
\text { s.t. } A a^{\prime} & =\tilde{y}(s) \\
a_{\mu}^{\prime} & \geq 0 \text { for } \mu=1 \ldots l \\
a_{\mu}^{\prime} & \leq 0 \text { for } \mu=l+1 \ldots m \\
a_{\mu}^{\prime} & \geq 0 \text { for } \mu=m+1
\end{aligned}
$$

as formulated above.

Then $a^{\prime}(\tilde{s}):=\left(a(\tilde{s})_{j_{1}}, \ldots, a(\tilde{s})_{j_{m}}, b(\tilde{s})\right)^{t}$ is an optimal basic solution for $(\tilde{P}(\tilde{s}))$.

Proof. We already know that $a^{\prime}(\tilde{s})$ is an optimal solution to $\left(P_{v e r}(\tilde{s})\right)$ by choice of $(a(\tilde{s}), b(\tilde{s}))$ and definition of $a^{\prime}(\tilde{s})$.

The way we constructed the alternative program $(\tilde{P}(\tilde{s}))$ makes sure that $a^{\prime}(\tilde{s}):=$ $\left(a(\tilde{s})_{j_{1}}, \ldots, a(\tilde{s})_{j_{m}}, b(\tilde{s})\right)^{t}$ is a vertex of the polyhedron defined by the constraints of 


\section{LOCATING HYPERPLANES WITH A RESTRICTION ON THE SLOPE USING VERTICAL DISTANCE

$(\tilde{P}(\tilde{s}))$. But the vertices of this polyhedron correspond to feasible basic solutions for $(\tilde{P}(\tilde{s}))$ which is a well-known fact in linear optimization theory. This shows that $a^{\prime}(\tilde{s})$ is indeed an optimal basic solution to $(\tilde{P}(\tilde{s}))$.

Actually, choosing $(a(\tilde{s}), b(\tilde{s}))$ and constructing $(\tilde{P}(\tilde{s}))$ based on $\mathcal{A}$ and $\mathcal{V}$ already makes sure that a basis $B$ of $A$ such that $A_{B}=A$ leads to an optimal solution for $(\tilde{P}(\tilde{s}))$. This is a nice fact which leads to a result that will help us when we want to construct an optimal trajectory for $\left(P_{v e r}(s)\right)$ explicitly.

Lemma V.3.3. Let $\tilde{s} \geq 0$ such that $(\underline{s}, \bar{s})$ is a real interval with $\tilde{s} \in(\underline{s}, \bar{s})$ and let $(a(\tilde{s}), b(\tilde{s})) \in M_{v e r}^{\text {opt }}(\tilde{s})$ and $a^{\prime}(\tilde{s})$ be defined as in the previous lemma, let $\mathcal{A}=$ $\left\{j_{1}, \ldots, j_{m}\right\}$ and $\mathcal{V}_{\mathcal{H}}=\left\{i_{1}, \ldots, i_{q}\right\}$. If $B$ is a basis of $A$ such that $a^{\prime}(\tilde{s})=A_{B}^{-1} \tilde{y}(\tilde{s})$ it holds that $A_{B}=A$ and $m=q$. In particular, $B$ is the only basis of $A$.

Proof. From Lemma V.3.2, we know that $a^{\prime}(\tilde{s})$ is a basic solution to the linear problem $(\tilde{P}(\tilde{s}))$. This means that there exists a basis $B$ of $A$ such that

$$
a^{\prime}(\tilde{s})=A_{B}^{-1} \tilde{y}(\tilde{s})
$$

Remember that we assume $\operatorname{rank}(A)=q+1$ and $m \geq q$. Since $A$ has $\operatorname{rank}(A)=$ $q+1$, it must hold that $A_{B} \in \mathbb{R}^{(q+1) \times(q+1)}$ and therefore $a^{\prime}(\tilde{s})=A_{B}^{-1} \tilde{y}(\tilde{s}) \in \mathbb{R}^{q+1}$. On the other hand, we know that by choice of $a^{\prime}(\tilde{s})$ it holds that $a^{\prime}(\tilde{s}) \in \mathbb{R}^{m+1}$ with $a^{\prime}(\tilde{s})_{\mu} \neq 0$ for $\mu=1, \ldots, m+1$ and therefore $a^{\prime}(\tilde{s})_{B}=a^{\prime}(\tilde{s}) \in \mathbb{R}^{m+1}$. But that means that $q=m$ and therefore $A_{B}=A$. Since $q=m$ there cannot exist another different basis and therefore $B$ is the unique basis of $A$.

Lemma V.3.2 together with Lemma V.3.3 tell us that $(a(\tilde{s}), b(\tilde{s}))$ can be written as

$$
a^{\prime}(\tilde{s})=A^{-1} \tilde{y}(\tilde{s})
$$

as an optimal basic solution $a^{\prime}(\tilde{s})$ of $(\tilde{P}(\tilde{s}))$. To be more precise, it holds that

$$
a^{\prime}(\tilde{s})_{\mu}=\tilde{x}_{\mu 1} \cdot \tilde{s}+\sum_{\nu=1}^{m} \tilde{x}_{\mu(\nu+1)} \tilde{y}(\tilde{s})_{\nu}\left\{\begin{array}{l}
>0 \text { for } \mu=1 \ldots l \text { and } \mu=m+1 \\
<0 \text { for } \mu=l+1 \ldots m .
\end{array}\right.
$$


where $\tilde{x}_{\mu \nu}$ are the entries of $A^{-1}$. Note that these entries only depend on the given entries of the existing $x_{i} \in \mathbb{R}^{k}$.

This observation leads to a theorem that is crucial for the proof of the existence of $(a(s), b(s)) \in M_{v e r}^{o p t}(s), s \in(\underline{s}, \bar{s})$, such that $a_{j}(s)$ is linear in $s$ for all $j=1 \ldots k$ and in addition $b(s)$ is linear in $s$.

Theorem V.3.2. Let $A \in \mathbb{R}^{(q+1) \times(m+1)}$ be the matrix in $(\tilde{P}(s))$. It holds that $m=q$ and for any $s \in(\underline{s}, \bar{s})$ the only basis of $A$ is $B$ such that $A_{B}=A$.

Proof. For any $s \in(\underline{s}, \bar{s})$, the matrix $A$ in $(\tilde{P}(s))$ is indeed the same as in $(\tilde{P}(\tilde{s}))$ since the entries of $A$ only depend on the given entries of the existing $x_{i} \in \mathbb{R}^{k}$ as we already mentioned above. Therefore $B$ with $A_{B}=A$ is a basis of $A$ for any $s \in(\underline{s}, \bar{s})$ due to Lemma V.3.3. This lemma also tells us that $q=m$, therefore $B$ is the only basis of $A$.

If $s \in(\underline{s}, \bar{s})$ with $s \neq \tilde{s}$, the only thing that changes in $(\tilde{P}(s))$ is the $\tilde{y}$-vector, namely $\tilde{y}(s)_{1}=s$ instead of $\tilde{y}(\tilde{s})_{1}=\tilde{s}$ as it was the case in $(\tilde{P}(\tilde{s}))$. However, it is true that $\tilde{y}(s)_{\nu}=\tilde{y}(\tilde{s})_{\nu}$ for any $\nu \neq 1$.

Now that we know that $B$ with $A_{B}=A$ is a basis of $A$ for any $s \in(\underline{s}, \bar{s})$ due to Theorem V.3.2, let us look at $A^{-1} \tilde{y}(s)$.

$$
\begin{aligned}
A^{-1} \tilde{y}(s)_{\mu} & =\tilde{x}_{\mu 1} \cdot s+\sum_{\nu=1}^{m} \tilde{x}_{\mu(\nu+1)} \tilde{y}(\tilde{s})_{\nu} \\
& =(s-\tilde{s}) \tilde{x}_{\mu 1}+\tilde{x}_{\mu 1} \cdot \tilde{s}+\sum_{\nu=1}^{m} \tilde{x}_{\mu(\nu+1)} \tilde{y}(\tilde{s})_{\nu} \\
& =(s-\tilde{s}) \tilde{x}_{\mu 1}+a^{\prime}(\tilde{s})_{\mu} .
\end{aligned}
$$

This defines a feasible solution to $(\tilde{P}(s))$ for any $s \in(\underline{s}, \bar{s})$ since $(\tilde{P}(s))$ is a linear program for $s \in(\underline{s}, \bar{s})$ and as such it has an optimal basic solution. Since $B$ with $A_{B}=A$ is the only basis of $A$ due to Theorem $\overline{V .3 .2}, A^{-1} \tilde{y}(s)$ is an optimal solution to $(\tilde{P}(s))$ and therefore in particular feasible. But this means that

$$
A^{-1} \tilde{y}(s)_{\mu}\left\{\begin{array}{l}
>0 \text { for } \mu=1 \ldots l \text { and } \mu=m+1 \\
<0 \text { for } \mu=l+1 \ldots m
\end{array}\right.
$$


If we define $a^{\prime}(s)$ by

$$
a^{\prime}(s):=A^{-1} \tilde{y}(s)
$$

for all $s \in(\underline{s}, \bar{s})$ we get that $a^{\prime}(s)$ is an optimal basic solution to $(\tilde{P}(s))$ for any $s \in(\underline{s}, \bar{s})$. Alternatively, we can write $a^{\prime}(s)$ as

$$
a_{\mu}^{\prime}(s)=(s-\tilde{s}) \tilde{x}_{\mu 1}+a^{\prime}(\tilde{s})_{\mu}, \mu=1, \ldots, m+1 .
$$

Lemma V.3.4. Let $s \in(\underline{s}, \bar{s})$ and let $a^{\prime}(s)=A^{-1} \tilde{y}(s)$. Then $a^{\prime}(s)$ is an optimal basic solution for $(\tilde{P}(s))$.

Proof. This lemma is a direct consequence of the definition of $a^{\prime}(s)$ and Theorem V.3.2.

Finally, we know that there exist optimal solutions $a^{\prime}(s)$ for $(\tilde{P}(s)), s \in(\underline{s}, \bar{s})$, in such a way that $a^{\prime}(s)_{\mu}$ is linear in $s$ for $\mu=1, \ldots, m+1$.

Theorem V.3.3. Let $s \in(\underline{s}, \bar{s})$ and let $a^{\prime}(s)=A^{-1} \tilde{y}(s)$ be defined as above. Then $a^{\prime}(s)$ is an optimal solution for $(\tilde{P}(s))$ and $a^{\prime}(s)$ is linear in $s$ for $\mu=1, \ldots, m+1$.

Proof. Fortunately, there is nothing left to prove. Due to Theorem V.3.2 and Lemma V.3.4 we know that $a^{\prime}(s)$ is the unique optimal basic solution to $(\tilde{P}(s))$ for any $s \in(\underline{s}, \bar{s})$, and as such we can write all $a^{\prime}(s)$ as

$$
a_{\mu}^{\prime}(s)=(s-\tilde{s}) \tilde{x}_{\mu 1}+a^{\prime}(\tilde{s})_{\mu}, \mu=1, \ldots, m+1,
$$

$a^{\prime}(s)_{\mu}$ clearly is linear in $s$ for $\mu=1, \ldots, m+1$

Up to now, we showed that there exist solutions to our problem $(\tilde{P}(s))$ constructed from an optimal solution for $\left(P_{\text {ver }}(\tilde{s})\right)$ which are linear in $s$ in each component. Remember that our actual aim is to show that there exist optimal solutions $(a(s), b(s))$ of $\left(P_{\text {ver }}(s)\right), s \geq 0$, such that $a(s)_{j}$ is piecewise linear in $s$ for $j=1, \ldots, k$ as well as $b(s)$ is piecewise linear in $s$.

Remember that $\mathcal{A}=\left\{j_{1}, \ldots, j_{m}\right\}$. Note that $a^{\prime}(s)$ corresponds to a feasible solution $(a(s), b(s))$ for $\left(P_{v e r}(s)\right)$ for all $s \in(\underline{s}, \bar{s})$ with 


$$
\begin{aligned}
a(s)_{j_{\mu}} & =a^{\prime}(s)_{\mu} \text { for } j_{\mu} \in \mathcal{A} \\
a(s)_{j} & =0 \text { for } j \notin \mathcal{A} \text { and } \\
b(s) & =a^{\prime}(s)_{m+1} .
\end{aligned}
$$

It is clear by definition of $(a(s), b(s))$ that it has active set $\mathcal{A}$ and set of passing hyperplanes $\mathcal{V}$ for all $s \in(\underline{s}, \bar{s})$. What we want to show is that $(a(s), b(s))$ defined as above is an element of $M_{\text {lin }}^{\text {opt }}(\underline{s}, \bar{s})$ for all $s \in(\underline{s}, \bar{s})$, that is to say $(a(s), b(s))$ in addition to the aforementioned properties is an optimal solution to $\left(P_{v e r}(s)\right)$ for all $s \in(\underline{s}, \bar{s})$.

Theorem V.3.4. Let $s \in(\underline{s}, \bar{s})$ and let $(a(s), b(s))$ be defined as above. Then $(a(s), b(s)) \in M_{\text {lin }}^{\text {opt }}(\underline{s}, \bar{s})$.

Proof. Let $s \in(\underline{s}, \bar{s})$ and assume that $(a(s), b(s)) \notin M_{l i n}^{o p t}(\underline{s}, \bar{s})$. By the choice of the interval $(\underline{s}, \bar{s})$ there exists an optimal solution $(\hat{a}(s), \hat{b}(s))$ to $\left(P_{\text {ver }}(s)\right)$ such that $(\hat{a}(s), \hat{b}(s)) \in M_{\text {lin }}^{\text {opt }}(\underline{s}, \bar{s})$. This means $(\hat{a}(s), \hat{b}(s))$ has active set $\mathcal{A}$ and set of passing hyperplanes $\mathcal{V}$. Additionally, it holds that

$$
f_{v e r}(\hat{a}(s), \hat{b}(s))<f_{v e r}(a(s), b(s)) .
$$

If we again define $\hat{a}^{\prime}(s)$ by just considering the entries of $\hat{a}(s)$ that are nonzero and $\hat{b}$ as we did before $\hat{a}^{\prime}(s)$ is a feasible solution to $(\tilde{P}(s))$. By the way we constructed $(a(s), b(s))$ we know that the corresponding $a^{\prime}(s)$ is an optimal solution to $(\tilde{P}(s))$, which means that

$$
c^{t} a^{\prime}(s) \leq c^{t} \hat{a}^{\prime}(s)
$$

But we know that

$$
c^{t} a^{\prime}=f_{v e r}(a, b)
$$

for all $(a, b)$ with active set $\mathcal{A}$ and set of passing hyperplanes $\mathcal{V}$ by construction of $c$ and $a^{\prime}$. Since both $(\hat{a}(s), \hat{b}(s))$ and $(a(s), b(s))$ fulfil this condition it follows that 


$$
f_{v e r}(a(s), b(s)) \leq f_{v e r}(\hat{a}(s), \hat{b}(s))
$$

a contradiction.

Therefore it holds that $(a(s), b(s)) \in M_{\text {lin }}^{\text {opt }}(\underline{s}, \bar{s})$.

Putting our results together we get the following corollary.

Corollary V.3.1. There exist $(a(s), b(s)) \in M_{\text {ver }}^{\text {opt }}(s), s \in(\underline{s}, \bar{s})$, in such a way that $a(s)_{j}$ is linear in $s$ for all $j=1 \ldots k$ as well as $b(s)$ is linear in $s$.

Proof. Let $s \in(\underline{s}, \bar{s})$. If we choose $(a(s), b(s))$ as in Theorem V.3.4, we know that $(a(s), b(s))$ is an optimal solution to $\left(P_{v e r}(s)\right)$. Due to the choice of $(a(s), b(s))$ and Theorem V.3.3, $a(s)_{j}$ is linear in $s$ for all $j=1 \ldots k$ and $b(s)$ is linear in $s$.

We already mentioned in Remark V.3.3 that in $\underline{s}$ as well as in $\bar{s}$ some changes have to occur concerning the active set $\mathcal{A}$ or the set of passing hyperplanes $\mathcal{V}$. Now we want to specify how these changes look like.

In order to do so, note that the linearity of $(a(s), b(s))$ on the interval $(\underline{s}, \bar{s})$ has a geometrical interpretation. So far, we denoted by $\mathcal{C}$ the set of cells defined through the hyperplanes $H \in \mathcal{H}$ in the dual space corresponding to existing facilities. If we consider the hyperplanes $H \in \mathcal{H}^{\prime}$ in the dual space that correspond to a restriction on the $a$-vector and all hyperplanes $H_{j} \in \mathcal{H}^{\prime \prime}, j=1, \ldots, k$, in addition, we get the set of cells $\tilde{\mathcal{C}}$. Let us now introduce a third set of cells we want to denote by $\mathcal{C}^{\prime}$, namely the set of cells induced by the partition of the dual $\mathbb{R}^{k+1}$ given by all hyperplanes corresponding to existing facilities and all hyperplanes $H_{j}, j=1, \ldots, k$. Sticking to our notation, $\mathcal{C}^{\prime}$ is the set of cells induced by the hyperplanes in $\mathcal{H} \cup \mathcal{H}^{\prime \prime}$.

Lemma V.3.5. Let $(\underline{s}, \bar{s})$ be a real interval with $\tilde{s} \in(\underline{s}, \bar{s})$. If we choose $(a(s), b(s)) \in M_{v e r}^{\text {opt }}(s)$ for $s \in(\underline{s}, \bar{s})$ as we described before, the obtained optimal trajectory limited to the interval $(\underline{s}, \bar{s})$ follows an edge of a cell $C^{\prime} \in \mathcal{C}^{\prime}$ defined by the sets of hyperplanes $\mathcal{H}$ and $\mathcal{H}^{\prime \prime}$.

Proof. Remember that all $(a(s), b(s))$ lying on our optimal trajectory for $s \in(\underline{s}, \bar{s})$ share the constant active set $\mathcal{A}=\left\{j_{1}, \ldots, j_{m}\right\}$ as well as the constant set of passing 


\section{V.3. THE EXISTENCE OF A CONTINUOUS OPTIMAL}

hyperplanes $\mathcal{V}$, which means that they share the constant set of passed facilities $\mathcal{V}_{\mathcal{H}}=\left\{i_{1}, \ldots, i_{q}\right\}$ in particular.

The set of passed facilities $\mathcal{V}_{\mathcal{H}}$ tells us that our $(a(s), b(s))$ lie in the intersection of the $q$ hyperplanes $H_{-x_{i_{1}}, y_{i_{1}}}, \ldots, H_{-x_{i_{q}}, y_{i_{q}}}$. The active set $\mathcal{A}$ together with Remark V.3.1 tells us that our $(a(s), b(s))$ lie in the intersection of the $k-m$ hyperplanes $H_{j}$ with $j \notin \mathcal{A}$ as well. We know from Theorem V.3.2 that it is $m=q$. Altogether we get that our $(a(s), b(s))$ lie in the intersection of $k$ hyperplanes from the set $\mathcal{H} \cup \mathcal{H}^{\prime \prime}$ and since we are in the $\mathbb{R}^{k+1}$, these $k$ hyperplanes define an edge of a cell $C^{\prime} \in \mathcal{C}^{\prime}$.

As long as we can find a feasible solution $(a(s), b(s))$ to $\left(P_{\text {ver }}(s)\right)$ lying on the edge of $C^{\prime}$ defined by $\mathcal{A}$ and $\mathcal{V}$ this solution will be optimal as well since it corresponds to an optimal basic solution $a^{\prime}(s)$ of $(\tilde{P}(s))$. Anyhow, if we let $s$ decrease or increase we will eventually reach a vertex of $C^{\prime}$ and our trajectory can no longer follow the same edge.

Reaching a vertex of $C^{\prime}$ at some $\hat{s}$ means that another hyperplane $H \in \mathcal{H} \cup \mathcal{H}^{\prime \prime}$ intersects with the $k$ hyperplanes which define the edge of $C^{\prime}$. In other words, another index $i \notin \mathcal{V}$ joins the set of passing hyperplanes to define $\mathcal{V}(\hat{s})=\mathcal{V} \cup$ $\{i\}$. Note that if the index $i \notin \mathcal{V}$ that joins the new set of passing hyperplanes corresponds to a hyperplane $H_{-x_{i}, y_{i}} \in \mathcal{H}$, that is $i \in\{1, \ldots, n\}$, this means that the index $i$ corresponding to the existing facility $\left(x_{i}, y_{i}\right)$ joins the set of passed facilities $\mathcal{V}_{\mathcal{H}}(\hat{s})=\mathcal{V}_{\mathcal{H}} \cup\{i\}$. If on the other hand the index $i \notin \mathcal{V}$ that joins the new set of passing hyperplanes corresponds to a hyperplane $H_{i} \in \mathcal{H}^{\prime \prime}$, that is $i \in\{n+1, \ldots, n+k\}$, this means that the index $i$ joins the inactive set $\mathcal{V}_{\mathcal{H}^{\prime \prime}}(\hat{s})=$ $\mathcal{V}_{\mathcal{H}^{\prime \prime}} \cup\{i\}$. Due to Remark V.3.1 this is equivalent to the corresponding $j \in \mathcal{A}$ leaving the active set and therefore $a_{j}$ becoming zero, that means $\mathcal{A}(\hat{s})=\mathcal{A} \backslash\{j\}$.

Remark V.3.4. In [EHJT04] and in [WGZ06], the action of $a(s)_{j}$ with $j \in \mathcal{A}$ becoming zero with changing $s$ as well as the action of an index $i \notin \mathcal{V}_{\mathcal{H}}$ joining $\mathcal{V}$ with changing $s$ is called an event.

We want to stick to this notation: Whenever a new index $i$ corresponding to a hyperplane $H \in \mathcal{H} \cup \mathcal{H}^{\prime \prime}$ joins the set of passing hyperplanes $\mathcal{V}$ at some $s$ we will say that an event happens in $s$ in the following. Depending on whether the joining index $i$ corresponds to a hyperplane in $\mathcal{H}$ or in $\mathcal{H}^{\prime \prime}$, an event is equivalent 


\section{LOCATING HYPERPLANES WITH A RESTRICTION ON THE SLOPE USING VERTICAL DISTANCE

to an index $i \notin \mathcal{V}$ corresponding to an existing facility joining the set of passing hyperplanes $\mathcal{V}$, or the set of passed facilities $\mathcal{V}_{\mathcal{H}}$ to be more precise, or to $a(s)_{j}$ becoming zero for some $j \in \mathcal{A}$ to an index $i \notin \mathcal{V}$ corresponding to an existing facility joining the set of passing hyperplanes $\mathcal{V}$, or the set of passed facilities $\mathcal{V}_{\mathcal{H}}$ to be more precise.

In general, it can of course happen that more than one event takes place at a time. Therefore, like [EHJT04] do for their LASSO regression method, we assume a one at a time condition, which means that each change of $\mathcal{V}$ only involves a single index $i$. This is equivalent to the condition that the changes of $\mathcal{A}$ never involve more than a single index $j$, the ones of $\mathcal{V}_{\mathcal{H}}$ never involve more than a single index $i$ and in addition either $\mathcal{A}$ changes or $\mathcal{V}_{\mathcal{H}}$ changes, never both at the same time. This can always be realized by changing the $y$ values of the existing facilities the slightest bit which should not have too much impact on the value of our objective function.

Assuming this condition, only one event happens at a time. Anything we will present here would work in a "many at a time" situation as well, here the one at a time condition is just a technical detail for simplicity of notation. It is more interesting when it comes to the construction of an optimal trajectory.

As we already pointed out, our $(a(s), b(s))$ follow an edge of a cell $C^{\prime} \in \mathcal{C}^{\prime}$ defined by $\mathcal{A}$ and $\mathcal{V}$ until we reach a vertex of $C^{\prime}$. Now that we introduced the notion of an event in Remark V.3.4, it is easy to see that this is equivalent to saying that $\mathcal{A}$ and $\mathcal{V}$ of our $(a(s), b(s))$ are constant until an event happens. Note that $\underline{s}$ and $\bar{s}$ are defined in the way that starting in $\tilde{s}$, the next event will take place in $\underline{s}$ if we decrease $s$ and in $\bar{s}$ if we increase $s$. In other words, $(a(\underline{s}), b(\underline{s}))$ and $(a(\bar{s}), b(\bar{s}))$ are different vertices of a cell $C^{\prime} \in \mathcal{C}^{\prime}$.

Remember that we distinguished between the two cases of $(\underline{s}, \bar{s})$ being just a single point, namely $\tilde{s}$, and $(\underline{s}, \bar{s})$ being a real interval with $\tilde{s} \in(\underline{s}, \bar{s})$. As a matter of fact, the first case is equivalent to assuming that an event has happened in $\tilde{s}$ (or to assuming that our starting solution $(a(\tilde{s}), b(\tilde{s}))$ is a vertex of a cell in $\left.\mathcal{C}^{\prime}\right)$ while the latter case is equivalent to assuming that no event has happened in $\tilde{s}$ (or to assuming that our starting solution $(a(\tilde{s}), b(\tilde{s}))$ is no vertex of a cell in $\left.\mathcal{C}^{\prime}\right)$.

The results of this section up to now enable us to show that there exists an optimal 
trajectory to $\left(P_{\text {ver }}(s)\right)$ which is piecewise linear in each component.

Theorem V.3.5. There exist $(a(s), b(s)) \in \mathbb{R}^{k+1}$ such that $(a(s), b(s)) \in M_{\text {ver }}^{\text {opt }}(s)$, $s \geq 0$, and $a(s)_{j}$ as well as $b(s)$ are piecewise linear in $s$. In other words, there exists an optimal trajectory for $\left(P_{\text {ver }}(s)\right)$ which is piecewise linear in each component.

Proof. First of all note that we are only interested in an optimal trajectory for $s \in\left[0, s^{*}\right]$ with

$$
s^{*}=\min \left\{\|a\|_{1}:(a, b) \in M_{v e r}^{o p t}\right\} .
$$

Then we can stop since an optimal solution $\left(a\left(s^{*}\right), b\left(s^{*}\right)\right) \in M_{v e r}^{\text {opt }}\left(s^{*}\right)$ to the restricted problem $\left(P_{\text {ver }}\left(s^{*}\right)\right)$ has to be an optimal solution to the unrestricted problem $\left(P_{\text {ver }}\right)$ as well and this means that $\left(a\left(s^{*}\right), b\left(s^{*}\right)\right)$ is optimal for any $\left(P_{\text {ver }}(s)\right)$ with $s>s^{*}$ (see Lemma V.2.1).

Assume that $\mathcal{M}=\left\{\left(a_{1}, b_{1}\right), \ldots,\left(a_{t}, b_{t}\right)\right\}$ is the set of all vertices $(a, b)$ of cells in $\mathcal{C}^{\prime}$ such that $\|a\|_{1} \in\left[0, s^{*}\right]$. This set is finite since there only exist finitely many vertices of cells in $\mathcal{C}^{\prime}$. This is because a vertex of a cell in $\mathcal{C}^{\prime}$ corresponds to the intersection of $k+1$ hyperplanes of the set $\mathcal{H} \cup \mathcal{H}^{\prime \prime}$ and there can only exist finitely many of intersections of $k+1$ hyperplanes since $\mathcal{H}$ and $\mathcal{H}^{\prime \prime}$ are finite sets. Since $\mathcal{M}$ is a subset of the finite set of all vertices of cells in $\mathcal{C}^{\prime}$ it has to be a finite set itself.

Let us assume without loss of generality that

$$
\left\|a_{1}\right\|_{1} \leq\left\|a_{2}\right\|_{1} \leq \ldots \leq\left\|a_{t}\right\|_{1}
$$

Let $s_{1}:=\left\|a_{1}\right\|_{1}, \ldots, s_{t}:=\left\|a_{t}\right\|_{1}$ and let $\mathcal{S}=\left\{s_{1}, \ldots, s_{t}\right\}$. We assume without loss of generality that

$$
s_{1}<s_{2}<\ldots<s_{t} .
$$

We can do that because if $s_{i}=s_{j}$ for some $i, j \in\{1, \ldots, t\}$ we just skip one of them from $\mathcal{S}$ without losing any information.

Note that there exists a $(a, b) \in \mathcal{M}$ with $\|a\|_{1}=0$. This is true because for any optimal solution $(a, b)$ to $\left(P_{\text {ver }}(0)\right)$ it has to hold that $\|a\|_{1}=0$ (otherwise $(a, b)$ is not feasible) and furthermore Theorem V.2.1 tells us that there exists an optimal 


\section{LOCATING HYPERPLANES WITH A RESTRICTION ON THE SLOPE USING VERTICAL DISTANCE

solution to $\left(P_{v e r}(0)\right)$ that passes through at least one of the existing facilities. Therefore this optimal solution is an intersection of the $k$ hyperplanes in $\mathcal{H}^{\prime \prime}$ and one hyperplane $H_{-x_{i}, y_{i}} \in \mathcal{H}$, which means that it is a vertex of a cell in $\mathcal{C}^{\prime}$. Hence we know that $s_{1}=0$.

Moreover, our trajectory has to end in a vertex, in other words there exists a $(a, b) \in \mathcal{M}$ with $\|a\|_{1}=s^{*}$. In order to see that, assume that $\left(a\left(s^{*}\right), b\left(s^{*}\right)\right)$ is not a vertex, then it lies on an edge $e$ of a cell $C^{\prime} \in \mathcal{C}^{\prime}$. There exist $\underline{s}$ and $\bar{s}$ such that $s^{*} \in(\underline{s}, \bar{s})$ and $(a(\underline{s}), b(\underline{s}))$ as well as $(a(\bar{s}), b(\bar{s}))$ are vertices of $C^{\prime}$ delimiting the edge $e$. We can find feasible solutions $(a(s), b(s))$ to $\left(P_{\text {ver }}(s)\right)$ for $s \in\left(s^{*}, \bar{s}\right)$ lying on the same edge $e$ of $C^{\prime}$ as $\left(a\left(s^{*}\right), b\left(s^{*}\right)\right.$. These solutions are optimal to $\left(P_{\text {ver }}(s)\right)$ since they correspond to optimal basic solutions $a^{\prime}(s)$ to $(\tilde{P}(s))$. Since $\left(a\left(s^{*}\right), b\left(s^{*}\right)\right)$ is optimal to $\left(P_{\text {ver }}(s)\right)$ for all $s \geq s^{*}$ (see Lemma V.2.1) and $f_{\text {ver }}$ is linear on $C^{\prime}$, this means that all $(a(s), b(s))$ with $s \in(\underline{s}, \bar{s})$ lying on the edge $e$ are optimal to the unrestricted problem $\left(P_{v e r}\right)$. But this is a contradiction to $s^{*}=\min \left\{\|a\|_{1}:(a, b) \in M_{v e r}^{o p t}\right\}$. Hence we know that $s_{t}=s^{*}$.

Now we have finitely many intervals

$$
\left(s_{1}, s_{2}\right), \ldots,\left(s_{t-1}, s_{t}\right)
$$

such that there exists no vertex $(a, b)$ of a cell in $\mathcal{C}^{\prime}$ with $\|a\|_{1} \in\left(s_{i}, s_{i+1}\right)$ for $s \in\{1, \ldots, t-1\}$. Since we know that $s_{1}=0$ and $s_{t}=s^{*}$, these intervals together with $0, s_{2} \ldots, s_{t-1}, s^{*}$ cover the whole interval $\left[0, s^{*}\right]$ that we are interested in.

Corollary V.3.1 together with our considerations concerning events and vertices of cells in $\mathcal{C}^{\prime}$ assures that on each interval $\left(s_{i}, s_{i+1}\right)$ with $i=1, \ldots, t-1$ we can find $(a(s), b(s)) \in M_{\text {ver }}^{\text {opt }}(s)$ in such a way that $a(s)_{j}$ is linear in $s$ for all $j=1 \ldots k$ as well as $b(s)$ is linear in $s$. There exist only finitely many of these intervals, therefore we can choose any $(a(s), b(s)) \in M_{v e r}^{\text {opt }}(s)$ for $s \in\left(0, s_{2}, \ldots, s^{*}\right)$ in addition and what we get is an optimal trajectory for $\left(P_{v e r}(s)\right)$ which is piecewise linear in each component.

Remark V.3.5. Remember that our starting solution $(a(\tilde{s}), b(\tilde{s}))$ is a vertex of a cell $\tilde{C}$ induced by $\mathcal{H} \cup \mathcal{H}^{\prime} \cup \mathcal{H}^{\prime \prime}$ by assumption (see page 125). This is why we were able to prove that there exists an optimal trajectory that is piecewise linear running along edges of cells $\tilde{C} \in \tilde{\mathcal{C}}$ and in particular passing through $(a(\tilde{s}), b(\tilde{s}))$. 
While this is a nice property of an optimal trajectory from the geometrical point of view, it is important to note that if we drop the assumption of $(a(\tilde{s}), b(\tilde{s}))$ being a vertex of a cell $\tilde{C} \in \tilde{\mathcal{C}}$ we can still prove the existence of a piecewise linear optimal trajectory passing through $(a(\tilde{s}), b(\tilde{s}))$ using similar techniques as the one we presented here. In other words, independent from our choice of a starting solution $(a(\tilde{s}), b(\tilde{s})) \in M_{\text {ver }}^{\text {opt }}(\tilde{s})$ for a fix $\tilde{s}$ we can construct an optimal trajectory that is piecewise linear and at the same time passes through $(a(\tilde{s}), b(\tilde{s}))$.

Finally, we want to use the existence of a piecewise linear optimal trajectory to prove that $F^{*}$ is lower semicontinuous. Then we have everything we need at hand to apply the Michael selection Theorem V.3.1.

Theorem V.3.6. The set-valued map

$$
\begin{aligned}
F^{*}: \mathbb{R}_{0}^{+} & \rightarrow 2^{\mathbb{R}^{k+1}} \\
s & \mapsto M_{\text {ver }}^{\text {opt }}(s)
\end{aligned}
$$

is lower semicontinuous.

Proof. We want to show that $F^{*}$ is lower semicontinuous. Remember that $F^{*}$ is lower semicontinuous in $\hat{s}$ if for any open set $V \in 2^{\mathbb{R}^{k+1}}$ such that $F^{*}(\hat{s}) \cap V \neq \emptyset$ there exists a neighbourhood $U \subseteq \mathbb{R}$ such that $\hat{s} \in U$ and $F^{*}(s) \cap V \neq \emptyset$ for any $s \in U$.

Let $V \in 2^{\mathbb{R}^{k+1}}$ be open such that $F^{*}(\hat{s}) \cap V \neq \emptyset$. In other words, there exists an optimal solution to $\left(P_{\text {ver }}(\hat{s})\right)$ in $V$, i.e. $M_{v e r}^{\text {opt }}(\hat{s}) \cap V \neq \emptyset$. Let $(a(\hat{s}), b(\hat{s})) \in M_{\text {ver }}^{\text {opt }}(\hat{s}) \cap$ $V$. Then there exists an open $\epsilon$-neighbourhood $V_{\epsilon}(a(\hat{s}), b(\hat{s}))$ of $(a(\hat{s}), b(\hat{s}))$ such that $V_{\epsilon}(a(\hat{s}), b(\hat{s})) \subseteq V$.

We want to show that there exists an open neighbourhood $U_{\delta}=(\hat{s}-\delta, \hat{s}+\delta)$ of $\hat{s}$ such that $F^{*}(s) \cap V_{\epsilon}(a(\hat{s}), b(\hat{s})) \neq \emptyset$ for any $s \in U_{\delta}$.

Remember that we can choose $(a(s), b(s)) \in M_{v e r}^{\text {opt }}(s)$ for $s \geq 0$ in a way that the corresponding optimal trajectory is piecewise linear in $s$ and passes through $(a(\hat{s}), b(\hat{s}))$ due to Remark V.3.5. We only want to discuss the case where we start in a vertex $(a(\hat{s}), b(\hat{s})) \in M_{\text {ver }}^{\text {opt }}(s)$ of a cell $\tilde{C}$ induced by $\mathcal{H} \cup \mathcal{H}^{\prime} \cup \mathcal{H}^{\prime \prime}$ here. If $(a(\hat{s}), b(\hat{s}))$ is not a vertex, similar considerations to the one we present here lead to the same result. 


\section{LOCATING HYPERPLANES WITH A RESTRICTION ON THE SLOPE USING VERTICAL DISTANCE

Let $\mathcal{M}=\left\{\left(a_{1}, b_{1}\right), \ldots,\left(a_{t}, b_{t}\right)\right\}$ again be the set of all vertices $(a, b)$ of cells in $\mathcal{C}^{\prime}$ such that $\|a\|_{1} \in\left[0, s^{*}\right]$ and furthermore $s_{1}:=\left\|a_{1}\right\|_{1}, \ldots, s_{t}:=\left\|a_{t}\right\|_{1}$ as well as $\mathcal{S}=\left\{s_{1}, \ldots, s_{t}\right\}$ as in the proof of Theorem V.3.5. Again we assume without loss of generality that

$$
s_{1}<s_{2}<\ldots<s_{t}
$$

We can choose $(a(s), b(s)) \in M_{\text {ver }}^{\text {opt }}(s)$ in a way that $(a(s), b(s))$ is linear in $s$ for $s \in\left(s_{i}, s_{i+1}\right)$ due to Theorem V.3.5. If $(a(\hat{s}), b(\hat{s}))$ is a vertex of a cell $\tilde{C}$ this means that the corresponding optimal trajectory moves along an edge of a certain cell $C_{1} \in \mathcal{C}^{\prime}$ for $s \in\left(s_{i}, s_{i+1}\right), i \in\{1, \ldots, t-1\}$, see Lemma V.3.5.

We have to distinguish between three cases: The easiest case is that $\hat{s} \in\left(s_{i}, s_{i+1}\right)$ for $i \in\{1, \ldots, t-1\}$, in other words there exists no vertex $(a, b) \in \mathcal{M}$ with $\|a\|_{1}=\hat{s}$ and therefore no event takes place in $\hat{s}$.

The second case is that $\hat{s} \in\left\{s_{1}, \ldots, s_{t}\right\}$ but still no event takes place in $\hat{s}$. Geometrically this means that there exists a vertex $(a, b) \in \mathcal{M}$ of a cell $C \in \mathcal{C}^{\prime}$ with $\|a\|_{1}=\hat{s}$ but it is not a vertex of the cell $C_{1}$ we are considering, that is to say $C \neq C_{1}$.

The third case is that $\hat{s} \in\left\{s_{1}, \ldots, s_{t}\right\}$ and an event takes place in $\hat{s}$. The geometrical interpretation of this case is that there exists a vertex $(a, b) \in \mathcal{M}$ of $C_{1} \in \mathcal{C}^{\prime}$ with $\|a\|_{1}=\hat{s}$.

\section{Case 1}

First, let $\hat{s} \in\left(s_{i}, s_{i+1}\right)$ for $i \in\{1, \ldots, t-1\}$. We can choose $\delta$ small enough such that $(\hat{s}-\delta, \hat{s}+\delta) \subseteq\left(s_{i}, s_{i+1}\right)$.

Due to Theorem V.3.5, we can find $(a(s), b(s)) \in M_{\text {ver }}^{\text {opt }}(s)$ such that $(a(s), b(s))$ is linear in $s$ for $s \in(\hat{s}-\delta, \hat{s}+\delta)$. To be more precise, we can find an optimal solution $(a(s), b(s))$ to $\left(P_{v e r}(s)\right)$ with $s \in(\hat{s}-\delta, \hat{s}+\delta)$ such that it has the same active set as well as the same set of passing hyperplanes as $(a(\hat{s}), b(\hat{s}))$ and it holds that

$$
\begin{aligned}
a_{j}(\hat{s}) & =\left\{\begin{array}{l}
(\hat{s}-s) c(j)+a(s)_{j} \text { for } j \in \mathcal{A} \\
a(s)_{j}=0 \text { else }
\end{array}\right. \\
b(\hat{s}) & =(\hat{s}-s) c(b)+b(s)
\end{aligned}
$$


where the $c(j)$ and $c(b)$ are real numbers derived from the entries of the matrix $A$ belonging to the alternative program $(\tilde{P}(\hat{s}))$ defined as on page 127 . By definition of $F^{*}$ it is clear that $(a(s), b(s)) \in F^{*}(s)$. Due to the linearity of $(a(s), b(s))$ it is clear that we can choose $\delta$ small enough such that $(a(s), b(s))$ actually is a point in $V_{\epsilon}(a(\hat{s}), b(\hat{s}))$ for $s \in(\hat{s}-\delta, \hat{s}+\delta)$.

Now we found out that there exists an open neighbourhood $(\hat{s}-\delta, \hat{s}+\delta)$ of $\hat{s}$ such that $F^{*}(s) \cap V_{\epsilon}(a(\hat{s}), b(\hat{s})) \neq \emptyset$ for any $s \in(\hat{s}-\delta, \hat{s}+\delta)$ which means that $F^{*}$ is lower semicontinuous in $\hat{s}$. Since $\hat{s}$ was chosen arbitrarily such that $\hat{s} \in\left(s_{i}, s_{i+1}\right)$ for $i \in\{1, \ldots, t-1\}$ it follows that $F^{*}$ is lower semicontinuous in any $s \in\left(s_{i}, s_{i+1}\right)$ for $i \in\{1, \ldots, t-1\}$.

\section{Case 2}

In this case we assume that $\hat{s} \in\left\{s_{1}, \ldots, s_{t}\right\}$ but still no event takes place in $\hat{s}$. Assume that $\hat{s}=s_{\hat{i}}$ with $\hat{i} \in\{2, \ldots, t-1\}$.

Note that $\hat{s}=s_{1}$ or $\hat{s}=s_{t}$ cannot hold since we already noted in the proof of Theorem V.3.5 that our trajectory has to start and to end in vertices of cells in $\mathcal{C}^{\prime}$. Therefore there exist vertices corresponding to $s_{1}=0$ and to $s_{t}=s^{*}$ that we have to consider.

Let us now look at the interval $\left(s_{\hat{i}-1}, s_{\hat{i}}\right)$. We can choose $(a(s), b(s)) \in M_{\text {ver }}^{\text {opt }}(s)$ such that our trajectory moves along a certain edge $e$ of a cell $C_{1} \in \mathcal{C}^{\prime}$ for $s \in\left(s_{\hat{i}-1}, s_{\hat{i}}\right)$ due to Lemma V.3.5. We know that there exists a vertex $(a, b)$ of a cell $C_{2} \in \mathcal{C}^{\prime}$ such that $\|a\|_{1}=s_{\hat{i}}$ and furthermore $(a, b) \notin e$ by assumption. Therefore there exists another vertex $\left(a\left(s_{i}\right), b\left(s_{i}\right)\right)$ with $\|a\|_{1}=s_{i}>s_{\hat{i}}$ delimiting the edge $e$ for growing $s, s_{i} \in\left\{s_{\hat{i}+1}, \ldots, s_{t}\right\}$. This means that we can find a $\left(a\left(s_{\hat{i}}\right), b\left(s_{\hat{i}}\right)\right)$ lying on the edge $e$ that is feasible for $\left(P_{v e r}\left(s_{\hat{i}}\right)\right)$. Moreover, we can find $(a(s), b(s))$ lying on the edge $e$ feasible for $\left(P_{v e r}(s)\right)$ for all $s \in\left(s_{\hat{i}}, s_{\hat{i}+1}\right)$. Since all of these solutions correspond to optimal basic solutions $a^{\prime}(s)$ to $(\tilde{P}(s)), s \in\left[s_{\hat{i}}, s_{\hat{i}+1}\right)$, they will be optimal to $\left(P_{\text {ver }}(s)\right)$.

Putting this together, we get that we can find $(a(s), b(s)) \in M_{v e r}^{o p t}(s)$ such that $(a(s), b(s))$ is linear in $s$ for $s \in\left(s_{\hat{i}-1}, s_{\hat{i}+1}\right)$ which puts us back to Case 1 . Therefore 
$F^{*}$ is lower semicontinuous in $s_{\hat{i}}$ in particular.

\section{Case 3}

In this case we again assume that $\hat{s} \in\left\{s_{1}, \ldots, s_{t}\right\}$ but this time an event happens in $\hat{s}$.

We first assume that $\hat{s}=s_{\hat{i}}$ with $s_{\hat{i}} \in\left\{s_{2}, \ldots, s_{t-1}\right\}$. Then $\left(a\left(s_{\hat{i}}\right), b\left(s_{\hat{i}}\right)\right)$ is the vertex of a cell $C \in \mathcal{C}^{\prime}$ corresponding to $s_{\hat{i}}$. Let $\delta>0$ be small enough such that $s_{\hat{i}}-\delta \in\left(s_{\hat{i}-1}, s_{\hat{i}}\right)$ as well as $s_{\hat{i}}+\delta \in\left(s_{\hat{i}}, s_{\hat{i}+1}\right)$.

Moreover, let $\mathcal{A}$ and $\mathcal{V}$ be the active set and the set of passing hyperplanes for all $s \in\left(s_{\hat{i}-1}, s_{\hat{i}}\right)$ and let $\mathcal{A}\left(a\left(s_{\hat{i}}\right), b\left(s_{\hat{i}}\right)\right)$ and $\mathcal{V}\left(a\left(s_{\hat{i}}\right), b\left(s_{\hat{i}}\right)\right)$ be the active set and the set of passing hyperplanes for $\left(a\left(s_{\hat{i}}\right), b\left(s_{\hat{i}}\right)\right) \in M_{\text {ver }}^{\text {opt }}\left(s_{\hat{i}}\right)$ such that

$$
\mathcal{A}\left(a\left(s_{\hat{i}}\right), b\left(s_{\hat{i}}\right)\right)=\mathcal{A} \backslash\left\{j^{\prime}\right\} \text { for a } j^{\prime} \in \mathcal{A} \text { and } \mathcal{V}\left(a\left(s_{\hat{i}}\right), b\left(s_{\hat{i}}\right)\right)=\mathcal{V}
$$

or

$$
\mathcal{A}\left(a\left(s_{\hat{i}}\right), b\left(s_{\hat{i}}\right)\right)=\mathcal{A} \text { and } \mathcal{V}\left(a\left(s_{\hat{i}}\right), b\left(s_{\hat{i}}\right)\right)=\mathcal{V} \cup\left\{i^{\prime}\right\} \text { for an } i^{\prime} \notin \mathcal{V}_{\mathcal{H}}
$$

Due to our notion of an event and the one at a time condition we assumed (see Remark V.3.4 $)$, these are the only two possibilities of how $\mathcal{A}\left(a\left(s_{\hat{i}}\right), b\left(s_{\hat{i}}\right)\right)$ and $\mathcal{V}\left(a\left(s_{\hat{i}}\right), b\left(s_{\hat{i}}\right)\right)$ can look like. Note that in either case $\left(a\left(s_{\hat{i}}\right), b\left(s_{\hat{i}}\right)\right)$ corresponds to a feasible solution $a^{\prime}\left(s_{\hat{i}}\right)$ for $\left(\tilde{P}_{\left(s_{\hat{i}-1}, s_{\hat{i}}\right)}\left(s_{\hat{i}}\right)\right)$ where $\left(\tilde{P}_{\left(s_{\hat{i}-1}, s_{\hat{i}}\right)}(s)\right)$ is the alternative linear program defined for $s \in\left(s_{\hat{i}}-\delta, s_{\hat{i}}\right) \subseteq\left(s_{\hat{i}-1}, s_{\hat{i}}\right)$. Furthermore it holds that $f_{\text {ver }}\left(a\left(s_{\hat{i}}\right), b\left(s_{\hat{i}}\right)\right)=c^{t} a^{\prime}\left(s_{\hat{i}}\right)$ by definition of $c$ and $a^{\prime}\left(s_{\hat{i}}\right)$ (see Page 129) and therefore $a^{\prime}\left(s_{\hat{i}}\right)$ is an optimal solution to $\left(\tilde{P}_{\left(s_{\hat{i}-1}, s_{\hat{i}}\right)}\left(s_{\hat{i}}\right)\right)$. Now we can express $\left(a\left(s_{\hat{i}}\right), b\left(s_{\hat{i}}\right)\right)$ as

$$
\begin{aligned}
a_{j}\left(s_{\hat{i}}\right) & =\left\{\begin{array}{l}
\left(s_{\hat{i}}-s\right) c(j)+a(s)_{j} \text { for } j \in \mathcal{A} \backslash\left\{j^{\prime}\right\} \\
a(s)_{j}=0 \text { else }
\end{array}\right. \\
b\left(s_{\hat{i}}\right) & =\left(s_{\hat{i}}-s\right) c(b)+b(s)
\end{aligned}
$$

if $\mathcal{A}\left(a\left(s_{\hat{i}}\right), b\left(s_{\hat{i}}\right)\right)=\mathcal{A} \backslash\left\{j^{\prime}\right\}$ for a $j^{\prime} \in \mathcal{A}$ and $\mathcal{V}\left(a\left(s_{\hat{i}}\right), b\left(s_{\hat{i}}\right)\right)=\mathcal{V}$ or as 


$$
\begin{aligned}
a_{j}\left(s_{\hat{i}}\right) & =\left\{\begin{array}{l}
\left(s_{\hat{i}}-s\right) c(j)+a(s)_{j} \text { for } j \in \mathcal{A} \\
a(s)_{j}=0 \text { else }
\end{array}\right. \\
b\left(s_{\hat{i}}\right) & =\left(s_{\hat{i}}-s\right) c(b)+b(s)
\end{aligned}
$$

if $\mathcal{A}\left(a\left(s_{\hat{i}}\right), b\left(s_{\hat{i}}\right)\right)=\mathcal{A}$ and $\mathcal{V}\left(a\left(s_{\hat{i}}\right), b\left(s_{\hat{i}}\right)\right)=\mathcal{V} \cup\left\{i^{\prime}\right\}$ for an $i^{\prime} \notin \mathcal{V}_{\mathcal{H}}$ using a $s \in$ $\left(s_{\hat{i}}-\delta, s_{\hat{i}}\right)$. Here, the $c(j)$ and $c(b)$ are real numbers derived from the entries of the matrix $A$ belonging to $\left(\tilde{P}_{\left(s_{\hat{i}-1}, s_{\hat{i}}\right)}(s)\right)$.

In other words in either case we can find $(a(s), b(s)) \in M_{v e r}^{\text {opt }}(s)$ such that $(a(s), b(s))$ is linear in $s$ for $s \in\left(s_{\hat{i}}-\delta, s_{\hat{i}}\right]$. Like in Case 1 it holds that $(a(s), b(s)) \in F^{*}(s)$ by definition of $F^{*}$ and due to the linearity of $(a(s), b(s))$ for $s \in\left(s_{\hat{i}}-\delta, s_{\hat{i}}\right)$ we can choose $\delta$ small enough such that $(a(s), b(s))$ actually is a point in $V_{\epsilon}\left(a\left(s_{\hat{i}}\right), b\left(s_{\hat{i}}\right)\right)$ for $s \in\left(s_{\hat{i}}-\delta, s_{\hat{i}}\right)$.

Now we have to check what happens if $s \in\left(s_{\hat{i}}, s_{\hat{i}}+\delta\right) \subseteq\left(s_{\hat{i}}, s_{\hat{i}+1}\right)$. But we can deal with this case analogously to the considerations above: Let now $\mathcal{A}$ and $\mathcal{V}$ be the active set and the set of passing hyperplanes for all $s \in\left(s_{\hat{i}}, s_{\hat{i}}+1\right)$ and let $\mathcal{A}\left(a\left(s_{\hat{i}}\right), b\left(s_{\hat{i}}\right)\right)$ and $\mathcal{V}\left(a\left(s_{\hat{i}}\right), b\left(s_{\hat{i}}\right)\right)$ still be the active set and the set of passing hyperplanes for $\left(a\left(s_{\hat{i}}\right), b\left(s_{\hat{i}}\right)\right) \in M_{\text {ver }}^{\text {opt }}\left(s_{\hat{i}}\right)$. Again we have that

$$
\mathcal{A}\left(a\left(s_{\hat{i}}\right), b\left(s_{\hat{i}}\right)\right)=\mathcal{A} \backslash\left\{j^{\prime}\right\} \text { for a } j^{\prime} \in \mathcal{A} \text { and } \mathcal{V}\left(a\left(s_{\hat{i}}\right), b\left(s_{\hat{i}}\right)\right)=\mathcal{V}
$$

or

$$
\mathcal{A}\left(a\left(s_{\hat{i}}\right), b\left(s_{\hat{i}}\right)\right)=\mathcal{A} \text { and } \mathcal{V}\left(a\left(s_{\hat{i}}\right), b\left(s_{\hat{i}}\right)\right)=\mathcal{V} \cup\left\{i^{\prime}\right\} \text { for an } i^{\prime} \notin \mathcal{V}_{\mathcal{H}}
$$

due to our one at a time assumption. In either case $\left(a\left(s_{\hat{i}}\right), b\left(s_{\hat{i}}\right)\right)$ corresponds to a feasible solution $a^{\prime}\left(s_{\hat{i}}\right)$ for $\left(\tilde{P}_{\left(s_{\hat{i}}, s_{\hat{i}+1}\right)}\left(s_{\hat{i}}\right)\right)$ where $\left(\tilde{P}_{\left(s_{\hat{\imath}}, s_{\hat{i}+1}\right)}(s)\right)$ is the alternative linear program defined for $s \in\left(s_{\hat{i}}, s_{\hat{i}}+\delta\right) \subseteq\left(s_{\hat{i}}, s_{\hat{i}+1}\right)$ and in addition it holds that $f_{\text {ver }}\left(a\left(s_{\hat{i}}\right), b\left(s_{\hat{i}}\right)\right)=c^{t} a^{\prime}\left(s_{\hat{i}}\right)$. Therefore $a^{\prime}\left(s_{\hat{i}}\right)$ is an optimal solution to $\left(\tilde{P}_{\left(s_{\hat{i}}, s_{\hat{i}+1}\right)}\left(s_{\hat{i}}\right)\right)$. 
This time we can express $\left(a\left(s_{\hat{i}}\right), b\left(s_{\hat{i}}\right)\right)$ as

$$
\begin{aligned}
a_{j}\left(s_{\hat{i}}\right) & =\left\{\begin{array}{l}
\left(s-s_{\hat{i}}\right) c(j)+a(s)_{j} \text { for } j \in \mathcal{A} \backslash\left\{j^{\prime}\right\} \\
a(s)_{j}=0 \text { else }
\end{array}\right. \\
b\left(s_{\hat{i}}\right) & =\left(s-s_{\hat{i}}\right) c(b)+b(s)
\end{aligned}
$$

if $\mathcal{A}\left(a\left(s_{\hat{i}}\right), b\left(s_{\hat{i}}\right)\right)=\mathcal{A} \backslash\left\{j^{\prime}\right\}$ for a $j^{\prime} \in \mathcal{A}$ and $\mathcal{V}\left(a\left(s_{\hat{i}}\right), b\left(s_{\hat{i}}\right)\right)=\mathcal{V}$ or as

$$
\begin{aligned}
a_{j}\left(s_{\hat{i}}\right) & =\left\{\begin{array}{l}
\left(s-s_{\hat{i}}\right) c(j)+a(s)_{j} \text { for } j \in \mathcal{A} \\
a(s)_{j}=0 \text { else }
\end{array}\right. \\
b\left(s_{\hat{i}}\right) & =\left(s-s_{\hat{i}}\right) c(b)+b(s)
\end{aligned}
$$

if $\mathcal{A}\left(a\left(s_{\hat{i}}\right), b\left(s_{\hat{i}}\right)\right)=\mathcal{A}$ and $\mathcal{V}\left(a\left(s_{\hat{i}}\right), b\left(s_{\hat{i}}\right)\right)=\mathcal{V} \cup\left\{i^{\prime}\right\}$ for an $i^{\prime} \notin \mathcal{V}_{\mathcal{H}}$ using a $s \in$ $\left(s_{\hat{i}}, s_{\hat{i}}+\delta\right)$. Here, the $c(j)$ and $c(b)$ are real numbers derived from the entries of the matrix $A$ belonging to $\left(\tilde{P}_{\left(s_{\hat{i}}, s_{\hat{i}+1}\right)}(s)\right)$.

So this time we can find $(a(s), b(s)) \in M_{v e r}^{\text {opt }}(s)$ such that $(a(s), b(s))$ is linear in $s$ for $s \in\left[s_{\hat{i}}, s_{\hat{i}}+\delta\right)$. This leads to the conclusion that we can choose $\delta$ small enough such that $(a(s), b(s))$ actually is a point in $V_{\epsilon}\left(a\left(s_{\hat{i}}\right), b\left(s_{\hat{i}}\right)\right)$ for $s \in\left(s_{\hat{i}}, s_{\hat{i}}+\delta\right)$.

Summarizing our results so far we get that there exists an open neighbourhood $\left(s_{\hat{i}}-\delta, s_{\hat{i}}+\delta\right)$ of $s_{\hat{i}}$ such that $F^{*}(s) \cap V_{\epsilon}\left(a\left(s_{\hat{i}}\right), b\left(s_{\hat{i}}\right)\right) \neq \emptyset$ for any $s \in\left(s_{\hat{i}}-\delta, s_{\hat{i}}+\delta\right)$ which means that $F^{*}$ is lower semicontinuous in $s_{\hat{i}}$.

We have chosen $\hat{s}=s_{\hat{i}} \in\left\{s_{2}, \ldots, s_{t-1}\right\}$ arbitrarily, therefore $F^{*}$ is lower semicontinuous in any $s \in\left\{s_{2}, \ldots, s_{t-1}\right\}$.

What is left to check is whether $F^{*}$ is lower semicontinuous in $\hat{s}=s_{1}$ and $\hat{s}=s_{t}$. Since our optimal trajectory starts in $s_{1}=0$ it is sufficient to show that there exists a $\delta$ such that $\left[s_{1}, s_{1}+\delta\right)$ is an open neighbourhood of $s_{1}$ with $F^{*}(s) \cap$ $V_{\epsilon}\left(a\left(s_{1}\right), b\left(s_{1}\right)\right) \neq \emptyset$ for any $s \in\left[s_{1}, s_{1}+\delta\right)$. This can be done analogously to the second part of the considerations concerning the case $\hat{s} \in\left\{s_{2}, \ldots, s_{t-1}\right\}$ above.

Furthermore our optimal trajectory ends in $s_{t}=s^{*}$, therefore it is sufficient to show that there exists a $\delta$ such that $\left(s_{t}-\delta, s_{t}\right]$ is an open neighbourhood of $s_{t}$ with 


\section{V.3. THE EXISTENCE OF A CONTINUOUS OPTIMAL}

$F^{*}(s) \cap V_{\epsilon}\left(a\left(s_{t}\right), b\left(s_{t}\right)\right) \neq \emptyset$ for any $s \in\left(s_{t}-\delta, s_{t}\right]$. This can be done analogously to the first part of the considerations concerning the case $\hat{s} \in\left\{s_{2}, \ldots, s_{t-1}\right\}$ above.

Putting all considerations together we showed that $F^{*}$ is lower semicontinuous in $s \geq 0$.

Remark V.3.6. It is true that we showed more than just the lower semicontinuity of $F^{*}$. In fact, we already showed that there exists a continuous optimal trajectory during the proof of Theorem V.3.6.

First of all we showed in Case 2 that there exist $s \in \mathcal{S}$ such that the corresponding vertex is not of interest concerning our optimal trajectory. Therefore we need not to consider these vertices any longer and we can skip the corresponding s-values from the set $\mathcal{S}$. What we get now is a set $\mathcal{S}^{\prime}=\left\{s_{1}, \ldots, s_{q}\right\}$ of $s$-values such that the vertices corresponding to these values are the only vertices of cells in $\mathcal{C}^{\prime}$ lying on the optimal trajectory we want to consider. In other words, the set $\mathcal{S}^{\prime}$ gives us all s-values in which an event takes place. It is important to note that it is still $s_{1}=0$ and $s_{q}=s^{*}$ which means that our trajectory starts and ends in a vertex of a cell in $\mathcal{C}^{\prime}$. This is equivalent to $s \notin\left\{s_{1}, s_{t}\right\}$ in Case 2.

We already knew that we can find $(a(s), b(s)) \in M_{v e r}^{\text {opt }}(s)$ for $s \in\left(s_{i}, s_{i+1}\right), i \in$ $\{1, \ldots, q-1\}$, such that $(a(s), b(s))$ is linear in $s$ (see Theorem V.3.5). What we did during the proof is to show that there exists a $\left(a\left(s_{i}\right), b\left(s_{i}\right)\right)$ for $s_{i} \in\left\{s_{2}, \ldots, s_{q-1}\right\}$ such that $\left(a\left(s_{i}\right), b\left(s_{i}\right)\right)$ can be derived from an optimal solution for $\left(\tilde{P}_{\left(s_{i-1}, s_{i}\right)}(s)\right)$ as well as from an optimal solution for $\left(\tilde{P}_{\left(s_{i}, s_{i+1}\right)}(s)\right)$. This tells us that for $s \nearrow s_{i}$ as well as for $s \searrow s_{i}$ it holds that $(a(s), b(s)) \longrightarrow\left(a\left(s_{i}\right), b\left(s_{i}\right)\right)$ for $i=2, \ldots, q-1$. Furthermore $s_{1}$ and $s_{q}$ can be derived from optimal solutions for $\left(\tilde{P}_{\left(s_{1}, s_{2}\right)}(s)\right)$ and $\left(\tilde{P}_{\left(s_{q-1}, s_{q}\right)}(s)\right)$, respectively, providing that

$$
(a(s), b(s)) \longrightarrow\left(a\left(s_{1}\right), b\left(s_{1}\right)\right) \text { for } s \searrow s_{1}
$$

and

$$
(a(s), b(s)) \longrightarrow\left(a\left(s_{q}\right), b\left(s_{q}\right)\right) \text { for } s \nearrow s_{q}
$$

Putting this together we have that the $(a(s), b(s))$ corresponding to an optimal solution $a^{\prime}(s)$ to $\left(\tilde{P}_{\left(s_{i}, s_{i+1}\right)}(s)\right)$ for $s \in\left(s_{i}, s_{i+1}\right), i=1, \ldots, q-1$, together with the 
$(a(s), b(s))$ chosen as in the proof of Theorem $V$.3.6 for $s \in \mathcal{S}^{\prime}=\left\{s_{1}, s_{2}, \ldots, s_{q}\right\}$ give us a continuous optimal trajectory for our problem $\left(P_{\text {ver }}(s)\right), s \geq 0$. Geometrically, our continuous optimal trajectory starts in a vertex of a cell $C_{1} \in \mathcal{C}^{\prime}$, from there it moves along an edge of $C_{1}$ that is connected to this vertex until it reaches another vertex where it changes to an edge of a cell $C_{2} \in \mathcal{C}^{\prime}$ connected to this vertex and so on. We will come back to the geometrical properties of our optimal trajectory in the next section.

Note that the existence of a continuous optimal trajectory does not necessarily imply that $F^{*}$ in Theorem V.3.6 is continuous. If we were interested in continuity of $F^{*}$ we would have to check whether $F^{*}$ is upper semicontinuous in addition to the lower semicontinuity.

For completeness of this section we want to state the following proposition which approves the existence of a continuous optimal trajectory.

Proposition V.3.1. There exists an optimal trajectory $t$,

$$
\begin{aligned}
t: \mathbb{R}_{0}^{+} & \rightarrow \mathbb{R}^{k+1} \\
s & \mapsto(a(s), b(s)),
\end{aligned}
$$

of $\left(P_{\text {ver }}(s)\right), s \geq 0$, that is continuous in $s$.

Proof. Since we can understand a trajectory as a selection of the set-valued map $F^{*}$ and because we used the main part of this section to show that the Michael selection theorem can be applied in our case, the proposition follows directly from Theorem V.3.1

\section{V.4 How to construct a continuous optimal tra- jectory}

In this section we want to show how a continuous optimal trajectory for our problem $\left(P_{\text {ver }}(s)\right)$ with $s \geq 0$ can be constructed. The previous section provides us 


\section{V.4. HOW TO CONSTRUCT A CONTINUOUS OPTIMAL}

everything we need to construct such a trajectory.

As in the two-dimensional case, we start with $s=0$. We know that there exists an optimal solution to $\left(P_{\text {ver }}(0)\right)$ such that the hyperplane in the initial space corresponding to it passes through at least one of the existing facilities (see Theorem V.2.1). Furthermore we know that any optimal solution has to satisfy the pseudohalving property (see Theorem $\overline{V .2 .2}$ ). Therefore an optimal solution must be of the form $(0, \ldots, 0, b)$ such that $b$ is a median of the set $\left\{y_{i}: i=1 \ldots n\right\}$.

If the number of different $y$-values is odd we start with $(0, \ldots, 0, b(0))$ such that $b(0)$ is the unique median of the set $\left\{y_{1}, \ldots, y_{n}\right\}$ as our unique optimal solution.

If the number of different $y$-values is even there might exist more than one optimal solution since there might exist more than one median. Any $(0, \ldots, 0, b)$ such that $b$ is a median of the set $\left\{y_{1}, \ldots, y_{n}\right\}$ is an optimal solution because all these $(0, \ldots, 0, b)$ share the same objective function value. In this case, we choose $(0, \ldots, 0, b(0))$ to be the optimal solution for $\left(P_{v e r}(0)\right)$ such that $b(s)$ is the lower median of the set $\left\{y_{1}, \ldots, y_{n}\right\}$. Note that this again is a unique choice.

In the following, we want to let $s$ grow and give a solution for any $\left(P_{v e r}(s)\right)$ until we reach $s^{*}$ such that we get an optimal solution $\left(a\left(s^{*}\right), b\left(s^{*}\right)\right)$ for $\left(P_{v e r}\left(s^{*}\right)\right)$ which is at the same time an optimal solution for the unrestricted problem $\left(P_{v e r}\right)$. Then we can stop because this $\left(a\left(s^{*}\right), b\left(s^{*}\right)\right)$ is an optimal solution for any $\left(P_{v e r}(s)\right)$ with $s>s^{*}$ as well (see Lemma V.2.1).

Knowing our starting point of the trajectory $(0, \ldots, 0, b(0))$ we can give the set of passing hyperplanes and the active set of this point. We know that the corresponding hyperplane in the initial space passes through at least one of the existing facilities. For the time being, let us assume that it passes through exactly one. This means that in the dual space there is exactly one hyperplane $H_{-x_{i^{\prime}}, y_{i^{\prime}}}$ passing through the point $(0, \ldots, 0, b(0))$, namely the one such that $y_{i^{\prime}}$ is the median or the lower median of the set $\left\{y_{i}: i=1 \ldots n\right\}$, respectively. Therefore the set of passing hyperplanes is $\mathcal{V}(0, b(0))=\left\{i^{\prime}, n+1, \ldots, n+k\right\}$ which we can split up into the set of passed facilities $\mathcal{V}_{\mathcal{H}}(0, b(0))=\left\{i^{\prime}\right\}$ and the inactive set $\mathcal{V}_{\mathcal{H}^{\prime \prime}}(0, b(0))=\{n+1, \ldots, n+k\}$. Note that $(0, \ldots, 0, b(0))$ is a vertex of a cell in $\mathcal{C}^{\prime}$ since it is the intersection of $k+1$ hyperplanes in $\mathcal{H} \cup \mathcal{H}^{\prime \prime}$.

The active set is even more easy to name. Since $a(0)_{j}=0$ for all $j=1, \ldots, k$ the 
active set obviously is the empty set, $\mathcal{A}(0, b(0))=\emptyset$. Note that $\left|\mathcal{V}_{\mathcal{H}}(0, b(0))\right|=1$ while $|\mathcal{A}(0, b(0))|=0$. During the construction of the trajectory we will mainly focus on the active set $\mathcal{A}$ and the set of passed facilities $\mathcal{V}_{\mathcal{H}}$ in the following since the inactive set can always be derived directly from the active set due to Remark V.3.1

If we let $s$ become non-zero, it makes perfect sense that some of the $a(s)_{j}$ will become non-zero as well. In fact, exactly one of the $a(s)_{j}$ will become non-zero, i.e. $\mathcal{A}(a(s), b(s))=\left\{j^{\prime}\right\}$ for some $j^{\prime} \in\{1, \ldots, k\}$, and the set of passed facilities will stay constant, that is $\mathcal{V}_{\mathcal{H}}(0, b(0))=\mathcal{V}_{\mathcal{H}}(a(s), b(s))$, such that $\left|\mathcal{V}_{\mathcal{H}}(a(s), b(s))\right|=$ $|\mathcal{A}(a(s), b(s))|$ holds. This is due to Lemma V.3.3. As long as $\mathcal{A}(a(s), b(s))=\left\{j^{\prime}\right\}$ and $\mathcal{V}_{\mathcal{H}}(a(s), b(s))=\left\{i^{\prime}\right\}$, we know that there exists an optimal solution $(a(s), b(s))$ of $\left(P_{\text {ver }}(s)\right)$ such that

$$
\begin{aligned}
& \operatorname{sgn}\left(a_{j^{\prime}}(s)\right) a_{j^{\prime}}(s)=s \text { and } \\
& a_{j^{\prime}}(s) x_{i^{\prime} j^{\prime}}+b(s)=y_{i^{\prime}} .
\end{aligned}
$$

This is due to Lemma V.3.4. We know that $a_{j^{\prime}}(s)$ and $b(s)$ are both linear in $s$ as long as $\mathcal{V}$ and $\mathcal{A}$ are constant due to Theorem V.3.3. If we write $\Delta s$ instead of $s$ to indicate that we change our current $s$ which is in fact $s=0$ by $\Delta s$, i.e. to $s=0+\Delta s$, we can write $a_{j^{\prime}}(s)$ and $b(s)$ as

$$
\begin{aligned}
a_{j^{\prime}}(s) & =a_{j^{\prime}}(0)+\frac{d a_{j^{\prime}}}{d s} \cdot \Delta s \\
& =\frac{d a_{j^{\prime}}}{d s} \cdot \Delta s
\end{aligned}
$$

and

$$
\begin{aligned}
b(s) & =b(0)+\frac{d b}{d s} \Delta s \\
& =y_{i^{\prime}}+\frac{d b}{d s} \Delta s,
\end{aligned}
$$

respectively, where the derivatives $\frac{d a_{j^{\prime}}}{d s}$ and $\frac{d b}{d s}$ can be derived from the following 
system of equations

$$
\begin{gathered}
\operatorname{sgn}\left(a_{j^{\prime}}(s)\right) \frac{d a_{j^{\prime}}}{d s}=1 \\
\frac{d a_{j^{\prime}}}{d s} x_{i^{\prime} j^{\prime}}+\frac{d b}{d s}=0 .
\end{gathered}
$$

Therefore we know how an optimal solution to $\left(P_{\text {ver }}(s)\right)$ looks like until an event happens. As we already mentioned, an event can happen when a coefficient in the active set becomes zero or an additional hyperplane joins the set of passed facilities (see Remark V.3.4), in other words when an additional hyperplane in $\mathcal{H} \cup \mathcal{H}^{\prime \prime}$ intersects with the hyperplanes that are already corresponding to an index in the set of passing hyperplanes.

Up to now, only $a_{j^{\prime}}(s)$ is active. Let $\Delta s^{1}$ give the change of $s$ such that $a_{j^{\prime}}(s)$ becomes zero again. It is easy to see that $a_{j^{\prime}}(s)$ only becomes zero if $\Delta s^{1}=0$ which cannot be the case since we want to let $s$ grow constantly. Therefore we set $\Delta s^{1}$ to $\infty$ which means that $a_{j^{\prime}}(s)$ increases with growing $s$ if $\operatorname{sgn}\left(a_{j^{\prime}}(s)\right)=1$ or decreases with growing $s$ if $\operatorname{sgn}\left(a_{j^{\prime}}(s)\right)=-1$, respectively, and it will never become zero.

This means that the only event that can happen here is that a new hyperplane joins $\mathcal{V}_{\mathcal{H}}$. A hyperplane $H_{-x_{i}, y_{i}}, i \in\{1, \ldots, n\} \backslash\left\{i^{\prime}\right\}$ joins $\mathcal{V}_{\mathcal{H}}$ if it passes $(a(\Delta s), b(\Delta s))$, that is if the residual for $i$

$$
\operatorname{res}_{i}(a(\Delta s), b(\Delta s)):=\operatorname{res}\left((a(\Delta s), b(\Delta s)), H_{-x_{i}, y_{i}}\right)=y_{i}-\left(a(\Delta s)_{j^{\prime}} x_{i j^{\prime}}+b(\Delta s)\right)
$$

becomes zero. Note that we can determine the derivatives of the residuals as long as $\mathcal{A}$ and $\mathcal{V}$ are constant using the derivatives $\frac{d a_{j^{\prime}}}{d s}$ and $\frac{d b}{d s}$ :

$$
\begin{aligned}
& \operatorname{res}_{i}(a(\Delta s), b(\Delta s)) \\
& =y_{i}-\left(\left(a_{j^{\prime}}(0)+\frac{d a_{j^{\prime}}}{d s} \cdot \Delta s\right) x_{i j^{\prime}}+\left(b(0)+\frac{d b}{d s} \cdot \Delta s\right)\right) \\
& =\operatorname{res}_{i}(a(0), b(0))-\left(\frac{d a_{j^{\prime}}}{d s} \cdot \Delta s \cdot x_{i j^{\prime}}+\frac{d b}{d s} \cdot \Delta s\right)
\end{aligned}
$$


and therefore

$$
\frac{d r e s_{i}}{d s}=-\left(\frac{d a_{j^{\prime}}}{d s} \cdot x_{i j^{\prime}}+\frac{d b}{d s}\right)
$$

Now the residual of $i$ becomes zero if

$$
\Delta s_{i}^{2}=\frac{r e s_{i}(a(0), b(0))}{\left(\frac{d a_{j^{\prime}}}{d s} \cdot x_{i j^{\prime}}+\frac{d b}{d s}\right)}
$$

and we define $\Delta s^{2}$ as

$$
\Delta s^{2}=\min \left\{\Delta s_{i}^{2}: i \notin \mathcal{V}\right\}
$$

If $\Delta s_{i}^{2}<0$ for one $i$ we set $\Delta s_{i}^{2}=\infty$ since we want to let $s$ grow constantly.

We can now determine the stepsize $\Delta s$ until the first event happens:

$$
\Delta s=\min \left\{\Delta s^{1}, \Delta s^{2}\right\}
$$

As we already mentioned, when we start in $s=0$ the stepsize will be $\Delta s=\Delta s^{2}$.

As soon as we know this, we can give a solution to $\left(P_{v e r}(s)\right)$ for any $s \in[0, \Delta s]$, namely $(a(s), b(s))$ with

$$
a_{j}(s)=\left\{\begin{array}{l}
\frac{d a_{j^{\prime}}}{d s} \cdot \Delta s \text { if } j=j^{\prime} \\
0 \text { else }
\end{array}\right.
$$

and

$$
b(s)=y_{i^{\prime}}+\frac{d b}{d s} \Delta s .
$$

Therefore, if we want to know what the trajectory looks like when we let $s$ grow, we have to find out which one of the $a_{j}$ becomes non-zero and what sign it is going to have. Remember that we can write the objective function $f_{v e r}$ as 


$$
\begin{aligned}
f_{v e r}(a, b)= & \sum_{i \in R(a, b)} y_{i}-\sum_{i \in L(a, b)} y_{i} \\
& +a_{1}\left(\sum_{i \in L(a, b)} x_{i 1}-\sum_{i \in R(a, b)} x_{i 1}\right) \\
& +a_{2}\left(\sum_{i \in L(a, b)} x_{i 2}-\sum_{i \in R(a, b)} x_{i 2}\right) \\
& +\ldots \\
& +a_{k}\left(\sum_{i \in L(a, b)} x_{i k}-\sum_{i \in R(a, b)} x_{i k}\right) \\
& +\sum_{i \in L(a, b)} b-\sum_{i \in R(a, b)} b .
\end{aligned}
$$

For $s=0$ this gives us

$$
\begin{aligned}
f_{\text {ver }}(a(0), b(0))= & \sum_{i \in R} y_{i}-\sum_{i \in L} y_{i} \\
& +\sum_{i \in L} b(0)-\sum_{i \in R} b(0) \\
= & \sum_{i \in R} y_{i}-\sum_{i \in L} y_{i} \\
& +|L| \cdot y_{i^{\prime}}-|R| \cdot y_{i^{\prime}} .
\end{aligned}
$$

We only write $R$ and $L$ here instead of $R(a(0), b(0))$ and $L(a(0), b(0))$, respectively, because the sets $R(a(s), b(s))$ and $L(a(s), b(s))$ are constant as long as $s \in[0, \Delta s]$ (see Lemma V.3.1).

Since we want to minimize our objective function, we have to find out how the value $f_{\text {ver }}$ changes if one $a_{j}$ becomes non-zero. We already know that $\mathcal{V}_{\mathcal{H}}=\left\{i^{\prime}\right\}$ with $i^{\prime}$ being the (lower) median of $\left\{y_{i}: i=1, \ldots, n\right\}$ and because we keep the set of passing hyperplanes constant, it must hold that

$$
y_{i^{\prime}}=a_{j}(s) \cdot x_{i^{\prime} j}+b(s)
$$


for $s \in[0, \Delta s]$ if $a_{j}$ becomes non-zero. Therefore the new $b$-value at $s$ is

$$
\begin{aligned}
b(s) & =y_{i^{\prime}}-a_{j}(s) \cdot x_{i^{\prime} j} \\
& =b(0)-a_{j}(s) \cdot x_{i^{\prime} j} .
\end{aligned}
$$

This together with the fact that $R$ and $L$ are constant on $[0, \Delta s]$ enables us to write the new objective function value at $s$ as

$$
\begin{aligned}
f\left(a_{j}(s), b(s)\right)= & \sum_{i \in R} y_{i}-\sum_{i \in L} y_{i} \\
& +a_{j}(s)\left(\sum_{i \in L} x_{i j}-\sum_{i \in R} x_{i j}\right) \\
& +|L| \cdot\left(b(0)-a_{j}(s) \cdot x_{i^{\prime} j}\right)-|R| \cdot\left(b(0)-a_{j}(s) \cdot x_{i^{\prime} j}\right) \\
= & f_{v e r}(a(0), b(0)) \\
& +a_{j}(s) \cdot\left(\sum_{i \in L} x_{i j}-\sum_{i \in R} x_{i j}+(|R|-|L|) \cdot x_{i^{\prime} j}\right) .
\end{aligned}
$$

Note that the expression $\sum_{i \in L} x_{i j}-\sum_{i \in R} x_{i j}+(|R|-|L|) \cdot x_{i^{\prime} j}$ gives us the change of the value of $f_{v e r}$ in $s$ if $a_{j}$ becomes non-zero, that is

$$
\frac{d f_{v e r}\left(a_{j}(s), b(s)\right)}{d s}=\sum_{i \in L} x_{i j}-\sum_{i \in R} x_{i j}+(|R|-|L|) \cdot x_{i^{\prime} j}
$$

if $s \in[0, \Delta s]$.

Remember that we assumed that there exists exactly one hyperplane corresponding to an existing facility passing trough $(0, \ldots, 0, b(0))$. If this is not the case, that is to say there is more than one such hyperplane that passes $(0, \ldots, 0, b(0))$, we first have to calculate $\left(\frac{d f_{v e r}\left(a_{j}(s), b(s)\right)}{d s}\right)_{i}$ for each index $i$ corresponding to a hyperplane passing through $(0, \ldots, 0, b(0))$ by setting $\mathcal{V}_{\mathcal{H}}=\{i\}$ and then we choose $i^{\prime}$ as

$$
\arg \min _{i}\left\{\left(\frac{d f_{\text {ver }}\left(a_{j}(s), b(s)\right)}{d s}\right)_{i}\right\}
$$

and go on as described above. 
Our aim was to choose some $j^{\prime}$ and the sign of $a_{j^{\prime}}$ such that the objective function is minimized. In order to do so, we have to choose $j^{\prime}$ as

$$
j^{\prime}:=\underset{j \in\{1, \ldots, k\}}{\arg \max }\left|\sum_{i \in L} x_{i j}-\sum_{i \in R} x_{i j}+(|R|-|L|) \cdot x_{i^{\prime} j}\right|
$$

and the sign of $a_{j^{\prime}}$ as

$$
\operatorname{sgn}\left(a_{j^{\prime}}(s)\right)=-\operatorname{sgn}\left(\sum_{i \in L} x_{i j^{\prime}}-\sum_{i \in R} x_{i j^{\prime}}+(|R|-|L|) \cdot x_{i^{\prime} j^{\prime}}\right) .
$$

Then we can compute solutions $(a(s), b(s))$ with active set $\mathcal{A}=\left\{j^{\prime}\right\}$ and set of passed facilities $\mathcal{V}_{\mathcal{H}}=\left\{i^{\prime}\right\}$ for $s \in[0, \Delta s]$ by computing $\frac{d a_{j^{\prime}}}{d s}, \frac{d b}{d s}$ and $\Delta s$ as we described above. Additionally we know that the change of the value of $f_{v e r}$ is

$$
\frac{d f_{v e r}(a(s), b(s))}{d s}=\sum_{i \in L} x_{i j^{\prime}}-\sum_{i \in R} x_{i j^{\prime}}+(|R|-|L|) \cdot x_{i^{\prime} j^{\prime}} .
$$

This tells us how an optimal trajectory looks like on the interval $[0, \Delta s]$ until a first event happens. Due to our one at a time condition (see Remark V.3.4), one of the residuals becomes zero which means that the corresponding index $i^{\prime \prime} \notin \mathcal{V}_{\mathcal{H}}$ joins the set of passing hyperplanes. Note that we already mentioned that the first event cannot be $a_{j^{\prime}}(s)$ becoming zero. If there are more than just one indices in the active set an event can of course be that one of the active coefficients become zero again.

Now we get a new set of passed facilities, namely $\mathcal{V}_{\mathcal{H}}^{\text {new }}=\left\{i^{\prime}, i^{\prime \prime}\right\}$, while the active set stays constant, that is $\mathcal{A}^{\text {new }}=\mathcal{A}=\left\{j^{\prime}\right\}$. Therefore it holds that $\left|\mathcal{V}_{\mathcal{H}}^{\text {new }}\right|=$ $\left|\mathcal{A}^{\text {new }}\right|+1$, so in the next step one hyperplane has to leave $\mathcal{V}_{\mathcal{H}}$ or a new index $j^{\prime \prime}$ has to enter the active set $\mathcal{A}$, in other words an additional coefficient $a_{j^{\prime \prime}}(s)$ has to become non-zero. This is due to Lemma V.3.3 in the previous section.

The next task is now to find out which of the hyperplanes should leave the set of passed facilities or which of the coefficients should become non-zero in order to obtain optimal solutions of $\left(P_{v e r}(s)\right)$ on another interval $\left[\Delta s, \Delta s+\Delta s_{\text {new }}\right]$. In order to make this decision, we can compute the change of the objective function value with changing $s$ for any possible variant and then choose the one which is 
the best option.

Let us first consider the case in which one of the hyperplanes leaves $\mathcal{V}_{\mathcal{H}}$, that is either $i^{\prime}$ or $i^{\prime \prime}$ has to leave $\mathcal{V}_{\mathcal{H}}$. Denote by $\hat{i}$ the one coordinate that has to leave the set of passed facilities, $\hat{i} \in\left\{i^{\prime}, i^{\prime \prime}\right\}$. In either case we know that $\frac{d a_{j^{\prime}}}{d s}= \pm 1$ depending on the sign of $a_{j^{\prime}}(s)$ and we can calculate $\frac{d b}{d s}$ by solving the following system of equations:

$$
\begin{aligned}
\operatorname{sgn}\left(a_{j^{\prime}}(s)\right) \frac{d a_{j^{\prime}}}{d s} & =1 \\
\frac{d a_{j^{\prime}}}{d s} x_{\hat{i} j^{\prime}}+\frac{d b}{d s} & =0 .
\end{aligned}
$$

It is easy to see that the new function value is

$$
\begin{aligned}
f_{\text {ver }}(a(s), b(s))= & f_{\text {ver }}(a(\Delta s), b(\Delta s)) \\
& +\Delta s_{\text {new }} \cdot\left(\frac{d a_{j^{\prime}}}{d s} \cdot\left(\sum_{i \in L} x_{i j^{\prime}}-\sum_{i \in R} x_{i j^{\prime}}\right)+(|L|-|R|) \cdot \frac{d b}{d s}\right)
\end{aligned}
$$

In this case we get

$$
\frac{d f_{v e r}(a(s), b(s))}{d s}=\frac{d a_{j^{\prime}}}{d s} \cdot\left(\sum_{i \in L} x_{i j^{\prime}}-\sum_{i \in R} x_{i j^{\prime}}\right)+(|L|-|R|) \cdot \frac{d b}{d s}
$$

Now we consider the case in which an additional coefficient $j^{\prime \prime}$ becomes non-zero. The set of passed facilities is $\mathcal{V}_{\mathcal{H}}=\left\{i^{\prime}, i^{\prime \prime}\right\}$ constantly. Any $j \in \mathcal{A}^{c}$ is a candidate to become non-zero. If we want to check how $f_{\text {ver }}$ changes for $a_{j}(s)$ becoming non-zero we have to consider two cases, namely the case $\operatorname{sgn}\left(a_{j}(s)\right)=1$ and the one with $\operatorname{sgn}\left(a_{j}(s)\right)=-1$. We know the sign of $a_{j^{\prime}}(s)$ since $a_{j^{\prime}}(s)$ is piecewise linear in $s$. Therefore we can calculate $\frac{d a_{j^{\prime}}}{d s}, \frac{d a_{j}}{d s}$ and $\frac{d b}{d s}$ in both cases by solving the system of equations 


$$
\begin{aligned}
\operatorname{sgn}\left(a_{j^{\prime}}(s)\right) \frac{d a_{j^{\prime}}}{d s}+\operatorname{sgn}\left(a_{j}(s)\right) \frac{d a_{j}}{d s} & =1 \\
\frac{d a_{j^{\prime}}}{d s} x_{i^{\prime} j^{\prime}}+\frac{d a_{j}}{d s} x_{i^{\prime} j}+\frac{d b}{d s} & =0 \\
\frac{d a_{j^{\prime}}}{d s} x_{i^{\prime \prime} j^{\prime}}+\frac{d a_{j}}{d s} x_{i^{\prime \prime} j}+\frac{d b}{d s} & =0 .
\end{aligned}
$$

But then we get that

$$
\begin{aligned}
f_{v e r}(a(s), b(s))= & f_{v e r}(a(\Delta s), b(\Delta s))+\Delta s_{\text {new }} \cdot\left(\frac{d a_{j^{\prime}}}{d s}\left(\sum_{i \in L} x_{i j^{\prime}}-\sum_{i \in R} x_{i j^{\prime}}\right)\right. \\
& \left.+\frac{d a_{j}}{d s}\left(\sum_{i \in L} x_{i j}-\sum_{i \in R} x_{i j}\right)+(|L|-|R|) \cdot \frac{d b}{d s}\right)
\end{aligned}
$$

and therefore

$$
\begin{aligned}
\frac{d f_{v e r}(a(s), b(s))}{d s}= & \frac{d a_{j^{\prime}}}{d s}\left(\sum_{i \in L} x_{i j^{\prime}}-\sum_{i \in R} x_{i j^{\prime}}\right)+\frac{d a_{j}}{d s}\left(\sum_{i \in L} x_{i j^{\prime \prime}}-\sum_{i \in R} x_{i j^{\prime \prime}}\right) \\
& +(|L|-|R|) \cdot \frac{d b}{d s}
\end{aligned}
$$

If we have computed $\frac{d f_{v e r}(a(s), b(s))}{d s}$ for each possible case we look at the minimum of all these derivatives of $f_{v e r}$. Two different cases can occur here.

\section{Case 1 (no improvement)}

If the minimum is a positive number or zero, none of the possible variants leads to an improvement of our objective function value. That means that $(a(\Delta s), b(\Delta s))$ is not only an optimal solution for $\left(P_{v e r}(\Delta s)\right)$ but also for $\left(P_{v e r}\right)$ at the same time and therefore we are done because we know a continuous optimal trajectory on the interval $[0, \Delta s]$ which is indeed the whole optimal trajectory. 


\section{LOCATING HYPERPLANES WITH A RESTRICTION ON THE SLOPE USING VERTICAL DISTANCE

\section{Case 2 (improvement)}

If the minimum derivative is negative, we know which event actually takes place in order to get optimal solutions for $\left(P_{\text {ver }}(s)\right)$ with $s \in\left[\Delta s, \Delta s+\Delta s_{\text {new }}\right]$.

If the cardinality of $\mathcal{A}$ and the one of $\mathcal{V}_{\mathcal{H}}$ is one in the optimal case, we know $\frac{d a_{j^{\prime}}}{d s}$ and $\frac{d b}{d s}$ and can compute the derivatives of all residuals res $_{i}$ with $i \notin \mathcal{V}_{\mathcal{H}}$ as we did above:

$$
\frac{d r e s_{i}}{d s}=-\left(\frac{d a_{j^{\prime}}}{d s} \cdot x_{i j^{\prime}}+\frac{d b}{d s}\right) .
$$

In the end we can again calculate $\Delta s_{\text {new }}^{1}$ and $\Delta s_{\text {new }}^{2}$ and we get that $\Delta s_{\text {new }}^{1}=\infty$ and

$$
\Delta s^{2}=\min \left\{\Delta s_{\text {new }}^{2}: i \notin \mathcal{V}\right\}
$$

where

$$
\Delta s_{\text {new }}^{2}=\frac{\operatorname{res}_{i}(a(\Delta s), b(\Delta s))}{-\frac{d r e s}{d s}}
$$

Note that in this case $\Delta s^{2}$ cannot be $\infty$ because that would mean that we do not get an improvement of $f_{\text {ver }}$ which we already handled in Case 1. Now we finally get $\Delta s_{\text {new }}$ as

$$
\Delta s_{\text {new }}=\min \left\{\Delta s_{\text {new }}^{1}, \Delta s_{\text {new }}^{2}\right\}
$$

and we know how an optimal trajectory looks like on the interval $\left[\Delta s, \Delta s_{\text {new }}\right]$.

If on the other hand the cardinality of $\mathcal{A}$ and $\mathcal{V}_{\mathcal{H}}$ is two now in the optimal case, we get slightly different computations. We know $\frac{d a_{j^{\prime}}}{d s}, \frac{d a_{j^{\prime \prime}}}{d s}$ and $\frac{d b}{d s}$. In this case, it is possible that the next event that takes place is that one of the active coefficients becomes zero. Therefore we have to calculate $\Delta s_{j \text { new }}^{1}$ for $j \in \mathcal{A}$ as

$$
\Delta s_{\text {new }}^{1}=-\frac{a_{j}(\Delta s)}{\frac{d a_{j}}{d s}}
$$

the way we have to change $s$ such that $a_{j}(s)$ becomes zero. We define $\Delta s_{\text {new }}^{1}$ as

$$
\Delta s_{\text {new }}^{1}=\min \left\{\Delta s_{j^{\prime} \text { new }}^{1}, \Delta s_{j^{\prime \prime} \text { new }}^{1}\right\}
$$

Again, if one of the $\Delta s_{j \text { new }}^{1}$ is less than zero, we set $\Delta s_{\text {new }}^{1}=\infty$.

Since we have two coefficients that are non-zero, the derivative of a residual $r e s_{i}$ 
with $i \notin \mathcal{V}_{\mathcal{H}}$ can be computed in the following way:

$$
\frac{d r e s_{i}}{d s}=-\left(\frac{d a_{j^{\prime}}}{d s} \cdot x_{i j^{\prime}}+\frac{d a_{j^{\prime \prime}}}{d s} \cdot x_{i j^{\prime \prime}}+\frac{d b}{d s}\right) .
$$

All that is left to do can be done analogously to the first case: Calculate $\Delta s_{i \text { new }}^{2}$ as

$$
\Delta s_{\text {new }}^{2}=\frac{r e s_{i}(a(\Delta s), b(\Delta s))}{-\frac{d r e s}{d s}}
$$

and $\Delta s_{\text {new }}^{2}$ as

$$
\Delta s^{2}=\min \left\{\Delta s_{\text {new }}^{2}: i \notin \mathcal{V}\right\}
$$

and choose $\Delta s_{\text {new }}$ as

$$
\Delta s_{\text {new }}=\min \left\{\Delta s_{\text {new }}^{1}, \Delta s_{\text {new }}^{2}\right\}
$$

Again we know how an optimal trajectory looks like on the interval $[\Delta s, \Delta s+$ $\left.\Delta s_{\text {new }}\right]$.

In either case we get an optimal trajectory on the interval $\left[\Delta s, \Delta s_{n e w}\right]$, and therefore altogether we know a continuous optimal trajectory on the interval $[0, \Delta s+$ $\left.\Delta s_{n e w}\right]$. The continuity derives from the fact that $a_{j}(s)$ is linear in $s$ on $(0, \Delta s)$ as well as on $\left(\Delta s, \Delta s+\Delta s_{\text {new }}\right)$ for all $j=1 \ldots k$, likewise $b(s)$ is linear in $s$ on $(0, \Delta s)$ and on $\left(\Delta s, \Delta s+\Delta s_{\text {new }}\right)$ and furthermore it holds that

$$
a_{j}(s) \rightarrow a_{j}(\Delta s) \text { for } s \nearrow \Delta s \text { as well as for } s \searrow \Delta s
$$

for all $j=1 \ldots k$ and

$$
b(s) \rightarrow b(\Delta s) \text { for } s \nearrow \Delta s \text { as well as for } s \searrow \Delta s,
$$

which is due to our construction of $(a(s), b(s))$ (see Remark V.3.6).

If we go on like that, we can construct a continuous optimal trajectory little by little.

In the following, we want to describe one general step in our construction process. Let us assume that we know $(a(\hat{s}), b(\hat{s})) \in M_{v e r}^{\text {opt }}(\hat{s})$ for a certain $\hat{s} \geq 0$ and assume that no event has happened in $\hat{s}$. Then we also know the active set $\mathcal{A}$ and the set 
of passed facilities $\mathcal{V}_{\mathcal{H}}$ of $(a(\hat{s}), b(\hat{s}))$. We know that we can find optimal solutions $(a(s), b(s))$ of $\left(P_{v e r}(s)\right)$ for growing $s$ which have the same active set as well as the same set of passed facilities as $(a(\hat{s}), b(\hat{s}))$ until $s$ reaches the point where an event happens due to Lemma V.3.4. Since we assumed that no event has happened in $\hat{s}$, we know that as long as no event happens we can find $(a(s), b(s))$ that satisfy the following system of equations:

$$
\begin{aligned}
& \sum_{j \in \mathcal{A}} \operatorname{sgn}\left(a_{j}(s)\right) a_{j}(s)=s \text { and } \\
& \sum_{j \in \mathcal{A}} a_{j}(s) x_{i j}+b(s)=y_{i} \forall i \in \mathcal{V}_{\mathcal{H}} .
\end{aligned}
$$

Note that we can solve this system since $\left|\mathcal{V}_{\mathcal{H}}\right|=|\mathcal{A}|$ according to Lemma V.3.3. Theorem V.3.3 assures that $a_{j}(s), j \in \mathcal{A}$, and $b(s)$ are linear in $s$. We can calculate the derivatives of $a_{j}(s), j \in \mathcal{A}$ and $b(s)$ with respect to $s$ by solving

$$
\begin{aligned}
& \sum_{j \in \mathcal{A}} \operatorname{sgn}\left(a_{j}(s)\right) \frac{d a_{j}}{d s}=1 \text { and } \\
& \sum_{j \in \mathcal{A}} \frac{d a_{j}}{d s} x_{i j}+\frac{d b}{d s}=0 \forall i \in \mathcal{V}_{\mathcal{H}} .
\end{aligned}
$$

Therefore, as long as no event happens we can write $(a(s), b(s))$ as

$$
\begin{aligned}
a_{j}(s) & =\left\{\begin{array}{l}
a_{j}(\hat{s})+\frac{d a_{j}}{d s} \cdot \Delta s \text { if } j \in \mathcal{A} \\
0 \text { else }
\end{array}\right. \\
b(s) & =b(\hat{s})+\frac{d b}{d s} \cdot \Delta s .
\end{aligned}
$$

This gives us an optimal solution $(a(s), b(s))$ of $\left(P_{v e r}(s)\right)$ until an event happens. Now we have to calculate the stepsize $\Delta s$ such that the next time an event takes place is $\hat{s}+\Delta s$. One type of event that can happen is that one of the active coefficients becomes zero again, that is to say $a_{j}(s)=0$ for one $j \in \mathcal{A}$. For each $j \in \mathcal{A}$ we can calculate when this is going to happen since we know the derivatives of the $a_{j}$ : 


$$
\Delta s_{j}^{1}=-\frac{a_{j}(\hat{s})}{\frac{d a_{j}}{d s}} .
$$

If one of the $\Delta s_{j}^{1}$ is negative we set it to $\infty$ for the same reason we did that when starting with $s=0$. Therefore the next time an active coefficient leaves the active set is $\hat{s}+\Delta s^{1}$ with $\Delta s^{1}$ being

$$
\Delta s^{1}=\min \left\{\Delta s_{j}^{1}: j \in \mathcal{A}\right\} .
$$

The other type of event is that one of the hyperplanes corresponding to an existing facility subscripted by $i \notin \mathcal{V}_{\mathcal{H}}$ joins the set of passed facilities, in other words one of the residuals

$$
\operatorname{res}_{i}(a(s), b(s)):=\operatorname{res}\left((a(s), b(s)), H_{-x_{i}, y_{i}}\right)=y_{i}-\left(\sum_{j \in \mathcal{A}} a_{j}(s) x_{i j}+b(s)\right), i \notin \mathcal{V}_{\mathcal{H}}
$$

becomes zero. It is easy to check that $r s_{i}$ can be rewritten as

$$
\operatorname{res}_{i}(a(s), b(s))=\operatorname{res}_{i}(a(\hat{s}), b(\hat{s}))-\left(\sum_{j \in \mathcal{A}} \frac{d a_{j}}{d s} \cdot \Delta s \cdot x_{i j}+\frac{d b}{d s} \cdot \Delta s\right)
$$

and therefore we can calculate the derivatives of the residuals

$$
\frac{d r e s_{i}}{d s}=-\left(\sum_{j \in \mathcal{A}} \frac{d a_{j}}{d s} \cdot x_{i j}+\frac{d b}{d s}\right), i \notin \mathcal{V}_{\mathcal{H}}
$$

Now that we know these derivatives, we can easily compute $\Delta s_{i}^{2}$ for $i \notin \mathcal{V}_{\mathcal{H}}$ such that $\operatorname{res}_{i}(a(s), b(s))$ becomes zero for $\hat{s}+\Delta s_{i}^{2}$ :

$$
\Delta s_{i}^{2}=\frac{\operatorname{res}_{i}(a(\hat{s}), b(\hat{s}))}{-\frac{d r e s}{d s}}
$$

One more time we set $\Delta s_{i}^{2}=\infty$ if $\Delta s_{i}^{2}<0$ for an $i \notin \mathcal{V}_{\mathcal{H}}$. Since we want to know when the first event of this type will happen we set

$$
\Delta s^{2}=\min \left\{\Delta s_{i}^{2}: i \notin \mathcal{V}_{\mathcal{H}}\right\}
$$


Knowing $\Delta s^{1}$ and $\Delta s^{2}$, we let

$$
\Delta s=\min \left\{\Delta s^{1}, \Delta s^{2}\right\}
$$

and we know that the first event will happen at $\hat{s}+\Delta s$. Therefore we know a continuous optimal trajectory on the interval $[\hat{s}, \hat{s}+\Delta s]$. Additionally, knowing the derivatives of the $a_{j}$ and of $b$ it is easy to see that the derivative of the optimal objective function value with respect to $s$ is

$$
\frac{d f_{v e r}(a(s), b(s))}{d s}=\sum_{j \in \mathcal{A}}\left(\sum_{i \in L} \frac{d a_{j}}{d s} x_{i j}-\sum_{i \in R} \frac{d a_{j}}{d s} x_{i j}\right)+(|L|-|R|) \cdot \frac{d b}{d s} .
$$

Finally, we have to decide how to continue after an event has happened. As in the case were we started with $s=0$, we have to check all possible combinations of active sets and sets of passed facilities that can occur and choose the one that delivers the best objective function value. At $s=\hat{s}+\Delta s$ it is $\left|\mathcal{V}_{\mathcal{H}}\right|=|\mathcal{A}|+1$ no matter if $\Delta s=\Delta s^{1}$ or $\Delta s=\Delta s^{2}$. Due to Lemma V.3.3 and the one at a time condition, all possible combinations of active sets and sets of passed facilities are either of the form $\mathcal{A}^{\text {new }}=\mathcal{A}$ while $\mathcal{V}_{\mathcal{H}}^{\text {new }}=\mathcal{V}_{\mathcal{H}} \backslash\{i\}$ for an $i \in \mathcal{V}_{\mathcal{H}}$ or of the form $\mathcal{A}^{\text {new }}=\mathcal{A} \cup\{j\}$ for a $j \notin \mathcal{A}$ while $\mathcal{V}_{\mathcal{H}}^{\text {new }}=\mathcal{V}_{\mathcal{H}}$. In either case, again it holds that $\left|\mathcal{A}^{\text {new }}\right|=\left|\mathcal{V}_{\mathcal{H}}^{\text {new }}\right|$ and we can calculate the derivatives $\frac{d a_{j}}{d s}$ for $j \in \mathcal{A}^{\text {new }}$ and $\frac{d b}{d s}$ for every possible combination starting from the respective system of equations

$$
\begin{aligned}
& \sum_{j \in \mathcal{A}_{\text {new }} \operatorname{sgn}\left(a_{j}(s)\right) a_{j}(s)=s \text { and }} \\
& \sum_{j \in \mathcal{A}^{\text {new }}} a_{j}(s) x_{i j}+b(s)=y_{i} \forall i \in \mathcal{V}_{\mathcal{H}}^{\text {new }} .
\end{aligned}
$$

This enables us to calculate the respective derivative of the objective function value with respect to $s$ for each possible combination

$$
\begin{aligned}
\frac{d f_{\text {ver }}(a(s), b(s))}{d s}= & \sum_{j \in \mathcal{A}^{\text {new }}}\left(\sum_{i \in L^{\text {new }}} \frac{d a_{j}}{d s} x_{i j}-\sum_{i \in R^{\text {new }}} \frac{d a_{j}}{d s} x_{i j}\right) \\
& +\left(\left|L^{\text {new }}\right|-\left|R^{\text {new }}\right|\right) \cdot \frac{d b}{d s}
\end{aligned}
$$




\section{V.4. HOW TO CONSTRUCT A CONTINUOUS OPTIMAL}

and we choose the minimum of all these derivatives if it is negative. If the minimum is positive we can stop because the objective function value will not get any better and we have reached an optimal solution for the unrestricted problem $\left(P_{v e r}\right)$.

Knowing the combination of $\mathcal{A}^{\text {new }}$ and $\mathcal{V}_{\mathcal{H}}^{\text {new }}$ that leads to optimal solutions we can go on calculating the stepsize $\Delta s$ as we did above and so on until we reach an optimal solution for the unrestricted problem.

Remark V.4.1. We assumed that we start with $\hat{s}$ such that no event has happened in $\hat{s}$. If we start in $\hat{s}$ such that an event has happened in $\hat{s}$, we have to start with what has been the second step in our considerations above. First we have to decide which combination of an active set and a set of passing hyperplanes leads to the best objective function value and then we can go on with what has been the first step before and calculate a stepsize $\Delta s$.

As in the two-dimensional case, the continuous optimal trajectory that we constructed in this section has a nice geometrical interpretation in the dual $\mathbb{R}^{k+1}$. We already hinted at this fact in Remark V.3.6.

Remember that we are given a cell structure on this dual space induced by the hyperplanes $H_{-x_{i}, y_{i}}$ corresponding to the existing facilities $\left(x_{i}, y_{i}\right), i=1 \ldots n$, together with the hyperplanes $H_{j}=\left\{(a, b) \in \mathbb{R}^{k+1}: a_{j}=0\right\}, j=1, \ldots, k$. In Section $V .2$ we called the set of hyperplanes corresponding to existing facilities $\mathcal{H}$ and the set of hyperplanes $H_{j}$ we called $\mathcal{H}^{\prime \prime}$. Furthermore we denoted by $\mathcal{C}^{\prime}$ the cells induced by the hyperplanes in $\mathcal{H} \cup \mathcal{H}^{\prime \prime}$. Additional hyperplanes are given through the restriction on the slope, the set of these hyperplanes we called $\mathcal{H}^{\prime}$. Note that $\mathcal{H}^{\prime}$ is dependent on $s$ while $\mathcal{H}$ as well as $\mathcal{H}^{\prime \prime}$ do not change with $s$.

What we do to construct our optimal trajectory is that we start with a point $(a(0), b(0))$ that is a (lower) median of the set $\left\{y_{i}: i=1 \ldots n\right\}$. Note that $(a(0), b(0))$ is a vertex of a cell $C_{1} \in \mathcal{C}^{\prime}$ as we already pointed out at the beginning of this section as well as in Remark V.3.6. The set of feasible solutions $M_{v e r}(0)$ is just the $b$-axis of the dual space. Now we let $s$ grow. The set of feasible solutions $M_{v e r}(s)$ which is defined through the hyperplanes in $\mathcal{H}^{\prime}$ becomes what we called a kind of a tube around the $b$-axis, namely

$$
M_{v e r}(s)=\left\{(a, b) \in \mathbb{R}^{k+1}:\|a\|_{1} \leq s\right\}
$$




\section{LOCATING HYPERPLANES WITH A RESTRICTION ON THE SLOPE USING VERTICAL DISTANCE

Our optimal trajectory follows the edge of the cell $C_{1}$ on which we have chosen $(a(0), b(0))$ giving us for any $s$ the point $(a(s), b(s))$ lying on the edge of $C_{1}$ as well as on the surface of the tube defined by $M_{v e r}(s)$ (see Lemma V.3.5). The trajectory follows this edge chosen at $s=0$ until it reaches a vertex of $C_{1}$. From this vertex, it will follow the edge of a cell $C_{2} \in \mathcal{C}^{\prime}$ such that the point lying on this edge as well as on the surface of the tube defined by $M_{v e r}(s)$ gives the best objective value for $\left(P_{v e r}(s)\right)$ until it reaches a vertex of $C_{2}$ and so on. Therefore our trajectory is moving along different edges of cells $C \in \mathcal{C}^{\prime}$ while it always stays on the surface of $M_{v e r}(s)$ given through the set $\mathcal{H}^{\prime}$ with growing $s$.

Remark V.4.2. One can also construct a continuous optimal trajectory for our problem $\left(P_{v e r}\right)$ by applying the Karush-Kuhn-Tucker conditions to the optimization problem

$$
\min \left\|y-[X e]\left(\begin{array}{c}
a \\
b
\end{array}\right)\right\|_{1}+\lambda\|a\|_{1}, \quad \quad\left(P_{R L A D}(\lambda)\right)
$$

with $\lambda \geq 0$ being a tuning parameter, $y=\left(y_{1}, \ldots, y_{n}\right)^{t} \in \mathbb{R}^{n}, a=\left(a_{1}, \ldots, a_{k}\right)^{t} \in$ $\mathbb{R}^{k}, b \in \mathbb{R}$ and $[X e] \in \mathbb{R}^{n \times(k+1)}$ being the matrix

$$
[X e]=\left(\begin{array}{llll}
x_{11} & \ldots & x_{1 k} & 1 \\
x_{21} & \ldots & x_{2 k} & 1 \\
\vdots & \ddots & \vdots & \vdots \\
x_{n 1} & \ldots & x_{n k} & 1
\end{array}\right)
$$

Basically, this is what is done in [WGZ06]. Here it is important to note that we have shown the equivalence of the above optimization problem $\left(P_{R L A D}(\lambda)\right)$ and the problem $\left(P_{\text {ver }}(s)\right)$ in Chapter [II.

Since we are more interested in the geometrical nature of the trajectory we have chosen the approach of capitalizing on the geometrical properties of the cell structure in the dual space.

Remark V.4.3. We already mentioned the equivalence of the two optimization problems $\left(P_{v e r}(s)\right)$ and $\left(P_{R L A D}(\lambda)\right)$ in the previous remark. Due to this equivalence, the optimal trajectory we constructed in this section is an optimal solution path for the RLAD regression as well, as we already pronounced at the end of Chapter II. 
We want to present an easy example to demonstrate the construction of a continuous optimal trajectory that we described in this section. We use the setting that we already used in Example V.1.1, that is we have again $k=2$ and $n=4$.

Example V.4.1. Let the set of existing facilities $\mathcal{E}=\left\{\left(x_{1}, y_{1}\right),\left(x_{2}, y_{2}\right),\left(x_{3}, y_{3}\right)\right.$, $\left.\left(x_{4}, y_{4}\right)\right\} \subseteq \mathbb{R}^{3}$ be as in ExampleV.1.1. We want to construct the complete optimal trajectory on the interval $\left[0, \frac{215}{94}\right]$ since we know that $\left(\frac{51}{94}, \frac{82}{47}, \frac{230}{47}\right)$ is an optimal solution to $\left(P_{\text {ver }}\right)$ and therefore $s^{*}=\|a\|_{1}=\frac{215}{94}$.

We start the solution path in $s=0$ with $(0,0, b(0)), b(0)$ being the lower median of the set $\{1,-1,-3,4\}$. Therefore we start with $(a(0), b(0))=(0,0,-1)$, the value of the objective function being $f_{v e r}(a(0), b(0))=9$. As the intersection of $H_{-x_{2}, y_{2}}$, $H_{1}$ and $H_{2}$, the point $(a(0), b(0))=(0,0,-1)$ is a vertex of a cell in $\mathcal{C}^{\prime}$.

Now we have to check which one of the coefficients will join the active set $\mathcal{A}$ first. We know that the set of passed facilities is $\mathcal{V}_{\mathcal{H}}=\{2\}$ and this gives us $L=\{3\}$ and $R=\{1,4\}$. We choose the coefficient as

$$
\underset{j}{\arg \min }\left\{\frac{d f_{v e r}\left(a_{j}(s), b(s)\right)}{d s}\right\},
$$

therefore we calculate $\frac{d f_{v e r}\left(a_{j}(s), b(s)\right)}{d s}$ for $j=1,2$ regarding that $\mathcal{V}_{\mathcal{H}}=\{2\}$. We get

$$
\frac{d f_{v e r}\left(a_{1}(s), b(s)\right)}{d s}=-1-(-12+8)+2=5
$$

and

$$
\frac{d f_{v e r}\left(a_{2}(s), b(s)\right)}{d s}=-2-\left(\frac{3}{2}-3\right)-4=-\frac{9}{2},
$$

so $j=1$ is the first coefficient joining the active set. The sign of $a_{1}(s)$ will be $\operatorname{sgn}\left(a_{1}(s)\right)=-1$.

Now we have $\mathcal{A}=\{1\}$ and $\mathcal{V}_{\mathcal{H}}=\{2\}$. We know that $\left(a_{1}(s), 0, b(s)\right)$ has to satisfy the following system of equations

$$
\begin{aligned}
-a_{1}(s) & =s \\
2 a_{1}(s)+b(s) & =-1
\end{aligned}
$$


as long as no event happens. This enables us to calculate the derivatives of $a_{1}(s)$ and $b(s)$ with respect to $s$ by solving

$$
\begin{aligned}
\frac{d a_{1}}{d s} & =-1 \\
2 \frac{d a_{1}}{d s}+\frac{d b}{d s} & =0 .
\end{aligned}
$$

We get that $\frac{d a_{1}}{d s}=-1$ and $\frac{d b}{d s}=2$. Note that $a_{1}(s)$ will never become zero with growing $s$ which is why we set $\Delta s^{1}=\infty$.

Now we can calculate the derivatives of the residuals for all $i \notin \mathcal{V}_{\mathcal{H}}$ :

$$
\begin{aligned}
& \frac{d r e s_{1}}{d s}=-(12+2)=-14 \\
& \frac{d r e s_{3}}{d s}=-(1+2)=-1 \\
& \frac{d r e s_{4}}{d s}=-(-8+2)=6 .
\end{aligned}
$$

The stepsizes $\Delta s_{i}^{2}$ for $i \notin \mathcal{V}$ can be calculated using the residuals of the respective hyperplanes at $s=0$.

$$
\begin{aligned}
& \Delta s_{1}^{2}=\frac{2}{14}=\frac{1}{7} \\
& \Delta s_{3}^{2}=\frac{-2}{3}=-\frac{2}{3} \\
& \Delta s_{4}^{2}=\frac{5}{-6}=-\frac{5}{6}
\end{aligned}
$$

therefore we get $\Delta s_{1}^{2}=\frac{1}{7}$ while $\Delta s_{3}^{2}=\Delta s_{4}^{2}=\infty$. This gives us $\Delta s^{2}=\frac{1}{7}$.

Finally we have to choose $\Delta s$ as

$$
\min \left\{\Delta s^{1}, \Delta s^{2}\right\}
$$

therefore $\Delta s=\frac{1}{7}$ and we know that the first event will take place at $s=\frac{1}{7}$. Note that we in fact know what kind of event that will be, namely $i=1$ will join the set of passed facilities $\mathcal{V}_{\mathcal{H}}$.

For $s=\frac{1}{7}$ we therefore get $\left(a_{1}\left(\frac{1}{7}\right), a_{2}\left(\frac{1}{7}\right), b\left(\frac{1}{7}\right)\right)=\left(-\frac{1}{7}, 0,-\frac{5}{7}\right)$ as an optimal solution with active set $\mathcal{A}=\{1\}$ and set of passed facilities $\mathcal{V}_{\mathcal{H}}=\{1,2\}$. Note 


\section{V.4. HOW TO CONSTRUCT A CONTINUOUS OPTIMAL}

that $\left(a_{1}\left(\frac{1}{7}\right), a_{2}\left(\frac{1}{7}\right), b\left(\frac{1}{7}\right)\right)$ is the intersection of $H_{-x_{1}, y_{i}}, H_{-x_{2}, y_{2}}$ and $H_{2}$, hence it is a vertex of a cell in $\mathcal{C}^{\prime}$. The value of the objective function is $f_{\text {ver }}\left(-\frac{1}{7}, 0,-\frac{5}{7}\right)=$ $9-\frac{1}{7} \cdot 5=\frac{58}{7}$. Moreover we know that $\left(a_{1}(s), a_{2}(s), b(s)\right)$ with

$$
\begin{aligned}
a_{1}(s) & =-1 \cdot s \\
a_{2}(s) & =0 \\
b(s) & =-1+2 \cdot s
\end{aligned}
$$

gives us an optimal solution to $\left(P_{\text {ver }}(s)\right)$ for any $s \in\left[0, \frac{1}{7}\right]$.

Now that we have $|\mathcal{A}|=1$ while $\left|\mathcal{V}_{\mathcal{H}}\right|=2$ we have several options of what should happen next. Either $j=2$ might join the active set or one of the two elements in $\mathcal{V}_{\mathcal{H}}$ might leave the set of passed facilities. We have to check which of these options is the best one in terms of the derivative of $f_{\text {ver }}$ with respect to $s$.

Let us start with the different possible cases of one hyperplane leaving $\mathcal{V}_{\mathcal{H}}$.

- $\mathcal{A}=\{1\}, \mathcal{V}_{\mathcal{H}}=\{2\}$

Note that now it is $R=\{4\}$ and $L=\{1,3\}$. Since the derivatives of $a_{1}(s)$ and $b(s)$ are the same as above we get

$$
\frac{d f_{\text {ver }}}{d s}=-((-12-1)-8)+2=23
$$

- $\mathcal{A}=\{1\}, \mathcal{V}_{\mathcal{H}}=\{1\}$

First we have to calculate the derivatives of $a_{1}(s)$ and $b(s)$ by solving

$$
\begin{aligned}
\frac{d a_{1}}{d s} & =-1 \\
-12 \frac{d a_{1}}{d s}+\frac{d b}{d s} & =0
\end{aligned}
$$

and we get $\frac{d a_{1}}{d s}=-1$ and $\frac{d b}{d s}=-12$. Since it is $L=\{3\}$ and $R=\{2,4\}$ in this case, we get

$$
\frac{d f_{\text {ver }}}{d s}=-(-1-8+12)+12=9 \text {. }
$$


What is left to check is the case with $j=2$ joining the active set, distinguishing the cases $a_{2}(s)>0$ and $a_{2}(s)<0$.

- $\mathcal{A}=\{1,2\}, \mathcal{V}_{\mathcal{H}}=\{1,2\}$

Again we first have to calculate the derivatives of $a_{1}(s), a_{2}(s)$ and $b(s)$. Solving

$$
\begin{array}{r}
-\frac{d a_{1}}{d s}+\operatorname{sgn}\left(a_{2}(s)\right) \frac{d a_{2}}{d s}=1 \\
-12 \frac{d a_{1}}{d s}+\frac{3}{2} \frac{d a_{2}}{d s}+\frac{d b}{d s}=0 \\
2 \frac{d a_{1}}{d s}-4 \frac{d a_{2}}{d s}+\frac{d b}{d s}=0
\end{array}
$$

we get $\frac{d a_{1}}{d s}=\frac{11}{17}, \frac{d a_{2}}{d s}=\frac{28}{17}$ and $\frac{d b}{d s}=\frac{90}{17}$ if $\operatorname{sgn}\left(a_{2}(s)\right)=+1$ while we get $\frac{d a_{1}}{d s}=-\frac{11}{39}$, $\frac{d a_{2}}{d s}=-\frac{28}{39}$ and $\frac{d b}{d s}=-\frac{30}{13}$ if $\operatorname{sgn}\left(a_{2}(s)\right)=-1$. Since we have $L=\{3\}$ and $R=\{4\}$ in both cases, we can calculate the derivatives of $f_{\text {ver }}$ as

$$
\frac{d f_{v e r}}{d s}=\frac{11}{17} \cdot(-9)+\frac{28}{17} \cdot 1=-\frac{71}{17}
$$

if $\operatorname{sgn}\left(a_{2}(s)\right)=+1$ and

$$
\frac{d f_{v e r}}{d s}=-\frac{11}{39} \cdot(-9)-\frac{28}{39} \cdot 1=\frac{71}{39}
$$

if $\operatorname{sgn}\left(a_{2}(s)\right)=-1$.

Looking at the derivatives of $f_{\text {ver }}$ of all different cases shows that the one with $j=2$ joining the active set with positive sign is the only case with negative derivative. Therefore this case yields the best objective function values and our new active set becomes $\mathcal{A}=\{1,2\}$ with positive $a_{2}(s)$ while our new set of passed facilities is $\mathcal{V}_{\mathcal{H}}=\{1,2\}$.

Since we already know the derivatives of $a_{1}(s)$ and $a_{2}(s)$ we can calculate the stepsize

$$
\Delta s_{1}^{1}=-\frac{-\frac{1}{7}}{\frac{11}{17}}=\frac{17}{77}
$$

while it is

$$
\Delta s_{2}^{1}=\infty
$$


Altogether we get $\Delta s^{1}=\frac{17}{77}$.

Now we have to calculate the stepsizes $\Delta s_{3}^{2}$ and $\Delta s_{4}^{2}$. In order to do so we need the residuals at $s=\frac{1}{7}$ :

$$
\begin{aligned}
& \operatorname{res}_{3}\left(a\left(\frac{1}{7}\right), b\left(\frac{1}{7}\right)\right)=-3-\left(\frac{1}{7}-\frac{5}{7}\right)=-\frac{17}{7} \\
& \operatorname{res}_{4}\left(a\left(\frac{1}{7}\right), b\left(\frac{1}{7}\right)\right)=4-\left(-\frac{1}{7} \cdot 8-\frac{5}{7}\right)=\frac{41}{7} .
\end{aligned}
$$

We get the stepsizes by calculating

$$
\begin{aligned}
& \Delta s_{3}^{2}=\frac{-\frac{17}{7}}{\frac{11}{17} \cdot(-1)+\frac{28}{17} \cdot(-2)+\frac{90}{17}}=-\frac{289}{161} \text { and } \\
& \Delta s_{4}^{2}=\frac{\frac{41}{7}}{\frac{11}{17} \cdot 8+\frac{28}{17} \cdot(-3)+\frac{90}{17}}=\frac{697}{658} .
\end{aligned}
$$

Since $\Delta s_{3}^{2}$ is negative we have to set $\Delta s_{3}^{2}=\infty$ and get $\Delta s^{2}=\frac{697}{658}$. We choose

$$
\Delta s=\min \left\{\frac{17}{77}, \frac{697}{658}\right\}=\frac{17}{77}
$$

and know how the trajectory looks on the interval $\left[\frac{1}{7}, \frac{4}{11}\right]$, namely

$$
\begin{aligned}
& a_{1}(s)=-\frac{1}{7}+\frac{11}{17} \cdot\left(s-\frac{1}{7}\right), \\
& a_{2}(s)=\frac{28}{17} \cdot\left(s-\frac{1}{7}\right) \text { and } \\
& b(s)=-\frac{5}{7}+\frac{90}{17} \cdot\left(s-\frac{1}{7}\right)
\end{aligned}
$$

for $s \in\left[\frac{1}{7}, \frac{4}{11}\right]$.

For $s=\frac{4}{11}$ we get the optimal solution $\left(a\left(\frac{4}{11}\right), b\left(\frac{4}{11}\right)\right)=\left(0, \frac{4}{11}, \frac{5}{11}\right)$ with objective function value $f_{\text {ver }}\left(a\left(\frac{4}{11}\right), b\left(\frac{4}{11}\right)\right)=\frac{81}{11}$. The active set now is $\mathcal{A}=\{2\}$ while the set of passing hyperplanes is $\mathcal{V}_{\mathcal{H}}=\{1,2\}$. Again $\left(a\left(\frac{4}{11}\right), b\left(\frac{4}{11}\right)\right)$ is a vertex of a cell in $\mathcal{C}^{\prime}$ as the intersection of $H_{-x_{1}, y_{i}}, H_{-x_{2}, y_{2}}$ and $H_{1}$.

At this point again we have to check which possible options our trajectory has to continue and which one of these gives the best objective function value. We start with the different possible cases of one hyperplane leaving $\mathcal{V}_{\mathcal{H}}$. 
- $\mathcal{A}=\{2\}, \mathcal{V}_{\mathcal{H}}=\{1\}$

In this case we have $L=\{3\}$ while it is $R=\{2,4\}$.

We can calculate the derivatives of $a_{2}(s)$ and $b(s)$ with respect to $s$ by solving

$$
\begin{aligned}
\frac{d a_{2}}{d s} & =1 \\
\frac{3}{2} \cdot \frac{d a_{2}}{d s}+\frac{d b}{d s} & =0
\end{aligned}
$$

and get $\frac{d a_{2}}{d s}=1$ and $\frac{d b}{d s}=-\frac{3}{2}$. Therefore it is

$$
\frac{d f_{v e r}}{d s}=-2-(-4-3)+\frac{3}{2}=\frac{13}{2}
$$

- $\mathcal{A}=\{2\}, \mathcal{V}_{\mathcal{H}}=\{2\}$

The derivatives of $a_{2}(s)$ and $b(s)$ derived from

$$
\begin{aligned}
\frac{d a_{2}}{d s} & =1 \\
-4 \cdot \frac{d a_{2}}{d s}+\frac{d b}{d s} & =0
\end{aligned}
$$

are $\frac{d a_{2}}{d s}=1$ and $\frac{d b}{d s}=4$. Since $L=\{1,3\}$ and $R=\{4\}$ we get

$$
\frac{d f_{v e r}}{d s}=\left(\frac{3}{2}-2+3\right)+4=\frac{13}{2}
$$

again.

The other case we have to check is $j=1$ joining the active set again but this time having positive sign. Note that $j=1$ joining the active set again with negative sign does not make any sense since otherwise $\operatorname{sgn}\left(a_{1}(s)\right) \cdot a_{1}(s)+\operatorname{sgn}\left(a_{2}(s)\right) \cdot a_{2}(s)=s$ does not hold any more. 
- $\mathcal{A}=\{1,2\}, \mathcal{V}_{\mathcal{H}}=\{1,2\}, \operatorname{sgn}\left(a_{1}(s)\right)=+1$

Solving

$$
\begin{aligned}
\frac{d a_{1}}{d s}+\frac{d a_{2}}{d s} & =1 \\
-12 \frac{d a_{1}}{d s}+\frac{3}{2} \frac{d a_{2}}{d s}+\frac{d b}{d s} & =0 \\
2 \frac{d a_{1}}{d s}-4 \frac{d a_{2}}{d s}+\frac{d b}{d s} & =0
\end{aligned}
$$

gives us $\frac{d a_{1}}{d s}=\frac{11}{39}, \frac{d a_{2}}{d s}=\frac{28}{39}$ and $\frac{d b}{d s}=\frac{30}{13}$. We have $L=\{3\}$ and $R=\{4\}$ and therefore

$$
\frac{d f_{\text {ver }}}{d s}=\frac{11}{39} \cdot(-1-8)+\frac{28}{39} \cdot(-2+3)=-\frac{71}{39} .
$$

Therefore we choose $\mathcal{A}=\{1,2\}$ with $a_{1}(s)$ and $a_{2}(s)$ being positive as our new active set and our new set of passed facilities is $\mathcal{V}_{\mathcal{H}}=\{1,2\}$. Since $a_{1}(s)$ and $a_{2}(s)$ as well as the derivatives of $a_{1}(s)$ and $a_{2}(s)$ are all positive in this case we get the stepsizes

$$
\Delta s_{1}^{1}=\infty
$$

and

$$
\Delta s_{2}^{1}=\infty
$$

therefore it is $\Delta s^{1}=\infty$.

We still have to calculate the stepsizes $\Delta_{i}^{2}$ for $i \notin \mathcal{V}$ :

$$
\begin{aligned}
& \Delta s_{3}^{2}=\frac{-\frac{30}{11}}{-\frac{11}{39}+\frac{28}{39} \cdot(-2)+\frac{30}{13}}=-\frac{1170}{253} \\
& \Delta s_{4}^{2}=\frac{\frac{51}{11}}{\frac{11}{39} \cdot 8+\frac{28}{39} \cdot(-3)+\frac{30}{13}}=\frac{1989}{1034}
\end{aligned}
$$

where

$$
\operatorname{res}_{3}\left(a\left(\frac{4}{11}\right), b\left(\frac{4}{11}\right)\right)=-\frac{30}{11}
$$

and

$$
\operatorname{res}_{4}\left(a\left(\frac{4}{11}\right), b\left(\frac{4}{11}\right)\right)=\frac{51}{11} .
$$

Therefore we get $\Delta s_{3}^{2}=\infty$ and so it is $\Delta s=\Delta s_{4}^{2}=\frac{1989}{1034}$. This gives us the optimal 


\section{LOCATING HYPERPLANES WITH A RESTRICTION ON THE SLOPE USING VERTICAL DISTANCE

trajectory on the interval $\left[\frac{4}{11}, \frac{215}{94}\right]$ through

$$
\begin{aligned}
a_{1}(s) & =\frac{11}{39} \cdot\left(s-\frac{4}{11}\right) \\
a_{2}(s) & =\frac{4}{11}+\frac{28}{39} \cdot\left(s-\frac{4}{11}\right) \text { and } \\
b(s) & =\frac{5}{11}+\frac{30}{13} \cdot\left(s-\frac{4}{11}\right)
\end{aligned}
$$

for $s \in\left[\frac{4}{11}, \frac{215}{94}\right]$. Note that for $s=\frac{215}{94}$ we get $\left(a\left(\frac{215}{94}\right), b\left(\frac{215}{94}\right)\right)=\left(\frac{51}{94}, \frac{42}{47}, \frac{230}{47}\right)$ which is indeed an optimal solution to $\left(P_{\text {ver }}\right)$, see Example V.1.1. Moreover it is a vertex of a cell in $\mathcal{C}^{\prime}$ as the intersection of $H_{-x_{1}, y_{1}}, H_{-x_{2}, y_{2}}$ and $H_{-x_{4}, y_{4}}$. The new active set is $\mathcal{A}=\{1,2\}$, the set of passed facilities is $\mathcal{V}_{\mathcal{H}}=\{1,2,4\}$ and the value of the objective function is $f_{\text {ver }}\left(a\left(\frac{215}{94}\right), b\left(\frac{215}{94}\right)\right)=\frac{363}{94}$.

Nevertheless we want to check whether our construction method would stop in the optimal solution, even if we do not want to do it in every detail. It is easy to see that the only event that could actually happen is $i=2$ leaving the set of passed facilities. If any other hyperplane leaves $\mathcal{V}_{\mathcal{H}}$ the solution would no longer be pseudo-halving and therefore cannot be optimal. On the other hand, the residual of $i=3$ will never become zero since $\Delta s_{3}^{2}=\infty$. No coefficient will leave the active set since $a_{1}(s)$ and $a_{2}(s)$ are both positive and so are their derivatives.

Let therefore $\mathcal{A}=\{1,2\}$ and $\mathcal{V}_{\mathcal{H}}=\{1,4\}$. Now we can calculate the derivative of the objective function value with respect to $\mathrm{s}$. We see that

$$
\frac{d f_{v e r}}{d s}=\frac{53}{49}>0
$$

Therefore this case would not lead to a better value of the objective function and we can stop at $s=\frac{215}{94}$.

Remember that in the case of line location with vertical distances we had the nice result that the sign of the slope of any line optimal to $\left(P_{v e r}(s)\right), s \geq 0$, is the same as the sign of the slope of an optimal solution to the unrestricted problem $\left(P_{v e r}\right)$ (see Theorem I.2.4). This means that the sign of the slope of a line optimal to a restricted problem is always the same as the sign of the slope of a line optimal to the unrestricted problem no matter how $s$ is chosen since all optimal lines to the unrestricted problem have the same sign in slope (see Theorem I.2.3). 


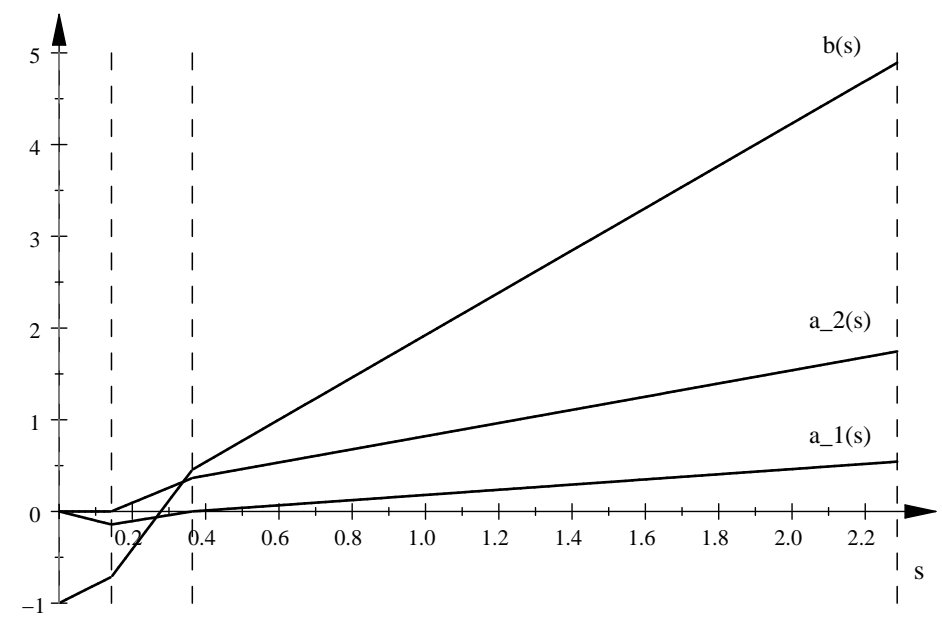

Figure V.4.1. The coefficients of the solutions on the optimal trajectory constructed in Example V.4.1 with growing $s$. The dashed vertical lines indicate were events happen.

It would be good to have an equivalent result for the case of hyperplane location as well. A generalization of the result in the case of line location could be the following statement: If $\left(a^{*}, b^{*}\right)$ is the optimal solution for the unrestricted problem $\left(P_{v e r}\right)$ lying on the optimal trajectory following the (lower) median path that we can construct it holds that for any $s \geq 0$ and the appropriate optimal solution $(a(s), b(s))$ of the restricted problem $\left(P_{\text {ver }}(s)\right)$ lying on the same optimal trajectory $\operatorname{sgn}\left(a(s)_{j}\right)=\operatorname{sgn}\left(a_{j}^{*}\right)$ is true for all $j \in\left\{j: a_{j}^{*} \neq 0\right.$ and $\left.a(s)_{j} \neq 0\right\}$. Unfortunately this is not true in general as Example V.4.1 shows. The optimal solution for the unrestricted problem $\left(P_{v e r}\right)$ that is lying on the optimal trajectory is $\left(a_{1}^{*}, a_{2}^{*}, b^{*}\right)=$ $\left(\frac{51}{94}, \frac{42}{47}, \frac{230}{47}\right)$, therefore $\operatorname{sgn}\left(a_{1}^{*}\right)=1$. If we choose $s \in\left(0, \frac{4}{11}\right)$ the sign of $a_{1}(s)$ is $\operatorname{sgn}\left(a_{1}(s)\right)=-1$ and therefore obviously $\operatorname{sgn}\left(a_{1}(s)\right) \neq \operatorname{sgn}\left(a_{1}^{*}\right)$. Figure V.4.1 illustrates that $a_{1}(s)$ changes its sign in $s=\frac{4}{11}$.

But it is possible to show a weaker result by taking advantage of the fact that we can construct a continuous optimal trajectory.

Lemma V.4.1. Let

$$
\begin{aligned}
t: \mathbb{R}_{0}^{+} & \rightarrow \mathbb{R}^{k+1} \\
s & \mapsto(a(s), b(s))
\end{aligned}
$$


be a continuous optimal trajectory of our problem $\left(P_{\text {ver }}(s)\right)$ and let $\left(a^{*}, b^{*}\right) \in M_{v e r}^{\text {opt }}$ be the optimal solution to $\left(P_{\text {ver }}\right)$ lying on $t$. There exists a $s^{\prime}$ such that $0 \leq s^{\prime}<$ $\left\|a^{*}\right\|_{1}$ and for all $s>s^{\prime}$ it holds that

$$
\operatorname{sgn}\left(a(s)_{j}\right)=\operatorname{sgn}\left(a_{j}^{*}\right) \text { for all } j \in\left\{j: a_{j}^{*} \neq 0 \text { and } a(s)_{j} \neq 0\right\}
$$

where $(a(s), b(s)) \in M_{v e r}^{\text {opt }}(s)$ is the optimal solution to $\left(P_{\text {ver }}(s)\right)$ lying on the trajectory $t$.

Proof. We know that $t$ is continuous in $s$ and therefore it is in particular continuous in $s^{*}:=\left\|a^{*}\right\|_{1}$. If we choose $\epsilon>0$ small enough then

$$
\operatorname{sgn}\left(a(s)_{j}\right)=\operatorname{sgn}\left(a_{j}^{*}\right) \text { for all } j \in\left\{j: a_{j}^{*} \neq 0 \text { and } a(s)_{j} \neq 0\right\}
$$

for all $s \in\left(s^{*}-\epsilon, s^{*}\right)$ when $(a(s), b(s))$ is the appropriate optimal solution to $\left(P_{v e r}(s)\right)$ lying on the trajectory $t$. This is due to the continuity of $t$ in $s^{*}$.

But then we can choose $s^{\prime}$ as $s^{\prime}=s^{*}-\hat{\epsilon}$ with $\hat{\epsilon}$ being the supremum of all $\epsilon$ that are small enough to fulfil the condition above and we are done. 



\section{Chapter VI}

\section{Extensions}

In this chapter we want to extend some of the results of the previous chapters to a more general frame. In the first section we look at the location of a line in the normed plane. Afterwards we discuss whether it is possible to preserve some of the results presented in Chapter $\mathrm{V}$ for hyperplane location problems using horizontal distance and rectangular distance as it was the case for the location of a line.

\section{VI.1 Locating lines in the normed plane with a restriction on the slope}

In Chapter [1 and Chapter [II we examined the location of a line with a restriction on the slope using the vertical distance and the horizontal distance, respectively. In this section, we deal with the problem of locating a line with a restriction on the slope when the distance measure $d$ is derived from an arbitrary norm. We will show that there is a strong connection to the vertical case as well as to the horizontal case and that most of the results derived in Chapter \ and Chapter 【II still hold if the distance measure is derived from a norm. In order to do so, the results concerning the unrestricted location of a line using a distance measure derived from a norm presented in [Sch99] will be helpful. Due to the connection to the vertical distance and the horizontal distance, the extension to distance measures derived from a norm needs not to be discussed in all detail.

We start with the geometric definition of a norm. 
Definition VI.1.1. ([Min67]) Let B be a compact convex set in $\mathbb{R}^{n}$ with nonempty interior which is symmetric with respect to the origin. Let $x \in \mathbb{R}^{n}$. Then define the norm $\gamma: \mathbb{R}^{n} \rightarrow \mathbb{R}$ as

$$
\gamma(x):=\inf \{\lambda>0: x \in \lambda B\}
$$

It is well known that $\gamma$ satisfies the properties

$$
\begin{aligned}
\gamma(x) & \geq 0 \\
\gamma(x) & =0 \Leftrightarrow x=0 \\
\gamma(\lambda x) & =|\lambda| \gamma(x) \text { and } \\
\gamma(x+y) & \leq \gamma(x)+\gamma(y) .
\end{aligned}
$$

for all $x, y \in \mathbb{R}^{n}$ and $\lambda \in \mathbb{R}$, see the following lemma. This lemma also ensures that all norms can be characterized by their unit balls $B$.

Lemma VI.1.1. ([Min67]) The following hold:

1. Let $\gamma$ be given as in Definition [VI.1.1. Then $\gamma$ satisfies (1) - (4).

2. Let $\gamma: \mathbb{R}^{n} \rightarrow \mathbb{R}_{\geq 0}$ be given such that $\gamma$ satisfies $(1)$ - (4). Then its unit ball

$$
B_{\gamma}=\left\{x \in \mathbb{B}^{n}: \gamma(x) \leq 1\right\}
$$

is a compact convex set with nonempty interior which is symmetric with respect to the origin.

Now we want to formulate our problem with a distance measure derived from a norm $\gamma$. Let $\mathcal{E}=\left\{\left(x_{1}, y_{1}\right), \ldots,\left(x_{n}, y_{n}\right)\right\} \subseteq \mathbb{R}^{2}$ be the set of existing facilities and let $d$ be a distance measure derived from a norm with unit ball $B$. Our aim is to locate a non-vertical line $l_{a, b}$ such that the sum of distances to the existing facilities is minimized. We will only investigate non-vertical lines in this section. This is in line with our approach in Chapter III and again this is not a restriction. In order to determine the distance between a point $(x, y)$ and a line $l_{a, b}$ we can dilate the unit ball around $(x, y)$ until it touches the line. 
Lemma VI.1.2. ([Sch99]) Let d be a distance derived from a norm with unit ball $B$. Then for any line $l_{a, b}$ and any point $(x, y)$ in the plane

$$
d\left((x, y), l_{a, b}\right)=\min \left\{|\lambda|:((x, y)+\lambda B) \cap l_{a, b} \neq \emptyset\right\} .
$$

Now that we know how the distance between a point and a line looks like we can formulate our problem as

$$
\begin{aligned}
& \min f_{d}(a, b)=\min \sum_{i=1}^{n} d\left(\left(x_{i}, y_{i}\right), l_{a, b}\right) \\
& \text { s.t. }|a| \leq s
\end{aligned}
$$

with $s \geq 0$.

In order to verify that most of the results derived in Chapter [and Chapter III hold for a metric derived from a norm as well, we first have to define another location problem. As it is done in [Sch99] for the unrestricted line location problem, we first introduce the $t$-distance. Afterwards we consider the problem of locating a line with a restriction on the slope using the $t$-distance which will lead to the connection between a metric derived by a norm and the vertical distance.

We prefer a geometrical approach here. An alternative - and more analytical approach would be to use the formula for distances derived by norms between a point and a hyperplane presented in [PC01].

Definition VI.1.2. ([Sch99]) Let $t \in \mathbb{R}^{2}$ be a given direction. For two points $x$ and $y$ in the plane define the $t$-distance $d_{t}(x, y)$ by

$$
d_{t}(x, y):=\gamma_{t}(y-x)
$$

where

$$
\gamma_{t}(x):=\left\{\begin{array}{l}
|\alpha| \text { if } x=\alpha t \\
\infty \text { else }
\end{array}\right.
$$

The $t$-distance between a point $(x, y)$ and a line $l_{a, b}$ can be determined as in the following lemma. 
Lemma VI.1.3. ([Sch99]) For $t \in \mathbb{R}^{2},(x, y) \in \mathbb{R}^{2}$ and any line $l_{a, b} \subseteq \mathbb{R}^{2}$

$$
d_{t}\left((x, y), l_{a, b}\right):=\min \left\{|\lambda|:(x, y)+\lambda t \in l_{a, b}\right\}
$$

where $\min \emptyset:=\infty$.

Note that the $t$-distance is a generalisation of $d_{v e r}$ and $d_{h o r}$ since the length of the horizontal line segment from $(x, y)$ to $l_{a, b}$ is given by $d_{e_{1}}\left((x, y), l_{a, b}\right)=$ $d_{\text {hor }}\left((x, y), l_{a, b}\right)$ while the length of the vertical line segment is given by $d_{e_{2}}\left((x, y), l_{a, b}\right)=d_{v e r}\left((x, y), l_{a, b}\right)$, where $e_{1}$ and $e_{2}$ are the unit vectors of $\mathbb{R}^{2}$.

We can now look at the optimization problem

$$
\begin{aligned}
& \min f_{d_{t}}(a, b)=\min \sum_{\left(x_{i}, y_{i}\right) \in \mathcal{E}} d_{t}\left(\left(x_{i}, y_{i}\right), l_{a, b}\right) \quad\left(P_{d_{t}}(s)\right) \\
& \text { s.t. }|a| \leq s
\end{aligned}
$$

The next lemma shows how to draw a connection between a problem of the type $\left(P_{d_{t}}(s)\right)$ and a vertical problem.

Lemma VI.1.4. ([Sch99]) Let $p, q \in \mathbb{R}^{2}$ and $D$ be a linear transformation with

$$
\begin{aligned}
& \text { 1. } D(p)=q \\
& \text { 2. } \operatorname{det}(D) \neq 0 \text {. }
\end{aligned}
$$

Then for all $(x, y) \in \mathbb{R}^{2}$

$$
d_{q}\left(D(x, y), D\left(l_{a, b}\right)\right)=d_{p}\left((x, y), l_{a, b}\right)
$$

where $D\left(l_{a, b}\right):=\left\{D(x, y):(x, y) \in l_{a, b}\right\}$.

Such a transformation $D$ exists whenever $p \neq 0$ and $q \neq 0$. Using Lemma VI.1.4 with $p=t$ and $q=e_{2}$ we get

$$
d_{e_{2}}\left(D(x, y), D\left(l_{a, b}\right)\right)=d_{t}\left((x, y), l_{a, b}\right)
$$


for all $(x, y) \in \mathbb{R}^{2}$. For our objective function this means that

$$
\min \sum_{\left(x_{i}, y_{i}\right) \in \mathcal{E}} d_{t}\left(\left(x_{i}, y_{i}\right), l_{a, b}\right)=\min \sum_{\left(x_{i}, y_{i}\right) \in \mathcal{E}} d_{v e r}\left(D\left(x_{i}, y_{i}\right), D\left(l_{a, b}\right)\right) .
$$

Now we have to check what happens to our set of feasible solutions $M_{d_{t}}=$ $\{(a, b):|a| \leq s\}$. We only want to investigate lines which have a slope that is different from the "slope of $t$ " which is defined as $s_{t}=\frac{t_{2}}{t_{1}}$ since a line with slope $s_{t}$ can only be optimal to $\left(P_{d_{t}}(s)\right)$ if all existing facilities lie on that line. This is a trivial case and can be neglected.

Two cases can occur depending on the transformation $D$. Denote by $(\tilde{x}, \tilde{y})$ and $l_{\tilde{a}, \tilde{b}}$ a transformed point $D(x, y)$ and a transformed line $l_{a, b}$, respectively.

One type of feasible set for the vertical problem in the transformed system is of the form

$$
\tilde{M}_{d_{v e r}}^{1}=\left\{(\tilde{a}, \tilde{b}): \tilde{s}_{1} \leq \tilde{a} \leq \tilde{s}_{2}\right\}
$$

with $\tilde{s}_{1}<0$ and $\tilde{s}_{2}>0$. Here, $\tilde{s}_{1}$ is connected to $-s$ and $\tilde{s}_{2}$ is connected to $s$ in the untransformed system in the way that it is $a=-s$ if $\tilde{a}=\tilde{s}_{1}$ and $a=s$ if $\tilde{a}=\tilde{s}_{2}$.

The second type of feasible set that can occur is of the form

$$
\tilde{M}_{d_{v e r}}^{2}=\left\{(\tilde{a}, \tilde{b}): \tilde{a} \leq \tilde{s}_{1} \vee \tilde{a} \geq \tilde{s}_{2}\right\}
$$

again it is $\tilde{s}_{1}<0$ and $\tilde{s}_{2}>0$. This time, $\tilde{s}_{1}$ is connected to $s$ and $\tilde{s}_{2}$ is connected to $-s$ in the untransformed system in the same sense as in the first case.

Altogether we know that the problem $\left(P_{d_{t}}(s)\right)$ is equivalent to a problem $\left(\tilde{P}_{v e r}(\tilde{s})\right)$ with vertical distance of the form

$$
\begin{aligned}
& \min \sum_{i=1}^{n} d_{v e r}\left(\left(\tilde{x}_{i}, \tilde{y}_{i}\right), l_{\tilde{a}, \tilde{b}}\right) \\
& \text { s.t. }(\tilde{a}, \tilde{b}) \in \tilde{M}_{d_{v e r}}
\end{aligned}
$$

with $\tilde{M}_{d_{v e r}} \in\left\{\tilde{M}_{d_{v e r}}^{1}, \tilde{M}_{d_{v e r}}^{2}\right\}$.

From now on we will assume that there exists no $(a, b) \in M_{d_{t}}^{\text {opt }}$ optimal to the unrestricted problem $\left(P_{d_{t}}\right)$ such that the slope of the corresponding line $l_{a, b}$ is equal to the slope $s_{t}$ of the vector $t$, that is $a=\frac{t_{2}}{t_{1}}$. This is equivalent to assuming 
that no vertical line is optimal for the corresponding vertical problem $\tilde{M}_{v e r}^{\text {opt }}$ in the transformed system which is in line with the assumption that no vertical line is optimal to $\left(P_{v e r}\right)$ we made in Chapter $\llbracket$ as well as to the one that no horizontal line is optimal to $\left(P_{h o r}\right)$ we made in Chapter III. As it was the case in the earlier chapters, this is again no restriction.

This is all we need to prove the following theorem.

Theorem VI.1.1. For all distances $d_{t}$ the following criteria hold.

1. For all $s \geq 0$ there exists some $(a, b) \in M_{d_{t}}^{\text {opt }}(s)$ optimal for $\left(P_{d_{t}}(s)\right)$ such that the corresponding line $l_{a, b}$ passes through at least one of the existing facilities.

2. All lines optimal for $\left(P_{d_{t}}(s)\right)$ are pseudo-halving.

3. Let $s^{*}=\min \left\{|a|:(a, b) \in M_{d_{t}}^{\text {opt }}\right\}$. For all $s \in\left[0, s^{*}\right]$ it holds that $|a|=s$ if $(a, b) \in M_{d_{t}}^{o p t}(s)$.

Proof. Keep in mind that we can reformulate $\left(P_{d_{t}}(s)\right)$ as

$$
\begin{aligned}
& \min \sum_{i=1}^{n} d_{v e r}\left(\left(\tilde{x}_{i}, \tilde{y}_{i}\right), l_{\tilde{a}, \tilde{b}}\right) \\
& \text { s.t. }(\tilde{a}, \tilde{b}) \in \tilde{M}_{v e r}
\end{aligned}
$$

with $\tilde{M}_{v e r} \in\left\{\tilde{M}_{v e r}^{1}, \tilde{M}_{v e r}^{2}\right\}$.

1. Let us look at the dual space of the vertical problem in the transformed system. Our existing facilities again imply a cell structure and since we are looking at a problem with vertical distance we already know that our objective function is linear on each cell.

Let us first assume that $\tilde{M}_{v e r}=\tilde{M}_{v e r}^{1}$. As in the vertical case the restriction on the slope gives us two additional vertical lines in the dual space passing the $\tilde{a}$-axis at $\tilde{s}_{1}$ and $\tilde{s}_{2}$, respectively. Therefore it exists an optimal solution $(\tilde{a}, \tilde{b})$ for $\left(\tilde{P}_{v e r}(\tilde{s})\right)$ that corresponds to a vertex of a cell in the dual space following the same argumentation as in the proof of Theorem I.2.1. But if $(\tilde{a}, \tilde{b})$ is a vertex of a cell in the dual space that means that the corresponding line passes through at least one of the existing facilities. This means that 
$D^{-1}\left(l_{\tilde{a}, \tilde{b}}\right)$ is a line optimal for $\left(P_{d_{t}}(s)\right)$ that passes through at least one of the existing facilities since $D$ is a linear transformation and therefore

$$
(x, y) \in l_{a, b} \Leftrightarrow D(x, y) \in D\left(l_{a, b}\right)
$$

Now we assume that $\tilde{M}_{v e r}=\tilde{M}_{v e r}^{2}$. As a matter of fact, it can be shown that there exists a line $l_{\tilde{a}, \tilde{b}}$ optimal to $\left(\tilde{P}_{\text {ver }}(\tilde{s})\right)$ such that the corresponding point $(\tilde{a}, \tilde{b})$ in the dual space is a vertex of a cell implied by the lines corresponding to the existing facilities and the two vertical lines given through the restriction on the slope in analogy to Theorem III.1.2. Again $D^{-1}\left(l_{\tilde{a}, \tilde{b}}\right)$ gives us a line optimal to $\left(P_{d_{t}}(s)\right)$ passing through at least one of the given facilities.

2. It is shown in [Sch99], Theorem 3.1, that the pseudo-halving property is not affected by the transformation $D$. Showing that any optimal solution to $\left(\tilde{P}_{v e r}(\tilde{s})\right)$ is pseudo-halving can be done analogously to the proof of Theorem I.2.2.

3. Let $s \in\left[0, s^{*}\right]$. To prove the third statement we can show that for all optimal solutions $(\tilde{a}, \tilde{b})$ of $\left(\tilde{P}_{v e r}(\tilde{s})\right)$ it has to hold that either $\tilde{a}=\tilde{s}_{1}$ or $\tilde{a}=\tilde{s}_{2}$. This can be done analogously to the proof of Theorem I.2.5 if $\tilde{M}_{v e r}=\tilde{M}_{v e r}^{1}$ or analogously to the proof of Theorem III.1.4 if $\tilde{M}_{v e r}=\tilde{M}_{v e r}^{2}$. But this means exactly that $(a, b)=D^{-1}(\tilde{a}, \tilde{b})$ satisfies either $a=-s$ or $a=s$, in other words it is indeed $|a|=s$.

Remark VI.1.1. Note that for $t$-distances we cannot state a result that is a direct analogon to Theorem 1.2.4, in other words it does not need to hold that all lines optimal to the restricted problem $\left(P_{d_{t}}(s)\right)$ share the same sign in slope. We cannot do better than we did in the third statement of TheoremVI.1.1. The reason is the same as it was in Chapter III for $\left(P_{\text {hor }}(s)\right)$ : The corresponding problem $\left(\tilde{P}_{\text {ver }}(\tilde{s})\right)$ does not have to be convex any more. In fact, it is not convex if $\tilde{M}_{v e r}=\tilde{M}_{v e r}^{2}$. It is not hard to see that this is the case if the slope of the vector $t$ is less than $s$, that is $\frac{t_{1}}{t_{1}}<s$. 
We already mentioned that the horizontal distance is a special case of the $t$-distance, and therefore it is an example for a problem with $t$-distance having a corresponding problem $\left(\tilde{P}_{v e r}(\tilde{s})\right)$ that is not convex. As a consequence, there need not exist a continuous optimal trajectory to our line location problem with t-distance $\left(P_{d_{t}}(s)\right)$.

Up to now we have shown that most of the results derived in Chapter I and in Chapter III hold again if we consider $t$-distances. The original aim of this section was to carry the results of these chapters over to distances derived from a norm. Fortunately [Sch99] provides the connection between any norm $\gamma$ with corresponding distance $d$ and the distances $d_{t}$.

Lemma VI.1.5. ([Sch99]) Let $\gamma$ be a norm and d the corresponding distance. Let $(x, y) \in \mathbb{R}^{2}$ and $l_{a, b}$ be a line. Then

$$
d\left((x, y), l_{a, b}\right)=\min _{t \in \mathbb{R}^{2}, \gamma(t)=1} d_{t}\left((x, y), l_{a, b}\right)
$$

Lemma VI.1.6. ([Sch99]) Let $\gamma$ be a norm and d the corresponding distance. Let $l_{a, b}$ be a line. Then there exists a $t \in \mathbb{R}^{2}$ with $\gamma(t)=1$ such that

$$
d\left((x, y), l_{a, b}\right)=d_{t}\left((x, y), l_{a, b}\right) \text { for all }(x, y) \in \mathbb{R}^{2} .
$$

We can now prove the following theorem.

Theorem VI.1.2. For all distances d derived from norms the following criteria hold.

1. For all $s \geq 0$ there exists some $(a, b) \in M_{d}^{\text {opt }}(s)$ optimal for $\left(P_{d}(s)\right)$ such that the corresponding line $l_{a, b}$ passes through at least one of the existing facilities.

2. All lines optimal for $\left(P_{d}(s)\right)$ are pseudo-halving.

3. Let $s^{*}=\min \left\{|a|:(a, b) \in M_{d}^{\text {opt }}\right\}$. For all $s \in\left[0, s^{*}\right]$ it holds that $|a|=s$ if $(a, b) \in M_{d}^{\text {opt }}(s)$.

Proof. 1. Suppose $l_{a^{*}, b^{*}}$ is an optimal line that does not pass through one of the existing facilities. Choose $t^{*}$ such that $d\left(\left(x_{i}, y_{i}\right), l_{a^{*}, b^{*}}\right)=d_{t^{*}}\left(\left(x_{i}, y_{i}\right), l_{a^{*}, b^{*}}\right)$ for all $\left(x_{i}, y_{i}\right) \in \mathcal{E}$ according to Lemma VI.1.6. 
From Theorem VI.1.1 we know that there exists a line $l_{\hat{a}, \hat{b}}$ optimal to $\left(P_{t_{t^{*}}}(s)\right)$ that passes through at least one of the existing facilities.

Now let $\hat{t}$ be such that $d\left(\left(x_{i}, y_{i}\right), l_{\hat{a}, \hat{b}}\right)=d_{\hat{t}}\left(\left(x_{i}, y_{i}\right), l_{\hat{a}, \hat{b}}\right)$ for all $\left(x_{i}, y_{i}\right) \in \mathcal{E}$ according to Lemma VI.1.6. Then we get:

$$
\begin{aligned}
f_{d}\left(a^{*}, b^{*}\right) & =\sum_{i=1}^{n} d\left(\left(x_{i}, y_{i}\right), l_{a^{*}, b^{*}}\right) \\
& =\sum_{i=1}^{n} d_{t^{*}}\left(\left(x_{i}, y_{i}\right), l_{a^{*}, b^{*}}\right) \\
& \geq \sum_{i=1}^{n} d_{t^{*}}\left(\left(x_{i}, y_{i}\right), l_{\hat{a}, \hat{b}}\right) \\
& \geq \sum_{i=1}^{n} d_{\hat{t}}\left(\left(x_{i}, y_{i}\right), l_{\hat{a}, \hat{b}}\right) \text { due to Lemma VI.1.5 } \\
& =\sum_{i=1}^{n} d\left(\left(x_{i}, y_{i}\right), l_{\hat{a}, \hat{b}}\right) \\
& =f_{d}(\hat{a}, \hat{b}) \geq f_{d}\left(a^{*}, b^{*}\right) \text { due to the optimality of } l_{a^{*}, b^{*}}
\end{aligned}
$$

Therefore $l_{\hat{a}, \hat{b}}$ is an optimal line that passes through one of the existing facilities.

2. Assume that there exists a line $l_{a^{*}, b^{*}}$ optimal to $\left(P_{d}(s)\right)$ that is not pseudohalving, that is without loss of generality $\left|L\left(a^{*}, b^{*}\right)\right|>\frac{n}{2}$. With the same notation as in the first part of this proof we can conclude from Theorem VI.1.1 that any line $l_{\hat{a}, \hat{b}}$ optimal to $\left(P_{d_{t^{*}}}(s)\right)$ satisfies

$$
|L(\hat{a}, \hat{b})| \leq \frac{n}{2} \text { and }|R(\hat{a}, \hat{b})| \leq \frac{n}{2} .
$$

Since $l_{a^{*}, b^{*}}$ cannot be optimal for $\left(P_{d_{t^{*}}}(s)\right)$ according to Theorem VI.1.1, we get

$$
\begin{aligned}
f_{d}\left(a^{*}, b^{*}\right) & =\sum_{i=1}^{n} d_{t^{*}}\left(\left(x_{i}, y_{i}\right), l_{a^{*}, b^{*}}\right) \\
& >\sum_{i=1}^{n} d_{t^{*}}\left(\left(x_{i}, y_{i}\right), l_{\hat{a}, \hat{b}}\right) \\
& \geq f_{d}(\hat{a}, \hat{b})
\end{aligned}
$$


VI.2. LOCATING HYPERPLANES WITH A RESTRICTION ON RECTANGULAR DISTANCE - AN OUTLOOK

contradicting the optimality of $l_{a^{*}, b^{*}}$.

3. Assume that there exists a line $l_{a^{*}, b^{*}}$ optimal for $\left(P_{d}(s)\right)$ with $s \in\left[0, s^{*}\right]$ such that $|a|<s$. Again we want to use the same notation as in the first part of this proof. From Theorem VI.1.1 we can conclude that any line $l_{\hat{a}, \hat{b}}$ optimal for $\left(P_{d_{t^{*}}}(s)\right)$ satisfies $|\hat{a}|=s$.

Since $l_{a^{*}, b^{*}}$ cannot be optimal for $\left(P_{d_{t^{*}}}(s)\right)$ according to Theorem VI.1.1, we get

$$
f_{d}\left(a^{*}, b^{*}\right)>f_{d}(\hat{a}, \hat{b})
$$

using the same argumentation as we did in the second part of this proof. This again contradicts the optimality of $l_{a^{*}, b^{*}}$.

TheoremVI.1.2 tells us that most of the important results presented for the vertical distance in Chapter I and the horizontal distance in Chapter III can be carried over to problems with a distance derived from a norm. Note that the third statement in Theorem VI.1.2 allows that there does not exist a continuous optimal trajectory for $\left(P_{d}(s)\right)$, the trajectory might jump from one side of the $a$-axis to the other one and back as it already was the case when considering problems using the horizontal distance in Chapter III or problems using the $t$-distance earlier in this section.

\section{VI.2 Locating hyperplanes with a restriction on the slope considering horizontal and rect- angular distance - an outlook}

In this section we want to discuss the possibility of carrying over results derived in Chapter $\mathrm{V}$ for hyperplane location with vertical distance to the case of locating hyperplanes with a restriction on the slope first with horizontal distance and then with rectangular distance. Remember that in the case of line location we were able to show that most of the results derived for line location with vertical distance in Chapter \ hold as well for the problem with horizontal distance (see Chapter III) 
and the one with rectangular distance (see Chapter IV). The major difference between the problem with vertical distance on the one hand and the one with horizontal distance as well as the one with rectangular distance on the other hand was that the continuity of the optimal trajectory was lost.

Even if we do not want to discuss in detail which of the results in Chapter $\mathrm{V}$ can be carried over to other distances, we want to give an idea of why we think that there is a very good chance that most of the results concerning the vertical distance will hold again when considering problems with horizontal distance and rectangular distance, respectively.

Let us start with discussing the problem of locating a hyperplane with a restriction on the slope using the horizontal distance. Let $\mathcal{E}=\left\{\left(x_{1}, y_{1}\right), \ldots,\left(x_{n}, y_{n}\right)\right\} \subseteq \mathbb{R}^{k+1}$ again be the set of existing facilities. Assume that no horizontal hyperplane as well as no vertical hyperplane is optimal to the unrestricted problem of locating a hyperplane that minimizes the sum of distances between the existing facilities and the new hyperplane. This assumption is in line with the assumption we made in Chapter III that no horizontal line as well as no vertical line is optimal to $\left(P_{h o r}\right)$. Again, it is no restriction to assume this.

The problem that we want to solve is the following optimization problem

$$
\begin{aligned}
& \min f_{h o r}(a, b)=\min \sum_{i=1}^{n} d_{h o r}\left(\left(x_{i}, y_{i}\right), H_{a, b}\right) \quad\left(P_{h o r}(s)\right) \\
& \text { s.t. }\|a\|_{1} \leq s
\end{aligned}
$$

with $s \geq 0$. The horizontal distance between a point $(x, y) \in \mathbb{R}^{k+1}$ and a hyperplane $H$ is defined as

$$
d_{\text {hor }}((x, y), H)=\min _{\left(x^{\prime}, y^{\prime}\right) \in H} d_{h o r}\left((x, y),\left(x^{\prime}, y^{\prime}\right)\right)
$$

For the horizontal distance between a point $(x, y)$ and a non-vertical, nonhorizontal hyperplane $H_{a, b}$ the following formula is known (see e.g. [Sch99]): 


\section{VI.2. LOCATING HYPERPLANES WITH A RESTRICTION ON THE SLOPE CONSIDERING HORIZONTAL AND RECTANGULAR DISTANCE - AN OUTLOOK}

$$
\begin{aligned}
d_{h o r}\left((x, y), H_{a, b}\right) & =\mid \frac{1}{a_{1}}\left(a_{1} x_{1}-\left(y-\sum_{j=2}^{k} a_{j}-b\right) \mid\right. \\
& =\left|\frac{1}{a_{1}}\right| d_{v e r}\left((x, y), H_{a, b}\right) .
\end{aligned}
$$

When we say that we consider it as very likely that most of the results derived in Chapter $\mathrm{V}$ can be carried over to $\left(P_{\text {hor }}(s)\right)$, what we have in mind is that the following statements are very likely to be true:

1. For all $s \geq 0$ there exists some $(a, b) \in M_{d_{h o r}}^{\text {opt }}(s)$ optimal for $\left(P_{d_{\text {hor }}}(s)\right)$ such that the corresponding hyperplane $H_{a, b}$ passes through at least one of the existing facilities.

2. All hyperplanes optimal for $\left(P_{d_{h o r}}(s)\right)$ are pseudo-halving.

3. Let $s^{*}=\min \left\{\|a\|_{1}:(a, b) \in M_{d_{h o r}}^{\text {opt }}\right\}$. For all $s \in\left[0, s^{*}\right]$ it holds that $\|a\|_{1}=s$ if $(a, b) \in M_{d_{\text {hor }}}^{\text {opt }}(s)$.

The reason why we assume that these results hold in the horizontal case is that we think the problem $\left(P_{h o r}(s)\right)$ is equivalent to a problem $\left(\tilde{P}_{v e r}(\tilde{s})\right)$ with vertical distance as it is the case when locating a line. What we need to prove this is to show that there exists a transformation $T: \mathbb{R}^{k+1} \rightarrow \mathbb{R}^{k+1}$ that satisfies two conditions: First, $T$ has to satisfy $d_{h o r}\left((x, y), H_{a, b}\right)=d_{v e r}\left(T(x, y), T\left(H_{a, b}\right)\right)$ for all $(x, y) \in \mathbb{R}^{k+1}$ and all non-vertical, non-horizontal hyperplanes $H_{a, b}$. Second, the transformed set of feasible solutions, that is $\tilde{M}_{v e r}(\tilde{s})=\left\{(\tilde{a}, \tilde{b}): H_{\tilde{a}, \tilde{b}}=\right.$ $T\left(H_{a, b}\right)$ s.t. $\left.(a, b) \in M_{h o r}(s)\right\}$, must be connected to the set of feasible solutions $M_{\text {hor }}(s)=\left\{(a, b):\|a\|_{1} \leq s\right\}$ of $\left(P_{h o r}(s)\right)$ in a nice way. "In a nice way" means that a hyperplane $H_{\tilde{a}, \tilde{b}}$ corresponding to a point $(\tilde{a}, \tilde{b})$ lying on the boundary of the feasible region in the dual space of the problem $\left(\tilde{P}_{v e r}(\tilde{s})\right)$ should lead to a hyperplane $H_{a, b}=T^{-1}\left(H_{\tilde{a}, \tilde{b}}\right)$ corresponding to a point $(a, b)$ lying on the boundary of the feasible region in the dual space of $\left(P_{h o r}(s)\right)$. Furthermore the feasible region in the dual space of $\left(\tilde{P}_{v e r}(\tilde{s})\right)$ should be bounded by $2^{k}$ hyperplanes in some way as it is the case in the dual space of $\left(P_{\text {hor }}(s)\right)$. 
It is easy to find a transformation $T$ that satisfies the first condition. Take for example the transformation $T$ that maps each point $\left(x_{1}, \ldots, x_{k}, y\right)$ to the point $\left(-y, x_{2}, x_{3}, \ldots, x_{k}, x_{1}\right)$ and each hyperplane $H_{a, b}$ to the hyperplane

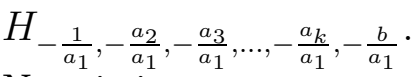

Now it is

$$
\begin{aligned}
d_{v e r}\left(T(x, y), T\left(H_{a, b}\right)\right) & =d_{v e r}\left(\left(-y, x_{2}, x_{3}, \ldots, x_{k}, x_{1}\right), H_{\left.-\frac{1}{a_{1}},-\frac{a_{2}}{a_{1}},-\frac{a_{3}}{a_{1}}, \ldots,-\frac{a_{k}}{a_{1}},-\frac{b}{a_{1}}\right)}\right. \\
& =\left|x_{1}-\left(\frac{y}{a_{1}}-\sum_{j=2}^{k} \frac{a_{j}}{a_{1}}-\frac{b}{a_{1}}\right)\right| \\
& =\mid \frac{1}{a_{1}}\left(a_{1} x_{1}-\left(y-\sum_{j=2}^{k} a_{j}-b\right) \mid\right. \\
& =d_{h o r}\left((x, y), H_{a, b}\right) .
\end{aligned}
$$

Unfortunately it is not that easy to see what happens to the set of feasible solutions under this transformation. Anyway, even if we do not know yet which transformation to choose we still think that there exists a transformation such that $\left(P_{\text {hor }}(s)\right)$ is equivalent to a vertical problem $\left(\tilde{P}_{v e r}(\tilde{s})\right)$ while the set of feasible solutions has the properties we need to carry over the results 1.-3. from the vertical case to the horizontal case. Note that the transformed set of feasible solutions $\tilde{M}_{v e r}(\tilde{s})$ needs not to be convex any more, therefore there need not exist a continuous optimal trajectory for $\left(P_{\text {hor }}(s)\right)$.

Assume that it is possible to prove statements 1.-3. for the horizontal case by showing the equivalence between $\left(P_{\text {hor }}(s)\right)$ and a problem $\left(\tilde{P}_{\text {ver }}(\tilde{s})\right)$ with vertical distance. Note that the horizontal distance $d_{h o r}$ gives the distance between two points in the $\mathbb{R}^{k+1}$ in the $e_{1}$-direction while the vertical distance $d_{v e r}$ gives the distance between two points in the $\mathbb{R}^{k+1}$ in the $e_{k+1}$-direction, where $e_{1}$ and $e_{k+1}$ are the first and the $(k+1)$-th unit vector of $\mathbb{R}^{k+1}$. Let us define the distances in the other directions $e_{2}, \ldots, e_{k}$ as 


\section{VI.2. LOCATING HYPERPLANES WITH A RESTRICTION ON

$$
\begin{aligned}
d_{e_{\hat{j}}}\left((x, y), H_{a, b}\right) & =\mid \frac{1}{a_{\hat{j}}}\left(a_{\hat{j}} x_{\hat{j}}-\left(y-\sum_{j \in\{1, \ldots, k\} \backslash\{\hat{j}\}} a_{j}-b\right) \mid\right. \\
& =\left|\frac{1}{a_{\hat{j}}}\right| d_{v e r}\left((x, y), H_{a, b}\right) \text { for } \hat{j} \in\{2, \ldots, k\}
\end{aligned}
$$

If we assume that for the unrestricted problem $\left(P_{d_{e_{j}}}\right), j=2, \ldots, k$, there exists no optimal hyperplane that is parallel to one of the $x_{j}$-axes or the $y$-axis we obtain that statements 1.-3. hold for $\left(P_{d_{e_{j}}}(s)\right)$ as well analogous to the horizontal problem $\left(P_{\text {hor }}(s)\right)$.

Finally, the rectangular distance between a point $(x, y) \in \mathbb{R}^{k+1}$ and a hyperplane $H_{a, b} \subseteq \mathbb{R}^{k+1}$ is given by

$$
l_{1}\left((x, y), H_{a, b}\right)=\min _{j=1, \ldots, k+1} d_{e_{j}}\left((x, y), H_{a, b}\right) .
$$

Therefore statements 1.-3. also hold for $\left(P_{l_{1}}(s)\right)$.

Summarizing this section, we presented our argument for the assumption that most of the results derived in Chapter $\square$ for $\left(P_{\text {ver }}(s)\right)$ can be carried over to problems considering the horizontal distance or distances in the direction of unit vectors in general. This would directly lead to the statement that most of the results derived for $\left(P_{v e r}(s)\right)$ hold for the problem of locating a hyperplane with a restriction on the slope using the rectangular distance as well since the rectangular distance between a point and a hyperplane is the minimum of all distances in $e_{j}$-direction, $j=1, \ldots, k+1$, between this point and the hyperplane.

It is needless to say that the assumptions we expressed in this section have to be proven in detail before it is certain that we can indeed carry over our results of Chapter $\nabla$ to other distances. Especially the existence of a problem $\left(\tilde{P}_{v e r}(\tilde{s})\right)$ with vertical distance that is equivalent to the horizontal problem $\left(P_{\text {hor }}(s)\right)$ has to be ascertained. These tasks are left to future work. 


\section{Bibliography}

[AF90] Jean-Pierre Aubin and Hélène Frankowska. Set-valued analysis. Birkhäuser Boston, Inc., Boston, MA, 1990.

[BC91] Margaret L. Brandeau and Samuel S. Chiu. Parametric analysis of optimal facility locations. NETWORKS, 21:223-243, 1991.

[BCH09] Rafael Blanquero, Emilio Carrizosa, and Pierre Hansen. Locating objects in the plane using global optimization techniques. Mathematics of Operations Research, 34(4):837-858, 2009.

[BJS09] J. Brimberg, H. Juel, and A. Schöbel. Locating a minisum circle in the plain. Discrete applied mathematics, 157:901-912, 2009.

[BR73] I. Barrodale and F.D.K. Roberts. An improved algorithm for discrete 11 linear approximation. SIAM Journal on Numerical Analysis, 10:839848, 1973.

[DBMS04] J.M. Díaz-Báñez, J.A. Mesa, and A. Schöbel. Continuous location of dimensional structures. European Journal of Operational Research, 152(1):22-44, 2004.

[DH02] Zvi Drezner and Horst W. Hamacher, editors. Facility Location. Applications and theory. Springer-Verlag Berlin, 2002.

[Edg88] F.Y. Edgeworth. On a new method of reducing observations relating to several quantities. Philosophical Magazine (Series 5), 25:184-191, 1888. 
[EHJT04] Bradley Efron, Trevor Hastie, Iain Johnstone, and Robert Tibshirani. Least angle regression. with discussion, and a rejoinder by the authors. Ann. Statist., 32(2):407-499, 2004.

[Ehr05] Matthias Ehrgott. Multicriteria Optimization. Springer-Verlag, Berlin, second edition, 2005.

[GP02] A. Giloni and M. Padberg. Alternative methods of linear regression. Mathematical and Computer Modelling, 35:361-374, 2002.

[GP04] A. Giloni and M. Padberg. The finite sample breakdown point of 11regression. SIAM Journal on Optimization, 14:1028-1042, 2004.

[Ham95] Horst W. Hamacher. Mathematische Lösungsverfahren für planare Standortprobleme. Vieweg, 1995.

[Hay81] W.L. Hays. Statistics. Holt, Rinehart and Winston, 3 edition, 1981.

[HJKP90] X. He, J. Jurechova, R. Koenker, and S. Portnoy. Tail behavior of regression estimators and their breakdown points. Econometrica, 58:1195-1214, 1990.

[HLW71] Y.Y. Haimes, L.S. Lasdon, and D.A. Wismer. On a bicriterion formulation of the problems of integrated system identification and system optimization. IEEE Transactions on Systems, Man, and Cybernetics, 1:296-297, 1971.

[HSL93] S.L. Hakimi, E.F. Schmeichel, and Martine Labbe. On locating pathor tree-shaped facilities on networks. Networks, 23:543-555, 1993.

[Jun08] Dieter Jungnickel. Optimierungsmethoden: Eine Einführung. SpringerLehrbuch. Springer-Verlag Berlin, 2008.

[Kel55] John L. Kelley. General Topology. D. Van Nostrand Company, Inc., Toronto-New York-London, 1955. 
[KM93] N.M. Korneenko and H. Martini. Hyperplane approximation and related topics. In J. Pach, editor, New Trends in Discrete and Computational Geometry, chapter 6, pages 135 - 162. Springer-Verlag, New York, Inc., 1993.

[Kör11] Mark-Christoph Körner. Minisum Hyperspheres. Number 51 in Springer Optimization and Its Applications. Dordrecht: Springer, 2011.

[Kre91] U. Krengel. Einführung in die Wahrscheinlichkeitstheorie und Statistik, volume 59 of Vieweg Studium. Vieweg, Braunschweig, 1991.

[LMW88] R.F. Love, J.G. Morris, and G.O. Wesolowsky. Facilities location. North-Holland, Amsterdam, 1988.

[MB96] J.A. Mesa and T.B. Boffey. A review of extensive facility location in networks. European Journal of Operational Research, 95(3):592-603, 1996.

[Mic56] Ernest Michael. Continuous selections. i. Ann. of Math. (2), (63):361$382,1956$.

[Min67] Hermann Minkowski. Gesammelte Abhandlungen, volume Band 2. Chelsea Publishing Company, New York, 1967.

[MM01] I. Mizera and C.H. Müller. The influence of the design on the breakdown points of 11-type m-estimators. In mODa 6 - Advances in modeloriented design and analysis. Proceedings of the 6th international workshop, Puchberg/ Schneeberg, Austria, June 25-29, 2001., pages 193200. Physica-Verlag, Heidelberg, 2001.

[MN80] J.G. Morris and J.P. Norback. A simple approach to linear facility location. Transportation Science, 14(1):1-8, 1980.

[MN83] J.G. Morris and J.P. Norback. Linear facility location - solving extensions of the basic problem. European Journal of Operational Research, 12:90-94, 1983. 
[MT83] N. Megiddo and A. Tamir. Finding least-distance lines. SIAM Journal on Algebraic and Discrete methods, 4(2):207-211, 1983.

[NW88] G.L. Nemhauser and L.A. Wolsey. Integer and Combinatorial Optimization. John Wiley \& Sons, New York-Chichester-Brisbane-TorontoSingapore, 1988.

[PC01] F. Plastria and E. Carrizosa. Gauge distances and median hyperplanes. Journal of Optimization Theory and Applications, 110(1):173-182, July 2001 .

[PFTV86] W.H. Press, B.P. Flannery, S.A. Teulosky, and W.T. Vetterling. Numerical Recipes. Cambridge University Press, Cambridge, New York, 1986.

[Pla95] Frank Plastria. Facility Location: A survey of applications and methods. Springer-Verlag, New York, Inc., 1995.

[Ric64] J. Rice. The approximation of functions, volume 1. Addison-Wesley, 1964.

[RZ07] Saharon Rosset and Ji Zhu. Piecewise linear regularized solution paths. The Annals of statistics, 35(3):1012-1030, 2007.

[Sch99] Anita Schöbel. Locating Lines and Hyperplanes. Kluwer Academic Press, 1999.

[Sha71] W.F. Sharpe. Mean-absolute-deviation characteristic lines for securities and portfolios. Management Science, 18:B1-B13, 1971.

[Sha78] M.I. Shamos. Computational Geometry. PhD thesis, Department of Computer Science, Yale University, New Haven, 1978.

[Sto48] A.H. Stone. Paracompactness and product spaces. Bull. Amer. Math. Soc., 54:977-982, 1948.

[SW87] H. Späth and G.A. Watson. On orthogonal linear 11 approximation. Numerische Mathematik, 51:531-543, 1987. 
[Tib96] Robert Tibshirani. Regression shrinkage and selection via the lasso. $J$. Royal. Statist. Soc B., 58(1):267 - 288, 1996.

[WGZ06] Li Wang, Michael D. Gordon, and Ji Zhu. Regularized least absolute deviations regression and an efficient algorithm for parameter tuning. Proceedings of the Sixth Internetional Conference on Data Mining (ICDM'06), pages $690-700,2006$. 